Supplement of Geosci. Model Dev., 9, 1747-1802, 2016

http://www.geosci-model-dev.net/9/1747/2016/

doi:10.5194/gmd-9-1747-2016-supplement

(C) Author(s) 2016. CC Attribution 3.0 License.

(c) (i)

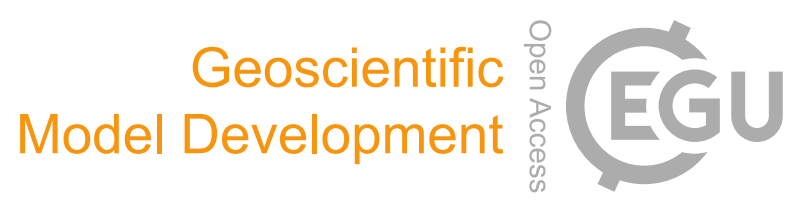

Supplement of

\title{
ESMValTool (v1.0) - a community diagnostic and performance metrics tool for routine evaluation of Earth system models in CMIP
}

Veronika Eyring et al.

Correspondence to: Veronika Eyring (veronika.eyring@dlr.de)

The copyright of individual parts of the supplement might differ from the CC-BY 3.0 licence. 


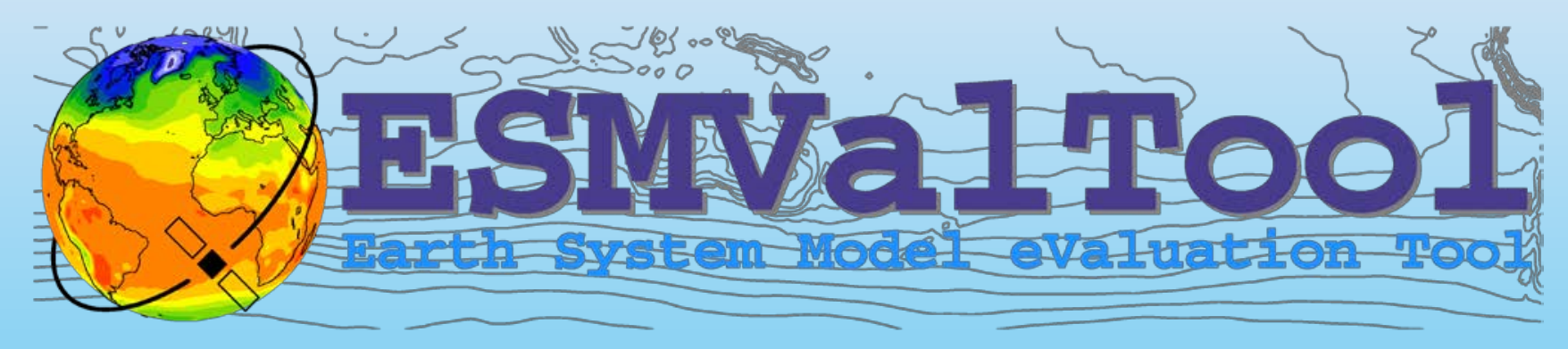

\section{User's and Developer's Guide}

May 2016

Deutsches Zentrum für Luft- und Raumfahrt (DLR), Institut für Physik der Atmosphäre, Oberpfaffenhofen, Germany http://www.esmvaltool.org/ 


\section{ESMValTool v1.0 User's and Developer's Guide}

\section{Contents}

Preface

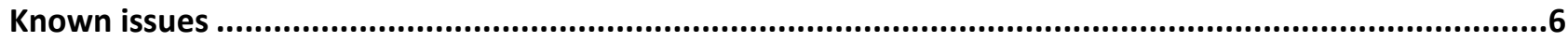

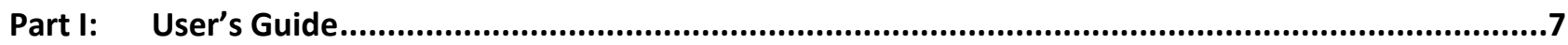

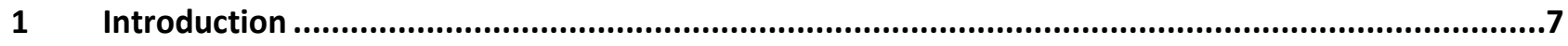

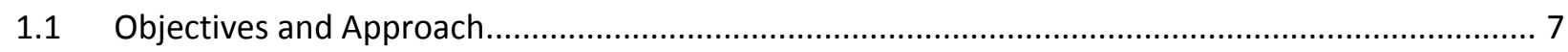

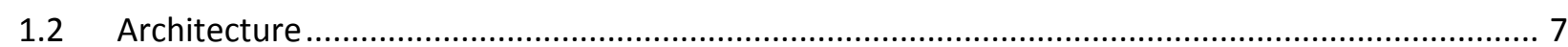

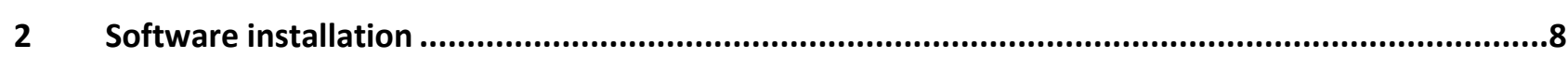

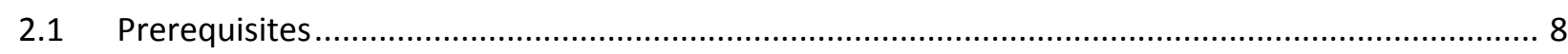

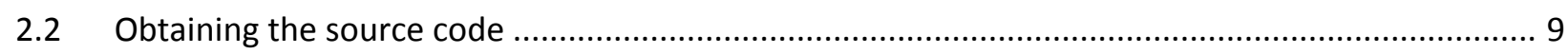

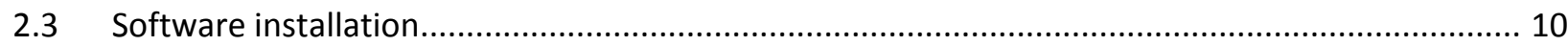

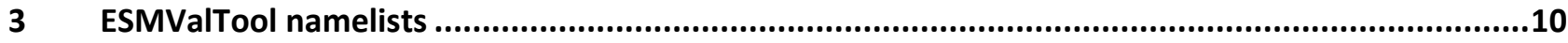

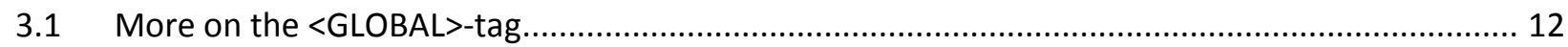

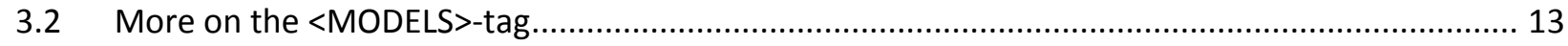

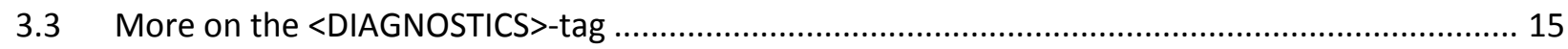

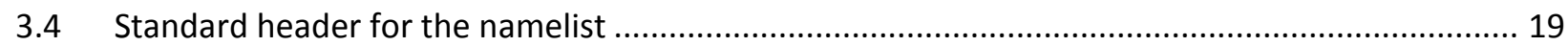

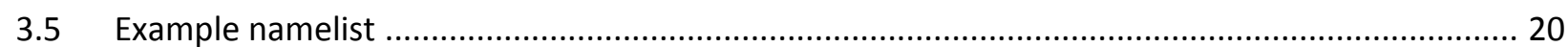

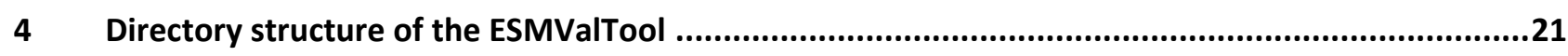

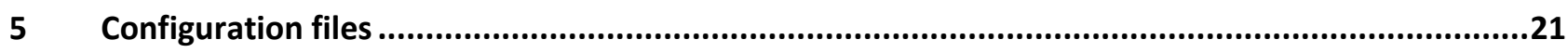

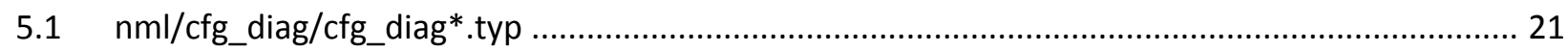

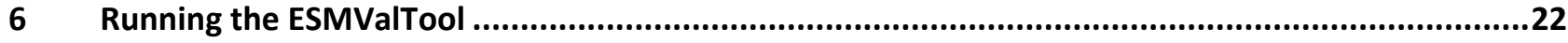

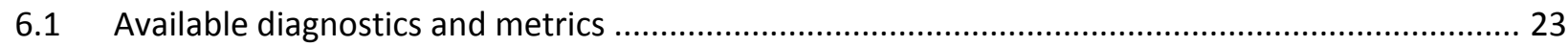

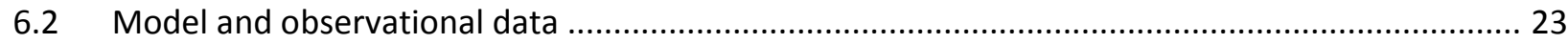

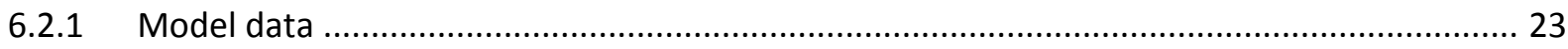

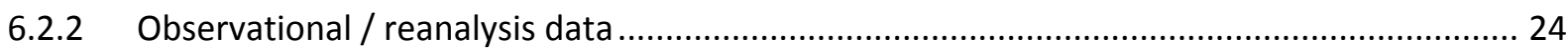

6.2.3 Downloading and creating observational data sets .......................................................... 25

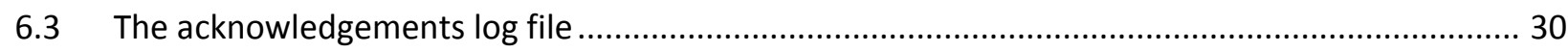

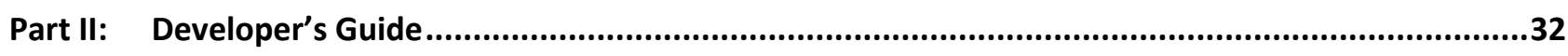

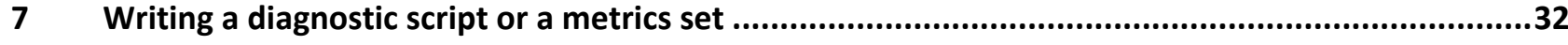

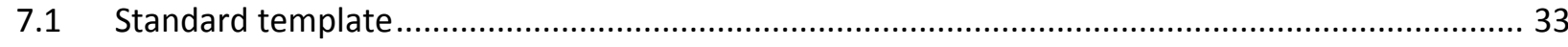




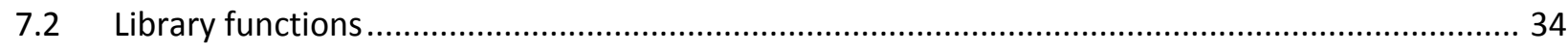

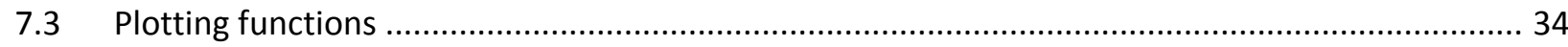

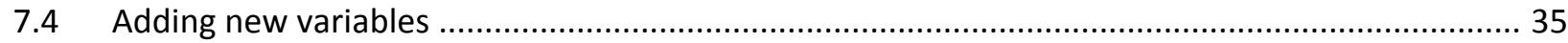

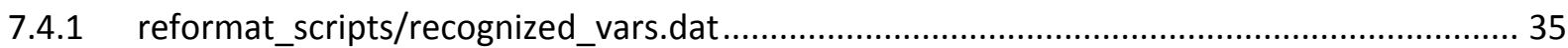

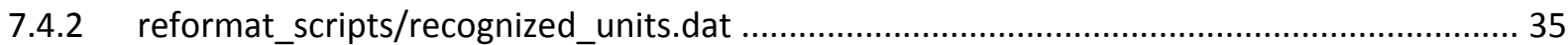

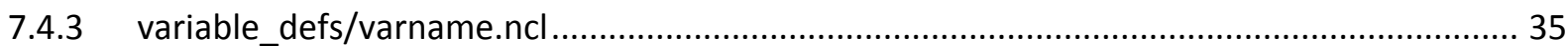

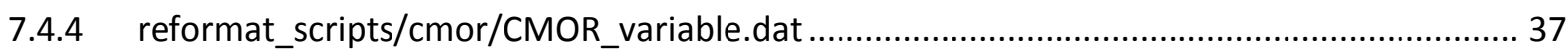

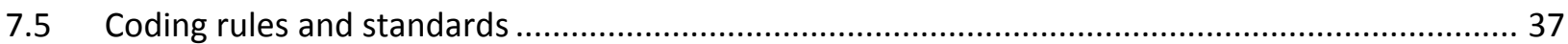

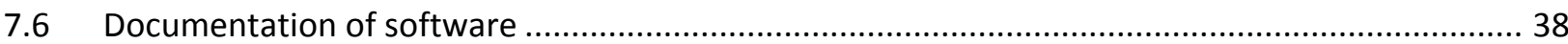

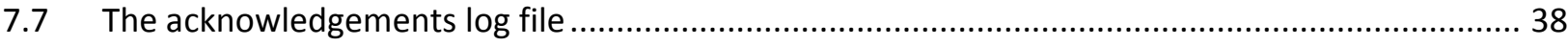

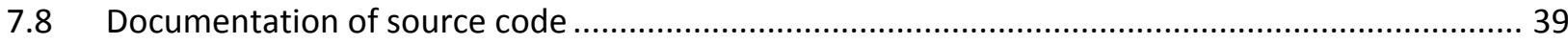

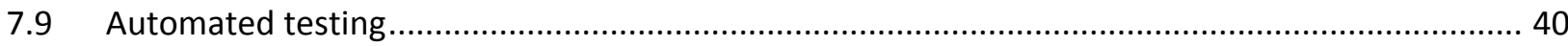

7.9.1 Setup and general workflow............................................................................ 40

7.9.2 Example test implementation for a diagnostic ......................................................... 41

8 Scientific documentation of a diagnostic script or metrics set..................................................42

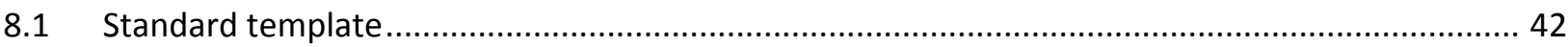

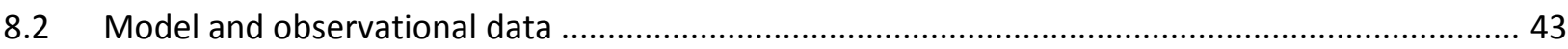

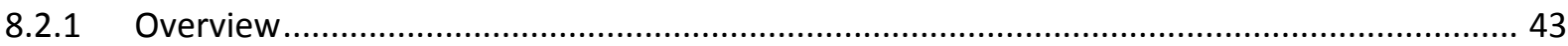

8.2.2 Standard header for the reformatting routines for observational data .........................43

9 The ESMValTool core development team .......................................................................44

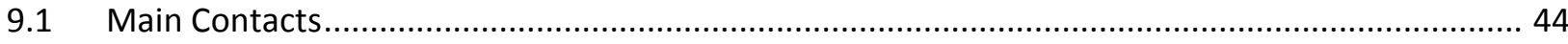

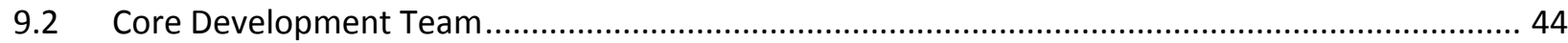

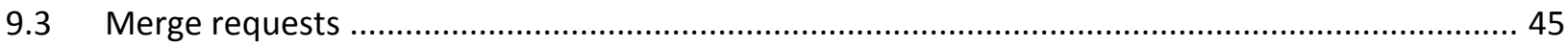

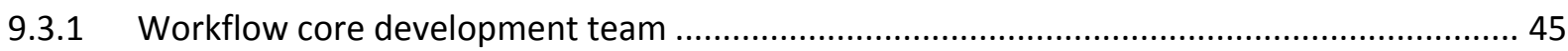

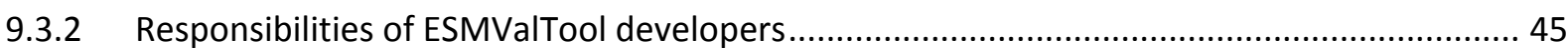

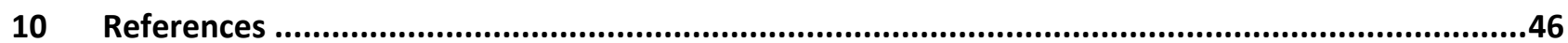

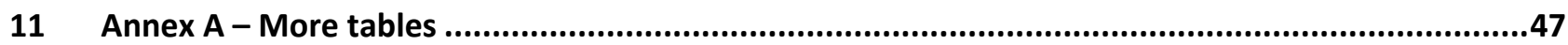

12 Annex B - subversion, Mantis, wiki ................................................................................49

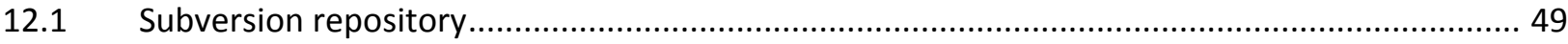

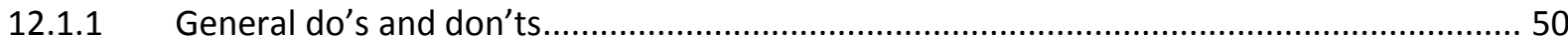

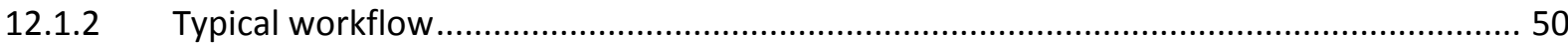

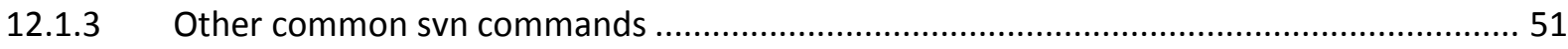

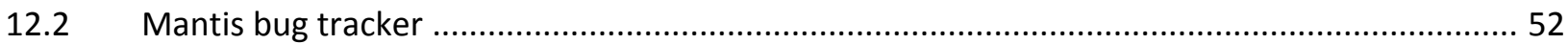

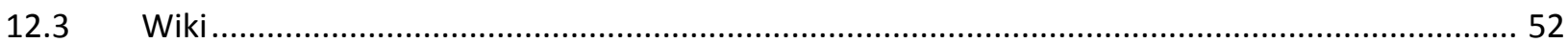

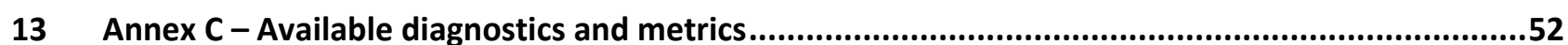


Aerosol

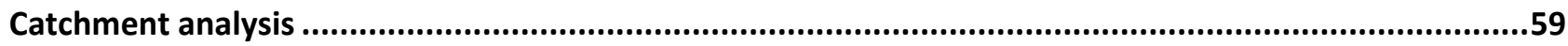

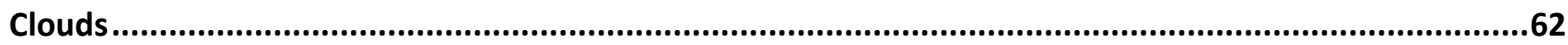

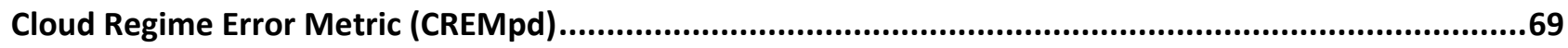

Diurnal cycle of convection ...............................................................................................71

Emergent constraints on carbon cycle feedbacks.................................................................77

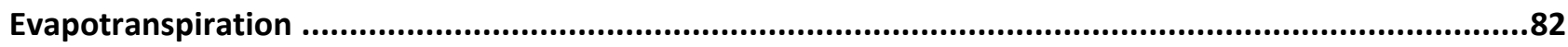

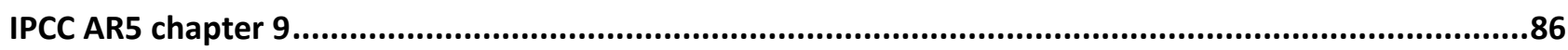

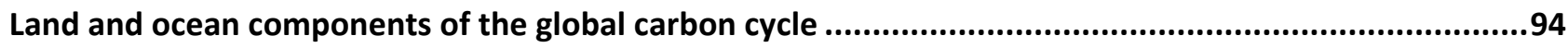

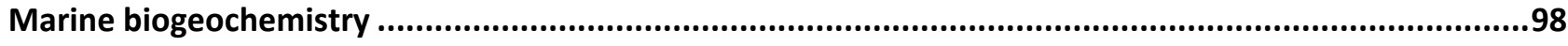

Madden-Julian Oscillation (MJO) ...................................................................................103

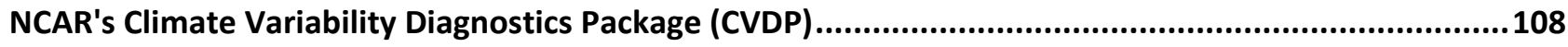

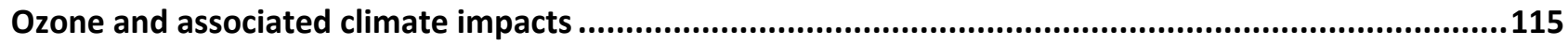

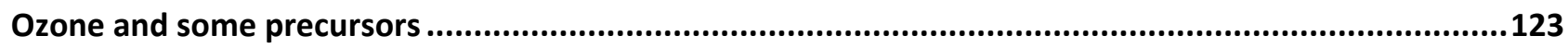

Performance metrics for essential climate parameters...................................................................130

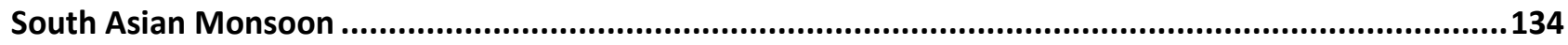

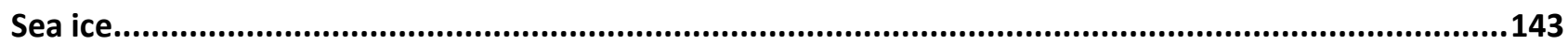

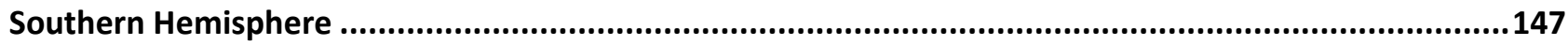

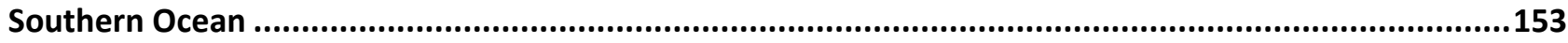

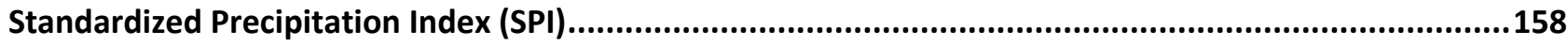

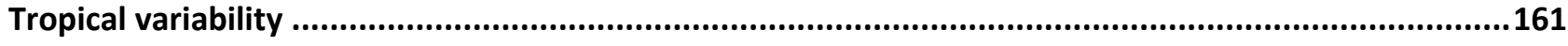

West African Monsoon...............................................................................................165 


\section{Preface}

This user's and developer's guide consists of parts targeting two overlapping categories of scientists working with the Earth System Model Evaluation Tool (ESMValTool):

(1) Part I: User's Guide: this part gives an introduction to the ESMValTool including installation, running the ESMValTool, and available user settings of existing diagnostics and performance metrics. The target group would typically consist of scientists mostly interested in running the ESMValTool as provided either on CMIP model simulations or on simulations performed with other Earth system models, and on observations. An overview on the available diagnostics and metrics packages including a description of the user settings and example plots can be found in Annex C (section 13).

(2) Part II: Developer's Guide: this part gives additional technical details on the ESMValTool not necessarily needed to apply the ESMValTool as well as an introduction to implementing new variables and new diagnostics. This part is mostly intended for scientists interested in technical details as well as in contributing to the development of the ESMValTool by adding new nameslists and code for additional diagnostics or performance metrics.

For the developer's guide (part II), it is assumed that the user/developer is already familiar with the ESMValTool framework introduced in part I.

Please report problems and bugs to the ESMValTool Core Development Team (section 9.2 and http://www.esmvaltool.org/). Thank you! 


\section{Known issues}

\section{NCL 6.2.1+}

- The function "isfilepresent" has been updated in NCL 6.2.1 in a way that is backwards-incompatible. A new function "fileexists" has been introduced with NCL v6.2.1:

https://www.ncl.ucar.edu/prev releases.shtml\#BackwardsIncompatibleChanges6.2.1

This issue can be addressed by using the ESMVal-function "isfilepresent_esmval" instead of "isfilepresent" and "fileexists".

\section{NCL 6.3.0}

- WAMonsoon: There is a problem with the routine mreg part corr (local opt should be removed and undef("mreg_part_corr") should be positioned just before.

- A missing "undef" before the declaration of the function "mreg_part_corr" in the NCL library "\$NCARG_ROOT/lib/ncarg/nclscripts/csm/shea_util.ncl" causes the ESMValTool to crash when this library is loaded more than once within the same execution. This unfortunately happens quite often within the tool, since libraries can be loaded multiple times within the various scripts (diag_scripts, lib/ncl, plot_scripts).

\section{Namelist "SouthernHemisphere"}

- Models with "dash =0" in their style definition (diag_scripts/lib/python/style.cfg) will not be plotted in the "fraction occurrence histograms of binned cloud cover" plots created by the diagnostic script diag_scripts/SoutherHemisphere_scatter.py. 


\section{Part I:User's Guide}

\section{Introduction}

The Earth System Model Evaluation Tool (ESMValTool) is a community-development that aims at improving diagnosing and understanding of the causes and effects of model biases and inter-model spread. The ESMValTool is open to both users and developers encouraging open exchange of diagnostic source code and evaluation results from the Coupled Model Intercomparison Project (CMIP) ensemble. This will facilitate and improve ESM evaluation beyond the state-of-the-art and aims at supporting the activities within CMIP and at individual modelling centers. We envisage running the ESMValTool routinely on the CMIP model output utilizing observations available through the Earth System Grid Federation (ESGF) in standard formats (obs4MIPs) or made available at ESGF nodes.

The goal is to develop a benchmarking and evaluation tool that produces well-established analyses as soon as model output from CMIP simulations becomes available, e.g., at one of the central repositories of the ESGF. This is realized through standard namelists that reproduce a certain set of diagnostics and performance metrics that have demonstrated its importance in benchmarking Earth System Models (ESMs) in a paper or assessment report, such as Chapter 9 of the Intergovernmental Panel on Climate Change (IPCC) Fifth Assessment Report (AR5) (Flato et al., 2013). The expectation is that in this way a routine and systematic evaluation of model results can be made more efficient, thereby enabling scientists to focus on developing more innovative methods of analysis rather than constantly having to "reinvent the wheel".

In parallel to standardization of model output, the ESGF also hosts observations for Model Intercomparison Projects (obs4MIPs) and reanalyses data (ana4MIPs). obs4MIPs provides open access data sets of satellite data that are comparable in terms of variables, temporal and spatial frequency, and periods to CMIP model output (Taylor et al., 2012). The ESMValTool utilizes these observations and reanalyses from ana4MIPs plus additionally available observations in order to evaluate the models performance. In many diagnostics and metrics, more than one observational data set or meteorological reanalysis is used to assess uncertainties in observations.

\subsection{Objectives and Approach}

The main idea of the ESMValTool is to provide a broad suite of diagnostics which can be performed easily when new model simulations are run. The suite of diagnostics needs to be broad enough to reflect the diversity and complexity of Earth System Models, but must also be robust enough to be run routinely or semi-operationally.

In order the address these challenging objectives the ESMValTool is conceived as a framework which allows community contributions to be bound into a coherent framework.

\subsection{Architecture}

Figure 1 shows a schematic of the ESMValTool architecture: the workflow manager (controlled by the Python script "main.py") runs a set of diagnostics on data provided by, for instance, a data archive. The configuration and the settings of each diagnostic are specified in namelists read and passed to the diagnostics by the workflow manager. The results which typically comprise of netCDF files and/or plots are stored in 
output folders along with log-files summarizing the data used, references, and technical details to ensure traceability and reproducibility of the results.

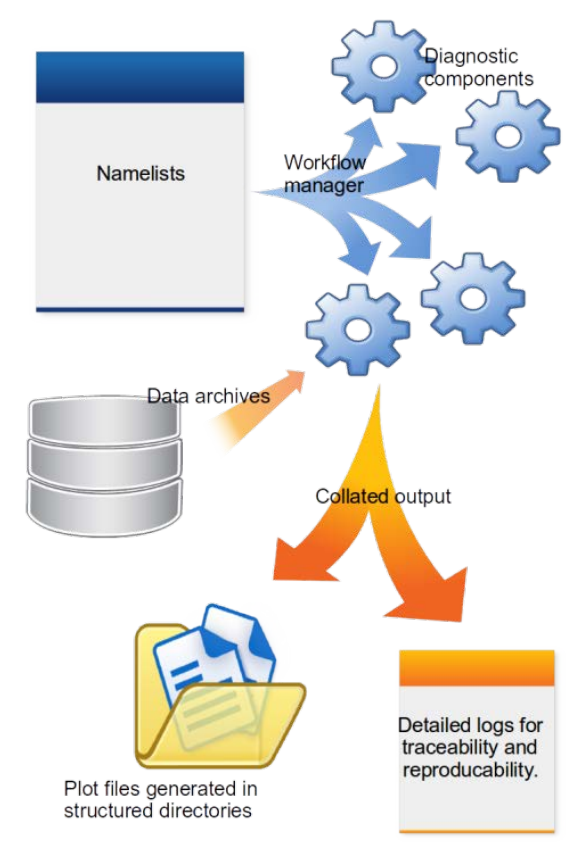

Figure 1 Schematic of the system architecture. The workflow manager (main.py) passes information to the diagnostics; results and log-files are written to dedicated folders.

\section{Software installation}

\subsection{Prerequisites}

The ESMValTool has the following software requirements (note that specific diagnostics might require additional software packages):

- Unix(-like) operating system

- Python version 2.7.x for running the Python script main.py; Some diagnostics written in Python (e.g., TropicalVariability) require installation of additional Python packages such as the Geometry Engine (GEOS), scientificpython, netCDF4, and cdo (runoff_et), e.g.:

- conda install basemap

- conda install --channel https://conda.anaconda.org/Clyde_Fare scientificpython

- conda install netcdf4

- conda install --channel https://conda.anaconda.org/auto cdo

It is strongly recommended to use the Python distribution Anaconda (https://www.continuum.io/), as it allows the user to install additional Python libraries and extensions in a simple way and without modifying the installed Python distribution (i.e., without root permissions). The installation instructions for the additional Python packages listed above are given for Anaconda. 
- NCAR Command Language (NCL 2014) version 6.2 or higher to run the quality check and reformat routines processing all input files. See the control flow description on reformat_default in Table S11 for details.

- The statistical computing software $R$ to run diagnostics written in $R$. A working installation of $R$ and the executable Rscript in the default search path are required. In addition, the netCDF for $R$ libraries (ncdf / ncdf4) are needed. Currently, only the diagnostic Standardized Precipitation index (SPI) (see Annex C)" requires $R$. More diagnostics written in $R$ might be added in the future.

- Input files in netCDF with required attributes and dimension names. Valid input files are:

- a CMIP or similarly standardized format using a CMIP5 table, or with discrepancies that can be handled via the definitions in the files reformat_scripts/recognized_units.dat and reformat_scripts/recognized_units.dat, respectively.

- any input file with a (user-)supplied reformat routine that converts the input data during runtime, see the control flow description on reformat_EMAC in Table S11 for details

- Common GNU utilities such as "wc", "date", "basename", and "more", which are usually part of the standard Linux distribution

\subsection{Obtaining the source code}

The ESMValTool is available at http://www.esmvaltool.org/ via a tar-file ("tar-ball"). The ESMValTool is released under the Apache License, version 2.0 and citation of the ESMValTool paper ("Software Documentation Paper") is kindly requested upon use alongside with the software doi (doi:10.17874/ac8548f0315) and version number:

Eyring et al., ESMValTool (v1.0) - a community diagnostic and performance metrics tool for routine evaluation of Earth System Models in CMIP, Geosci. Model Dev., 2016.

Besides the above citation, users are kindly asked to register any journal articles (or other scientific documents) that use the software at the ESMValTool webpage (http://www.esmvaltool.org/). Citing the Software Documentation Paper and registering your paper(s) will serve to document the scientific impact of the Software, which is of vital importance for securing future funding. You should consider this an obligation if you have taken advantage of the ESMValTool, which represents the end product of considerable effort by the development team.

The ESMValTool will be further developead in a version controlled repository (see section 12.1) that is accessible only to the development team. In addition to using the software, we would therefore like to encourage the community to join the Software Development Team and to contribute additional diagnostics and performance metrics or other software improvements. Contributing back the new diagnostics and performance metrics or other software improvements will help to enhance the capability of the Software, which is of vital importance for securing future funding. You should consider this an obligation if you have taken advantage of the Software, which represents a product of considerable effort by the development team.

A wiki page (see section 12.3) that describes ongoing developments is available. Interested users and developers are welcome to contact the core development team (see section 9). 


\subsection{Software installation}

The tar-ball can be unpacked with the standard tar command, e.g.,

$$
\text { tar-xvf ESMValTool_v1.0.tar }
$$

\section{ESMValTool namelists}

The ESMValTool namelists are the "control centers" acting as interfaces between the user and the various scripts and configuration files that make up the ESMValTool. A namelist specifies a list of diagnostics to run, global flags and a list of models and observations that are used within the diagnostics. Namelists are text files written in XML (EXtensible Markup Language) [XML]. As a simple text file, the XML-namelist can be easily modified by the user.

For any given namelist "namelist.xml", the ESMValTool is invoked from the command line via (see also section 6):

\section{python main.py $\mathrm{nml} /$ namelist.xml}

The Python "workflow manager" main.py will parse the namelist (namelist.xml) and call all diagnostic scripts listed in the namelist. This sequence is schematically depicted in Figure 2 and involves the following steps:

1. parse the namelist

2. identify the input files on the file system

3. run an NCL script to check and reformat the input files

4. if needed, run a NCL script to compute derived variables such as, for instance, climate indices

5. run the diagnostic script (NCL/Python/R/etc.)

6. repeat previous steps until all diagnostics listed in the namelist are processed 


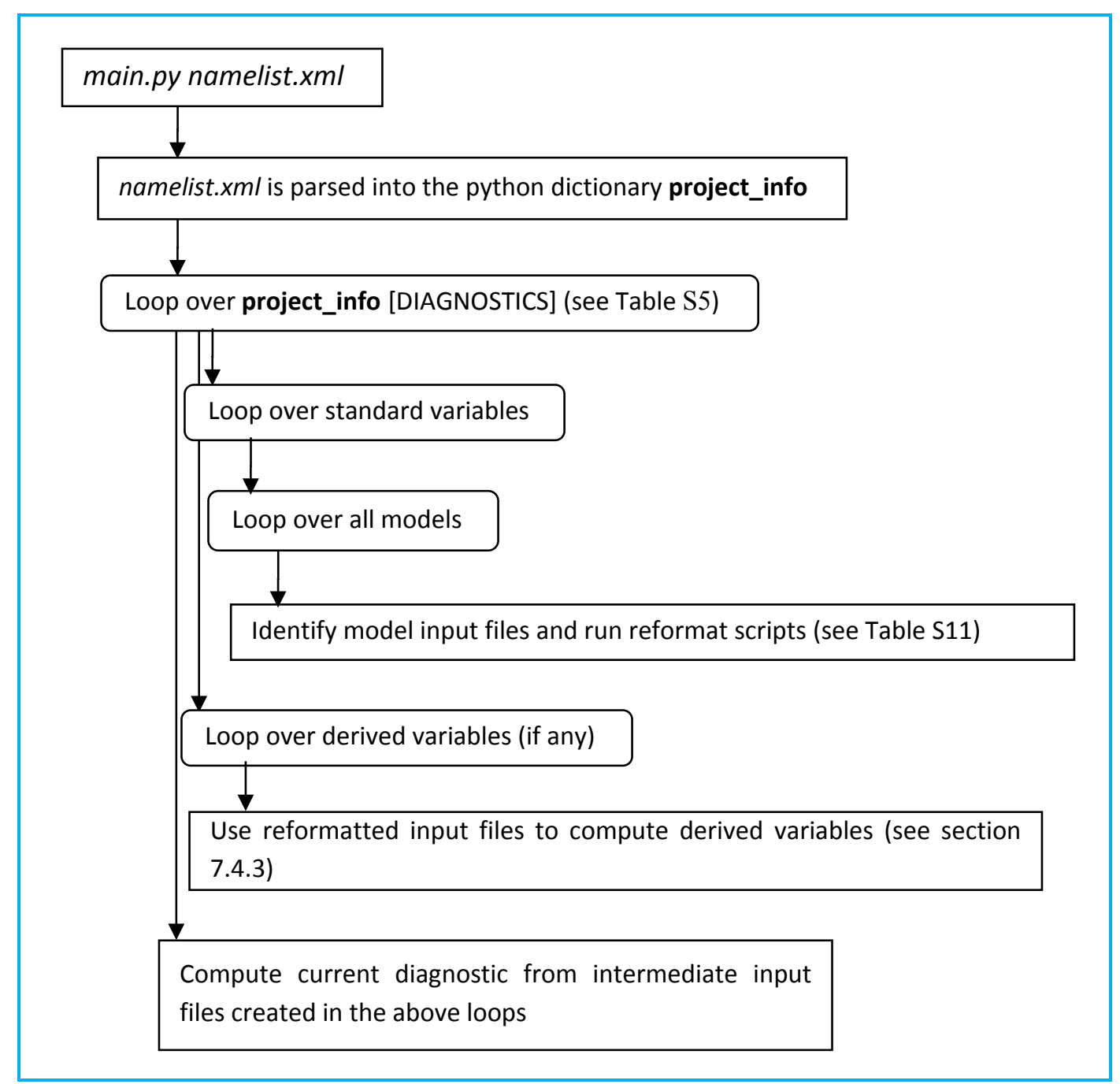

Figure 2 ESMValTool control flow.

The script main.py processes the information in the XML namelist to be used by each of the supported programming languages (currently NCL, Python and R) used for the diagnostic scripts. This means that different diagnostics, even if implemented in different programming languages, can be called within the same namelist. Any changes to the settings of the namelist will passed to each diagnostic script.

Note that the coupling between the namelist and the diagnostic scripts is "loose". The Python workflow manager main.py passes all information in the namelist to the target diagnostic script, e.g., via intermediate files or environment variables, but it is up to the diagnostic script to act on that information. 


\section{Basic structure of a namelist}

<GLOBAL>

controls the general settings (see Table S1) ; see section 3.1, "More on the <GLOBALS>-tag" below for details

</GLOBAL>

$<$ MODELS>

defines the models/observations and years to be processed and their pathnames; see the section 3.2, "More on the <MODELS>-tag" below for details

\section{$</$ MODELS $>$}

\section{<DIAGNOSTIC>}

defines which diagnostics are run (see Table S5); each diagnostic is enclosed in an opening <diag> and closing </diag >-tag; see the section 0 , "More on the <DIAGNOSTICS $>$-tag" below for details

$</$ DIAGNOSTIC>

Please note that the "loose coupling" described above applies particularly to the settings defined in the two elements $<$ GLOBAL $>$ and $<$ DIAGNOSTIC $>$.

\subsection{More on the <GLOBAL >-tag}

Table S1 summarizes the tags defined in the $<$ GLOBAL $>$ section of the namelist. Some of these tags (e.g., regridding_dir) are specific to some diagnostics and not generally defined in all namelists.

Table S1 Tags of the $<$ GLOBAL $>$ section of the namelist. Note that not all tags might be used by a diagnostic.

\begin{tabular}{lll}
\hline Name & Type & Description \\
\hline climo_dir & String & Path for intermediate files (netCDF) \\
exit_on_warning & boolean & Stop on warnings \\
force_calc & boolean & Force diagnostic specific files to be recreated \\
force_gradecalc & Boolean & Force recalculation of model grading (perfmetrics) \\
force_processing & boolean & Force certain intermediate files (netCDF) to be recreated instead of using cached files \\
force_taylorcalc & boolean & Force recalculation of data for Taylor plot (perfmetrics) \\
max_data_blocksize & integer & Currently not used \\
\hline max_data_filesize & integer & Limits internal memory handling in some core NCL scripts \\
\hline output_file_type & String & File format of plots (ps, pdf, eps, png); not all formats supported by all diagnostic scripts \\
plot_dir & String & Output path for plots \\
read_from_vault & boolean & Retrieve computed diagnostic fields from netCDF \\
\hline regridding_dir & String & Path for intermediate files used by NCL regridding routines \\
show_debuginfo & String & Generate a second version of each figure with explanatory text overlayed \\
verbosity & integer & Verbosity level (0 = minimum output, 4=maximum output) \\
write_netcdf & boolean & Write results to netCDF file \\
\hline write_plot_vars & boolean & Currently not used \\
write_plots & boolean & Produce plots \\
wrk_dir & String & Output path for data (netCDF, acknowledgements) \\
\hline
\end{tabular}




\subsection{More on the $<$ MODELS $>$-tag}

Each data set is specified by a <model $>$ line with the first entry of each model line being the "project specifier" (see Table S2). The project specifier refers to a Python class that is used to parse the model line in the namelist. For example, a model line with the "CMIP5" specifier looks like:

$<$ model $>$ CMIP5 name mip experiment ensemble start-year end-year path </model $>$

- Optionally, the element "mip" can be replaced with "MIP_VAR_DEF" if the tag "MIP" is specified in the $<$ variable $>$ tag (see Table S4), e.g.:

$<$ variable MIP="cfDay" $>$ rlut $</$ variable $>$

$<$ model> CMIP5_ETHZ MPI-ESM-LR MIP_VAR_DEF amip r1i1p1 19801985

@\{MODELPATH $\} / E T H Z \_C M I P 5 /</$ model $>$

- The element "experiment" can be replaced with "EXP_VAR_DEF" if the tag "EXP" is specified in the < variable $>$ tag (see Table S4), e.g.:

$<$ variable MIP="Omon" $\mathbf{E X P}=$ "esmHistorical" $>$ fgco2 </variable $>$

$<$ model> CMIP5_ETHZ NorESM1-ME MIP_VAR_DEF EXP_VAR_DEF r1i1p1 19602005

@\{MODELPATH $\} / E T H Z \_C M I P 5</$ model $>$

The project specifier "CMIP5" will search for files in "path" with filenames matching the pattern

*_mip_name_experiment_ensemble_*

Here, the leading asterisk is a placeholder for the variable, which is defined in the $<$ DIAGNOSTICS $>$-tag (see below), the trailing asterisk is a placeholder for the start/end date of the data set. This naming convention conforms to the syntax used for CMIP5 DRS filenames (as implied by the project specifier name). By implementing their own project specifier classes into the Python code (interface_scripts/projects.py), the user can handle data sets that follow different file naming conventions or require additional information to be passed along in addition to the filename. Table S2 gives a summary of the available project specifiers and arguments to be used in each $<$ model $>$ line.

Note: Examples for the most commonly used project specifiers CMIP5, CMIP5_ETHZ, OBS, and obs4mips as well as downloading instructions and information on the required local directory structure for the model / observational data can be found in section 6.1.

The < model $>$-tag may also take the optional attribute "id":

$$
<\text { model id="string" }>
$$

Example:

<model id="ERAINT"> OBS ERA-Interim reanaly $120032004 @\{O B S P A T H\} / T i e r 3 / E R A-I n t e r i m$ $<$ model $>$

The attribute id specifies a string that can be used to refer to the model in other places of the namelist. Table S3 gives a summary of valid attributes in $<$ model $>$-tags. 
Table S2 Project specifiers and corresponding arguments.

\begin{tabular}{|c|c|c|c|c|c|c|c|c|}
\hline project specifier & $\begin{array}{c}\text { argument } \\
1\end{array}$ & $\begin{array}{c}\text { argument } \\
2\end{array}$ & $\begin{array}{c}\text { argument } \\
\mathbf{3}\end{array}$ & $\begin{array}{c}\text { argument } \\
4\end{array}$ & $\begin{array}{c}\text { argument } \\
5\end{array}$ & $\begin{array}{c}\text { argument } \\
6\end{array}$ & $\begin{array}{c}\text { argument } \\
7\end{array}$ & $\begin{array}{c}\text { argument } \\
8 \\
\end{array}$ \\
\hline ana4mips & Name & table & $\begin{array}{l}\text { experime } \\
\text { nt }\end{array}$ & ensemble & realm & start year & end year & path \\
\hline CCMVal & name & case & ensemble & start year & end year & path & - & - \\
\hline CCMVal1 & name & name & ensemble & start year & end year & path & - & - \\
\hline CCMVal2 & name & $\begin{array}{l}\text { case } \\
\text { name } \\
\text { case } \\
\text { name }\end{array}$ & ensemble & start year & end year & path & - & - \\
\hline $\begin{array}{l}\text { CMIP5 } \\
\text { CMIP5_ETHZ } \\
\text { CMIP5_gridfile } \\
\text { CMIP5_SMHI }\end{array}$ & $\begin{array}{l}\text { name } \\
\text { name } \\
\text { name } \\
\text { name }\end{array}$ & $\begin{array}{l}\operatorname{mip} \\
\operatorname{mip} \\
\operatorname{mip} \\
\operatorname{mip}\end{array}$ & $\begin{array}{l}\text { experime } \\
\text { nt } \\
\text { experime } \\
\text { nt } \\
\text { experime } \\
\text { nt } \\
\text { experime } \\
\text { nt }\end{array}$ & $\begin{array}{l}\text { ensemble } \\
\text { ensemble } \\
\text { ensemble } \\
\text { ensemble }\end{array}$ & $\begin{array}{l}\text { start year } \\
\text { start year } \\
\text { start year } \\
\text { start year }\end{array}$ & $\begin{array}{l}\text { end year } \\
\text { end year } \\
\text { end year } \\
\text { end year }\end{array}$ & $\begin{array}{l}\text { Path } \\
\text { path } \\
\text { path } \\
\text { frequency }\end{array}$ & $\begin{array}{l}- \\
\text { gridfile } \\
\text { path }\end{array}$ \\
\hline ECEARTH & Name & $\begin{array}{l}\text { experime } \\
\text { nt }\end{array}$ & ensemble & start year & end year & path & - & - \\
\hline EMAC & Name & ensemble & start year & end year & path & - & - & - \\
\hline $\mathrm{GO}$ & name & table & $\begin{array}{l}\text { experime } \\
\text { nt }\end{array}$ & ensemble & start year & end year & path & - \\
\hline GO_gridfile & name & table & $\begin{array}{l}\text { experime } \\
\text { nt }\end{array}$ & ensemble & start year & end year & path & - \\
\hline $\begin{array}{l}\text { MiKlip } \\
\text { MiKlip_baseline0 }\end{array}$ & $\begin{array}{l}\text { name } \\
\text { name }\end{array}$ & $\begin{array}{l}\text { table } \\
\text { table }\end{array}$ & $\begin{array}{l}\text { experime } \\
\text { nt } \\
\text { experime } \\
\text { nt }\end{array}$ & $\begin{array}{l}\text { ensemble } \\
\text { ensemble }\end{array}$ & $\begin{array}{l}\text { realm } \\
\text { realm }\end{array}$ & $\begin{array}{l}\text { start year } \\
\text { start year }\end{array}$ & $\begin{array}{l}\text { end year } \\
\text { end year }\end{array}$ & $\begin{array}{l}\text { path } \\
\text { path }\end{array}$ \\
\hline OBS & name & $\begin{array}{l}\text { case } \\
\text { name }\end{array}$ & ensemble & start year & end year & Path & - & - \\
\hline OBS_gridfile & name & $\begin{array}{l}\text { case } \\
\text { name } \\
\text { (insitu, } \\
\text { sat, } \\
\text { ground, } \\
\text { reanaly) }\end{array}$ & ensemble & start year & end year & path & gridfile & - \\
\hline obs4mips & Name & $\begin{array}{l}\text { process } \\
\text { level }\end{array}$ & ensemble & start year & end year & path & - & - \\
\hline
\end{tabular}

Table S3 Optional attributes of the $<$ model $>$ tag.

\begin{tabular}{lll}
\hline Name & Type & Description \\
\hline id & String & Define a name used to refer to the model data in other parts of the namelist \\
\hline
\end{tabular}

Table S4 Optional attributes of the $<$ variable $>$ tag.

\begin{tabular}{lll}
\hline Name & Type & Description \\
\hline exclude & String & Model (id) to exclude from processing \\
EXP & String & $\begin{array}{l}\text { Define a name used to the CMIP5 experiment, e.g., } \\
\text { Define a name used to refer to the CMIP5 data stream, e.g., "Amon", "Omon", "day", } \\
\text { "fx"; to be used in combination with "MIP_VAR_DEF" replacing the CMIP5 stream in the } \\
\text { definition of a }<\text { model } \text { tag. }\end{array}$ \\
ref_model & Define a reference model (model id) \\
\hline
\end{tabular}




\subsection{More on the $<$ DIAGNOSTICS $>$-tag}

Each < diag $>$ entry refers to one or several scripts in the folder diag_scripts/ complemented by a variable name (see Table S8 for a list of variables; please note that the list of variables is constantly extended and check the ESMValTool wiki page (see section 12.3) for the most recent list) and the corresponding (input) field type (see Table S7). Optionally the $<$ diag $>$-tag may contain additional $<$ model $>$-tags; these data sets will be processed only by the diagnostic(s) listed in the current $<$ diag $>$ entry. In this way it is possible to define a set of models to be analyzed by all diagnostics in the namelist (in the $<$ MODELS $>$ section) and a set of models to be analyzed only by specific diagnostics (in the $<$ diag $>$ section). Available $<$ diag $>$-tags are listed in Table S5, their optional attributes in Table S6.

Table S5 Tags of the $<$ diag $>$ section within the $<$ DIAGNOSTICS $>$ section of the namelist. There are no default values.

\begin{tabular}{|c|c|c|}
\hline Name & Type & Description \\
\hline description & string & 1-line description / title of the diagnostic \\
\hline variable_def_dir & string & Path for the variable-specific configuration file (usually variable_defs) \\
\hline variable & string & $\begin{array}{l}\text { Variable name: a script with the same name (variable_defs/<variable }>\text {.ncl) defines the variable to } \\
\text { process (see Table } \mathrm{S} 8 \text { for a list of variables) including possible preprocessing (e.g., calculating } \\
\text { derived variables). Variable scripts should be located in the local folder variable_defs and written } \\
\text { in NCL. Even though the variable scripts are written in } \mathrm{NCL} \text { all meta data defined in the scripts are } \\
\text { passed on to the target diagnostic script regardless of the used language (via variable attributes). } \\
\text { If multiple variables need to be passed on to a diagnostic script, multiple <variable>-tags have to } \\
\text { be defined. }\end{array}$ \\
\hline field_type & string & $\begin{array}{l}\text { Type of input field (see Table S7) that can be used by the diagnostic scripts. If multiple }<\text { variable }>\text { - } \\
\text { tags are defined a single (which is then applied to all) or an equal number of }<\text { field type }>\text {-tags has } \\
\text { to be defined. }\end{array}$ \\
\hline diag_script_cfg_dir & string & Path for diagnostic script configuration file \\
\hline diag_script & string & $\begin{array}{l}\text { Name of diagnostic script; the script can be written in any language currently supported by } \\
\text { ESMValTool (NCL, R and Python) and has to be located in the local folder diag_scripts. The } \\
\text { settings defined in the diagnostic script configuration file defined by the diag_script cfg attribute } \\
\text { is loaded at the beginning of the diagnostic script. }\end{array}$ \\
\hline model (optional) & string & $\begin{array}{l}\text { Additional data sets specific for this < diag >-section. Data sets defined here will be processed in } \\
\text { addition to the ones defined in the MODELS section (see above) but will be ignored by other } \\
\text { <diag>-sections. }\end{array}$ \\
\hline
\end{tabular}

Table S6 Optional attributes of selected tags in the $<$ diag $>$ section.

\begin{tabular}{|c|c|c|c|}
\hline Name & Type & Parent tag & Description \\
\hline ref_model & string & $<$ variable> & $\begin{array}{l}\text { Defines this data set as the reference data set within the diagnostic. The string } \\
\text { ref_model refers to either the model name, as specified in Table S2, or the model } \\
\text { attribute id as specified in Table S3. Note that because both model and observational } \\
\text { data sets are specified via the <model>-tag any of them can be used as a reference } \\
\text { data set. }\end{array}$ \\
\hline \multirow[t]{2}{*}{ exclude } & string & <variable> & $\begin{array}{l}\text { When using more than one variable corresponding to different observational data sets } \\
\text { (e.g., precipitation and skin temperature), it is necessary to use this attribute to match } \\
\text { which variable goes with which data set, e.g., pr with TRMM and ts with HadISST using, }\end{array}$ \\
\hline & & & $\begin{array}{l}<\text { variable ref_model="trmm" exclude="hadisst"> pr ... } \\
<\text { variable ref_model="hadisst" exclude="trmm"> ts ... }\end{array}$ \\
\hline $\operatorname{cfg}$ & string & $<$ diag_script $>$ & Configuration file for the diagnostic script \\
\hline
\end{tabular}

Table S7 Field types.

\begin{tabular}{ll}
\hline Name & Description \\
\hline T2Ms & Monthly-mean 2d atmosphere or land surface data (longitude, latitude, time:month) \\
T3M & Monthly-mean 3d atmosphere data (longitude, latitude, pressure, time:month) \\
T2Mz & Monthly-mean zonal mean 2d atmosphere or land surface data (longitude, pressure, time:month) \\
\hline
\end{tabular}




\begin{tabular}{ll}
\hline T1Ms & Monthly-mean 1d atmosphere or land surface data on a certain pressure level (latitude, time:month) \\
T2Ds & Daily-mean 2d atmosphere data (longitude, latitude, time:day) \\
T3D & Daily-mean 3d atmosphere data (longitude, latitude, pressure, time:day) \\
T2Dz & Daily-mean zonal mean 2d atmosphere data (latitude, pressure, time:month) \\
T2Is & Daily instantaneous 2d atmosphere data for all years (longitude, latitude, time:day) \\
T3I & Daily-instantaneous 3d atmosphere data for selected years (longitude, latitude, model level, time:day) \\
T2Iz & Daily instantaneous zonal mean 2d atmosphere data for all years (latitude, pressure, time:day) \\
T1lz & Daily instantaneous 1d field for all years (latitude-pressure, time:day) \\
TOI & Daily instantaneous Od field for all years (time:day) \\
TOAs & Annual-mean 0d atmosphere or land surface data on a certain pressure level (latitude, time:year) \\
F2Ms & Constant 2d land surface data (latitude, longitude) \\
TO2Ms & Monthly-mean 2d ocean or sea ice data (longitude, latitude, time:month) \\
TO3M & Monthly-mean 3d ocean or sea ice data (longitude, latitude, model level, time:month) \\
\hline
\end{tabular}

Table S8 Variable definition scripts.

\begin{tabular}{|c|c|}
\hline Script name & Description \\
\hline albisccp.ncl & ISCCP-like cloud albedo \\
\hline chl.ncl & Chlorophyll mass concentration at the surface (ocean) \\
\hline clivi.ncl & Vertically integrated cloud ice \\
\hline cl.ncl & Cloud area fraction $(3 \mathrm{~d})$ \\
\hline clt.ncl & Total cloud fraction \\
\hline cltisccp.ncl & ISCCP-like total cloud fraction \\
\hline clwvi.ncl & Vertically integrated total cloud water (ice + liquid) \\
\hline co2flux.ncl & Sum of land and ocean carbon fluxes \\
\hline concbc.ncl & $\mathrm{BC}$ concentration \\
\hline concbcSTP.ncl & EMAC aerosol variable \\
\hline conccnmode.ncl & EMAC aerosol variable \\
\hline conccnSTPd120.ncl & EMAC aerosol variable \\
\hline conccnSTPd14.ncl & EMAC aerosol variable \\
\hline conccnSTPd3.ncl & EMAC aerosol variable \\
\hline conccnSTPd5.ncl & EMAC aerosol variable \\
\hline cSoil.ncl & Carbon mass in soil pool \\
\hline cumnbp.ncl & Cumulated NBP \\
\hline cVeg.ncl & Carbon mass in vegetation \\
\hline diamcnmode.ncl & EMAC aerosol variable \\
\hline et.ncl & Evapotranspiration \\
\hline evspsbl.ncl & Evaporation \\
\hline fgco2.ncl & Surface downward $\mathrm{CO}_{2}$ flux (ocean) \\
\hline gpp.ncl & Carbon mass flux out of atmosphere due to gross primary production on land \\
\hline hfds.ncl & Downward heat flux at sea surface \\
\hline hfls.ncl & Surface upward latent heat flux (includes both evaporation and sublimation) \\
\hline hfss.ncl & Surface upward sensible heat flux \\
\hline hus.ncl & Specific humidity \\
\hline huss.ncl & Surface specific humidity \\
\hline ita.ncl & Depth weighted temperature (ocean, $730 \mathrm{~m}$ ) \\
\hline lai.ncl & Leaf area index \\
\hline LW_CRE.ncl & Longwave cloud radiative forcing \\
\hline Iwp.ncl & Vertically integrated cloud water (liquid only) \\
\hline mlotst.ncl & Ocean mixed layer thickness \\
\hline mmraer.ncl & EMAC aerosol variable \\
\hline mmrbcfree.ncl & EMAC aerosol variable \\
\hline mmrbc.ncl & $\mathrm{BC}$ mass mixing ration \\
\hline mrro.ncl & Total runoff \\
\hline mrso.ncl & Soil moisture content \\
\hline msftmyz.ncl & Ocean meridional overturning mass streamfunction \\
\hline MyVar.ncl & Template \\
\hline nbp.ncl & Carbon mass flux out of atmosphere due to net biospheric production on land \\
\hline NET_CRE.ncl & Net cloud forcing \\
\hline $02 . \overline{\mathrm{ncl}}$ & $\mathrm{O}_{2}$ (ocean) \\
\hline od550aer.ncl & Aerosol optical depth (550 nm) \\
\hline pctisccp.ncl & ISCCP-like cloud top height \\
\hline
\end{tabular}




\begin{tabular}{|c|c|}
\hline pr-mmday.ncl & Precipitation (total) in $\mathrm{mm}$ per day \\
\hline pr.ncl & Precipitation (total) \\
\hline psl.ncl & Surface pressure \\
\hline rldscs.ncl & Surface downwelling longwave flux (clear sky) \\
\hline rlds.ncl & Surface downwelling longwave flux (all sky) \\
\hline rlus.ncl & Surface upwelling longwave flux \\
\hline rlutcs.ncl & TOA outgoing clear-sky longwave radiation \\
\hline rlut.ncl & TOA outgoing all-sky longwave radiation \\
\hline rsdscs.ncl & Surface downwelling shortwave flux (clear_sky) \\
\hline rsds.ncl & Surface downwelling shortwave flux (all sky) \\
\hline rsutcs.ncl & TOA outgoing clear-sky shortwave radiation \\
\hline rsut.ncl & TOA outgoing all-sky shortwave radiation \\
\hline sconcbc.ncl & BC surface concentration \\
\hline sconccl.ncl & $\mathrm{Cl}^{-}$surface concentration (aerosol) \\
\hline sconcna.ncl & $\mathrm{Na}^{+}$surface concentration (aerosol) \\
\hline sconcnh4.ncl & $\mathrm{NH}_{4}$ surface concentration \\
\hline sconcno3.ncl & $\mathrm{NO}_{3}$ surface concentration \\
\hline sconcoa.ncl & Organic aerosol (OA) surface concentration \\
\hline sconcpm10.ncl & $\mathrm{PM}_{10}$ surface concentration \\
\hline sconcpm 2 p5.ncl & $\mathrm{PM}_{2.5}$ surface concentration \\
\hline sconcso4.ncl & $\mathrm{SO}_{4}$ surface concentration \\
\hline sfcWind.ncl & Near-surface wind speed \\
\hline sftlf.ncl & Land fraction \\
\hline sic.ncl & Sea ice area fraction \\
\hline sit.ncl & Sea ice thickness \\
\hline snc.ncl & Fraction of grid cell covered by snow on land \\
\hline snd.ncl & Surface snow thickness \\
\hline snw.ncl & Mass of snow on land \\
\hline so.ncl & Sea water salinity \\
\hline sos.ncl & Sea surface salinity \\
\hline spco2.ncl & $\mathrm{pCO}_{2}$ (ocean) \\
\hline stratospheric_column.ncl & Stratospheric ozone column \\
\hline SW_CRE.ncl & Shortwave cloud radiative forcing \\
\hline talk.ncl & Total alkalinity (ocean) \\
\hline ta.ncl & Air temperature \\
\hline tas.ncl & Near-surface air temperature \\
\hline tas-degC.cnl & Near-surface air temperature in degrees Centigrade \\
\hline tauu.ncl & Surface eastward wind stress \\
\hline tauv.ncl & Surface northward wind stress \\
\hline tauw.ncl & Surface wind stress \\
\hline theta-850.ncl & Potential temperature at $850 \mathrm{hPa}$ \\
\hline theta.ncl & Potential temperature \\
\hline to.ncl & Sea water temperature \\
\hline tos.ncl & Sea surface temperature \\
\hline total_column.ncl & Total ozone column \\
\hline toz.ncl & Total ozone column (alternative name) \\
\hline tro3.ncl & Ozone volume mixing ratio \\
\hline tropospheric_column.ncl & Tropospheric ozone column \\
\hline tropoz.ncl & Tropospheric ozone column (alternative name) \\
\hline ts.ncl & Skin temperature \\
\hline ua-1000.ncl & Wind u-component at $1000 \mathrm{hPa}$ \\
\hline ua-200-850.ncl & Wind u-component at $200 \mathrm{hPa}$ and at $850 \mathrm{hPa}$ (monsoon diagnostics) \\
\hline ua-200.ncl & Wind u-component at $200 \mathrm{hPa}$ \\
\hline ua-700.ncl & Wind u-component at $700 \mathrm{hPa}$ \\
\hline ua-850.ncl & Wind u-component at $850 \mathrm{hPa}$ \\
\hline ua-925.ncl & Wind u-component at $925 \mathrm{hPa}$ \\
\hline ua.ncl & Wind u-component \\
\hline uo.ncl & Sea water $x$ velocity \\
\hline va-200-850.ncl & Wind v-component at $200 \mathrm{hPa}$ and at $850 \mathrm{hPa}$ (monsoon diagnostics) \\
\hline va-200.ncl & Wind v-component at $200 \mathrm{hPa}$ \\
\hline va-700.ncl & Wind v-component at $700 \mathrm{hPa}$ \\
\hline va-850.ncl & Wind v-component at $850 \mathrm{hPa}$ \\
\hline va-925.ncl & Wind v-component at $925 \mathrm{hPa}$ \\
\hline va.ncl & Wind v-component \\
\hline
\end{tabular}




\begin{tabular}{|c|c|}
\hline vmrc2h4.ncl & EMAC chemistry variable \\
\hline vmrc2h6.ncl & EMAC chemistry variable \\
\hline vmrc3h6.ncl & EMAC chemistry variable \\
\hline vmrc3h8.ncl & EMAC chemistry variable \\
\hline vmrch3coch3.ncl & EMAC chemistry variable \\
\hline vmrco_alt.ncl & EMAC chemistry variable \\
\hline vmrco_azr.ncl & EMAC chemistry variable \\
\hline vmrco_chr.ncl & EMAC chemistry variable \\
\hline vmrco_eic.ncl & EMAC chemistry variable \\
\hline vmrco_gmi.ncl & EMAC chemistry variable \\
\hline vmrco_hpb.ncl & EMAC chemistry variable \\
\hline vmrco_lef.ncl & EMAC chemistry variable \\
\hline vmrco_mlo.ncl & EMAC chemistry variable \\
\hline vmrco.ncl & $\mathrm{CO}$ volume mixing ratio \\
\hline vmrco_nwr.ncl & EMAC chemistry variable \\
\hline vmrh2o.ncl & EMAC chemistry variable \\
\hline vmrnox.ncl & $\mathrm{NO}_{\mathrm{x}}$ volume mixing ratio \\
\hline vmro3.ncl & $\mathrm{O}_{3}$ volume mixing ratio \\
\hline vmro3_NE.ncl & EMAC chemistry variable \\
\hline vmro3_NHext.ncl & EMAC chemistry variable \\
\hline vmro3_SHext.ncl & EMAC chemistry variable \\
\hline vmro3_Trop.ncl & EMAC chemistry variable \\
\hline vo.ncl & Sea water y velocity \\
\hline wfpe-mmday & Water flux from precipitation and evaporation in $\mathrm{mm} \mathrm{day}^{-1}$ \\
\hline wfpe.ncl & Water flux from precipitation and evaporation \\
\hline zg.ncl & Geopotential height \\
\hline
\end{tabular}

Typically, all namelists are stored in the folder $\mathrm{nml}$, the naming convention is namelist_xxx.xml with "xxx" being the name of the diagnostic and/or a description of the purpose of the namelist:

\section{- For papers}

$\mathrm{xxx}=$ SurnameYearJournalabbreviation (e.g., stocker12jgr, stocker12sci1, stocker12sci2).

\section{- For copies of reports that are not publicly available}

$\mathrm{xxx}=$ OrgYearTitleabbrev (e.g., unep10water, unep11gap, roysoc09geoengineering).

- For grouped sets of diagnostics and performance metrics that do not follow a published paper or report

$\mathrm{xxx}=$ an intuitive name describing the scientific topic (e.g., aerosol, MyDiag, SAMonsoon, SeaIce)

\subsection{Namelist configuration file}

The user can define base path names in a namelist configuration file and refer to them in the actual namelist file. The configuration file such as, for instance, config_private.xml has the following structure:

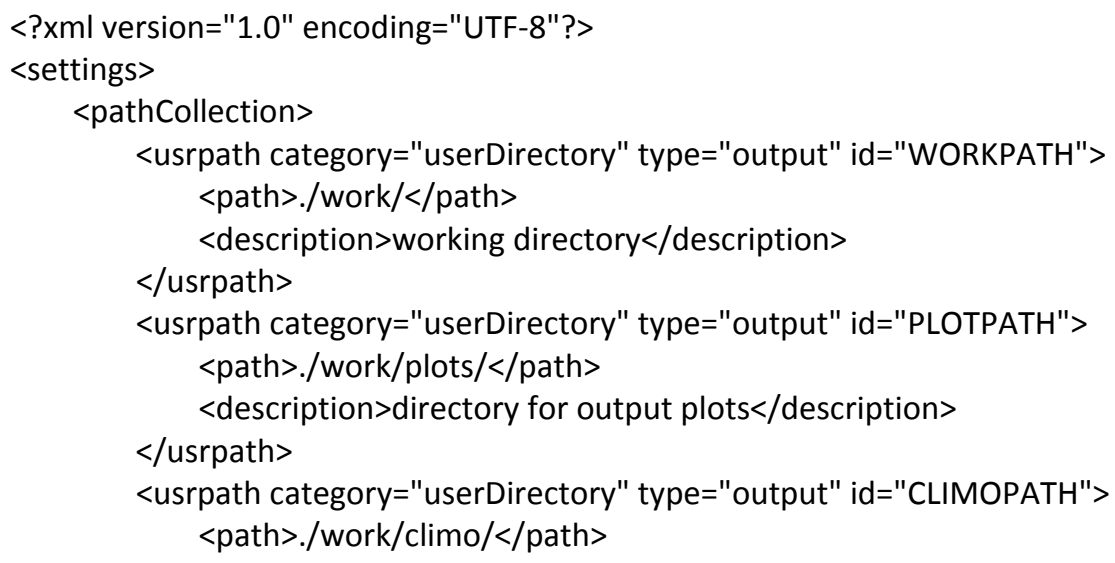




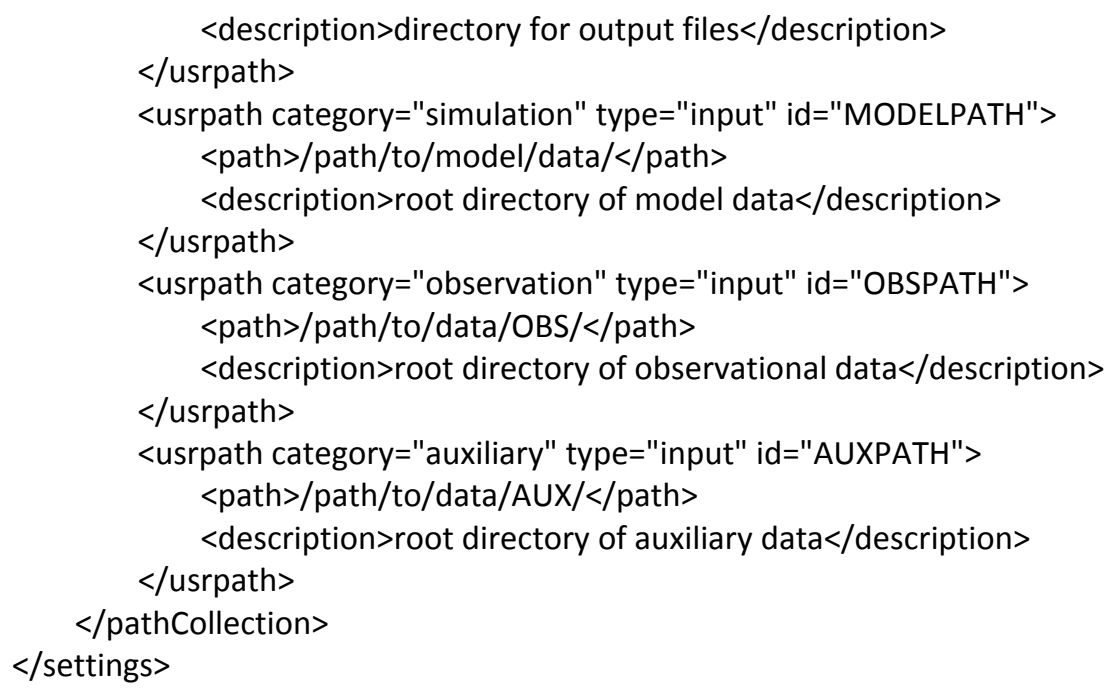

Inside the namelist file the configuration file can be included in the following way:

<include href="config_private.xml"/>

and referred to with the syntax:

$@\{$ id-of-the-usrpath $\}$

Note: alternatively, explicitely defined pathnames can be used at any time.

\subsection{Standard header for the namelist}

For the sake of documentation, standard headers are defined and applied to all namelists and scripts in the ESMValTool. This is a template of the standard header for the main namelist. The parts in red are the ones to be modified by the author.

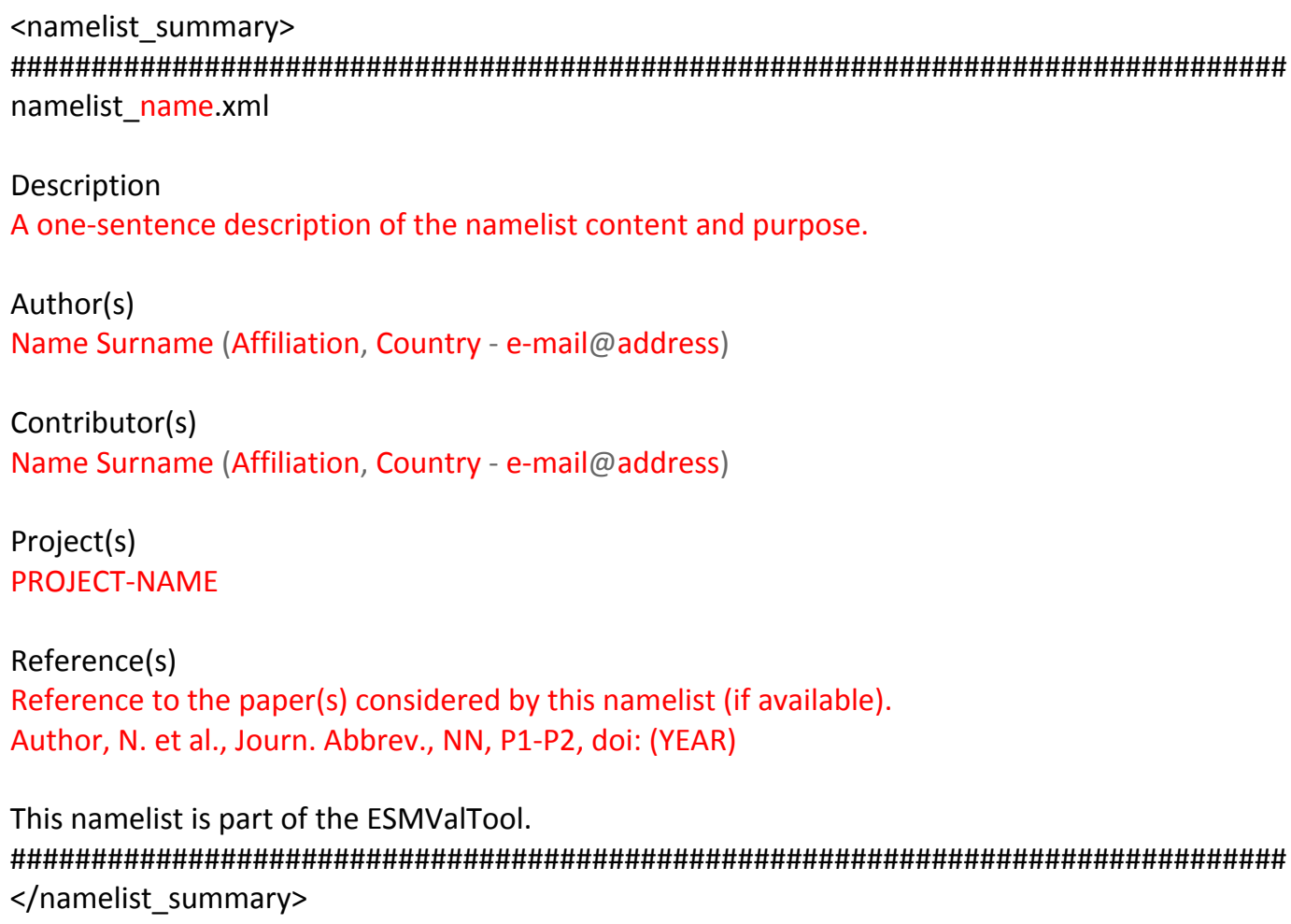




\subsection{Example namelist}

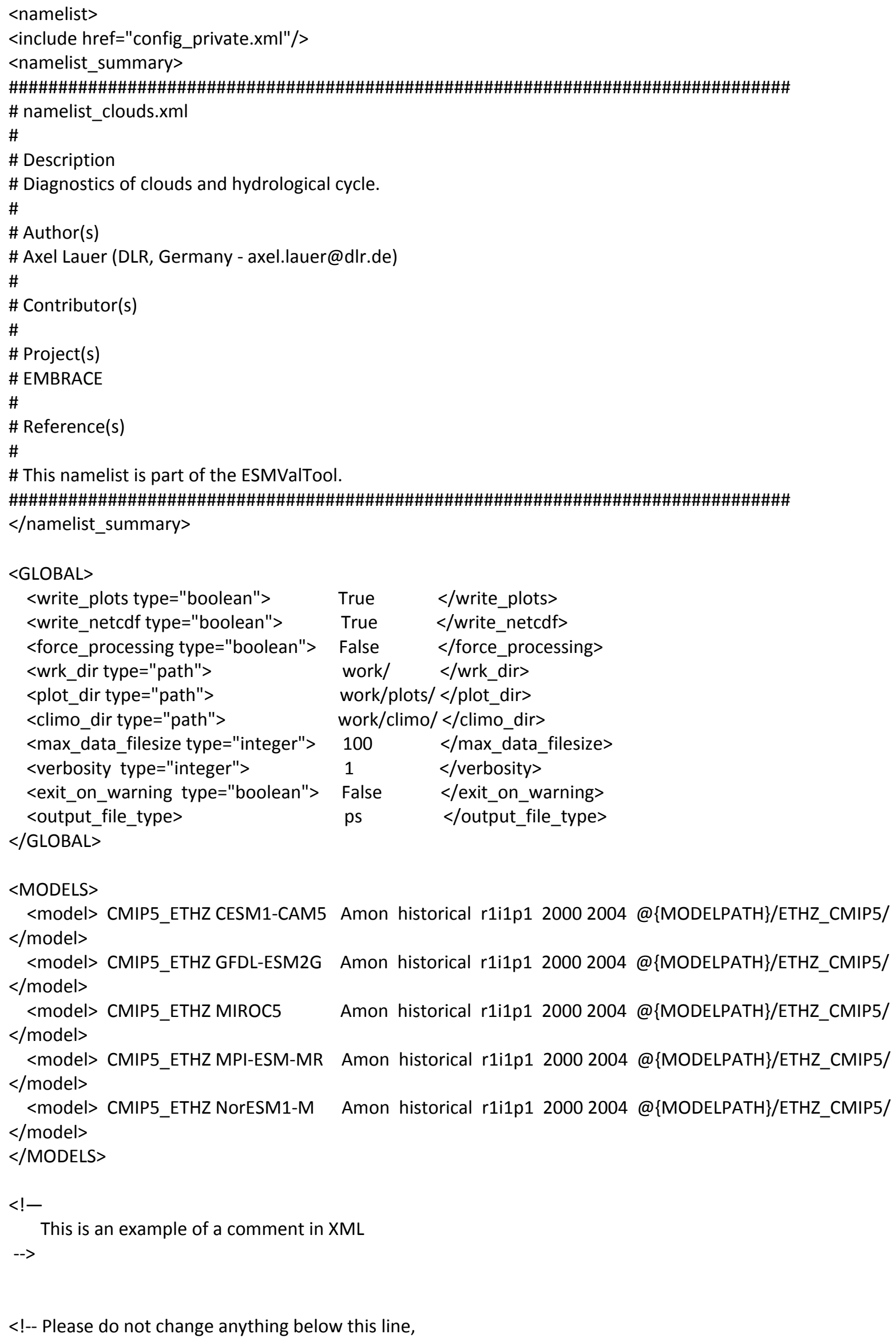




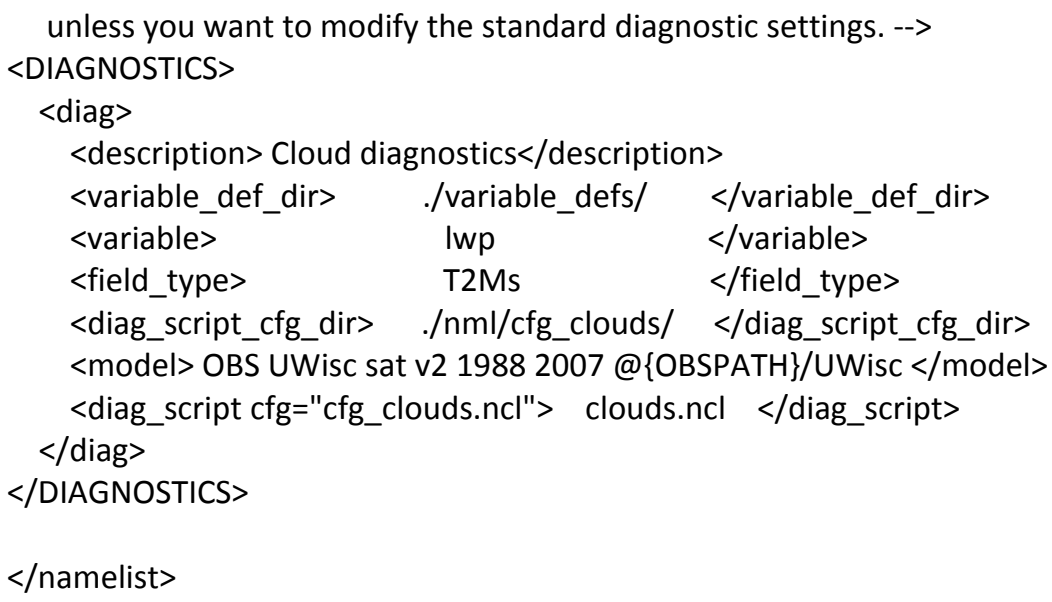

\section{Directory structure of the ESMValTool}

An overview of the directory structure used in the ESMValTool is given in Table S10 (section 11 - Annex A). This section summarizes the underlying principles of the structure.

- Common namelist settings (e.g., models, year ranges, diagnostics) are usually stored in one place.

- Less common settings may be hidden deeper in the directory structure (see also section 5).

- Diagnostic scripts that can be used by namelist entries are also stored in one place and it generally possible to combine them in a modular way (e.g., using the output of one routine as input for another).

- Reuse of code is strongly encouraged, i.e., one place for each functionality (modularity on the technical level).

- The goal is to centralize functionality in individual functions/procedures whenever there is the possibility of reusability. New developers are encouraged to consider building on or extending existing routines before introducing new ones.

- Routines are sorted into folders according to their functionality. Whenever possible, the hierarchy level of routines is reflected by their position in the directory structure.

\section{Configuration files $5.1 \mathrm{nml} / \mathrm{cfg} \_$diag/cfg_diag*.typ}

Diagnostic-specific settings are passed via configuration files. These are collected in the nml directory under subdirectories named like the corresponding diagnostic (e.g., cfg_aerosol/, cfg_perfmetrics/). The suffix ".typ" specifies the language the routine is written in.

There might be more than one configuration script per diagnostic set. All $\mathrm{cfg}_{-} *$ files for a diagnostic set need to be in the same folder specified by the < diag_script_cfg_dir $>$ entry of the namelist ( $\left.\mathrm{nml} / \mathrm{namelist}{ }_{-}^{*} . \mathrm{xml}\right)$ (see section 3.3 and Table S5).

The configuration settings are specified as attributes of the variable "diag_script_info" (in NCL via "diag_script_info@attribute = ...", see example below). In order to activate these attributes, "diag_script_info" must be set to "True". 


\section{Example (NCL)}

diag_script_info = True

diag_script_info@projection = "CylindricalEquidistant" ; map projection, e.g., Mollweide, Mercator diag_script_info@styleset = "CMIP5" ; "CMIP5", "DEFAULT"

diag_script_info@colormap = "WhiteBlueGreenYellowRed" ; e.g., WhiteBlueGreenYellowRed, rainbow

diag_script_info@ncdf = "default" ; enable to output to netCDF; either use "default" or give a full file name

\section{Example (Python)}

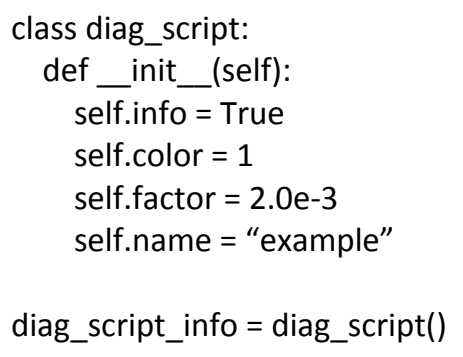

\section{Example (R)}

diag_script_info<-new()

diag_script_info[["begin_ref_year"]]<-1970

diag_script_info[["end_ref_year"]]<-2000

diag_script_info[["timescale"]]<-3

diag_script_info[["seasons"]]<-c("ann", "djf", "mam", "jja", "son")

\section{Running the ESMValTool}

The following section gives a brief description of the steps required by a user to run an existing diagnostic. As an example, the toy diagnostic MyDiag is chosen to illustrate the basic steps:

1. Check/edit the "namelist configuration file" (e.g., config_private.xml) (see section 3.4). If needed, set/change the base path names for the input data (model and observations) and the output data. Note: the use of a "namelist configuration file" is optional in order to allow for machine spacific standard search paths for input (and output) data without having to change the actual namelists. Alternatively, explicit path names can be used in the namelists.

2. Check/edit the main namelist $\mathrm{nml} /$ namelist_MyDiag.xml:

a. Set/check the file name of the "namelist configuration file" (defining the base path names) to be used by the ESMValTool (typically, this is the second line in the namelist, e.g., <include href="./config_private.xml"/>).

b. If needed, set the pathnames in the $<$ GLOBAL $>$ section for the "work" directory (wrk_dir), the directory for the plots (plot_dir) and the directory for reformatted files (climo_dir). See section 3.1 for details and Table S1 for a complete list of variables in the $<$ GLOBAL $>$ section.

c. In the $<$ MODELS $>$ section, define the model(s) to be used, including the root path for the actual model data, e.g., CMIP5_ETHZ MPI-ESM-LR Amon historical r1i1p1 2000 $2004 @\{M O D E L P A T H\} / E T H Z \_C M I P 5 /$ (see step 1 and section 3.4 for details on how to set base path names such as $@\{$ MODELPATH $\}$; alternatively, explicit path names can be 
used). See section 3.2, Table S2 and Table S3 for details. The first year (here: 2000) and last year (here: 2004) of the model data processed for each model is specified in this section.

d. Optionally, change variable and the field type in the $<$ DIAGNOSTICS $>$ section. See section 0 , Table S5 and Table S6 for details. An overview of the available "field types" is given in Table S7, Table S8 lists the available variables. Please note that the diagnostic section may include additional models and/or observational data.

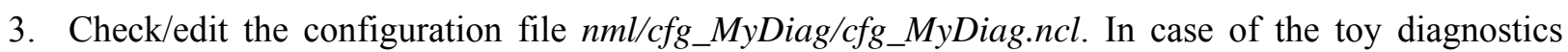
MyDiag, you can for example change the map projection for the contour plot by changing the value of the attribute diag_script_info@projection.

4. Run the ESMValTool (in the ESMValTool root directory): python main.py nml/namelist_MyDiag.nml

5. The output will be written to a subdirectory named like the diagnostics package (e.g., MyDiag) in the directories specified in the $<$ GLOBAL $>$ section of the namelist (see step 1 and also section 3.4). The default directories are: work/MyDiag for the NetCDF output and work/plots/MyDiag for the plot(s) (see also Figure 3). Acknowledgements and references are written to the file work/refsacknows_MyDiag.txt.

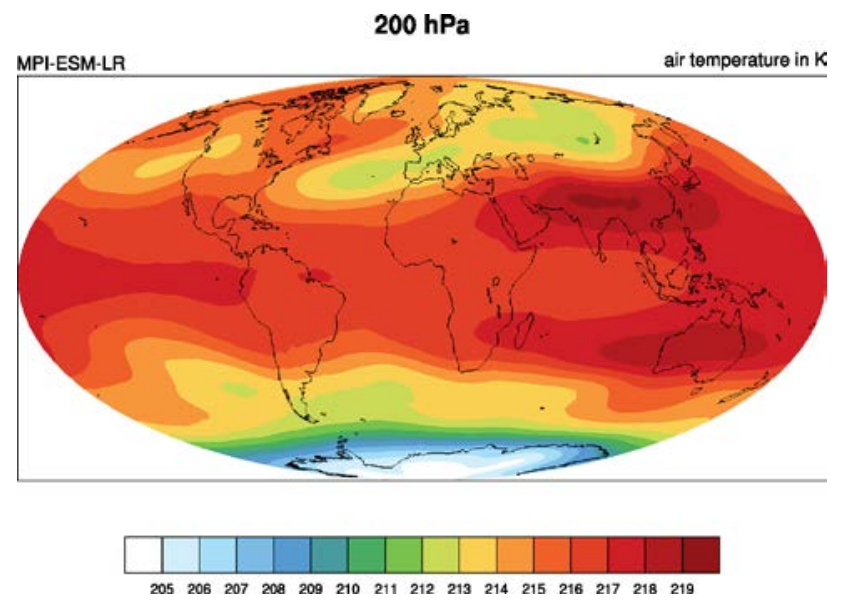

Figure 3 Example plot created by the toy diagnostic MyDiag showing the 5-year annual mean temperature at $200 \mathrm{hPa}$ from the CMIP5 historical run (r1ilp1) with the MPI-ESM-LR model.

\subsection{Available diagnostics and metrics}

An introduction to the available diagnostics and metrics packages implemented into the ESMValTool v1.0 including a description of the user settings, observational data used, references, and example plots is given in section 13 - Annex C.

\subsection{Model and observational data}

\subsubsection{Model data}

The project specifier (see Table S2) used in the $<$ MODELS $>$ section of the namelist (see section 3.2 for details) determines the directory structure and file naming convention expected by the ESMValTool. The two most commonly used project specifiers are CMIP5 and CMIP5_ETHZ. Both are used to process CMIP5 
data available from the Earth System Federation Grid (e.g., http://esgf.llnl.gov/). In order to download CMIP5 data, registration and creation of an "openID" is required. Instructions can be found here:

\section{http://cmip-pcmdi.1lnl.gov/cmip5/data_getting_started.html}

Besides downloading files individually, the CMIP5 data portal is capable of generation a script for automated download of model data using GNU wget (https://www.gnu.org/software/wget/). The wget script generation is recommended for downloading a large number files and/or large data volume.

Any CMIP5 files downloaded to a single directory can be moved to a CMIP5 like directory structure using the NCL script util/CMIP5_sort/CMIP5_sort.ncl. The script CMIP5_sort.ncl expects all files to be moved to the CMIP5 like directory structure in the current directory (“.”). The root directory for creating the CMIP5 like directory structure is specified in the script CMIP5_sort.ncl via the variable "outpath". The script is run via:

$$
\text { ncl <path of the ESMValTool>/util/CMIP5_sort/CMIP5_sort.ncl }
$$

The CMIP5 files will be moved into the directories outpath/experiment/mip/variable/name/ensemble/ for direct usage with the project specifier CMIP5_ETHZ (see below). "experiment", "mip", "variable" and "name" (= model name) are automatically extracted from the filename.

\section{The project specifier CMIP5}

Syntax of the CMIP5 specifier in the $<$ model $>$-tag (see section 3.2 and Table S2 for details):

$<$ model $>$ CMIP5 name mip experiment ensemble start_year end_year path $</$ model $>$

The project specifier CMIP5 will search for files in "path" with filenames matching the pattern

$$
\text { variable_mip_name_experiment_ensemble_*.nc }
$$

Note: "variable" is specified in the $<$ diag $>$-section (see section 3.3 for details). If "variable" is a derived quantity, all variables needed to calculate the derived quantity are processed automatically.

\section{The project specifier CMIP5_ETHZ}

Syntax of the CMIP5_ETHZ specifier in the $<$ model $>$-tag (see section 3.2 and Table S2 for details):

$<$ model>CMIP5_ETHZ name mip experiment ensemble start_year end_year path </model $>$

The project specifier CMIP5_ETHZ will search for files in

"path/experiment/mip/variable/name/ensemble" with filenames matching the pattern

variable_mip_name_experiment_ensemble_*.nc

This directory structure resembles the ESGF CMIP5 directory structure.

Note: "variable" is specified in the <diag>-section (see section 3.3 for details). If "variable" is a derived quantity, all variables needed to calculate the derived quantity are processed automatically.

\subsubsection{Observational / reanalysis data}

When possible, observations from the obs4MIPs/ana4MIPs archives are used in the model evaluation. These data are freely available from the ESGF in the same format as the CMIP simulations and can be directly used in the ESMValTool using the obs4mips or ana4mips project specifiers (see Table S2) in the namelist. 
A collection of all observational data used by the diagnostics of the ESMValTool (trunk) is hosted at DLR and can be made available (restrictions by the data owner permitting) on request (see Table S9). The reformatted observational data can be read using e.g., the OBS class in the namelist (see below).

All observations are tiered as follows:

- Tier 1: data sets from the obs4MIPs and ana4MIPs archives

- Tier 2: other freely available data sets

- Tier 3: restricted data sets (e.g., license agreement required)

Observational data sets not available in the obs4MIPs/ana4MIPs archives need to be reformatted according to the $\mathrm{CF} / \mathrm{CMOR}$ standard before they can be used (see sections 6.2.3 and 8.2 for more details).

\section{The project specifier $\mathrm{OBS}$}

Syntax of the OBS specifier in the $<$ model $>$-tag (see section 3.2 and Table $\mathrm{S} 2$ for details):

$<$ model $>$ OBS name case_name ensemble start_year end_year path $</$ model $>$

The project specifier OBS will search for files in "path" with filenames matching the pattern

OBS_name_casename_ensemble_fieldtype_variable*.nc

Note: "variable" and "fieldtype" are specified in the < diag>-section (see section 3.3 for details). If "variable" is a derived quantity, all variables needed to calculate the derived quantity are processed automatically.

\section{The project specifier obs4mips}

Syntax of the obs4mips specifier in the $<$ model $>$-tag (see section 3.2 and Table $\mathrm{S} 2$ for details):

$<$ model $>$ obs4mips name process_level ensemble start_year end_year path $<$ /model $>$

The project specifier obs4mips will search for files in "path/name/" with filenames matching the pattern

$$
\text { variable_name_processlevel_ensemble_*.nc }
$$

Note: "variable" is specified in the $<\operatorname{diag}>$-section (see section 3.3 for details). If "variable" is a derived quantity, all variables needed to calculate the derived quantity are processed automatically.

\subsubsection{Downloading and creating observational data sets}

obs4MIPS and ana4MIPs data sets ("tier 1", see above) are freely available from the ESGF. These data sets can be used directly with the ESMValTool without the need for reformatting. Examples of such data sets include:

- AIRS

- CERES-EBAF

- CFSR

- CloudSat-L3

- GPCP-1DD, GPCP-SG

- IFS-Cy31r2

- ISCCP

- MERRA

- MISR 
- MODIS

- TES

- TRMM, TRMM-L3

For the required file naming conventions and the expected directory structure, see section 6.2.2.

For all other (non-obs4MIPs and non-ana4MIPs) data sets, reformatting routines are provided with downloading and processing instructions in the header of the scripts. These reformatting scripts can be found in:

\section{reformat_scripts/obs/}

These reformat scripts can be specified in a namelist-file (e.g. namelist_reformat_obs.xml) and executed by calling the main.py script with the option "-r" or "--reformat":

$$
\text { python main.py }- \text { r namelist_reformat_obs.xml }
$$

This reformat namelist file contains the tag <REFORMAT> that can hold multiple <reformat_script>-tags specifying the reformat scripts to be called:

$<$ REFORMAT>

$<$ reformat_script $>$ /PATH/TO/REFORMATSCRIPT </reformat_script $>$

$</$ REFORMAT $>$

An example reformat namelist file is listed below:

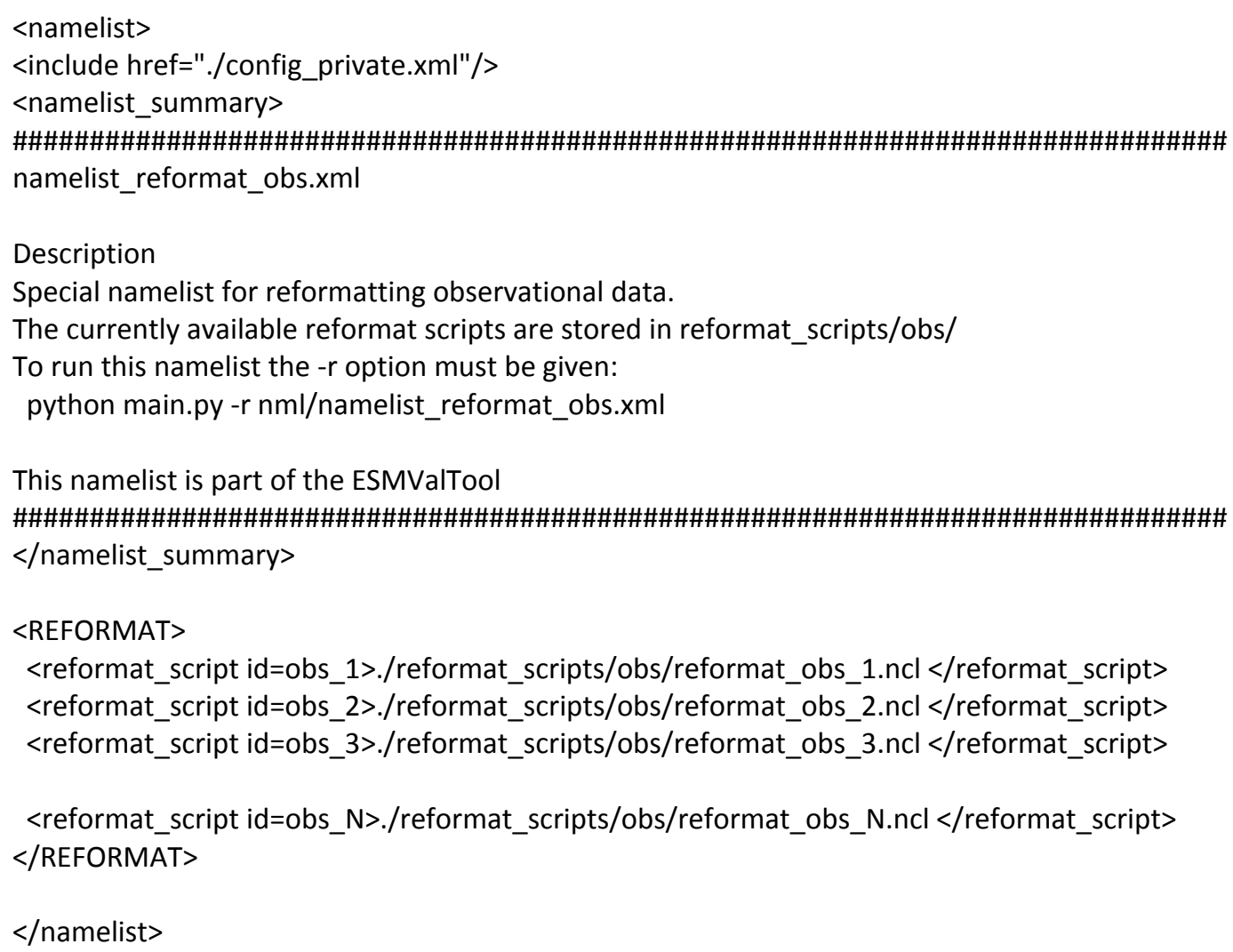

A list of available data sets and their corresponding reformatting routines are given in Table S9. 
Table S9 Observational data with available reformatting routines for use with the ESMValTool. See headers of the reformatting routines for downloading and processing instructions.

\begin{tabular}{|c|c|c|c|c|c|c|}
\hline Name & Tier & Description & Variables & Type & Time range & Script name \\
\hline ACCESS & 3 & $\begin{array}{l}\text { Aerosol vertical } \\
\text { profiles }\end{array}$ & $\mathrm{mmrbc}$ & Campaign & -- & reformat_obs_ACCESS.ncl \\
\hline AERONET & 2 & $\begin{array}{l}\text { Aerosol optical } \\
\text { depth at } 550 \mathrm{~nm}\end{array}$ & od550aer & Ground & 1992-2012 & reformat_obs_AERONET.ncl \\
\hline $\begin{array}{l}\text { AURA-MLS- } \\
\text { OMI }\end{array}$ & 2 & $\begin{array}{l}\text { Tropospheric } \\
\text { column ozone }\end{array}$ & tropoz & Satellite & $2005-2013$ & reformat_obs_AURA-MLS-OMI.ncl \\
\hline AURA-TES & 2 & Ozone mixing ratio & vmro3 & Satellite & 2005-2009 & reformat_obs_AURA-TES.ncl \\
\hline CARSNET & 2 & $\begin{array}{l}\text { Aerosol optical } \\
\text { depth at } 550 \mathrm{~nm}\end{array}$ & od550aer & Ground & $2002-1013$ & reformat_obs_CARSNET.ncl \\
\hline CASTNET & 2 & $\begin{array}{l}\text { Aerosol surface } \\
\text { level concentrations }\end{array}$ & $\begin{array}{l}\text { concso4, } \\
\text { concno3, } \\
\text { concnh4 }\end{array}$ & Ground & $1987-2012$ & reformat_obs_CASTNET.ncl \\
\hline CERES & 3 & $\begin{array}{l}\text { CERES synoptic data } \\
\text { (radiative fluxes at } \\
\text { surface, toa) }\end{array}$ & $\begin{array}{l}\text { rsuscs, rsus, } \\
\text { rsdscs, rsds, } \\
\text { rluscs, rlus, } \\
\text { rldscs, rlds, } \\
\text { rsutcs, rsut, } \\
\text { rlutcs, rlut }\end{array}$ & Satellite & 2004 & $\begin{array}{l}\text { reformat_obs_ceres-synoptic- } \\
\text { sfc.bash, reformat_obs_ceres- } \\
\text { synoptic-toa.bash }\end{array}$ \\
\hline CIRRUS & 3 & $\begin{array}{l}\text { Aerosol vertical } \\
\text { profiles }\end{array}$ & $\begin{array}{c}\text { mmrbc, } \\
\text { mmrbcfree }\end{array}$ & Campaign & $\begin{array}{l}\text { late Nov. } \\
2006\end{array}$ & reformat_obs_CIRRUS.ncl \\
\hline CloudSat & 1 & Cloud cover & clt & Satellite & 2006-2010 & reformat_obs_cloudsat.bash \\
\hline CMAP & 2 & Precipitation & $\mathrm{pr}$ & $\begin{array}{l}\text { merged } \\
\text { analysis }\end{array}$ & 1980-2013 & reformat_obs_CMAP.ncl \\
\hline CONCERT & 3 & $\begin{array}{l}\text { Aerosol vertical } \\
\text { profiles }\end{array}$ & $\begin{array}{c}\text { mmrbc, } \\
\text { conccnSTP1 } \\
4\end{array}$ & Campaign & - & reformat_obs_CONCERT.ncl \\
\hline CR-AVE & 3 & $\begin{array}{l}\text { Aerosol vertical } \\
\text { profiles }\end{array}$ & $\mathrm{mmrbc}$ & Campaign & - & reformat_obs_CR-AVE.ncl \\
\hline CRU & 3 & $\begin{array}{l}\text { Surface } \\
\text { temperature, } \\
\text { precipitation }\end{array}$ & tas, pr & Reanalysis & 1901-2006 & reformat_obs_CRU.ncl \\
\hline DC3 & 3 & $\begin{array}{l}\text { Aerosol vertical } \\
\text { profiles }\end{array}$ & $\mathrm{mmrbc}$ & Campaign & - & Reformat_obs_DC3.ncl \\
\hline Dong08-ARGO & 2 & $\begin{array}{l}\text { Derived ocean } \\
\text { mixed layer depth }\end{array}$ & mlotst & Campaign & 2001-2006 & reformat_obs_Dong08.ncl \\
\hline EANET & 2 & $\begin{array}{l}\text { Aerosol surface } \\
\text { level concentrations }\end{array}$ & $\begin{array}{l}\text { concso4, } \\
\text { concno3, } \\
\text { concnh4 }\end{array}$ & Ground & 2001-2005 & reformat_obs_EANET.csh \\
\hline EMEP & 2 & Aerosol surface & $\begin{array}{l}\text { concso4, } \\
\text { concno3, }\end{array}$ & Ground & 1970-2012 & reformat_obs_EMEP.csh \\
\hline
\end{tabular}




\begin{tabular}{|c|c|c|c|c|c|c|}
\hline & & level concentrations & $\begin{array}{l}\text { concnh4, } \\
\text { concpm2p5, } \\
\text { concpm10 }\end{array}$ & & & \\
\hline Emmons & 2 & $\begin{array}{l}\text { Vertical profiles of } \\
\text { gases }\end{array}$ & various & Campaign & variable & reformat_obs_Emmons.csh \\
\hline ERA-Interim & 3 & $\begin{array}{l}\text { Basic climate } \\
\text { parameters }\end{array}$ & $\begin{array}{l}\text { ta, ua, va, } \\
\text { zg, hus, tas, } \\
\text { tos, ps, psl, } \\
\text { tauu, tauv, } \\
\text { clwvi, clivi, } \\
\text { sftlf }\end{array}$ & Reanalysis & 1979-2012 & reformat_obs_ERA-Interim.ncl \\
\hline $\begin{array}{l}\text { ERA-Interim } \\
\text { precipitation }\end{array}$ & 3 & Precipitation & $\mathrm{pr}$ & Forecast & 1979-2014 & $\begin{array}{l}\text { reformat_obs_ERA- } \\
\text { Interim_precip.ncl }\end{array}$ \\
\hline $\begin{array}{l}\text { ERA-Interim } \\
\text { fluxes }\end{array}$ & 3 & $\begin{array}{l}\text { Basic climate } \\
\text { parameters, surface } \\
\text { fluxes }\end{array}$ & $\begin{array}{l}\text { pr, evspsbl, } \\
\text { hfls, hfss, } \\
\text { rsns, rlns }\end{array}$ & Forecast & $2000-2005$ & $\begin{array}{l}\text { reformat_obs_ERA-Interim- } \\
\text { surffluxes.ncl }\end{array}$ \\
\hline $\begin{array}{l}\text { ESACCI- } \\
\text { AEROSOL }\end{array}$ & 2 & $\begin{array}{l}\text { Aerosol optical } \\
\text { depth at } 550 \mathrm{~nm}\end{array}$ & od550aer & Satellite & $1997-2011$ & reformat_obs_ESACCI-AEROSOL.ncl \\
\hline ESRL & 2 & $\begin{array}{l}\mathrm{CO}_{2} \text { surface level } \\
\text { concentrations }\end{array}$ & $\mathrm{co} 2$ & Ground & $1973-2012$ & reformat_obs_ESRL.ncl \\
\hline ETH-SOM-FFN & 2 & $\mathrm{pCO}_{2}$ ocean surface & spco2 & & $1998-2011$ & reformat_obs_ETH-SOM-FFN.csh \\
\hline GCP & 2 & $\begin{array}{l}\text { Carbon budget } \\
\text { parameters }\end{array}$ & co2flux & & 1959-2011 & reformat_obs_GCP.ncl \\
\hline GLOBALVIEW & & $\begin{array}{l}\text { CO surface level } \\
\text { concentrations }\end{array}$ & vmrco & Ground & 1991-2008 & reformat_obs_GLOBALVIEW.ncl \\
\hline GPCC & 2 & Precipitation & $\mathrm{pr}$ & Reanalysis & $1901-2010$ & reformat_obs_GPCC.ncl \\
\hline GTO-ECV & & Total column ozone & toz & Satellite & $1996-2010$ & reformat_obs_GTO-ECV.ncl \\
\hline HadCRUT & 2 & $\begin{array}{l}\text { Near-surface air } \\
\text { temperature }\end{array}$ & tas & Ground & $1850-2013$ & reformat_obs_HadCRUT.ncl \\
\hline HadISST & 2 & $\begin{array}{l}\text { Sea ice } \\
\text { concentrations and } \\
\text { sea surface } \\
\text { temperatures }\end{array}$ & sic, ts & Reanalysis & 1870-2014 & reformat_obs_HadISST \\
\hline HALOE & 2 & $\begin{array}{l}\text { Water vapor mixing } \\
\text { ratio }\end{array}$ & vmrh2o & Satellite & 1991-2002 & reformat_obs_HALOE.ncl \\
\hline HIPPO & 3 & $\begin{array}{l}\text { Aerosol vertical } \\
\text { profiles }\end{array}$ & $\mathrm{mmrbc}$ & Campaign & -- & reformat_obs_HIPPO.ncl \\
\hline IMPROVE & 2 & $\begin{array}{l}\text { Aerosol surface } \\
\text { level concentrations }\end{array}$ & $\begin{array}{c}\text { concso4, } \\
\text { concno3, } \\
\text { concnh4, } \\
\text { concbc, } \\
\text { concoa, } \\
\text { concpm2p5, } \\
\text { concpm10 }\end{array}$ & Ground & 1988-2011 & reformat_obs_IMPROVE.ncl \\
\hline
\end{tabular}




\begin{tabular}{|c|c|c|c|c|c|c|}
\hline INCA & 3 & $\begin{array}{l}\text { Aerosol vertical } \\
\text { profiles }\end{array}$ & $\begin{array}{c}\text { conccnSTP5, } \\
\text { conccnSTP1 } \\
4, \\
\text { conccnSTP1 } \\
4\end{array}$ & Campaign & -- & reformat_obs_INCA.ncl \\
\hline LACE & 2 & $\begin{array}{l}\text { Aerosol size } \\
\text { distributions }\end{array}$ & sizecn & Campaign & -- & reformat_obs_LACE.ncl \\
\hline LandFlux-EVAL & 3 & Evapotranspiration & et, et-sd & $\begin{array}{l}\text { Synthesis } \\
\text { product } \\
\text { (model + } \\
\text { observati } \\
\text { ons) }\end{array}$ & 1989-2005 & reformat_obs_landflux-eval.ncl \\
\hline Melpitz & 2 & $\begin{array}{l}\text { Aerosol size } \\
\text { distributions }\end{array}$ & sizecn & Campaign & -- & reformat_obs_Melpitz.ncl \\
\hline MERRA & 1,2 & $\begin{array}{l}\text { Precipitation, } \\
\text { climate parameters }\end{array}$ & $\mathrm{pr}$ & Reanalysis & 1979-2011 & reformat_obs_MERRA.bash \\
\hline MODIS-CFMIP & 2 & Ice water path & clivi & Satellite & 2003-2014 & reformat_obs_MODIS-CFMIP.ncl \\
\hline MODIS_L3_C6 & 2 & $\begin{array}{l}\text { Ice water path, } \\
\text { liquid water path, } \\
\text { total cloud cover }\end{array}$ & $\begin{array}{l}\text { clivi, clwvi, } \\
\text { clt }\end{array}$ & Satellite & $2003-2014$ & reformat_obs_modis.ncl \\
\hline NCEP & 2 & $\begin{array}{l}\text { Essential climate } \\
\text { variables }\end{array}$ & $\begin{array}{l}\text { ta, ua, va, } \\
\text { zg, hus, tas }\end{array}$ & Reanalysis & $1948-2012$ & $\begin{array}{l}\text { reformat_obs_NCEP.ncl, } \\
\text { reformat_obs_NCEP_daily.ncl }\end{array}$ \\
\hline NIWA & 3 & Total column ozone & toz & Reanalysis & $1980-2010$ & reformat_obs_NIWA.ncl \\
\hline $\begin{array}{l}\text { NOAA } \\
\text { interpolated } \\
\text { OLD }\end{array}$ & 2 & $\begin{array}{l}\text { Interpolated } \\
\text { outgoing longwave } \\
\text { radiation }\end{array}$ & rlut & Satellite & $1975-2013$ & $\begin{array}{l}\text { reformat_obs_NOAA-PSD-Interp- } \\
\text { rlut-ncl }\end{array}$ \\
\hline NSIDC & & $\begin{array}{l}\text { Sea ice } \\
\text { concentrations }\end{array}$ & sic & Satellite & $1978-2010$ & reformat_obs_NSIDC.ncl \\
\hline OAFlux & 2 & Latent heat flux & hfls & Reanalysis & $1958-2013$ & reformat_obs_OAFlux.ncl \\
\hline Putaud & 2 & $\begin{array}{l}\text { Aerosol size } \\
\text { distributions }\end{array}$ & sizecn & Campaign & -- & reformat_obs_Putaud.ncl \\
\hline SALTRACE & 3 & $\begin{array}{l}\text { Aerosol vertical } \\
\text { profiles }\end{array}$ & $\mathrm{mmrbc}$ & Campaign & -- & reformat_obs_SALTRACE.ncl \\
\hline SeaWIFS & 2 & $\begin{array}{l}\text { Ocean } \\
\text { biogeochemistry }\end{array}$ & chl & & $1997-2010$ & reformat_obs_SeaWIFS.csh \\
\hline SOCAT & 2 & Ocean surface $\mathrm{CO}_{2}$ & spco2 & & $1970-2011$ & reformat_obs_SOCAT.csh \\
\hline SRB & 2 & Radiative fluxes & $\begin{array}{l}\text { rsut, rlut, } \\
\text { rlutcs }\end{array}$ & Satellite & $1983-2007$ & reformat_obs_SRB.ncl \\
\hline takahashi14 & 2 & $\begin{array}{l}\text { Ocean } \\
\text { biogeochemistry }\end{array}$ & talk & & 2005 & reformat_obs_takahashi14.csh \\
\hline TC4 & 3 & $\begin{array}{l}\text { Aerosol vertical } \\
\text { profiles }\end{array}$ & $\mathrm{mmrbc}$ & Campaign & -- & reformat_obs_TC4.ncl \\
\hline
\end{tabular}




\begin{tabular}{|c|c|c|c|c|c|c|}
\hline Texas & 3 & $\begin{array}{l}\text { Aerosol vertical } \\
\text { profiles }\end{array}$ & $\begin{array}{c}\text { mmraer, } \\
\text { mmrbc }\end{array}$ & Campaign & -- & reformat_obs_Texas.ncl \\
\hline Tilmes & & Ozone mixing ratios & vmro3 & In-situ & $1995-2009$ & reformat_obs_Tilmes.ncl \\
\hline TRMM_3B42 & 2 & Precipitation & $\mathrm{pr}$ & Satellite & 1998-2014 & $\begin{array}{l}\text { reformat_obs_TRMM_3B42_daily. } \\
\text { ncl, } \\
\text { reformat_obs_TRMM_3B42_3hourl } \\
\text { y.ncl }\end{array}$ \\
\hline UCN-Pacific & 3 & $\begin{array}{l}\text { Aerosol vertical } \\
\text { profiles }\end{array}$ & conccnSTP3 & Campaign & -- & reformat_obs_UCN-Pacific.ncl \\
\hline UWisc & 3 & Liquid water path & clwvi & Satellite & $1988-2007$ & reformat_obs_UWisc.ncl \\
\hline WOA09 & 2 & $\begin{array}{l}\text { Climatological } \\
\text { ocean fields }\end{array}$ & $\begin{array}{l}\text { so, sos, to, } \\
\text { tos }\end{array}$ & $\begin{array}{l}\text { Analyzed } \\
\text { climatolog } \\
\text { y }\end{array}$ & -- & reformat_obs_WOA09.ncl \\
\hline woa2005 & 2 & $\begin{array}{l}\text { Ocean } \\
\text { biogeochemistry }\end{array}$ & 02 & & 2005 & reformat_obs_woa2005.csh \\
\hline
\end{tabular}

\subsection{The acknowledgements log file}

Each diagnostics in the tool automatically generates a log file containing a list of authors/contributors, details on the projects to be acknowledged and the reference papers to be cited. It also provides a list of the used model and observational data with the corresponding references.

The $\log$ is created automatically when running the ESMValTool. The log file is named refsacknow_<diagnostics $>$.txt and written to the directory defined in the $<$ GLOBAL $>$ section of the namelist (variable wrk_dir, see section 3.1), e.g., work/refs-acknows_MyDiag.txt (see also section 6, step 4).

An example excerpt of an acknowledgements log file is provided below.

\section{Example}

+++++++++++++ ESMValTool REFERENCES and ACKNOWLEDGEMENTS LOG +++++++++++++

Namelist file: namelist_perfmetrics_CMIP5.xml

Creation date: Wed Dec 16 22:58:29 CET 2015

ESMValTool version: 0.9.1

Host name: \#\#\#

User name: \#\#\#

Licensed under the Apache License, Version 2.0 (the "License"); you may not use this file except in compliance with the License. You may obtain a copy of the License at

http://www.apache.org/licenses/LICENSE-2.0

Unless required by applicable law or agreed to in writing, software distributed under the License is distributed on an "AS IS"BASIS,

WITHOUT WARRANTIES OR CONDITIONS OF ANY KIND, either express or implied. 
See the License for the specific language governing permissions and limitations under the License.

Please acknowledge the use of the ESMValTool.

Please cite Eyring et al., ESMValTool (v1.0) - a community diagnostic and performance metrics tool for routine evaluation of Earth System Models in CMIP, Geosci. Model Dev., 2016.

For the specific diagnostics, see below.

$===$ perfmetrics_main. $\mathrm{ncl}===$

AUTHOR(S):

$-A-\# \#$

CONTRIBUTOR(S):

$-\mathrm{C}-\# \# \#$

$-\mathrm{C}-\# \# \#$

$-\mathrm{C}-\# \#$

REFERENCE(S) FOR THIS DIAGNOSTIC:

-R- Please cite Righi et al., Geosci. Model Dev., 8, 733-768 doi:10.5194/gmd-8-733-2015, 2015.

-R- Please cite Gleckler et al., J. Geophys. Res., 113, D06104, doi:10.1029/2007JD008972, 2008.

REFERENCE(S) FOR THE OBSERVATIONS:

-R- NCEP - Kalnay et al., Bull. Amer. Meteor. Soc., 77, 437-470, 1996.

-R- ERA-Interim

-R-AIRS

-R- CERES-EBAF

$-\mathrm{R}-\mathrm{SRB}$

ACKNOWLEDGEMENTS FOR THE PROJECTS:

-P- EU FP7 project EMBRACE

-P- DLR project ESMVal

PREPROCESSING/REFORMATTING (ESMValTool v0.9.1):

Variable: ta

Model: ERA-Interim

Input file(s):

(1) OBS_reanaly_ERA-Interim_1_T3M_ta_2000-2001.nc

Original source file(s) of all input file(s):

-S- (1) @\{OBSPATH\}/Tier3/ERA-Interim/OBS_ERA-Interim_reanaly_1_T3M_ta.nc

Fixes applied to original source file(s): none

Reference(s) of original source file(s):

(1) Dee, D. P. et al., Q. J. Roy. Meteor. Soc., 137, 553-597, doi:10.1002/qj.828, 2011.

Model: MPI-ESM-LR

Input file(s):

(1) CMIP5_ETHZ_Amon_historical_MPI-ESM-LR_r1i1p1_T3M_ta_1998-2002.nc

Original source file(s) of all input file(s):

-S- (1) @\{MODELPATH\}/ETHZ_CMIP5/historical/Amon/ta/MPI-ESM-LR/r1i1p1/ta_Amon_MPI-ESM-

LR_historical_r1i1p1_199001-199912.nc (tracking_id: ea695cd3-6234-4ddf-a68e-b4d82a2e7305)

-S- (2) @\{MODELPATH\}/ETHZ_CMIP5/historical/Amon/ta/MPI-ESM-LR/r1i1p1/ta_Amon_MPI-ESM-

LR_historical_r1i1p1_200001-200512.nc (tracking_id: f9134520-0445-4461-9a48-14d8663dab74)

Fixes applied to original source file(s): ./reformat_scripts/fixes/CMIP5_MPI-ESM-LR_fix.ncl

$[\ldots]$ 


\section{Part II: Developer's Guide}

\section{Writing a diagnostic script or a metrics set}

The development of a new diagnostic (or set of diagnostics) requires the following steps before getting started:

- Creating a development branch in the applicable project subdirectory of the subversion repository (via svn, see section 12.1). Developers are encouraged to work actively through the subversion repository. Regular "commits" to the repository help to document changes introduced to the ESMValTool and allow for efficient sharing of code with other developers.

- Setting up a wiki documentation page for the new diagnostic/performance metrics following the template on the ESMValTool wiki (see section 12.3).

- Creating a standard namelist following the template on the ESMValTool wiki (see also section 3.5).

- If needed, opening an issue on the Mantis bug tracker (see section 12.2).

General coding rules and conventions:

- Regular updates of the development branch (svn merge, see also section 12) are strongly recommended in order to keep it synchronized with the trunk.

- Modularizing all diagnostic scripts as much as possible, using the general-purpose code in lib/ and separating the diagnostic calculations from the plotting routines.

- Before creating new functions or procedures, it should be considered to use or extend the existing routines within lib/. Each header (see section 7.1) provides an overview of the already implemented functions and procedures.

- Functions and procedures specific to a given diagnostic shall go in the subdirectory diag_scripts/aux/<diagnostic $>$ (see Table S10).

- Main namelist, diag_scripts, functions and procedures shall be documented within the respective file using the templates provided on the ESMValTool wiki (see also sections 3.5, 7.1 and 12.3).

- Each diag_script shall contain a call to the function write_reference (see also section 7.7) in order to generate a respective acknowledgements log file (section 7.7).

The reintegration of the development branch into the trunk can only be done by the core development team (see section 9) who shall be contacted as soon as the branch is ready for integration into the trunk. Before contacting the core development team the following items should be checked:

- The new branch runs works with different configuration options.

- If the lib/routines have been modified, all the diagnostics using these routines have to be tested (see automated testing, section 7.9).

- The new code complies with the coding rules and standards (see section 7.5) and follows the ESMValTool directory structure (see Table S10).

- All authors, contributors and data are properly acknowledged and referenced in the acknowledgements log file (see section 7.7).

- If the new observational data are used, the scripts to "cmorize" these data shall also be made available and placed as reformat_obs_<name $>$ into the folder reformat_scripts/obs/. Once the branch has been merged to the trunk, it shall be moved to the branches/legacy/subfolder. 


\subsection{Standard template}

All (diagnostic) scripts and namelists in the ESMValTool are documented following the standards defined by templates (see section 3.5 for the namelist template). The following describes the standard header for diagnostics scripts. The parts in red are the ones to be modified by the author.

- The modification history is in reverse chronological order (i.e., most recent on top) and the last entry always contains the "written" statement (optionally with a statement such as "based on" if derived from existing code).

- The author of each entry in the modification history is indicated with the author id as given in the author list in the master reference file (doc/MASTER_authors-refs-acknow.txt, e.g., A_surn_na = surname, name).

- All lines should be limited to a maximum of 79 characters (see section 7.5). Exceptions can be made to improve the readability of the code.

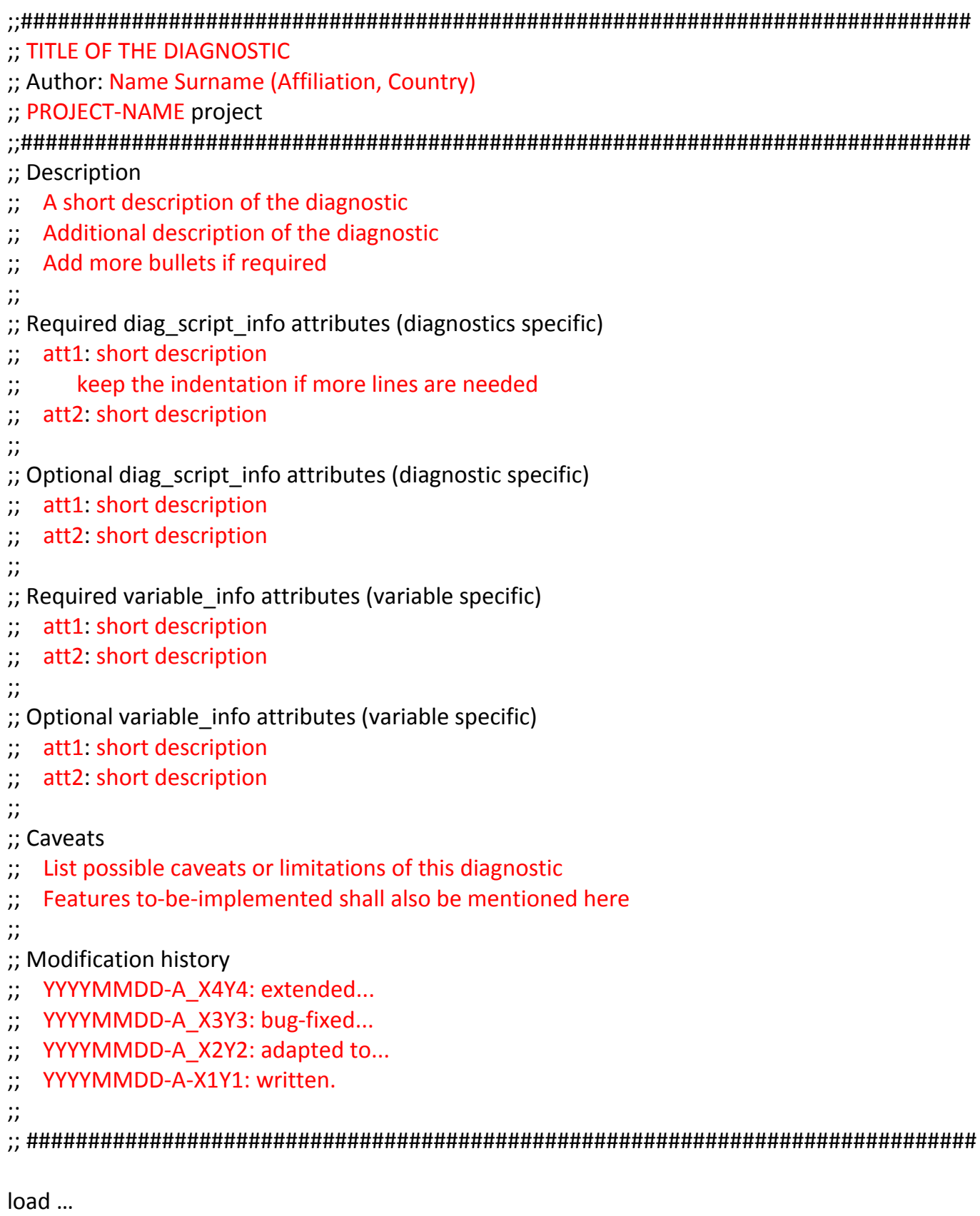


load ...

begin

$\cdots$

end

\subsection{Library functions}

The folder diag_scripts/lib/ contains general purpose routines used by several diagnostic scripts, these library routines are grouped in subfolders by language, i.e.,

$$
\begin{gathered}
\text { diag_scripts/lib/ncl } \\
\text { diag_scripts/lib/python } \\
\text { diag_scripts/lib/R }
\end{gathered}
$$

Library routines are grouped into individual files by topic, some examples for the NCL library routines are:

- diag_scripts/lib/ncl/latlon.ncl: routines to compute grid cell areas, weighted area averages, etc...

- diag_scripts/lib/ncl/regridding.ncl: routines interfacing the ESMF regridding functions in NCL

- diag_scripts/lib/ncl/statistics.ncl: statistical routines not (yet) implemented in the standard distribution of NCL

- diag_scripts/lib/ncl/style.ncl: centralized control of NCL plot styles, e.g., defines line colors/dashes/thickness for each model name in CMIP5, based on the style files in diag_scripts/lib/ncl/styles/.

For further details on the library functions, see the project wiki or the documentation given in the header of the functions themselves (see section 7.1 for a template).

\subsection{Plotting functions}

The folder plot_scripts/ contains general purpose routines used for plotting by the diagnostic scripts. The plotting functions should facilitate the separation of computing the diagnostic and displaying the result. To this end they should handle both the case when called directly from the diagnostic script (with data to visualize as an argument), and the case when the computed diagnostic is passed along as a netCDF file. These plotting routines are grouped in subfolders by language,

- $\quad$ lot_scripts/ncl

- plot_scripts/python

- $\quad$ plot_scripts/R

Each subfolder further groups the plotting routines into files by topic, e.g., for the NCL library routines:

- plot_scripts/ncl/contour_maps.ncl: interfaces NCL plotting routines for contour map plots, contour polar maps and adding markers to contour maps 
- plot_scripts/nc/scatterplot.ncl: interfaces NCL plotting routines for of scatter plots

For further details on the plotting functions, see the project wiki (section 12.3) or the inline documentation in the functions themselves.

\subsection{Adding new variables}

Adding new variables requires changes to reformat_scripts/recognized_vars.dat (section 7.4.1) and possibly also to reformat_scripts/recognized_units.dat (section 7.4.2). In addition, a new definition file variable_defs/<varname $>$.ncl is needed (section 7.4.3; see Table S8 for a list of currently available variable definition scripts). If the variable is a non-derived variable (explained in section 7.4.3) it also needs to be defined in a file named reformat_scripts/cmor/CMOR_<variable $>$.dat (see section 7.4.4)

\subsection{1 reformat_scripts/recognized_vars.dat}

New variables have to be added to reformat_scripts/recognized_vars.dat. Two lines are added per variable:

- $\quad$ std name $=$ varname standard CMOR variable name

- alt_name $=$ alternative name 1 , alternative name $2, \ldots$ comma separated list of alternative variable names

\section{Example (surface pressure)}

- $\quad$ std_name $=$ ps

- $\quad$ alt_name $=$ aps,PS,psurf

The ESMValTool reformat scripts will look for variable "varname" in the input files. If not found, the alternative variable names "alternative name 1", "alternative name 2", etc. are tried before an error message is issued that the variable could not be found.

\subsection{2 reformat_scripts/recognized_units.dat}

The file reformat_scripts/recognized_units.dat contains a list of known units. If needed, the unit of the newly added variable can be added. There are two lines per unit:

- $\quad$ std_name $=$ unit

standard CMOR unit

- $\quad$ alt_name $=$ alternative unit

comma separated list of possible alternative units and corresponding conversion factor, defined as units[cmor] $=$ units[alternative $] *$ factor

\section{Example (dobson units)}

- $\quad$ std unit $=$ DU

- alt_unit $=\mathrm{g} \mathrm{m}-2,4.6707 \mathrm{e}-5, \mathrm{~kg} \mathrm{~m}-2, \mathrm{~mol} \mathrm{~m}-2,2.2414 \mathrm{e}-3$

\subsection{3 variable_defs/varname.ncl}

The file variable_defs/<varname $>$.ncl is a NCL script containing the declaration of the variable "varname" including its specific attributes. In case of derived variables, a function "calculate" calculating the derived variable must be defined in the script <varname>.ncl (see Table S8 for a list of currently available variable definition scripts). 


\section{Remarks}

1. For derived variables, a statement specifying the (standard, non-derived) variables required to calculate the derived variable is needed. In the example given below, this statement in the beginning of the NCL script looks like

; Requires: rsut:T2*s,rsutcs:T2*s

In this example, the two standard variables "rsut" and "rsutcs" are needed to calculate the shortwave cloud forcing.

2. Variable attributes are specified as attributes of the variable "variable_info" (see examples below). In order to activate the variable attributes, "variable_info" must be set to "True". Some examples for frequently used attributes are:

- variable_info@derived=False (True)

- variable_info@long_name=“...”

- variable_info@units=“...”

- variable_info@standard_name=“...”

- variable_info@short_name ="..."

\section{Example (precipitation, standard variable)}

; Requires: none

variable_info $=$ True

variable_info@derived=False

\section{Example (shortwave cloud forcing, derived variable)}

; Requires: rsut:T2*s,rsutcs:T2*s

$[\ldots]$

variable_info $=$ True

variable_info@derived=True

variable_info@long_name = "CS Shortwave cloud radiation effect"

variable_info@units = "W m-2"

undef("calculate")

function calculate(index [1] : integer, variable [1] : string, field_type [1] : string)

;i return_val [1] : logical

;; Arguments:

i; index - index to current infile defined in the 'interface_data/ncl.interface'-file

i; variable - Current variable as string

;i field_type - string with field type classification

;; Return value:

;i data_new - logical

local tmp, tmp1, tmp2, dum1, dum2, dum, i, verbosity

begin

data_new $=$ True

tmp1 = read_data(index, "rsut", "T2Ms")

tmp2 = read_data(index, "rsutcs", "T2Ms")

dum1 = extract_data(index, tmp1, -1, 0, 0)

dum2 = extract_data(index, tmp2, -1, 0, 0)

dum $=$ dum 1

dum $=$ dum2 - dum 1 


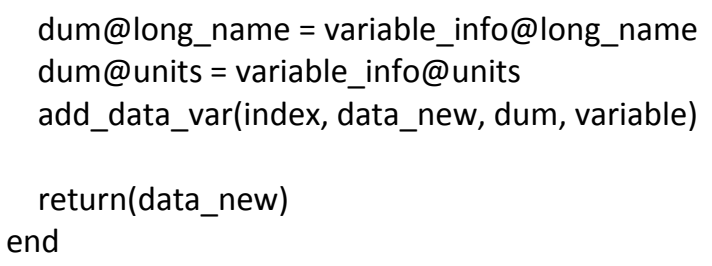

\subsection{4 reformat_scripts/cmor/CMOR_variable.dat}

Each standard variable (non-derived) also needs a configuration file indicating the expected units of the variable. The expected units are read from the file reformat_scripts/cmor/CMOR_variable.dat which follows the definitions in the official CMOR tables for CMIP5. If this file is missing for a specific variable, it can be downloaded from http://pcmdi.github.io/cmor-site/tables.html. If a CMOR table for the new variable is not available, the user can create a new one based on the existing tables (e.g., following the example in reformat_scripts/cmor/CMOR_mmrbcfree.dat based on reformat_scripts/cmor/CMOR_mmrbc.dat).

\section{Example, reformat_scripts/cmor/CMOR_pr.dat}

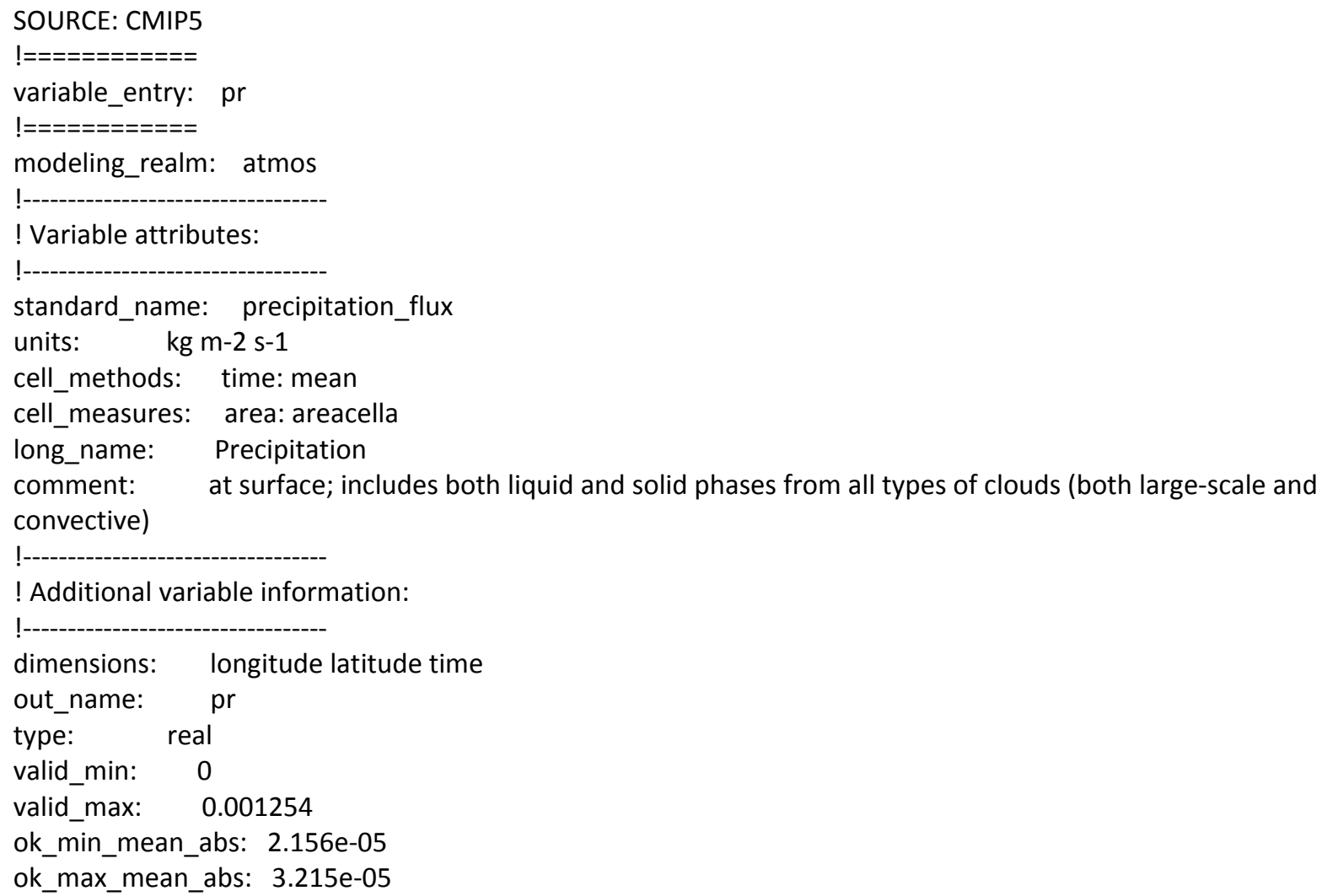

\subsection{Coding rules and standards}

The purpose of the code conventions used in ESMValTool is to ensure a high degree of consistency in the code layout. Consistently structured code increases readability and understanding of the code making it easier for developers and users work with a given piece of the code base. It is important to emphasize two points:

- Checking the code consistency should be done by software as this allows the check to be done automatically. 
- Code checkers are available at util/ncl-checker/pep8.py (NCL) and util/pep8-checker/pep8.py (Python).

The code conventions are guidelines and should be treated as such. There are circumstances when it is advisable, for various reasons such as improved readability, to ignore some of the guidelines.

\section{Code conventions used for Python}

Python code should conform to the PEP-8 style guide [PEP8 2001]. Recommended tools to check Python code is the official PEP8-checker (util/pep8-checker/pep8.py) and PyFlakes. Further information on usage of these tools can be found on the ESMValTool wiki pages.

\section{Code conventions for NCL}

NCL code in ESMValTool should follow the PEP-8 style guides. An NCL adapted version of the Python PEP-8 checker is available in the ESMValTool repository (util/ncl-checker/pep8.py). See the ESMValTool wiki (section 12.3) for further details. Please note that the NCL checker may report some false-positive (e.g., the reading symbol -> is not recognized as such).

\section{Code conventions for $\mathbf{R}$}

The code conventions for $\mathrm{R}$ should conform to the formatting produced by the $\mathrm{R}$ parser tree. See the ESMValTool wiki (section 12.3) for further details.

\subsection{Documentation of software}

In order to ensure that all code can be maintained, all diagnostic packages must be well documented. It is the responsibility of the software developers to embed their documentation into the code and to provide a summary of their diagnostics on the ESMValTool wiki (see also section 7.8). Documentation systems exist to organize embedded documentation into well structured, linked documents.

- R: documentation should follow CRAN guidance.

- Python: the Sphinx package allows embedded documentation to be assembled into indexed web pages (see section 7.8)

- NCL and namelists: a Sphinx extension has been developed to extract code documentation for NCL and namelists (see section 7.8)

\subsection{The acknowledgements log file}

The acknowledgements log file automatically created by each diagnostic (see also section 6.1 ) is written by the function write_references (interface_scripts/messaging.ncl, see below), which uses the tags defined in the master reference/acknowledgements file (doc/MASTER_authors-refs-acknow.txt) as input. This master file lists all authors and contributors (tags starting with A_), the diagnostic references (tags with D_), references for observational data (tags $\left.E_{-}\right)$and projects (tags $\left.\mathrm{P}_{-}\right)$.

\section{The function write_references}

The function write_references (defined in interface_scripts/messaging.ncl) should be called at the end of each diagnostic script in order to write the acknowledgements $\log$ file (section 7.7). The function has the arguments "author(s)", "contributors", "diagnostics", "observations", "projects" which are arrays of strings. 
All strings ("tags") used must be defined in the master reference file doc/MASTER_authors-refs-acknow.txt. The tags are then replaced by the function write_references with their definition when writing the acknowledgements log file. All tags in the master reference file are sorted by category of which there are four in total:

- A_xxx $=$ authors, contributors $(\mathrm{xxx}=$ author name $)$

e.g., A_\#\#\#

- D_xxx $=$ diagnostics

e.g., D_gleckler08jgr $=$ Gleckler et al. (2008)

- $\quad$ E_xxx $=$ observational data

e.g., E_era40 = ERA40

- $\quad P_{-} \mathrm{xxx}=$ project

e.g., P_embrace $=$ EU FP7 project EMBRACE

write_references(diag_script, \

"A_\#\#\#", I

(/"D_righi15gmd", "D_gleckler08jgr"/), ।

(/"E_kalnay96bams", "E_erainterim", "E_airs", "E_ceresebaf", "E_srb"/), \

(/"P_embrace", "P_esmval"/))

\subsection{Documentation of source code}

The Sphinx documentation generator (http://sphinx-doc.org) is used to organize and format ESMValTool documentation, including text which has been extracted from source code. Sphinx can help to create documentation in a variety of formats, including HTML, LaTeX (and hence printable PDF), manual pages and plain text.

Sphinx may be obtained from http://sphinx-doc.org/install.html; an overview of its workings is available at http://sphinx-doc.org/tutorial.html. In ESMValTool, Sphinx has been used to set up the files in doc/sphinx. Running make <target $>$ in that directory will cause the documentation to be built, and its output placed in the build/<target $>$ subdirectory. Here, $<$ target $>$ is the format required - for example, html, latexpdf, man or text for the four example formats mentioned above. Running make by itself will generate a complete list of output formats.

Sphinx was originally developed for documenting Python code, and one of its features is that it is able using the so-called autodoc extension - to extract documentation strings from Python source files and use them in the documentation it generates. This feature apparently does not exist for NCL source files (such as those which are used in ESMValTool), but it has been mimicked (or - more-or-less - reverse-engineered) here via the Python script doc/sphinx/scripts/process_ncl_docs.py, which walks through a subset of the ESMValTool NCL scripts, extracts function names, argument lists and descriptions (from the comments immediately following the function definition), and assembles them in a subdirectory of doc/sphinx/source. These output files are in the so-called reStructuredText format (see, e.g., http://docutils.sourceforge.net/rst.html), which is the markup language used by Sphinx; running make in doc/sphinx builds the ESMValTool documentation from them, as noted above. 


\subsection{Automated testing}

Any changes to a programming code have the risk of introducing unwanted side effects on some other parts of a code and introduce bugs. Routine and automated testing is therefore essential to maximize the code quality and ensure integrity of all diagnostics implemented within ESMValTool.

\subsubsection{Setup and general workflow}

Automated testing within the ESMValTool is implemented on two complementary levels:

- unittests are used to verify that small code units (e.g. functions/subroutines) provide the expected results

- integration testing is used to verify that a diagnostic integrates well into the ESMValTool framework and that a diagnostic provides expected results. This is verified by comparison of the results against a set of reference data generated during the implementation of the diagnostic.

\section{Installation of the test environment}

All scripts required to run the test environment are provided together with the ESMValTool code. Two external python packages are required which can be installed using the python package manager (pip; https://pypi.python.org/pypi/pip) as follows in a linux environment:

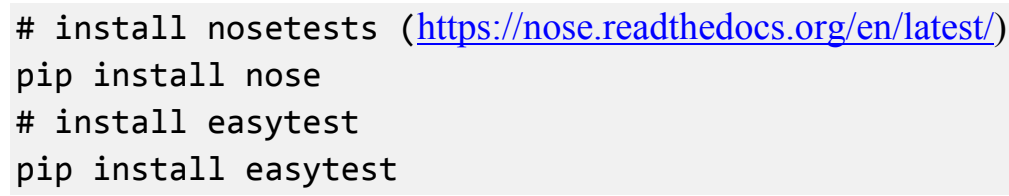

\section{General functionality of testing framework}

Each diagnostic is expected to produce a set of well-defined results. These are files in a variety of formats and types (e.g. graphics, data files, ASCII files ...). While testing results of a diagnostic, a special namelist file is executed by ESMValTool which runs a diagnostic on a limited set of test data only. A small test data set is chosen to minimize executing time for testing while ensuring on the other hand that the diagnostic produces the correct results. The following general tests are implemented at the moment for diagnostics with available test data:

- Check for file availability: a check is performed that all required output data have been successfully generated by the diagnostic. A missing file is always an indicator for a failure of the program.

- File checksum: While the previous test only checks if a file is available, the checksum verifies if the content of a file is similar. Currently the MD5 checksum is used to verify that contents of a file are the same. The MD5 checksum is a good proxy for the similarity of two files and is used regularly to ensure integrity between files when transferring files between different computers.

- Graphics check: For graphic files an additional test is therefore implemented which verifies that two graphical outputs are identical. This is in particular useful to verify that outputs of a diagnostic remain the same after code changes.

\section{Testing the ESMValTool diagnostics}

Unittests are implemented for each diagnostic independently. Details on running unittests using nose is as simple as going to the ESMValTool root directory and then execute the following shell command: 
\# run nosetests

nosetests

This will search recursively for test files and execute these tests. A statistic on success and failures is provided at the end of execution. More details on using nose can be found in the package's documentation (https://nose.readthedocs.org/en/latest/).

To run integration tests for each diagnostic, a small script needs to be written once. An example for a file named esmvaltooltest.py is provided in section 7.9.2. To run all tests for diagnostics implemented in this file the following command needs to be executed:

\# run integration tests

python esmvaltooltest.py

A summary of success and failures is provided as output.

\subsubsection{Example test implementation for a diagnostic}

In the following an example is given how to implement a test environment for a new diagnostic with just a few lines of code.

File: esmvaltooltest.py

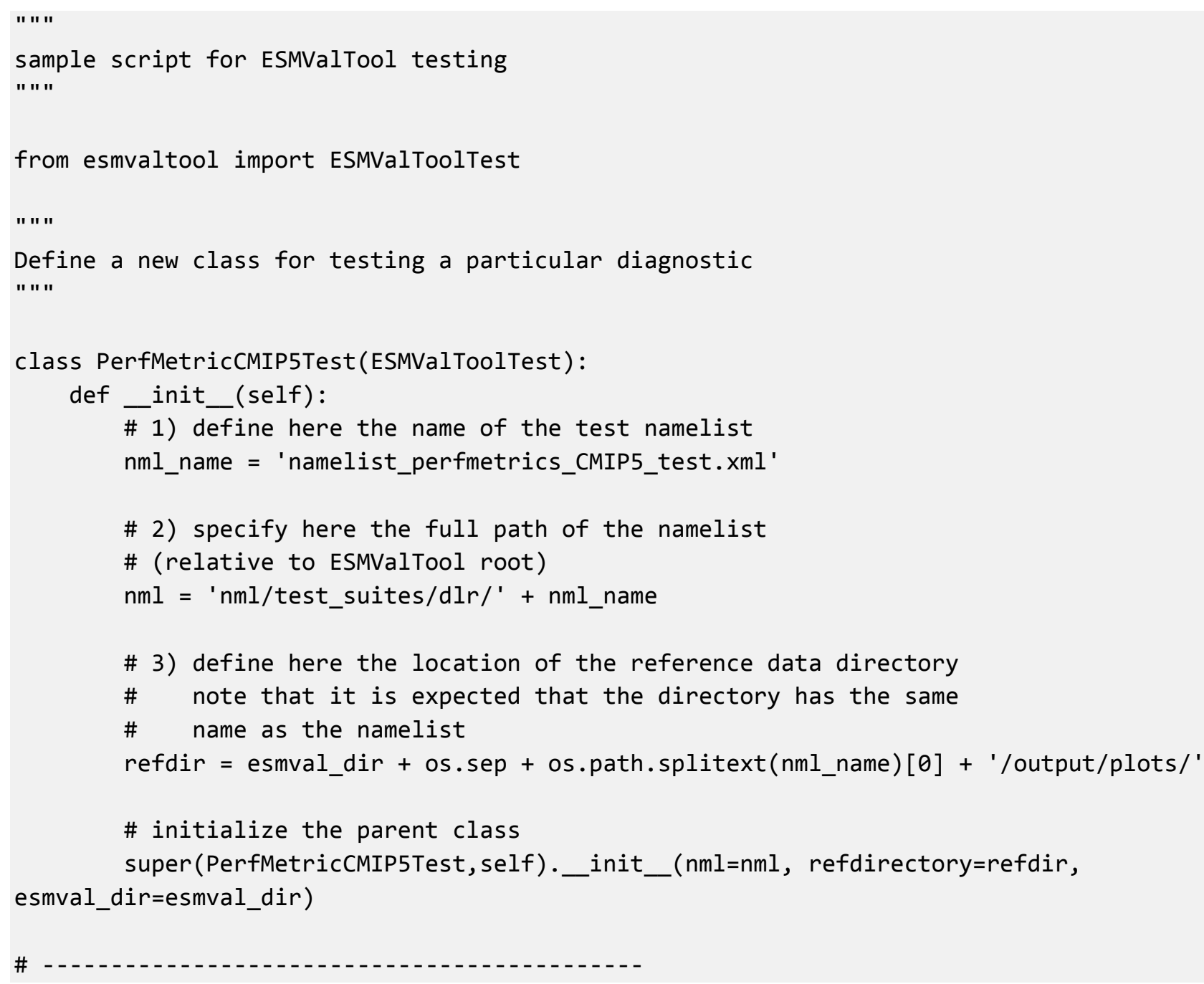


\# This is how you run a test

PT $=$ PerfMetricCMIP5Test () \# create instance of test class

PT.run_nml() \# run the testing namelist

PT.run_tests (execute=False, graphics=None, checksum_files='all',files='all') \#

perform tests

\section{Scientific documentation of a diagnostic script or metrics set}

An important part of the implementation of a new diagnostic script is the documentation of the diagnostic on the ESMValTool wiki as well as the documentation of the observational data sets used. The former should comply with the standard template for new diagnostics (see section 8.1 below) and the latter should include instructions how to download the observational data and, if necessary, scripts to convert it to the format required in ESMValTool, see section 8.2 below.

\subsection{Standard template}

When implementing a new diagnostic script or metrics set, it should be documented on the ESMValTool wiki by creating a new wiki entry and filling out the below standard template:

\begin{tabular}{|c|c|}
\hline \multicolumn{2}{|c|}{ Title of diagnostic / performance metric set } \\
\hline Developers & first name surname 1 (DLR tag 1), first name surname 2 (DLR tag 2), etc. \\
\hline Contributors & first name surname $1(D L R \operatorname{tag} 1)$, first name surname $2(D L R$ tag 2$)$, etc. \\
\hline Date of documentation & yyyy-mm-dd \\
\hline $\begin{array}{l}\text { Name of standard namelist } \\
\text { (XyZ) }\end{array}$ & $\begin{array}{l}\text { For Xyz the following naming conventions is used: } \\
\text { For papers } \\
\text { XyZ=SurnameYearJournalabbreviation (e.g., stocker12jgr, stocker12sci1, stocker12sci2). } \\
\text { For copies of reports that are not publicly available: } \\
\text { XYZ=OrgYearTitleabbrev (e.g., unep10water, unep11gap, roysoc09geoengineering). } \\
\text { For grouped set of diagnostics and performance metrics that do not follow a published paper or } \\
\text { report } \\
\text { an intuitive name that describes the science theme (e.g., XyZ=aerosol, MyDiag, SAMonsoon, } \\
\text { Sealce) }\end{array}$ \\
\hline User settings & $\begin{array}{l}\text { list of all settings that have to be checked/changed by a user in order to run the diagnostic (e.g., } \\
\text { pathnames, configuration files, color tables, supported model names, etc.) }\end{array}$ \\
\hline Brief summary & $1-3$ sentence summary \\
\hline Status & Planned/work in progress/finished and merged to trunk \\
\hline $\begin{array}{l}\text { CMOR variable name (realm, } \\
\text { frequency, dimension) }\end{array}$ & e.g., tas (atmos, monthly mean, longitude latitude plevs time) \\
\hline Development branch & e.g., https://svn.dlr.de/ESM-Diagnostic/source/branches/CCMI/ \\
\hline Link to relevant Mantis issue & e.g., https://mantis.dlr.de/mantis/view.php?id=11742 \\
\hline 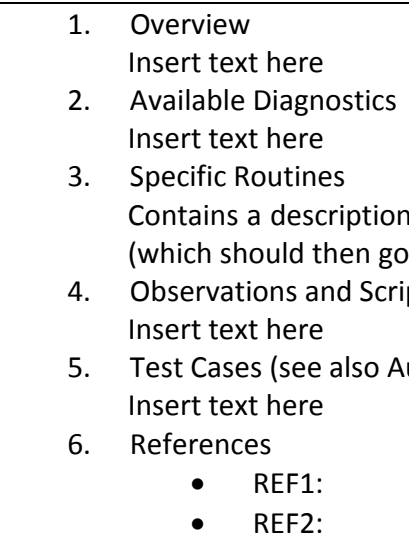 & $\begin{array}{l}\text { of specific routines being developed for the given diagnostic that helps to identify common code } \\
\text { n the lib/ } \\
\text { ts (also see Model and observational data below) } \\
\text { tomated testing on the project wiki and section } 7.9 \text { ) }\end{array}$ \\
\hline
\end{tabular}




\subsection{Model and observational data}

\subsubsection{Overview}

When possible, observations from the obs4MIPs/ana4MIPs archives are used in the model evaluation (see section 6.1). These data are freely available from the ESGF in the same format as the CMIP simulations and can be directly used in the ESMValTool using the obs4mips or ana4mips class in the namelist (see also section 6.2).

Observational data sets not available in these archives need to be reformatted according to the CF/CMOR standard before they can be used. In this case a reference to the official URL is provided such that a user can get the latest version of the data set as well as a description and a script how to convert the data set to the format required by the ESMValTool. These conversion scripts are collected in reformat_scripts/obs/reformat_obs_<NAME $>$.ncl. The reformatting routines must be documented with a standard header providing all information required to retrieve and process the data, as well as their availability (Tier 1, Tier 2, or Tier 3).

All observations are tiered as follows:

- Tier 1: data sets from the obs4MIPs and ana4MIPs archives

- Tier 2: other freely available data sets

- Tier 3: restricted data sets (e.g., license agreement required)

For Tier 2 and 3 data, the developer shall also provide links and helper scripts through the reformatting routines, following the template for the standard header described in section for the reformatting routines. An example can be found here:

$$
\text { reformat_scripts/obs/reformat_obs_AURA-MLS-OMI.ncl. }
$$

An overview on the available reformatting scripts for Tier 2 and 3 data is given in Table S9.

\subsubsection{Standard header for the reformatting routines for observational data}

This is a template of the standard header for the reformat_obs routines. The parts in red are the ones to be modified by the author. The modification history is given in reverse chronological order (i.e., most recent on top) and the last entry always contains the written statement. The author of each entry in the modification history shall be indicated with the author tag, as given in the master reference file (doc/MASTER_authorsrefs-acknow.txt), e.g., A_surn_na = surname, name. All lines should be limited to a maximum of 79 characters.

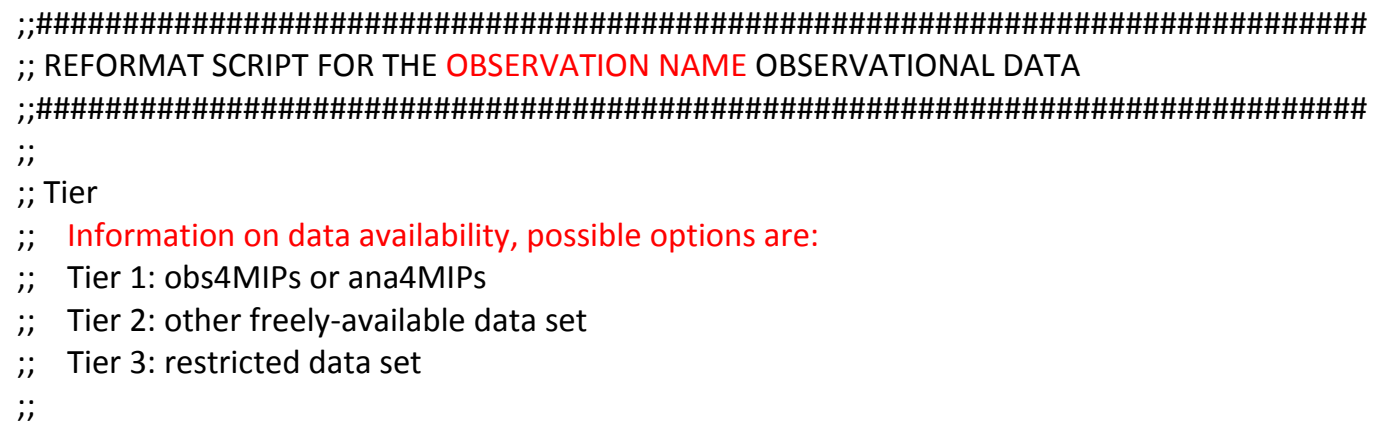




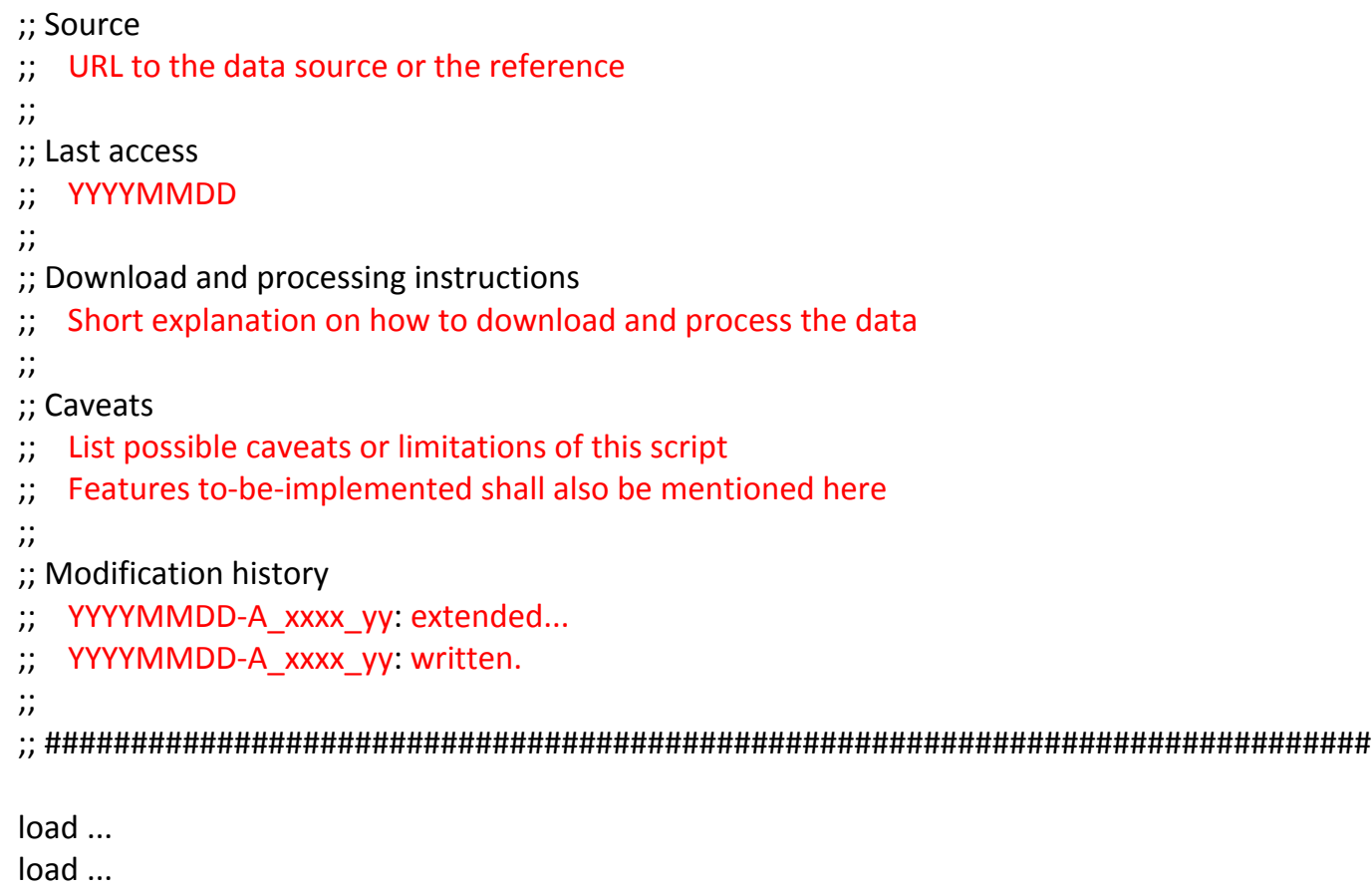

\section{The ESMValTool core development team \\ 9.1 Main Contacts}

Please do not hesitate to contact Veronika Eyring (veronika.eyring@dlr.de) or Axel Lauer (axel.lauer@dlr.de) for general or technical questions on the ESMValTool or in case you have problems with access to Mantis (see section 12.2), svn (section 12.1), wiki (section 12.3).

\subsection{Core Development Team}

- Veronika Eyring (DLR, Germany), veronika.eyring@dlr.de

ESMValTool Core PI and Developer: contact for requests to use the ESMValTool and for collaboration with the development team (all projects), and access to Mantis, svn, wiki

- Axel Lauer (DLR, Germany), axel.lauer@dlr.de

ESMValTool Core Developer: contact for technical questions (all projects), and access to Mantis, svn, wiki

- Mattia Righi (DLR, Germany), mattia.righi@dlr.de

ESMValTool Core Developer: contact for technical questions (all projects)

- Martin Evaldsson (SMHI, Sweden), martin.evaldsson@smhi.se

ESMValTool Core Developer EMBRACE: contact for general technical questions and technical implementation of SMHI led diagnostics (only for the EMBRACE project)

Contacts for specific diagnostic sets are the respective authors, as listed on the corresponding wiki pages and in the source code. 


\subsection{Merge requests}

\subsubsection{Workflow core development team}

The following workflow followed by the ESMValTool core development team takes place whenever a developer requests the merging of a diagnostics set into the trunk:

1. Check that the developer submits a standard namelist that calls a set of diagnostics / metrics

2. Check that the related documentation on the wiki is compliant with documentation templates for diagnostics and metrics sets (see section 7.1).

3. Check that the code follows coding rules and standard (see section 7.5).

4. Check that a namelist is provided for automated testing that runs on 2-3 models and a small set of observations/reference model/idealized data

- Verify that such a reduced and small set of observations/reference model/idealized data is delivered for each diagnostic that is called by the standard namelist

- Verify that an example plot + netCDF for automated testing created with this reduced data set is provided for each diagnostic that is called by the standard namelist as a reference

5. Check that also the full set of observations is provided that allows a sophisticated scientific application of the (full) standard namelist

6. Check that the observations are documented on the ESMValTool Wiki and that a reformat routine is available in case the original source is not in CMOR standard

7. Run the automated testing with all available diagnostics

8. Iterate with developer(s) on points 1-7 until the above items are fulfilled and the reference plots for all standard namelists included in the trunk can be reproduced

\subsubsection{Responsibilities of ESMValTool developers}

1. Accept the ESMValTool license agreement / terms of use.

2. Provide documentation on the wiki that is compliant with documentation templates for diagnostics and metrics sets.

3. Provide well documented code that follows the coding rules and standards.

4. For each merge request to implement a diagnostic set into the trunk.

Scientific analysis

- Provide the code for all diagnostics and metrics that are called.

- Standard namelist running on (if possible) all CMIP5 models and corresponding plots that are produced (for wiki).

- Provide the full set of observations that allows a sophisticated scientific application of the full standard namelist list (indicate source and if applicable license issues).

- Provide documentation for the observations on the wiki and a reformat routine if the original source does not follow the CMOR standard.

Automated testing (see section 7.9) 
- Provide the code for automated testing for the diagnostic set that should be integrated into the trunk.

- Provide a namelist for automated testing.

- Provide a reduced and small set of observations/reference model/idealized data for each diagnostic that is called by the testing namelist.

- Provide NetCDF + example plots for automated testing based on the reduced data set and the standard namelist as a reference.

5. Name a contact person providing (scientific) support for your diagnostics.

\section{References}

Flato, G., Marotzke, J., Abiodun, B., Braconnot, P., Chou, S. C., Collins, W., Cox, P., Driouech, F., Emori, S., Eyring, V., Forest, C., Gleckler, P., Guilyardi, E., Jakob, C., Kattsov, V., Reason, C., and Rummukainen, M.: Evaluation of Climate Models. In: Climate Change 2013: The Physical Science Basis. Contribution of Working Group I to the Fifth Assessment Report of the Intergovernmental Panel on Climate Change, Stocker, T. F., D. Qin, G.-K. Plattner, M. Tignor, S.K. Allen, J. Boschung, A. Nauels, Y. Xia, V. Bex and P.M. Midgley (Ed.), Cambridge University Press, Cambridge, United Kingdom and New York, NY, USA, 2013.

NCL (2014) The NCAR Command Language (Version 6.2.1) [Software]. (2014). Boulder, Colorado: UCAR/NCAR/CISL/VETS. http://dx.doi.org/10.5065/D6WD3XH5

PEP8 (2001) https://www.python.org/dev/peps/pep-0008/

Taylor, K. E., Stouffer, R. J., and Meehl, G. A.: An Overview of Cmip5 and the Experiment Design, B Am Meteorol Soc, 93, 485-498, 2012.

XML http://www.w3.org/TR/xml/www.gmail.com 


\section{Annex A - More tables}

Table S10 Directory structure of the ESMValTool sorted by file type.

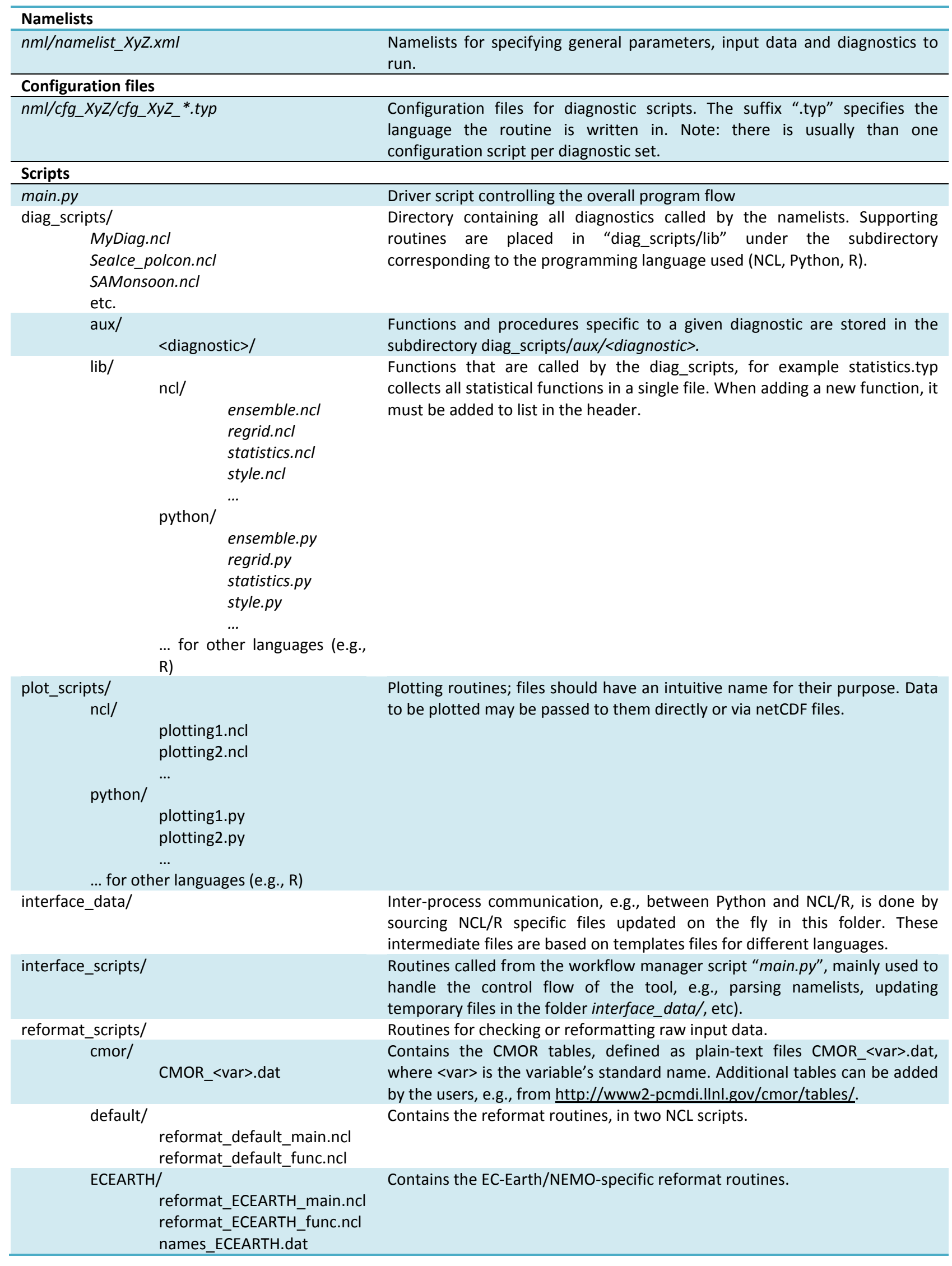




\begin{tabular}{|c|c|c|}
\hline \multicolumn{3}{|c|}{ make_Ism3d.sc } \\
\hline & $\begin{array}{l}\text { reformat_EMAC_main.ncl } \\
\text { reformat_EMAC_func.ncl } \\
\text { names_EMAC.dat }\end{array}$ & Contains the EMAC-specific reformat routines. \\
\hline & $<$ project>_<model>_fixes.ncl & $\begin{array}{l}\text { Contains the user-defined, project- and model-specific fixes, defined as NCL } \\
\text { scripts < project }>\text { _<model>_fixes.ncl. A template is also provided for the user } \\
\text { to add more fixes. }\end{array}$ \\
\hline & obs/ & Contains specific reformat routines for "cmorizing" observational data. \\
\hline & constants.ncl & $\begin{array}{l}\text { Contains general-purpose functions and procedure, called by the default, the } \\
\text { ECEARTH- and the EMAC-specific routines. }\end{array}$ \\
\hline & recognized_units.dat & $\begin{array}{l}\text { Provides a list of possible alternative units to the CMOR standard and the } \\
\text { corresponding conversion factor. Can be extended by the user. }\end{array}$ \\
\hline & recognized_vars.dat & $\begin{array}{l}\text { Provides a list of possible alternative variable names to the CMOR standard } \\
\text { names. Can be extended by the user. }\end{array}$ \\
\hline & variable_defs/ & $\begin{array}{l}\text { Declaration of variables, variable specific attributes and calculation of } \\
\text { derived variables }\end{array}$ \\
\hline \multicolumn{3}{|c|}{ Data folders } \\
\hline & & $\begin{array}{l}\text { The data folders are specified in } \mathrm{nml}_{\text {/namelist_* }}^{*} \text {, and thus may be different } \\
\text { from the defaults given here. These folders contain the output generated by } \\
\text { the ESMValTool and are created on the fly if needed. Note that these folders } \\
\text { do not need to be in the same directory as the source code. They can be } \\
\text { arbitrarily specified in the namelist as path relative to the root path. Using } \\
\text { symbolic links is another option to separate the actual data from the code. }\end{array}$ \\
\hline climo/ & & $\begin{array}{l}\text { Quality checked and derived netCDF files, reformatted from the original } \\
\text { data. }\end{array}$ \\
\hline plots/ & & Destination directory for graphics files. \\
\hline work/ & & $\begin{array}{l}\text { Miscellaneous files produced during run-time, e.g., optional netCDF output } \\
\text { and references/acknowledgements. }\end{array}$ \\
\hline
\end{tabular}

Table S11 Workflow of reformat routines.

\section{Control flow of reformat_default}

The reformat_default_main.ncl script sets the global variables as defined in reformat.py (input and output paths, variable name and field, model name and ensemble, etc.) and then performs a list of operations calling various functions and procedures defined in reformat_default_func.ncl. The workflow is as follows:

- $\quad$ find grid type: the data can be defined on a standard rectilinear grid or on an irregular grid. In the latter case, the script does not modify the grid properties and additionally attaches the area field (the area weights) for the irregular grid to the output file. The location of the area file is typically defined as an entry in the namelist, for example by using the project class CMIP5_gridfile where the final entry is the full path to the area file, see Table S2.

- read variable: the selected variable is read from the input file. If the variable is not found, the reading function checks for possible alternative variable names (as specified in recognized_vars.dat), before issuing an error message.

- apply project- and model-specific fixes: if a fixing procedure is found in the fixes/ directory for the selected project and model, it is called at this point in order to apply the user-defined corrections to the data.

- create time-series: the variable is read for the selected time range (start_year-end_year) and a time-series is created.

- $\quad$ rank/field consistency: the consistency of variable's rank with the given field (T3M, T2Mz, T2Ms, etc.). A simple calculation of the zonal mean is performed in case a rank 4 variable is provided with T2?z field.

- check fill values: a default missing values is assigned if the variable does not have one. The function then looks for data values that might represent undefined missing values. Currently it considers: -999., -9999., -99999., -999999., 1.e15, -1.e34. Finally, the ESMValTool default missing values (1.e20) is assigned as a standard_FillValue to the variable.

- reformat time coordinate: the time coordinate is reformatted according to the $\overline{C M O R}$ standard. If a calendar attribute is not assigned, the standard is used. The consistency of the time-series with the selected time range is checked.

- reformat vertical coordinate (applies only to certain fields and to rectilinear grids): the vertical coordinate is assigned "Pa" units, converting from the most common alternative units (mbar, bar, $\mathrm{hPa}$ ) if required. The ordering is set from top to bottom (monotonically decreasing).

- $\quad$ reformat latitude coordinate (applies only to certain fields and to rectilinear grids): the ordering is set from South to North (monotonically increasing).

- reformat longitude coordinate (applies only to certain fields and to rectilinear grids): the ordering is set from 0 to 360 degrees.

- $\quad$ check units: consistency of the variable's units with the CMOR standard is checked. The CMOR table for the selected 
variable must be available in the CMOR/ directory (an error message is issued otherwise). Units renaming and conversion can also be performed, if the corresponding information is given in recognized_units.dat.

- $\quad$ set variable attributes: the CMOR standard attributes are assigned to the selected variable. The corresponding CMOR table must be available in the CMOR/ directory (an error message is issued otherwise).

- $\quad$ write output file: the variable reformatted according to the CMOR standard is written in the selected output file.

- add info for irregular grids (applies only to irregular grids): the area file of the irregular grid is added, this file may later be used for averaging.

\section{Control flow of reformat_ECEARTH}

This reformat procedure can be used to convert raw EC-Earth/NEMO files to a format that complies with the ESMValTool requirements. It performs the following additional operations compared with the default workflow:

- find_name: translate the EC-Earth/NEMO name to a CMOR name using the table names_ECEARTH.dat.

- $\quad$ sub_staggergrid: determine grid type $(T, U, V)$ and add that information to the filename.

- mask_land: land points have the value 0 in the raw files, not a fill value (missing value). This routine sets land points (as in the landmask file) to fill values.

- rename_time: rename time variable from EC-Earth name to standard name and remove the attribute _FillValue.

- rename lev: vertical coordinate name in raw files depends on grid, rename it to lev. Requires the external input table names_ECEARTH.dat.

- add_ijcoordvars: add $\mathrm{i}$ and $\mathrm{j}$ variables and assign them as coordinate variables.

- convert_units: unit conversions that cannot be handled by check_units.

- add_ECEARTHgrid_info: add ECEARTH grid info (lat, lon, areacello and grid sizes) to the output.

\section{Control flow of reformat_EMAC}

The workflow is similar to the default case, but some additional operations specific to the EMAC model are performed in addition:

- find messy version: the MESSy version with which the EMAC output has been generated is read from the data.

- find EMAC name: the EMAC name of the selected variable is found from the table in names_EMAC.dat (an error message is issued if not defined). For complex variables (i.e., variables not directly available as EMAC output but derivable from other EMAC variables), a user-defined recipe can be provided in reformat_scripts/EMAC/recipes/EMAC_recipes_xxx.ncl to derive it.

- check field consistency: reads from names_EMAC.dat file the list of allowed fields for the selected variable (for example is not possible to select total column ozone toz as T3M field).

- check vertical integration type (only for T2?s types): reads from names_EMAC.dat the option for the vertical coordinate ( $\mathrm{C}$ for column integration, $\mathrm{S}$ for surface value).

- $\quad$ start the time loop: the EMAC output is assumed to be monthly-aggregated (monthly averages are optional). The data are read starting from January of the start_year to December of the end_year.

- extract variable: the selected variable is searched in the EMAC output. If multiple files for a given month/year combination contain the selected variable, the following priority list is applied: time coordinate matching the field type (monthly mean or daily output), data from tracer_gp and $\mathrm{tr}_{-}{ }^{*}$ streams/channels, first file in the list. For complex variables, the corresponding user-defined recipe is called (reformat_scripts/EMAC/recipes/EMAC_recipes_xxx.ncl). For $\mathrm{T} 2$ ?z types, the data are interpolated on constant pressure levels (defined in reformat_scripts/constants.ncl).

- create time series: within the time loop, a time series start_year-end_year is created.

- reformat coordinates, check units, set variable attributes and write output: these operations are applied exactly as in the default case.

The user can extend the reformat_scripts/EMAC/recipes/EMAC_recipes_xxx.ncl in order to calculate additional (derived) variables not directly available in EMAC.

\section{Annex B - subversion, Mantis, wiki 12.1Subversion repository}

The ESMValTool development currently uses a subversion repository for managing and maintaining the source code.

\section{The ESMValTool repository is located at: https://svn.dlr.de/ESM-Diagnostic/} (use https://svn.dlr.de/viewvc/ESM-Diagnostic/source/ for an improved web interface) 
The following description gives an overview of the typical workflow and usage for implementing a new diagnostic into the ESMValTool. For general information on subversion see the online svn book at http://svnbook.red-bean.com/.

\subsubsection{General do's and don'ts}

Always use the "svn $r m<\ldots>$ ", "svn $m v<\ldots>$ " and "svn $c p<\ldots>$ " instead of $r m, m v$, and $c p$. That way subversion will be able to keep track changes applied to the (local copy of the) development branch.

Note that it is possible to apply the above commands directly to the repository, e.g., "svn copy $<U R L 1>$ $<U R L 2>$ ". This is useful when modifying folders higher up in the repository structure.

When merging, svn keeps track of the revisions merged via the svn:merginfo-metadata, i.e., it is (usually) not required to specify a revision range for a merge.

\subsubsection{Typical workflow}

NB: All of the following svn commands take the optional argument "--username <USERNAME $>$ " to explicitly specify your (DLR) username.

(1) Create a new branch by copying the latest version of the trunk into to your new branch on the repository, selecting the appropriate project (<your-project $>$ ) and choosing a new branch name $(<$ your-branch $>$ ):

svn copy -m "creating branch ..." https://svn.dlr.de/ESM-Diagnostic/source/trunk/ https://svn.dlr.de/ESM-Diagnostic/source/branches/<your-project $>/<$ your-branch $>$

(2) Checkout a local copy of your newly created branch (if you already have a <local-path>, you can just call svn update):

svn checkout https://svn.dlr.de/ESM-Diagnostic/source/branches/<your-project $>/<$ your-branch $>$ $<$ local-path>

(3) Work in the local folder and commit your changes on a regular basis to the repository:

svn commit -m "changed ...”

(4) Keep your development branch updated with the trunk, by bringing in trunk updates on a regular basis (recommended is at least once per week):

svn merge https://svn.dlr.de/ESM-Diagnostic/source/trunk --dry-run

The "--dry-run" option will give you a heads up to which files are about to be merged/updated and possible merge conflicts. NB: the --dry-run option does not display updates to the svn mergeinfo metadata. Updating the mergeinfo is always necessary and you should therefore, always, proceed with 5-c and 5-e even if the output from 5-a indicated no changes.

svn merge https://svn.dlr.de/ESM-Diagnostic/source/trunk

(5) Resolve any merge conflicts that were reported in step 4, then test the local changes (important) and commit your changes back to your branch with:

svn commit -m "implemented the following changes: ..." 
NB: Always follow through with the svn commit even if no files were updated. The svn:merginfo has been updated and needs to be commited back to your branch.

(6) Reintegrate your work to trunk by (core developers only, other users shall do a merge request to the core development team, see Responsibilities for ESMValTool Development Team):

- switch your working folder to trunk: svn switch https://svn.dlr.de/ESM-Diagnostic/source/trunk

- $\quad$ cd to the trunk (--dry-run means try operation but make no changes),

- svn merge --reintegrate https://svn.dlr.de/ESM-Diagnostic/source/<branch-URL >/featurebranch-name --dry-run

- do the actual reintegration: svn merge --reintegrate https://svn.dlr.de/ESMDiagnostic/source/<branch-URL>/feature-branch-name

(7) Test the changes locally (very important to keep the trunk stable), then commit. If a Mantis issue exists (see also section 12.2) it can be useful to refer to this in the commit comment.

svn commit -m "merging feature-branch-name (Mantis \#XXXXX) to the trunk"

(8) At this point the reintegrated branch contains erroneous meta data and should not be used further. In order to prepare removal of the local copy, switch back to the feature branch:

svn switch https://svn.dlr.de/ESM-Diagnostic/source/<branch-URL>/feature-branch-name

Then either run

svn $r m<$ branch-URL $>$ /feature-branch-name

or, if it is worth to keep an easy access point to the branch in the repository, move it to the legacy folder:

svn mv -m "moving feature-branch-name into legacy" <branch-URL>/feature-branch-name $<$ branch-legacy-folder $>$

Finally remove your local copy with

svn update

and - if needed - manually delete files that were not under version control.

\subsubsection{Other common svn commands}

- svn status: check status of changes to local files, e.g., files modified, moved, gone missing, etc.

- $\quad$ svn status -uv: verbose output (-v) + indicate updates in the repository with a ${ }^{\prime * 1}(-\mathrm{u})$

- svn info: general info about the checked out files + details which repository URL the local files represent

- svn update: bring in current URL updates from the repository

- svn diff: check the difference between local edits and their original (= locally unmodified) state. Note: this diff is never done towards the repository files, see 'svn revert' below. 
- svn ls $<U R L>$ : list content of the repository $<\mathrm{URL}>$

- svn revert <file>: undo local changes, i.e., the ones shown by 'svn status' and 'svn diff. The file is reverted to its original state. Note: the original state is the locally unmodified state, i.e., the state the file had at the time of the previous update / commit. If another developer has committed an update of the same file to the repository then the original state will differ from the repository state.

- svn rm: svn "aware" 'rm'-command

- $\quad$ svn mv: svn "aware" 'mv'-command

- svn cp: svn "aware" 'cp'-command

\subsection{Mantis bug tracker}

The ESMValTool developers currently use the Mantis bug tracker (http://www.mantisbt.org/) to discuss any kind of problems and open issues encountered with the ESMValTool. The ESMValTool Mantis page can be found at: https://mantis.dlr.de/mantis/my view page.php

\subsection{Wiki}

The latest information on the ESMValTool and diagnostics under development can be found on the wiki available at https://teamsites-extranet.dlr.de/pa/ESMValTool/Wiki/Home.aspx. All users and developers are strongly encouraged to frequently check the ESMValTool wiki for new information, contact data or observational data.

\section{Annex C - Available diagnostics and metrics}

The following pages give an overview on the available diagnostics and metrics packages implemented into the ESMValTool v1.0. The overview contains a brief description of the diagnostic/metric, a list of available user settings, information on the observational data used, references, and example plots.

For further information on the diagnostics and performance metrics of ESMValTool v1.0, see also

Eyring et al., ESMValTool (v1.0) - a community diagnostic and performance metrics tool for routine evaluation of Earth System Models in CMIP, Geosci. Model Dev., 2016. 


\section{Aerosol}

\section{Overview}

The aerosol diagnostics currently implemented allow for three kinds of comparisons:

- station data: concentrations of various aerosol species (aerosol sulfate, aerosol nitrate, aerosol ammonium, black carbon, organic carbon, $\mathrm{PM}_{2.5}$ and $\mathrm{PM}_{10}$ ) are compared with observational data from station networks (IMPROVE and CASTNET for North America, EMEP for Europe and EANET for Asia). Aerosol optical depth (AOD) at $550 \mathrm{~nm}$ is compared with AERONET data. The comparison between model and observations is performed considering all available observational data in the selected time period, on a monthly-mean basis. The model data is extracted in the grid boxes where the respective observational stations are located (co-located model and observational data). This diagnostic produces three types of plot: time series (model/observations vs. time), scatter plot (model versus observations) and a map plot (model as contour map, observations as dots using the same color coding).

- satellite data: AOD at $550 \mathrm{~nm}$ is compared with satellite data (MODIS and MISR). This diagnostic produces contour map plots of the AOD of model and observations, as well as difference plot between model and observations.

- vertical profiles, size distributions: vertical profiles of aerosol (mass and number) concentrations and of aerosol size distributions are compared to observational data from aircraft campaigns and ground based stations. The model data are extracted based on the campaign/station location (lat-lon box) and time period (on a climatological basis, i.e. selecting the same days/months, but regardless of the year). The basic statistics is calculated on the selected model data (mean, standard deviation, median, 5-10-25-75-90-95 percentiles) and compared to the same quantities from the observations (where available). This diagnostics supports monthly-mean, daily-mean and instantaneous input data, with the latter two providing a more robust evaluation. For this diagnostic, rather specific variables are required (i.e., aerosol number concentration for particles with diameter larger than $14 \mathrm{~nm}$ ) to match the properties of the instruments used during the campaign. New CMOR variables have been added and corresponding EMAC recipes have been defined.

\section{Available Namelists and Diagnostics}

Namelists are stored in $\mathrm{nml} /$

- namelist_aerosol_CMIP5.xml

- namelist_aerosol_EMAC.xml

Diagnostics are stored in diag_scripts/

- aerosol_stations.ncl

- aerosol_satellite.ncl

- aerosol_profiles.ncl 


\section{User settings}

User setting files (cfg files) are stored in $\mathrm{nml} / \mathrm{cfg} \_$aerosol/CMIP5/ and nml/cfg_aerosol/EMAC/

! NOTE ! The cfg files may contain hard coded pathnames (attribute “datapath", see below) for the observational data that have to be set by the user!

(1) aerosol_stations.ncl (AERONET, CASTNET, EANET, EMEP)

- cfg_aerosol_stations_AERONET.ncl

- cfg_aerosol_stations_CASTNET.ncl

- cfg_aerosol_stations_EANET.ncl

- cfg_aerosol_stations_EMEP.ncl

- cfg_aerosol_tsline.ncl

Required diag_script_info attributes

o network: name of measurement network (AERONET, CASTNET, EANET, EMEP, IMPROVE)

o time_avg: time averaging (monthly, yearly)

o styleset: "DEFAULT"

o datapath: path for station data

o legend_outside: plot legend inside/outside of plot (True, False)

(2) aerosol_satellites.ncl (ESACCI, MISR, MODIS)

- satellite_ESACCI-AEROSOL.ncl

- cfg_aerosol_satellite_MISR.ncl

- cfg_aerosol_satellite_MODIS.ncl

Required diag_script_info attributes

o ref_model: name of reference data set (e.g., "ESACCI-AEROSOL")

o styleset: style ("DEFAULT")

o regrid_method: regridding method (coarsest, finest, ref)

o range_option: time range option ( 0 : model own time range, 1 : intersection between models)

(3) aerosol_profiles.ncl (CIRRUS, CONCERT, CR-AVE, DC3, HIPPO, IMPROVE, INCA, LACE, Melpitz, Putaud, SALTRACE, TC4, Texas, UCN-Pacific)

- cfg_aerosol_profiles_CIRRUS.ncl

- cfg_aerosol_profiles_CONCERT.ncl

- cfg_aerosol_profiles_CR-AVE.ncl

- cfg_aerosol_profiles_DC3.ncl

- cfg_aerosol_profiles_HIPPO.ncl 
- cfg_aerosol_stations_IMPROVE.ncl

- cfg_aerosol_profiles_INCA.ncl

- cfg_aerosol_profiles_LACE.ncl

- cfg_aerosol_profiles_Melpitz.ncl

- cfg_aerosol_profiles_Putaud.ncl

- cfg_aerosol_profiles_SALTRACE.ncl

- cfg_aerosol_profiles_TC4.ncl

- cfg_aerosol_profiles_Texas.ncl

- cfg_aerosol_profiles_UCN-Pacific.ncl

Required diag_script_info attributes

o campaign: name of measurement campaign (e.g., INCA, LACE, Texas)

o styleset: "DEFAULT"

o datapath: path for campaign data

o cc_threshold: cloud cover threshold (\%)

\section{Variables}

\section{CMIP5}

- $\quad$ sconcbc, sconcoa, sconcso4

- od550aer

EMAC

- conccnmode, diamcnmode, cl

- conccnSTPd3, concenSTPd5, concenSTPd14, concenSTPd120

- mmrbc, mmraer, mmrbcfree

- sconcso4, sconcno3, sconcnh4, sconcbc, sconcoa, sconcpm10, sconcpm2p5

- od550aer

\section{Observations and Reformat Scripts}

Note: (1) obs4mips data can be used directly without any preprocessing; (2) see headers of reformat scripts for non-obs4mips data for download instructions.

- AERONET (reformat_scripts/obs/reformat_obs_AERONET.ncl)

- CASTNET (reformat_scripts/obs/reformat_obs_CASTNET.ncl)

- EANET (reformat_scripts/obs/reformat_obs_EANET.ncl)

- EMEP (reformat_scripts/obs/reformat_obs_EMEP.ncl) 
- IMPROVE (reformat_scripts/obs/reformat_obs_IMPROVE.ncl)

- MODIS (obs4mips)

- $\quad$ MISR (obs4mips)

- ESACCI-AEROSOL (reformat_scripts/obs/reformat_obs_ESACCI-AEROSOL.ncl)

- ACCESS (reformat_scripts/obs/reformat_obs_ACCESS.ncl)

- CIRRUS (reformat_scripts/obs/reformat_obs_CIRRUS.ncl)

- CONCERT (reformat_scripts/obs/reformat_obs_CONCERT.ncl)

- CR-AVE (reformat_scripts/obs/reformat_obs_CR-AVE.ncl)

- DC3 (reformat_scripts/obs/reformat_obs_DC3.ncl)

- HIPPO (reformat_scripts/obs/reformat_obs_HIPPO.ncl)

- INCA (reformat_scripts/obs/reformat_obs_INCA.ncl)

- LACE (reformat_scripts/obs/reformat_obs_LACE.ncl)

- Putaud (reformat_scripts/obs/reformat_obs_Putaud.ncl)

- SALTRACE (reformat_scripts/obs/reformat_obs_SALTRACE.ncl)

- TC4 (reformat_scripts/obs/reformat_obs_TC4.ncl)

- Texas (reformat_scripts/obs/reformat_obs_Texas.ncl)

- UCN-Pacific (reformat_scripts/obs/reformat_obs_UCN-Pacific.ncl)

\section{References}

Aquila, V. et al., MADE-in: a new aerosol microphysics submodel for global simulation of insoluble particles and their mixing state. Geosci. Model Dev. 4 (2), 325-355 (2011).

Lauer, A. et al., Simulating aerosol microphysics with the EMAC/MADE GCM - Part I: Model description and comparisons with observations. Atmos. Chem. Phys. 5 (12), 3251-3276 (2005).

Righi, M. et al.. The global impact of the transport sectors on atmospheric aerosol: Simulations for year 2000 emissions. Atmos. Chem. Phys. 13 (19), 9939-9970 (2013). 


\section{Example plots}
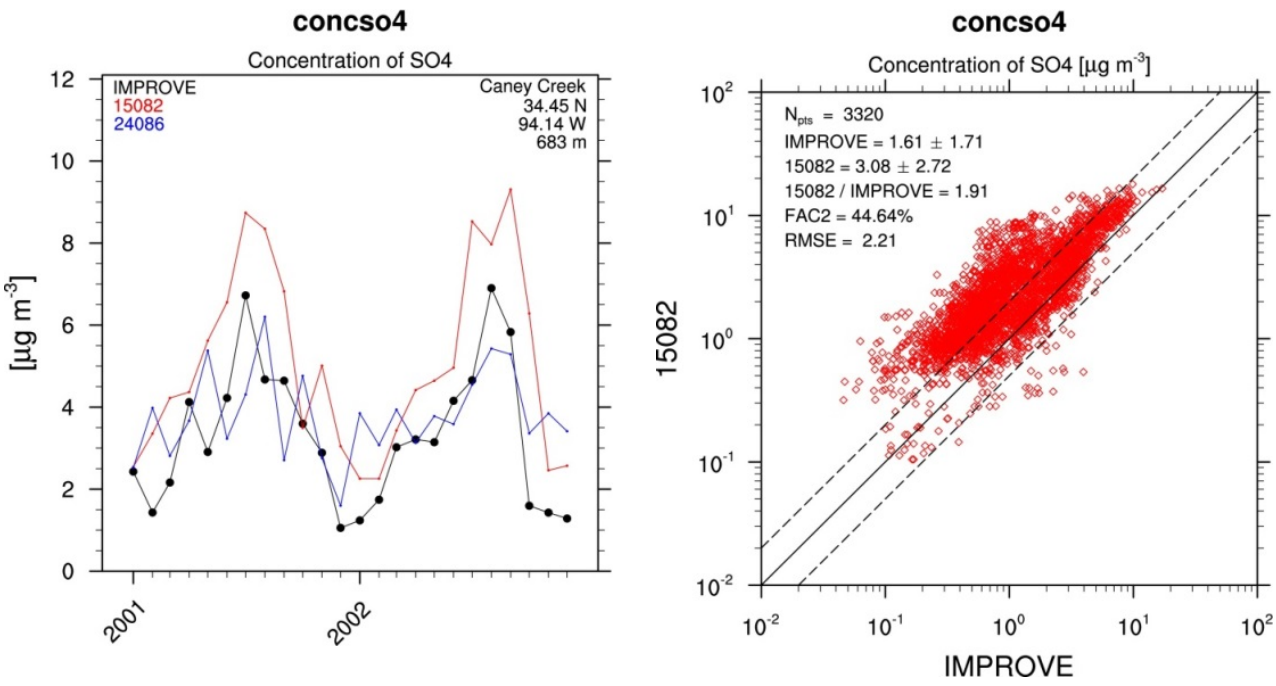

concso4 (24086 - IMPROVE)

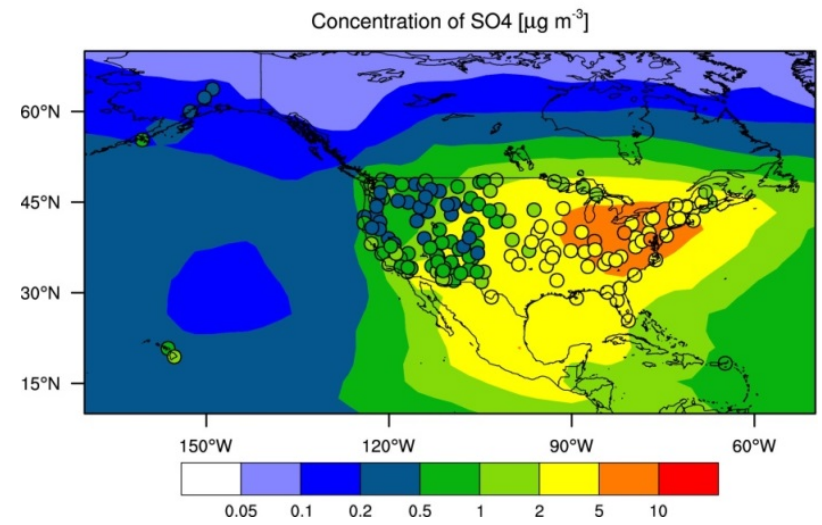

od550aer (MODIS)

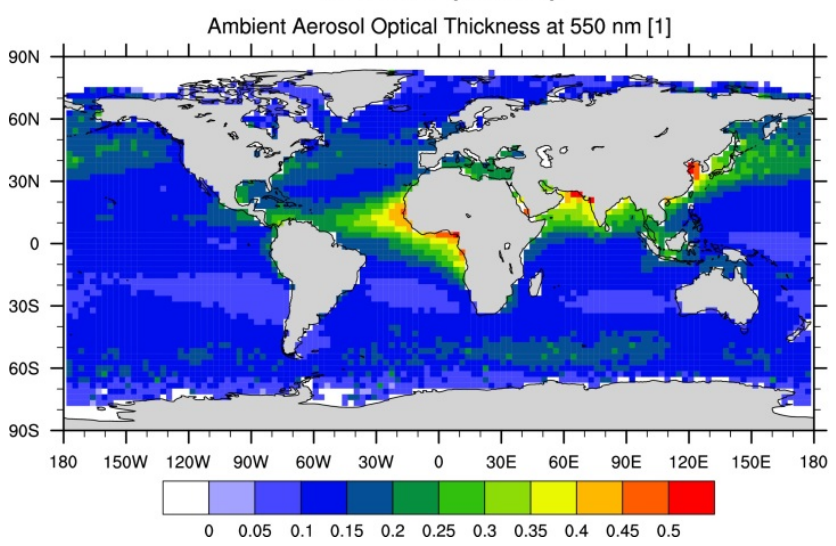

od550aer (24086 - MODIS)

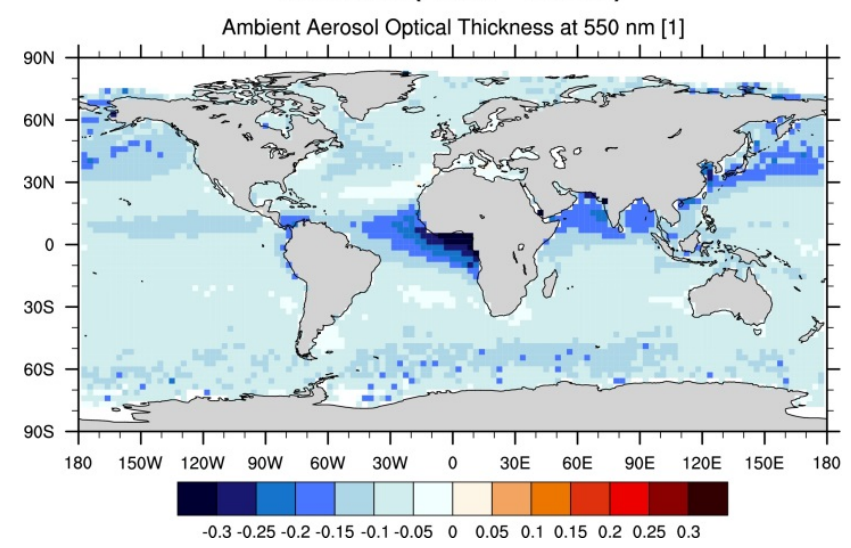



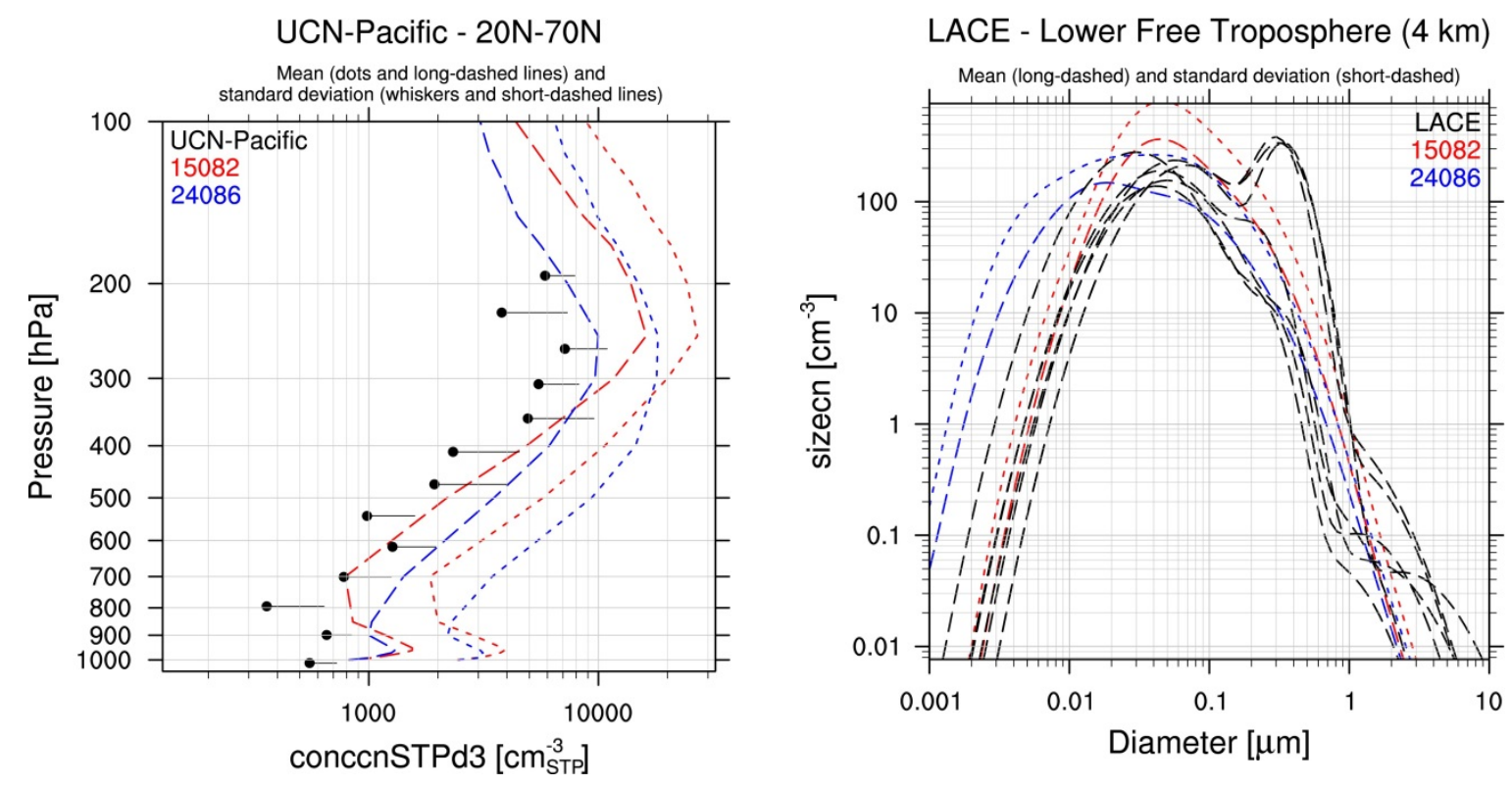


\section{Catchment analysis}

\section{Overview}

This diagnostic calculates biases of long-term climatological annual means of total runoff, precipitation and evapotranspiration for 12 large-scale catchments on different continents and climates. For total runoff, catchment averaged model values are compared to climatological GRDC station observations of river runoff (Dümenil Gates et al., 2000). Due to the incompleteness of these station data, a year-to-year correspondence of data cannot be achieved in a generalized way, so that only climatological data are considered, such it has been done in Hagemann et al. (2013). For precipitation, catchment-averaged WFDEI precipitation (Weedon et al., 2014) data from 1979-2010 is used as reference. For evapotranspiration, observations are estimated using the difference of the above mentioned precipitation reference minus the climatological GRDC river runoff.

\section{Available Namelists and Diagnostics}

Namelists are stored in $\mathrm{nml} /$

- namelist_runoff_et.xml

Diagnostics are stored in diag_scripts/

- catchment_analysis_val.py

\section{User settings}

None.

\section{Variables}

- mrro (land, annual mean, longitude latitude time)

- evspsbl (atmos, annual mean, longitude latitude time)

- $\operatorname{pr}($ atmos, annual mean, longitude latitude time)

\section{Observations and Reformat Scripts}

The observational data (big_catchments.nc) are provided with the ESMValTool and are located in diag_scripts/aux/catchment_analysis/.

- Climatological river runoff from various GRDC stations

- WFDEI precipitation (Weedon et al., 2014). 


\section{References}

Dümenil Gates, L., S. Hagemann and C. Golz, 2000: Observed historical discharge data from major rivers for climate model validation. Max Planck Institute for Meteorology Report 307, Hamburg, Germany.

Hagemann, S., A. Loew, A. Andersson, 2013: Combined evaluation of MPI-ESM land surface water and energy fluxes. J. Adv. Model. Earth Syst., 5: 259-286, doi: 10.1029/2012MS000173.

Weedon, G. P., G. Balsamo, N. Bellouin, S. Gomes, M. J. Best, and P. Viterbo (2014), The WFDEI meteorological forcing data set: WATCH Forcing Data methodology applied to ERA-Interim reanalysis data, Water Resour. Res., 50, 7505-7514, doi: 10.1002/2014WR015638.

\section{Example Plots}
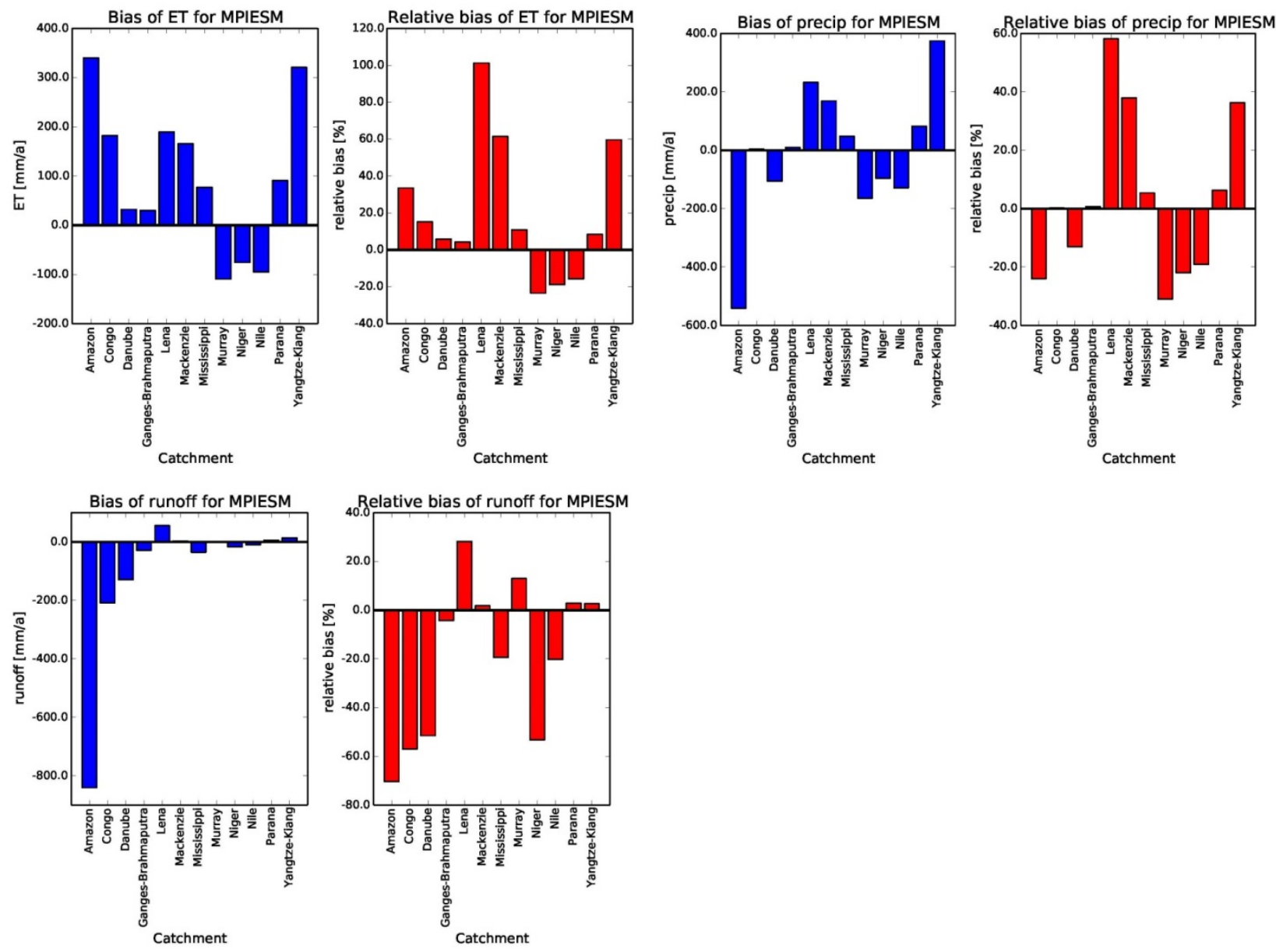

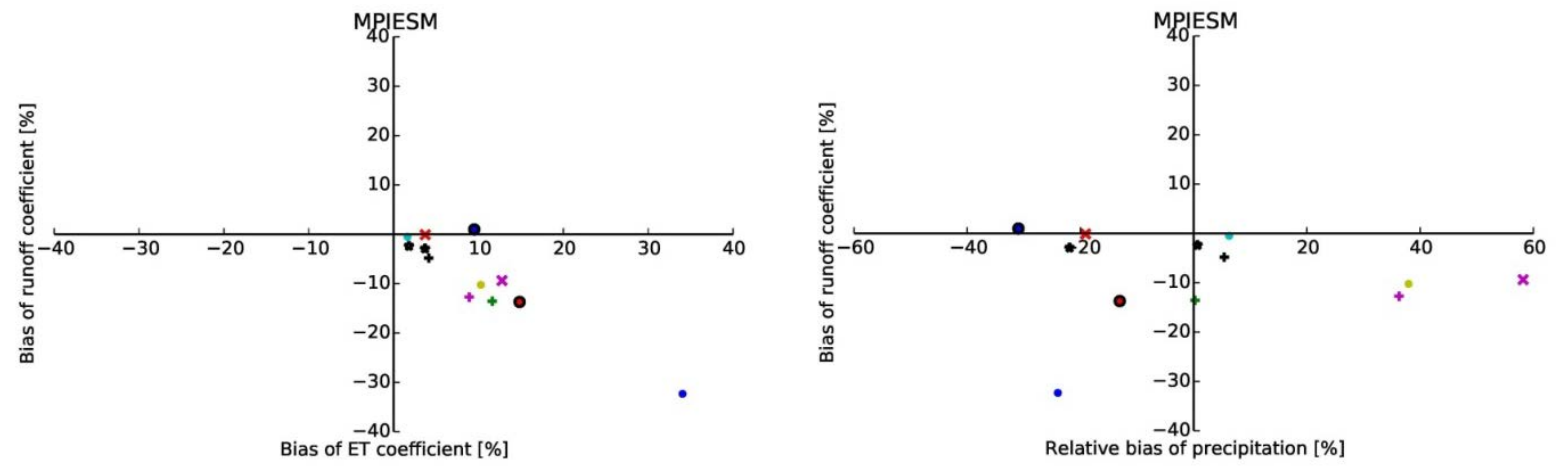

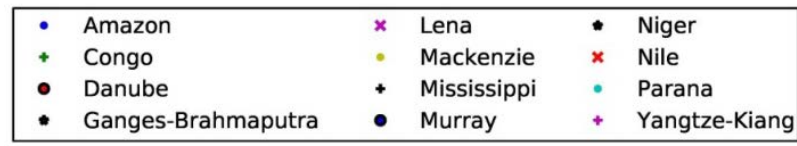

\begin{tabular}{|c|c|c|c|c|c|}
\hline - & Amazon & $x$ & Lena & & Niger \\
\hline+ & Congo & - & Mackenzie & $x$ & Nile \\
\hline - & Danube & + & Mississippi & • & Parana \\
\hline - & Ganges-Brahmaputra & • & Murray & + & Yangtze-Kiang \\
\hline
\end{tabular}




\section{Clouds}

\section{Overview}

The namelist namelist_lauer13jclim.xml computes the climatology and interannual variability of climate relevant cloud variables such as cloud radiative forcing (CRE), liquid (lwp) and ice water path (iwp), cloud amount (clt), and total precipitation (pr) reproducing some of the evaluation results of Lauer and Hamilton (2013). The standard namelist includes a comparison of the geographical distribution of multi-year average cloud parameters from individual models and the multi-model mean with satellite observations. Taylor diagrams are generated that show the multi-year annual or seasonal average performance of individual models and the multi-model mean in reproducing satellite observations. The diagnostic also facilitates the assessment of the bias of the multi-model mean and zonal averages of individual models compared with satellite observations. Interannual variability is estimated as the relative temporal standard deviation from multi-year timeseries of data with the temporal standard deviations calculated from monthly anomalies after subtracting the climatological mean seasonal cycle.

\section{Available Namelists and Diagnostics}

Namelists are stored in $\mathrm{nml} /$

- namelist_lauer13jclim.xml

Diagnostics are stored in diag_scripts/

- clouds.ncl: global maps of (multi-year) annual means including multi-model mean

- clouds_bias.ncl: global maps of the multi-model mean and the multi-model mean bias

- clouds_interannual: global maps of the interannual variability

- clouds_isccp: global maps of multi-model mean minus observations + zonal averages of individual models, multi-model mean and observations

- clouds_taylor.ncl: taylor diagrams

\section{User settings}

User setting files (cfg files) are stored in $\mathrm{nml} / \mathrm{cfg}$ _cloud/

(1) clouds.ncl: cfg_clouds.ncl

Required diag_script_info attributes

o grid: grid for regridding (coarsest, finest, ref) in case calculation of the multi-model mean is requested

o ref_model: name of reference data set

Optional diag_scipt_info attributes

o calcmm: calculate multi-model mean (True, False) 
o colormap: e.g., WhiteBlueGreenYellowRed, rainbow

o ncdf: enable to output to netCDF; either use "default" or give a full file name

o projection: map projection, e.g., Mollweide, Mercator (see http://www.ncl.ucar.edu/Document/Graphics/Resources/mp.shtml\#mpProjection for available projections)

o styleset: "CMIP5", "DEFAULT"

o timemean: time averaging, i.e. "seasonal” (DJF, MAM, JJA, SON), "annual” (annual mean) Color tables

o variable "lwp": diag_scripts/lib/ncl/rgb/qcm3.rgb

(2) clouds.ncl: cfg_clouds_bias.ncl

Required diag_script_info attributes

o grid: grid for regridding (coarsest, finest, ref) in case calculation of the multi-model mean is requested

o ref_model: name of reference data set

o valid_fraction: minimum fraction of valid data points (for creating a mask)

Optional diag_scipt_info attributes

o colormap (for variables other than tas and pr-mmday): e.g., WhiteBlueGreenYellowRed, rainbow

o ncdf: enable to output to netCDF; either use "default" or give a full file name

o projection: map projection, e.g., Mollweide, Mercator (see http://www.ncl.ucar.edu/Document/Graphics/Resources/mp.shtml\#mpProjection for available projections)

o styleset: "CMIP5", "DEFAULT"

Color tables

o variable "tas": diag_scripts/lib/ncl/rgb/ipcc-tas.rgb, diag_scripts/lib/ncl/rgb/ipcc-tasdelta.rgb

o variable "pr-mmday": diag_scripts/lib/ncl/rgb/ipcc-precip-delta.rgb

(3) clouds_interannual.ncl: cfg_clouds_interannual.ncl

Required diag_script_info attributes

o grid: grid for regridding (coarsest, finest, ref) in case calculation of the multi-model mean is requested

o ref_model: name of reference data set

Optional diag_scipt_info attributes

o calcmm: calculate multi-model mean (True, False)

o colormap (for variables other than lwp): e.g., WhiteBlueGreenYellowRed, rainbow 
o ncdf: enable to output to netCDF; either use "default" or give a full file name

o projection: map projection, e.g., Mollweide, Mercator (see http://www.ncl.ucar.edu/Document/Graphics/Resources/mp.shtml\#mpProjection for available projections)

o styleset: "CMIP5", "DEFAULT"

Color tables

o variable "lwp": diag_scripts/lib/ncl/rgb/qcm3.rgb

(4) clouds_ipcc.ncl: cfg_clouds_ipcc.ncl

Required diag_script_info attributes

o grid: grid for regridding (coarsest, finest, ref) in case calculation of the multi-model mean is requested

o ref_model: name of reference data set

o valid_fraction: minimum fraction of valid data points (for creating a mask)

Optional diag_scipt_info attributes

o calcmm: calculate multi-model mean (True, False)

o colormap (for variables other than pr and pr-mmday): e.g., WhiteBlueGreenYellowRed, rainbow

o ncdf: enable to output to netCDF; either use "default" or give a full file name

o projection: map projection, e.g., Robinson, CylindricalEquidistant, Mollweide, Mercator (see http://www.ncl.ucar.edu/Document/Graphics/Resources/mp.shtml\#mpProjection for available projections)

o styleset: "CMIP5", "DEFAULT"

o timemean: time averaging, i.e. "seasonal” (DJF, MAM, JJA, SON), "annual" (annual mean)

Color tables

O variables "pr", “pr-mmday": diag_scripts/lib/ncl/rgb/ ipcc-precip-delta.rgb

(5) clouds_taylor.ncl: cfg_clouds_taylor.ncl

Required diag_script_info attributes

o grid: grid for regridding (coarsest, finest, ref) in case calculation of the multi-model mean is requested

o ref_model: name of reference data set

o valid_fraction: minimum fraction of valid data points (for creating a mask)

Optional diag_scipt_info attributes

o calcmm: calculate multi-model mean (True, False)

o ncdf: enable to output to netCDF; either use "default" or give a full file name

o styleset: "CMIP5", "DEFAULT" 
o timemean: time averaging, i.e. "seasonal” (DJF, MAM, JJA, SON), "annual" (annual mean)

\section{Variables}

- clwvi (atmos, monthly mean, longitude latitude time)

- clivi (atmos, monthly mean, longitude latitude time)

- $\quad$ clt (atmos, monthly mean, longitude latitude time)

- $\operatorname{pr}$ (atmos, monthly mean, longitude latitude time)

- rlut, rlutcs (atmos, monthly mean, longitude latitude time)

- rsut, rsutcs (atmos, monthly mean, longitude latitude time)

\section{Observations and Reformat Scripts}

Note: (1) obs4mips data can be used directly without any preprocessing; (2) see headers of reformat scripts for non-obs4mips data for download instructions.

- CERES-EBAF (obs4mips) - CERES TOA radiation fluxes (used for calculation of cloud forcing)

- GPCP-SG (obs4mips) - Global Precipitation Climatology Project total precipitation

- MODIS (obs4mips) - MODIS total cloud fraction

- MODIS-CFMIP - MODIS ice water path climatology, originally created for CFMIP, references: King et al. (2003), IEEE Trans. Geosci. Remote Sens.; Pincus et al. (2012), J. Clim.

Reformat script: reformat_scripts/obs/reformat_obs_MODIS-CFMIP.ncl

- UWisc - University of Wisconsin-Madison liquid water path climatology, based on satellite observbations from TMI, SSM/I, and AMSR-E, reference: O'Dell et al. (2008), J. Clim.

Reformat script: reformat_scripts/obs/reformat_obs_UWisc.ncl

\section{References}

King, M. D., et al. (2003), Cloud and aerosol properties, precipitable water, and profiles of temperature and water vapor from MODIS, IEEE Trans. Geosci. Remote Sens., 41, 442-458, doi: 10.1109/TGRS.2002.808226.

Lauer A., and K. Hamilton (2013), Simulating clouds with global climate models: A comparison of CMIP5 results with CMIP3 and satellite data, J. Clim., 26, 3823-3845, doi: 10.1175/JCLI-D-12-00451.1.

O’Dell, C. W., F. J. Wentz, and R. Bennartz (2008), Cloud liquid water path from satellite-based passive microwave observations: A new climatology over the global oceans, J. Clim., 21, 1721-1739, doi:10.1175/2007JCLI1958.1.

Pincus, R., S. Platnick, S. A. Ackerman, R. S. Hemler, Robert J. Patrick Hofmann (2012), Reconciling simulated and observed views of clouds: MODIS, ISCCP, and the limits of instrument simulators. J. Climate, 25, 4699-4720, doi: 10.1175/JCLI-D-11-00267.1. 


\section{Example Plots}
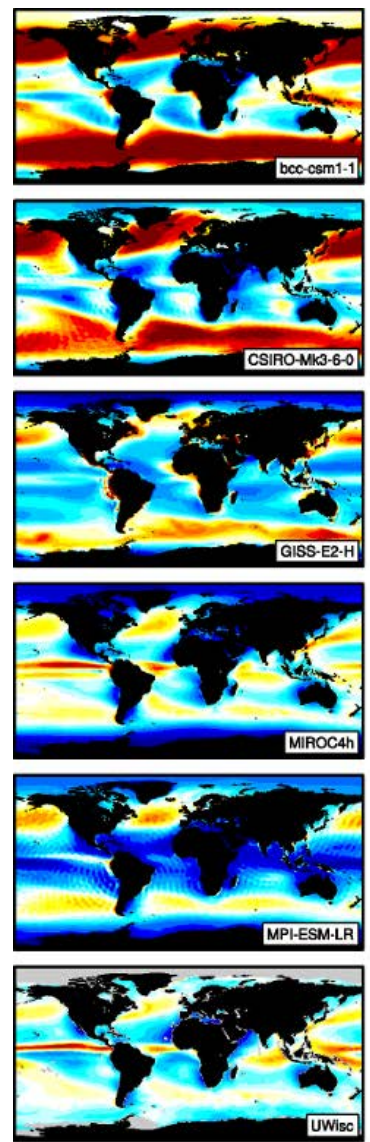
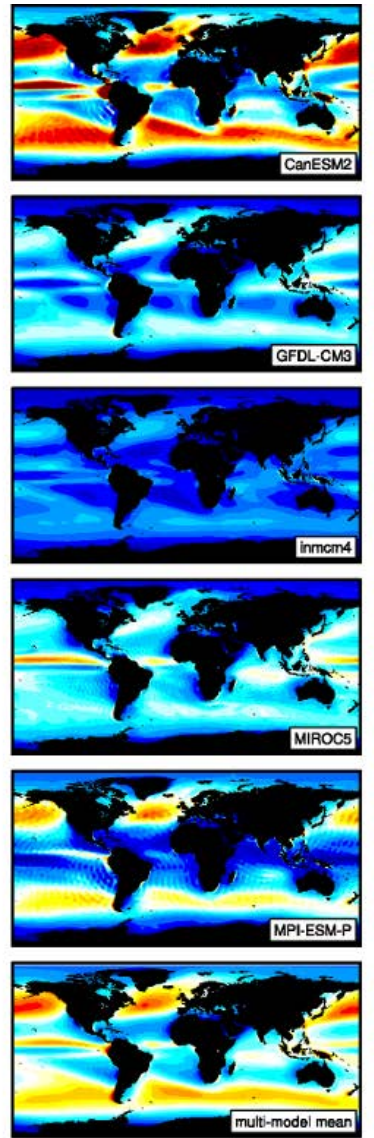
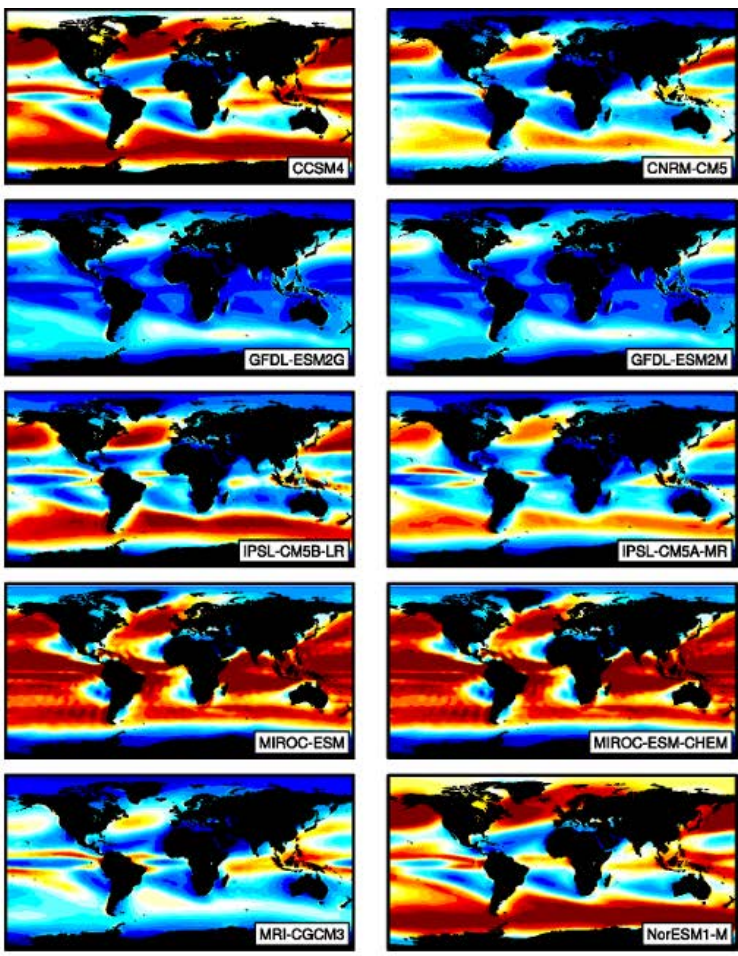

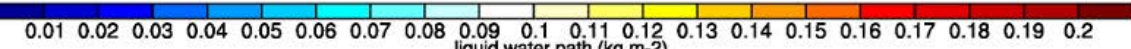

Figure 4 The 20-yr average LWP (1986-2005) from the CMIP5 historical model runs and the multi-model mean in comparison with the UWisc satellite climatology (1988-2007) based on SSM/I, TMI, and AMSR-E (O'Dell et al. 2008).

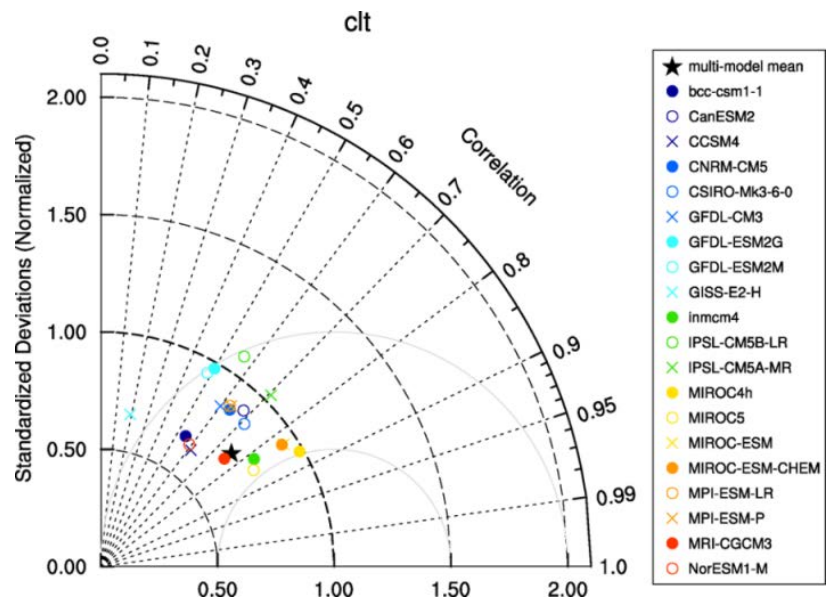

Figure 5 Taylor diagram showing the 20-yr annual average performance of CMIP5 models for total cloud fraction as compared to MODIS satellite observations. 

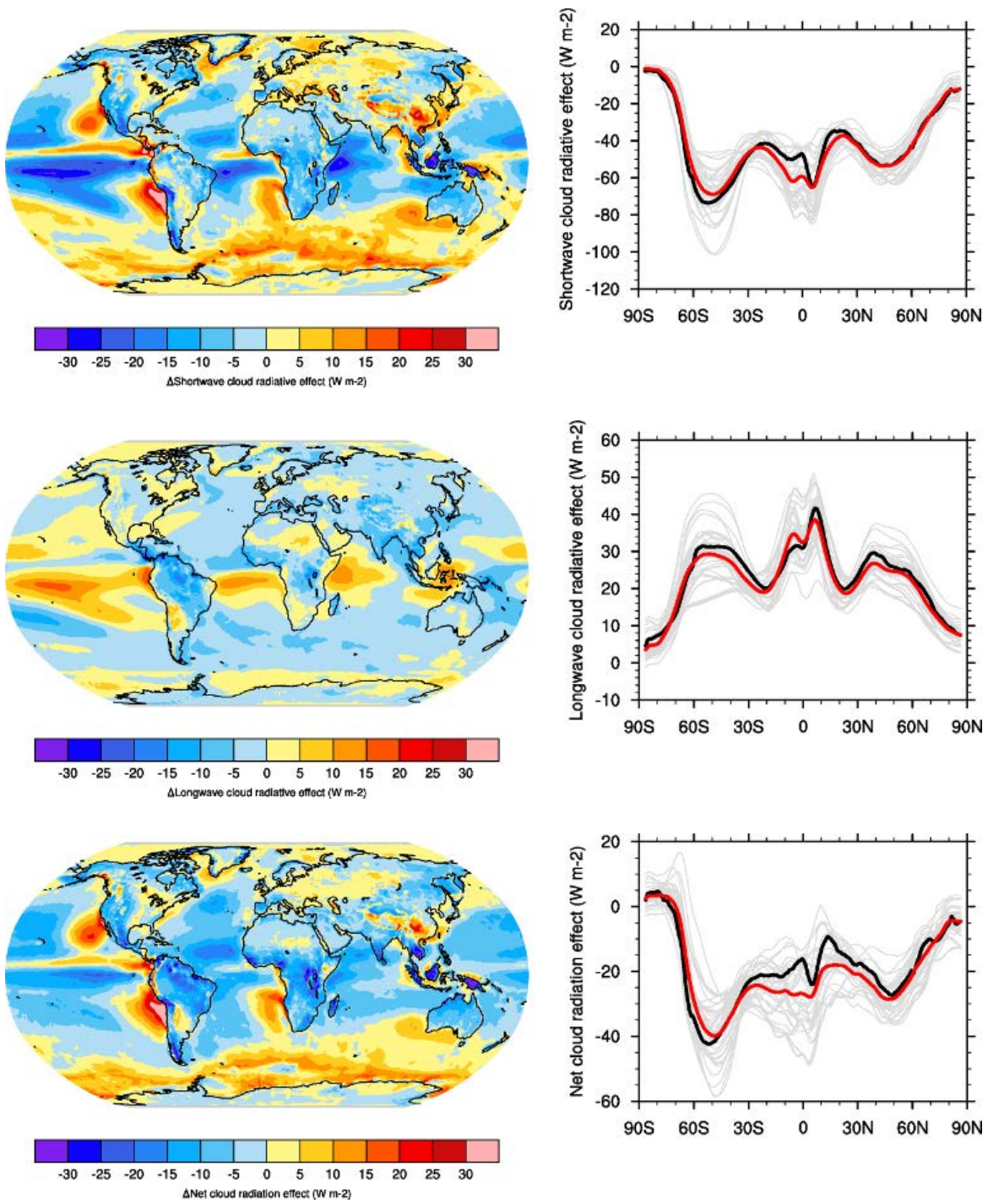

Figure 6 20-year average (1986-2005) annual mean cloud radiative effects of CMIP5 models against the CERES EBAF (2001-2012). Top row shows the shortwave effect; middle row the longwave effect, and bottom row the net effect. Multi-model mean biases against CERES EBAF are shown on the left, whereas the right panels show zonal averages from CERES EBAF (thick black), the individual CMIP5 models (thin gray lines) and the multi-model mean (thick red line). Similar to Figure 9.5 of Flato et al. (2013). 

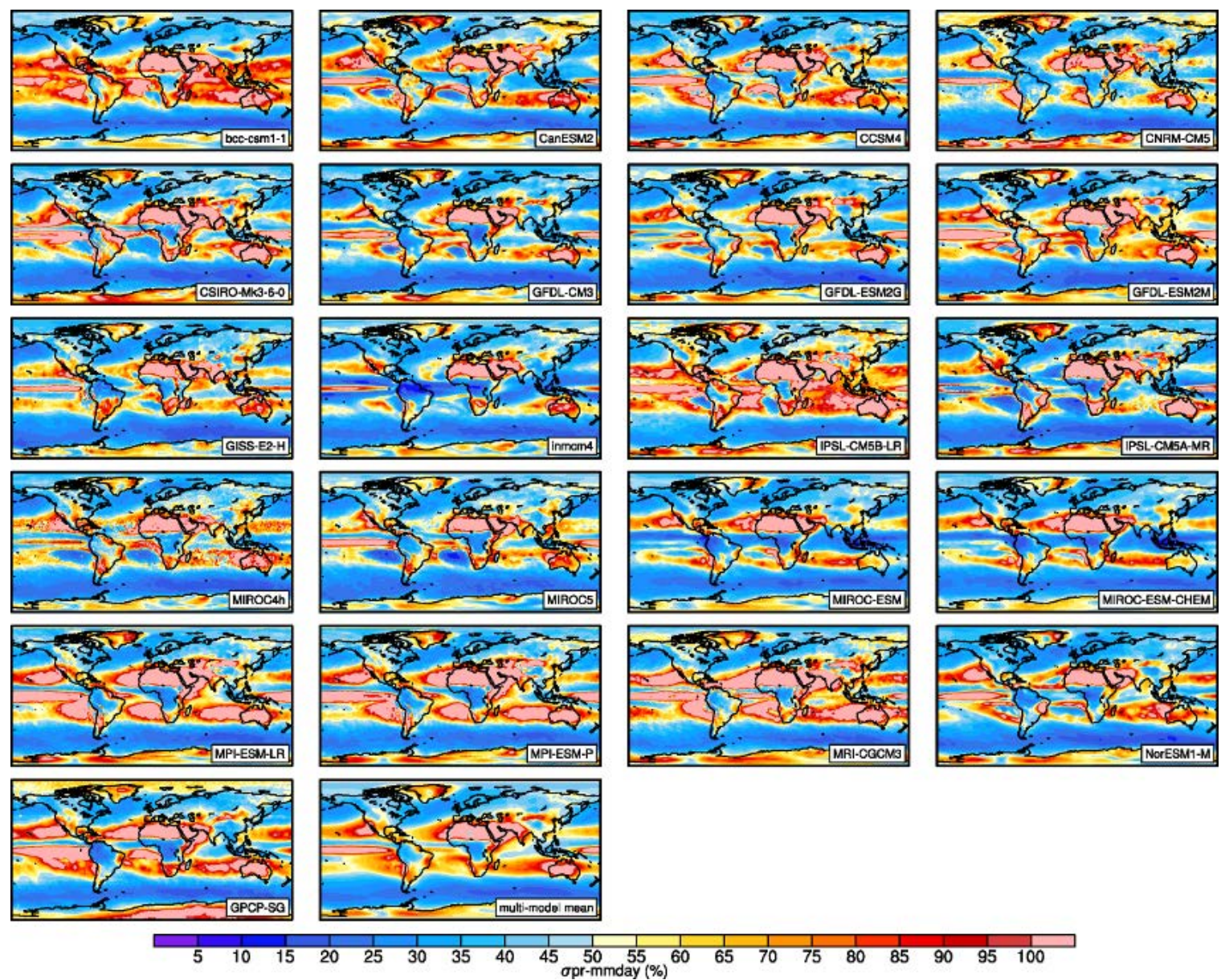

Figure 7 Interannual variability of modeled and observed (GPCP) precipitation rates estimated as relative temporal standard deviation from 20 years (1986-2005) of data. The temporal standard devitions are calculated from monthly anomalies after subtracting the climatological mean seasonal cycle. 


\section{Cloud Regime Error Metric (CREMpd)}

\section{Overview}

The radiative feedback from clouds remains the largest source of uncertainty in determining the climate sensitivity. Traditionally, cloud has been evaluated in terms of its impact on the mean top of atmosphere fluxes. However it is quite possible to achieve good performance on these criteria through compensating errors, with boundary layer clouds being too reflective but having insufficient horizontal coverage being a common example (e.g., Nam et al., 2012). Williams and Webb (2009) (WW09) propose a Cloud Regime Error Metric (CREM) which critically tests the ability of a model to simulate both the relative frequency of occurrence and the radiative properties correctly for a set of cloud regimes determined by the daily mean cloud top pressure, cloud albedo and fractional coverage at each grid-box. WW09 describe in detail how to calculate their metrics and we have included the CREMpd metric from their paper in ESMValTool, with clear references in the lodged code to tables in their paper. This has been applied to those CMIP5 models who have submitted the required diagnostics for their AMIP simulation (see Figure 8 below). As documented by WW09, a perfect score with respect to ISCCP would be zero. WW09 also compared MODIS/ERBE to ISCCP in order to provide an estimate of observational uncertainty. This was found to be 0.96 and this is marked on Figure 8, hence a model with a CREM similar to this value could be considered to have an error comparable with observational uncertainty, although it should be noted that this does not necessarily mean that the model lies within the observations for each regime. A limitation of the metric is that it requires a model to be good enough to simulate each regime. If a model is that poor that the simulated frequency of occurrence of a particular regime is zero, then a $\mathrm{NaN}$ will be returned from the code and a bar not plotted on the figure for that model.

\section{Available Namelists and Diagnostics}

Namelists are stored in $\mathrm{nml} /$

- namelist_williams09climdyn_CREM.xml

Diagnostics are stored in diag_scripts/

- ww09_ESMValTool.py

\section{User settings}

None.

\section{Variables}

- albisccp (atmos, daily mean, longitude latitude time)

- $\operatorname{cltisccp}$ (atmos, daily mean, longitude latitude time)

- $\quad$ pctisccp (atmos, daily mean, longitude latitude time)

- rlut (atmos, daily mean, longitude latitude time) 
- rlutcs (atmos, daily mean, longitude latitude time)

- $\quad$ rsut (atmos, daily mean, longitude latitude time)

- $\quad$ rsutcs (atmos, daily mean, longitude latitude time)

- $\quad$ sic (atmos, daily mean, longitude latitude time)

- $\quad$ snc (atmos, daily mean, longitude latitude time)

If snc is not available then snw can be used instead. For AMIP simulations, sic is often not submitted as it's a boundary condition and effectively the same for every model. In this case the same daily sic data set can be used for each model.

Note: in case of using sic data from a different model (AMIP), it has to be checked by the user that the calendar definitions of all data sets are compatible, in particular whether leap days are included or not.

\section{Observations and Reformat Scripts}

All observational data have been pre-processed and included within the routine. These are ISCCP, ISCCPFD, MODIS, ERBE. No additional observational data are required at runtime.

\section{References}

Williams, K.D. and Webb, M.J.: A quantitative performance assessment of cloud regimes in climate models. Clim. Dyn. 33, 141-157, doi: 10.1007/s00382-008-0443-1, 2009.

\section{Example Plots}

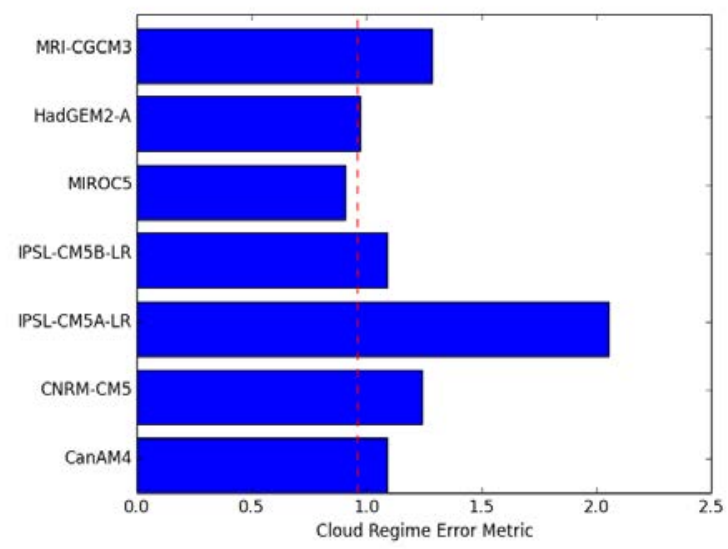

Figure 8 Cloud Regime Error Metrics (CREMpd) from William and Webb (2009) applied to those CMIP5 AMIP simulations with the required data in the archive. A perfect score with respect to ISCCP is zero; the dashed red line is an indication of observational uncertainty. 


\section{Diurnal cycle of convection}

\section{Overview}

The diurnal cycle of precipitation and radiative fluxes is closely related to the representation of boundarylayer turbulence and cumulus clouds, as well as to the triggering criteria of the deep convection scheme, which will give the onset of precipitation, and its closure, which in turn determines its intensity and duration. Associated clouds will have a different radiative impact given their timing of occurrence within the day. Thus, the evaluation of the representation of the diurnal cycle of precipitation and radiative fluxes gives interesting insights into the progress achieved in the design and setup of physical parameterizations.

This diagnostic evaluates the representation of the diurnal cycle of precipitation over specific regions in the Tropics. The diurnal cycle is known to be different over land and ocean. Over land, the semi-arid Sahel region is contrasted with humid regions (Amazonia) as well as with the two monsoon regions West-Africa and India. Over the ocean, the diagnostic focuses on the Gulf of Guinea, the Indian Ocean and the East and West Equatorial Pacific. A harmonic analysis is done to provide global maps of the timing and amplitude of maximum rainfall with 3-hourly TRMM observations used as a reference.

Optionally, the analysis can be extended to include the diurnal cycle of radiative fluxes at the top of the atmosphere and at the surface (LW and SW, total and clear-sky components). 3-hourly CERES data are used as a reference derived from measurements at the top of the atmosphere and computed using a radiative transfer model at the surface.

\section{Available Namelists and Diagnostics}

Namelists are stored in $\mathrm{nml} /$

- namelist_DiurnalCycle_box_pr.xml

- namelist_DiurnalCycle_box_SFCflux.xml

- namelist_DiurnalCycle_box_TOAflux.xml

- namelist_DiurnalCycle_harmonic.xml

Diagnostics are stored in diag_scripts/

The following diagnostic script is called by namelist_DiurnalCycle_harmonic.xml:

- DiurnalCycle_precip_harmonic.ncl: computes the amplitude, phase and percentage of the variance of the first harmonic of the mean diurnal cycle of precipitation by season over the globe. $3-\mathrm{hr}$ mean precipitation outputs are used and interpolated onto a common $2.5^{\circ} \times 2.5^{\circ}$ grid.

The following diagnostic script is called by namelist_DiurnalCycle_box_pr.xml, namelist_DiurnalCycle_box_SFCflux.xml, namelist_DiurnalCycle_box_TOAflux.xml:

- DiurnalCycle_box.ncl: computes the mean diurnal cycle of precipitation and available radiative fluxes over specific regions and seasons. Regions include Sahel (Sahel, $12^{\circ} \mathrm{N}-23^{\circ} \mathrm{N}, 10^{\circ} \mathrm{E}-10^{\circ} \mathrm{W}$ ), West-Africa (WestAf, $4^{\circ} \mathrm{N}-10^{\circ} \mathrm{N}, 8^{\circ} \mathrm{E}-8^{\circ} \mathrm{W}$ ), Gulf of Guinea (GoG, $2^{\circ} \mathrm{S}-4^{\circ} \mathrm{N}, 8^{\circ} \mathrm{E}-8 \mathrm{~W}$ ), Amazonia (Amazon, $15^{\circ} \mathrm{S}-0^{\circ}, 70^{\circ} \mathrm{W}-50^{\circ} \mathrm{W}$ ), India (India, $12^{\circ} \mathrm{N}-20^{\circ} \mathrm{N}, 75^{\circ} \mathrm{E}-80^{\circ} \mathrm{E}$ ), Indian Ocean (IO, $10^{\circ} \mathrm{S}-$ 
$5^{\circ} \mathrm{N}, 70^{\circ} \mathrm{E}-90^{\circ} \mathrm{E}$ ), East-Equatorial Pacific (EEP, $0^{\circ}-10^{\circ} \mathrm{N}, 110^{\circ} \mathrm{W}-90^{\circ} \mathrm{W}$ ) and West-Equatorial Pacific (WEP, $0^{\circ}-20^{\circ} \mathrm{N}, 130^{\circ} \mathrm{E}-150^{\circ} \mathrm{E}$ ). Seasons include DJF, MAM, JJA and SON.

\section{User settings}

User setting files (cfg files) are stored in $\mathrm{nml} / \mathrm{cfg} \_$DiurnalCycle/

(1) namelist_DiurnalCycle_harmonic.xml

Required diag_script_info attributes

o season: season (DJF, MAM, JJA, SON)

o destgrid: common grid for interpolation ("2.5×2.5")

o latrange_basic: latitude range of box

o lonrange_basic: longitude range of box

o cn_levels_amplitude_basic: contour levels for amplitude plots

o cn_levels_phase_basic: contour levels for phase plots

o cn_levels_var_basic: contour levels for percentage of variance

o styleset: "CMIP5", "DEFAULT"

o my_region: label for region

(2) namelist_DiurnalCycle_box_pr.xml, namelist_DiurnalCycle_box_SFCflux.xml, namelist_DiurnalCycle_box_TOAflux.xml

Required diag_script_info attributes

o season: season (DJF, MAM, JJA, SON)

o latrange: latitude range of box

o lonrange: longitude range of box

o styleset: "CMIP5", "DEFAULT"

o box: label for region

o multi_model_mean: calculate multi-model mean ("y", "n")

o supporting_gridlines: display supporting gridline ("y", "n")

o x_gridlines: display gridline along x-axis ("y", "n")

o y_gridlines: display gridline along y-axis ("y", "n")

\section{Variables}

- $\operatorname{pr}$ (atmos, 3hr mean, longitude latitude time)

- rsds (atmos, $3 \mathrm{hr}$ mean, longitude latitude time)

- $\quad$ rsdscs (atmos, $3 \mathrm{hr}$ mean, longitude latitude time)

- $\quad$ rlds (atmos, $3 \mathrm{hr}$ mean, longitude latitude time) 
- rldscs (atmos, 3hr mean, longitude latitude time)

- $\quad$ rsus (atmos, $3 \mathrm{hr}$ mean, longitude latitude time)

- $\quad$ rsuscs (atmos, $3 \mathrm{hr}$ mean, longitude latitude time)

- rlus (atmos, $3 \mathrm{hr}$ mean, longitude latitude time)

- rlut (atmos, $3 \mathrm{hr}$ mean, longitude latitude time)

- rlutcs (atmos, $3 \mathrm{hr}$ mean, longitude latitude time)

- $\quad$ rsut (atmos, $3 \mathrm{hr}$ mean, longitude latitude time)

- $\quad$ rsutcs (atmos, 3hr mean, longitude latitude time)

\section{Observations and Reformat Scripts}

Note: (1) obs4mips data can be used directly without any preprocessing; (2) see headers of reformat scripts for non-obs4mips data for download instructions.

- TRMM-3B42, 3-hr, $0.25^{\circ} \times 0.25^{\circ}$ : pr (obs4mips)

- CERES-SYN1deg, $3-\mathrm{hr}, 1^{\circ} \times 1^{\circ}$ : rsds, rsdscs, rlds, rldscs, rsus, rsuscs, rlus, rsut, rsutcs, rlut, rlutcs Reformat scripts: reformat_scripts/obs/reformat_obs_ceres-synoptic-sfc.bash, reformat_scripts/obs/reformat_obs_ceres-synoptic-toa.bash

\section{References}

None. 


\section{Example Plots}

JJA-amplitude of the 1st harmonic of the mean diurnal cycle of Precipitation Rate
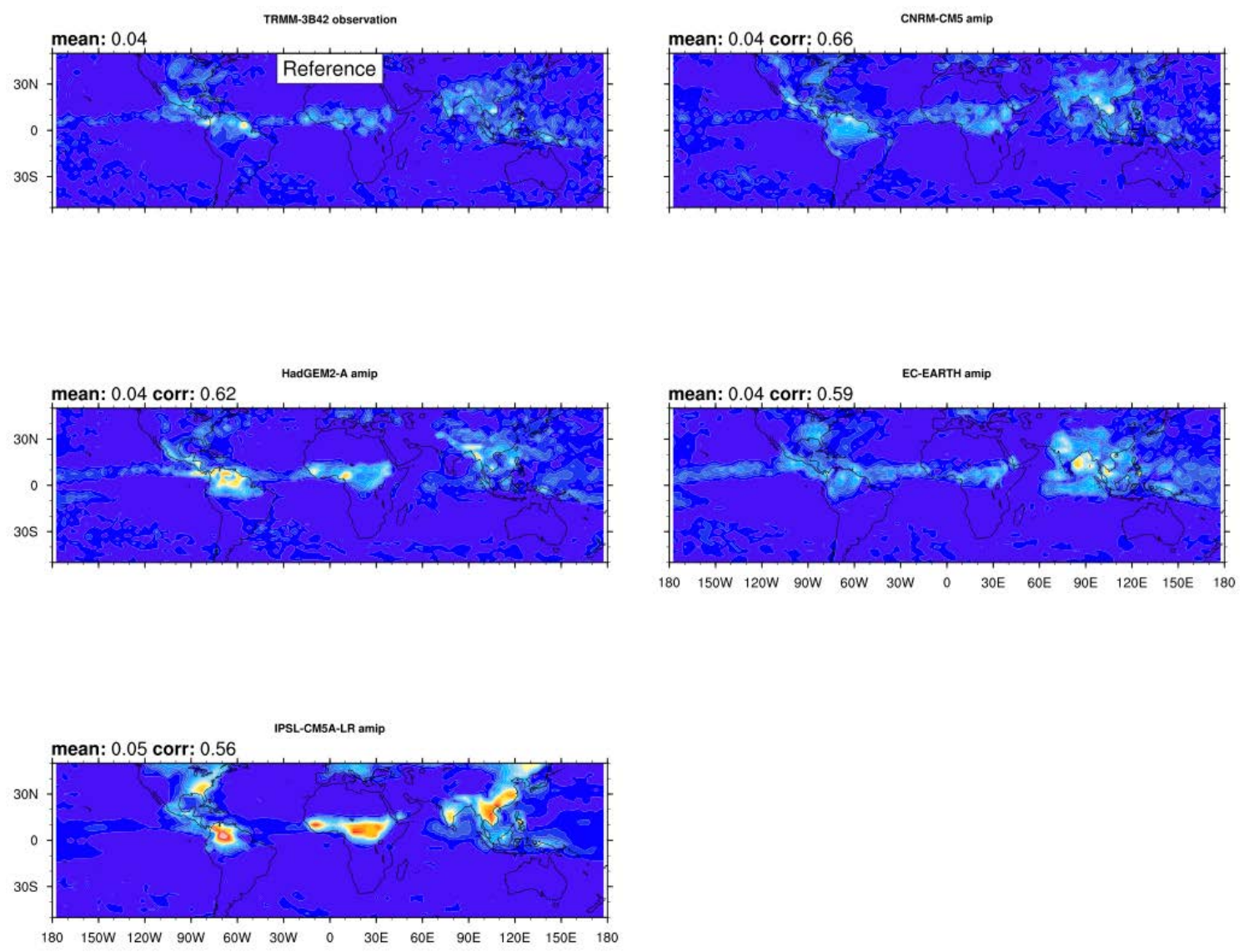

$\begin{array}{llllllllllllllllllllllll}0.00 & 0.03 & 0.06 & 0.09 & 0.12 & 0.15 & 0.18 & 0.21 & 0.24 & 0.27 & 0.30 & 0.33 & 0.36 & 0.39 & 0.42 & 0.45 & 0.48 & 0.51 & 0.54 & 0.57 & 0.60 & 0.63 & 0.66 & 0.69\end{array}$ $\mathrm{mm} /$ hour 
JJA-phase of the 1st harmonic of the mean diurnal cycle of Precipitation Rate
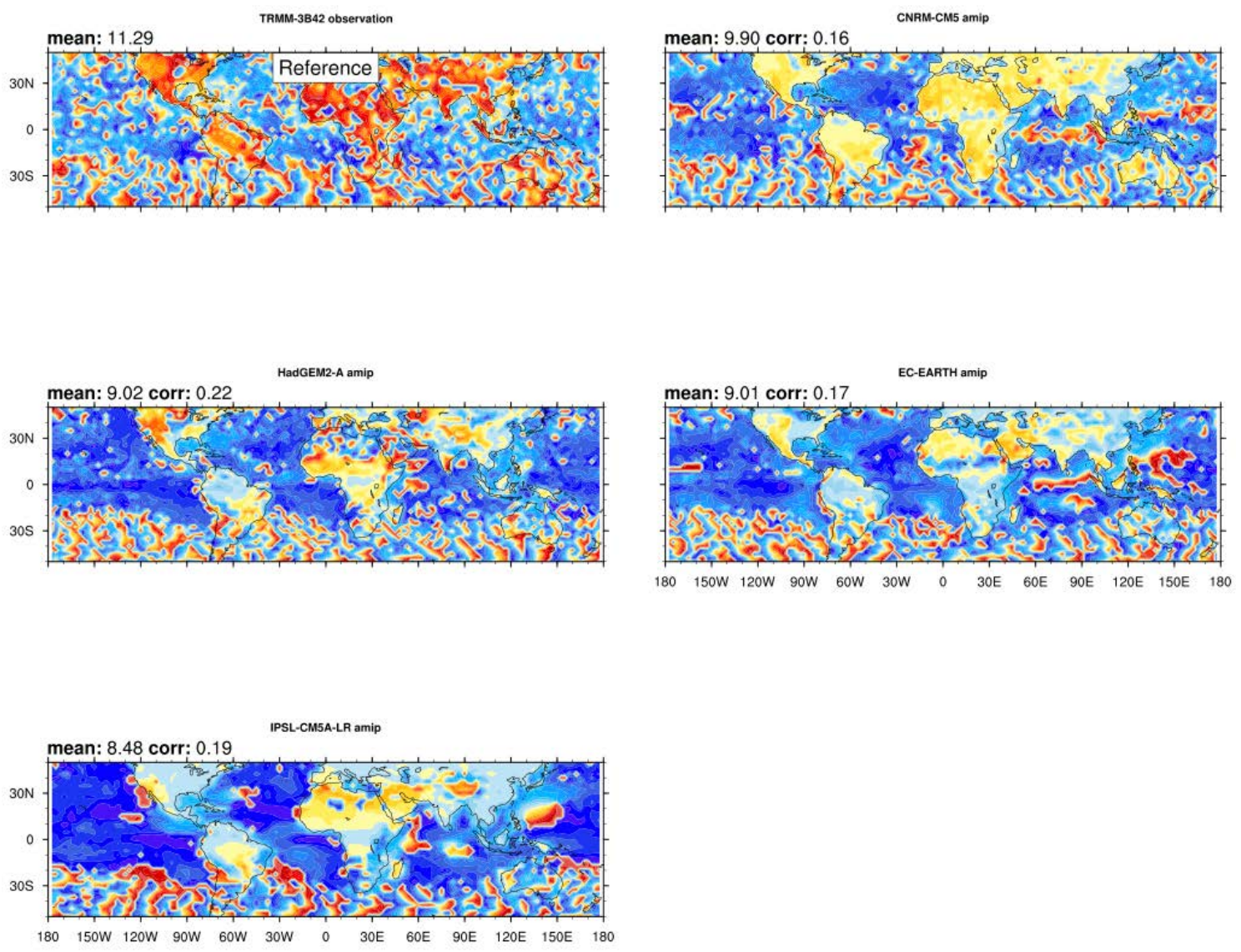

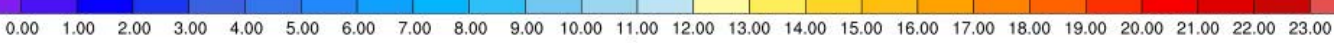
hour 
JJA-var of the 1st harmonic of the mean diurnal cycle of Precipitation Rate
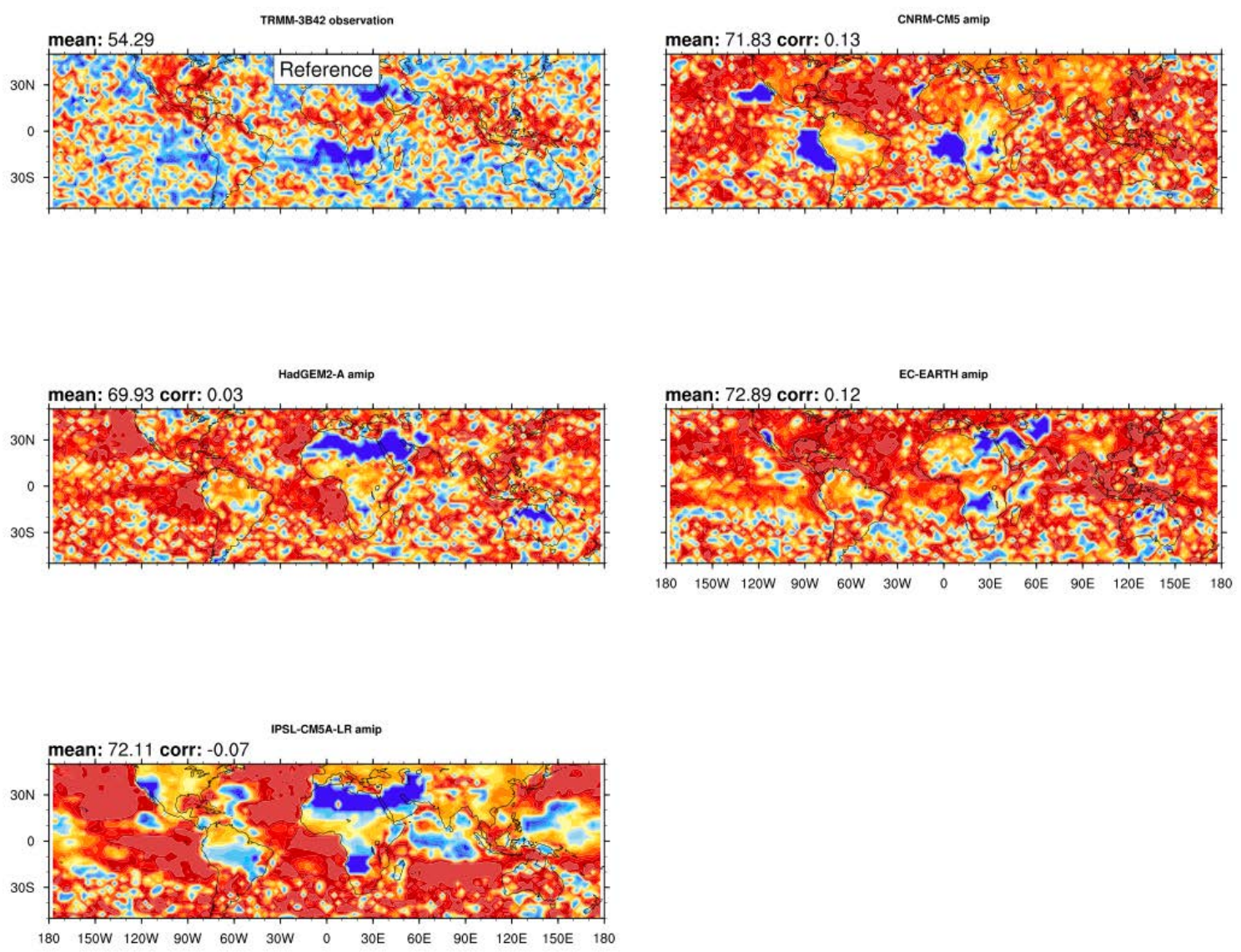

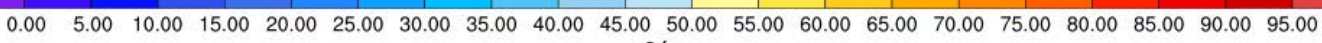
$\%$

DJF Diurnal cycle over Amazonia

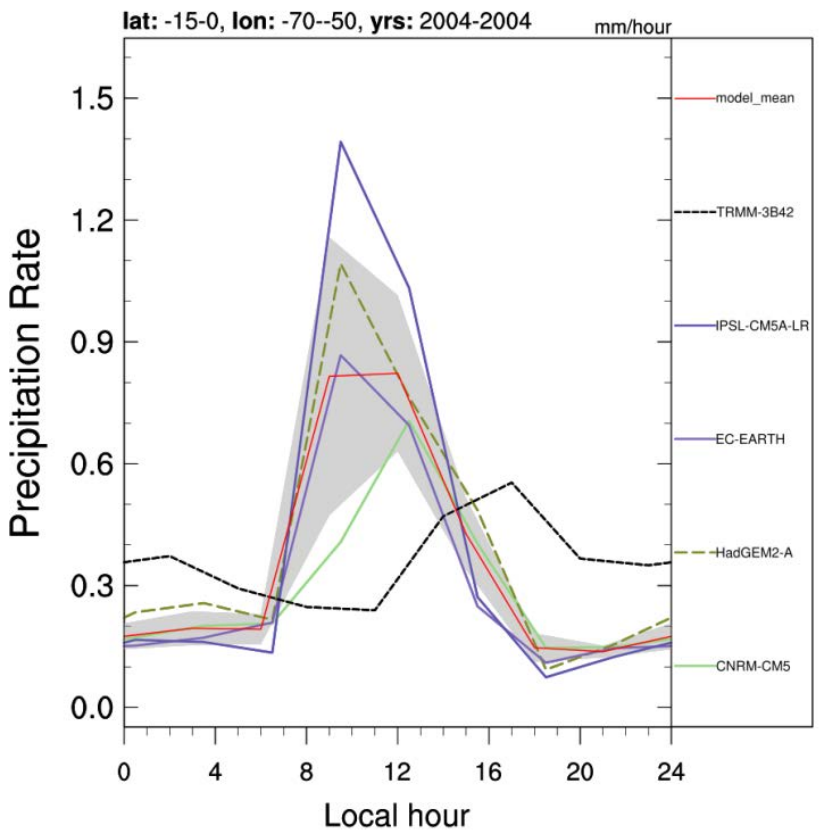

DJF Diurnal cycle over Amazonia

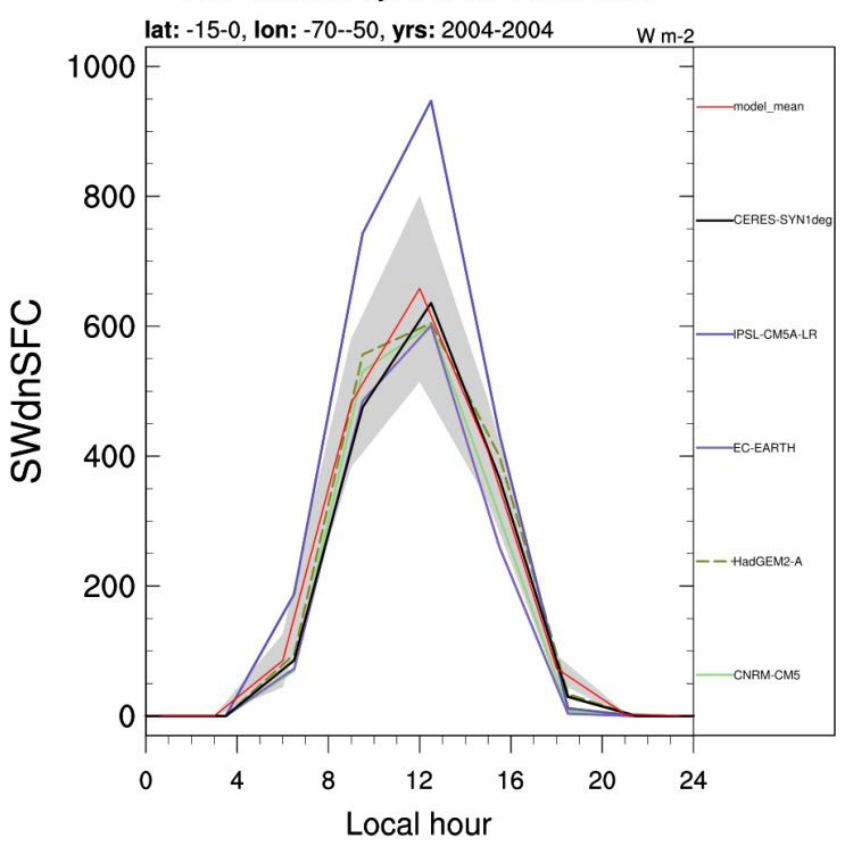




\section{Emergent constraints on carbon cycle feedbacks}

\section{Overview}

Figures from Wenzel et al. (2014) are reproduced with namelist_wenz14jgr.xml. Variables relevant for the carbon cycle-climate feedback such as near surface air temperature (tas), net biosphere productivity (nbp) and (fgco2) are analyzed for coupled (1pctCO2; here the carbon cycle is fully coupled to the climate response) and uncoupled (esmFixCLim1; here the carbon cycle is uncoupled to the climate response) simulations. The standard namelist includes a comparison of cumulated nbp from coupled and uncoupled simulations and includes a set of routines to diagnose the long-term carbon cycle-climate feedback parameter $\left(\mathrm{Gamma}_{\mathrm{LT}}\right)$ from an ensemble of CMIP5 models. Also included in the namelist is a comparison of the interannual variability of nbp and fgco2 for historical simulations used to diagnose the observable sensitivity of $\mathrm{CO}_{2}$ to tropical temperature changes $\left(\mathrm{Gamma}_{\mathrm{IAV}}\right)$. As a key figure of this namelist, the diagnosed values from the models Gamma $\mathrm{LT}_{\mathrm{LT}}$ vs. Gamma $\mathrm{IAV}_{\mathrm{IAV}}$ are compared in a scatter plot constituting an emergent constraint.

\section{Available Namelists and Diagnostics}

Namelists are stored in $\mathrm{nml} /$

- namelist_wenzel14jgr.xml

Diagnostics are stored in diag_scripts/

- tsline.ncl: time line plots of annual means for spatial averages

- carbon_corr_2var.ncl: scatter plot of annual mean anomalies of two different variables; diagnosing and saving Gamma $\mathrm{IAV}_{\mathrm{I}}$

- carbon_constraint.ncl: scatter plot of Gamma $a_{\mathrm{LT}}$ vs. $\mathrm{Gamma}_{\mathrm{IAV}}+$ line plot of probability density functions; diagnosing Gamma

\section{User settings}

User setting files (cfg files) are stored in $\mathrm{nml} / \mathrm{cfg}$ _carbon/

(1) tsline (cfg_carbon_line.ncl, cfg_carbon_line_h.ncl)

Required diag_script_info attributes

o styleset: "CMIP5"

0 ts_minlat: minimum latitude for area-averaging

0 ts_maxlat: maximum latitude for area-averaging

0 ts_minlon: minimum longitude for area-averaging

o ts_maxlon: maximum longitude for area-averaging

o multi_model_mean: True for multi-model mean calculation 
0 ts_maxyear: last year (time range)

0 ts_minyear: first year (time range)

o time_avg: "yearly" (currently, only yearly is available)

0 ts_anomaly: calculates anomalies with respect to the first 10-year average (anom, noanom)

o area_opper: type of area operation (average, sum)

(2) ts_line (cfg_carbon_line_h.ncl)

Required diag_script_info attributes

0 ts_detrend: detrend time series (detr, nodetr)

(3) create_co2_flux.ncl, carbon_corr_2var.ncl

Required diag_script_info attributes

o styleset: "CMIP5"

o con_latrange: 2-element array of latitudes for time series plots if 2D or 3D

o con_lonrange: 2-element array of pressure levels for time series plots if $2 \mathrm{D}$ or 3D

o con_units: label string for units, e.g., "GtC/K"

o pcBGC: label for CMIP5 experiment (for creating filename mask), e.g., "esmFixClim1"

o reg_models: models to be included in emergent constraint (n-element array or (/"all"/)); note: has to be consistent with the list of models specified in the namelist!

\section{Variables}

- $\quad$ tas (atmos, monthly mean, longitude latitude time)

- $\quad \operatorname{co} 2$ (atmos, monthly mean, plev longitude latitude time)

- $\operatorname{nbp}$ (land, monthly mean, longitude latitude time)

- fgco2 (ocean, monthly mean, longitude latitude time)

\section{Observations and Reformat Scripts}

Note: (1) obs4mips data can be used directly without any preprocessing; (2) see headers of reformat scripts for non-obs4mips data for download instructions.

- GCP - Global Carbon Budget including land (nbp) and ocean (fgco2) carbon fluxes

Reformat script: reformat_scripts/obs/reformat_obs_GCP.ncl

- NCEP - National Centers for Environmental Prediction reanalysis data for near surface temperature

Reformat script: reformat_scripts/obs/reformat_obs_NCEP.ncl 


\section{References}

Cox, P. M., D. B. Pearson, B. B. Booth, P. Friedlingstein, C. C. Huntingford, C. D. B. Jones, and C. M. Luke, 2013, Sensitivity of tropical carbon to climate change constrained by carbon dioxide variability, Nature, 494(7437), 341-344. doi: 10.1038/nature11882

Wenzel, S., P. M. Cox, V. Eyring, and P. Friedlingstein, 2014, Emergent Constraints on Climate Carbon Cycle Feedbacks in the CMIP5 Earth System Models, JGR Biogeoscience, 119(5), doi: 2013JG002591. 


\section{Example Plots}

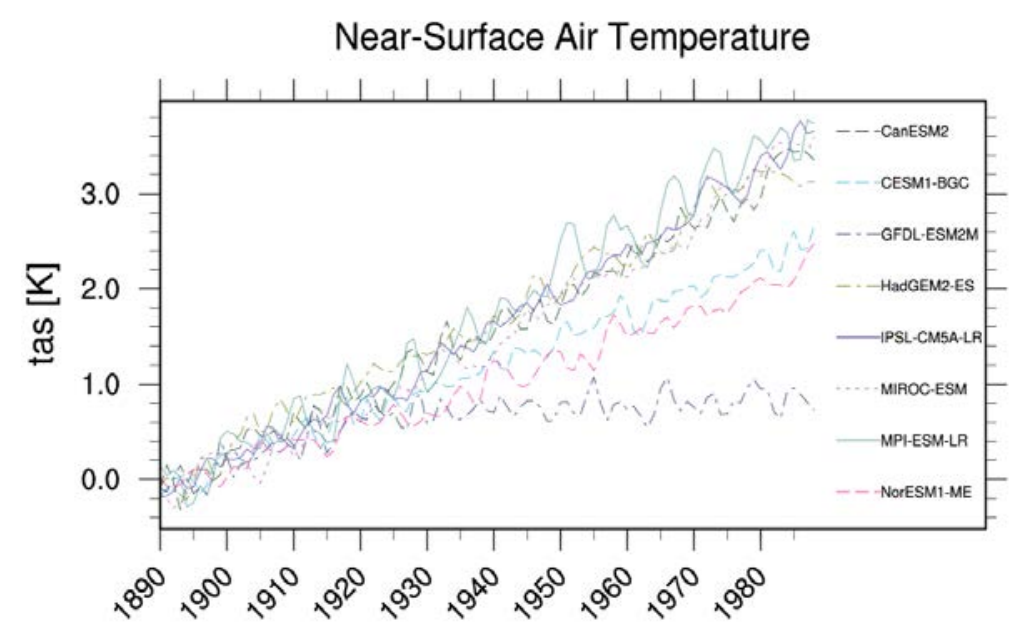

Figure 9 Time series of tropical $\left(30^{\circ} \mathrm{S}-30^{\circ} \mathrm{N}\right)$ mean near surface temperature (tas) change between year 30 and year 110 for the CMIP5 models simulated with prescribed $\mathrm{CO}_{2}\left(1 \% / \mathrm{yr} \mathrm{CO}_{2}\right.$ increase) coupled simulation (1pctCO2).
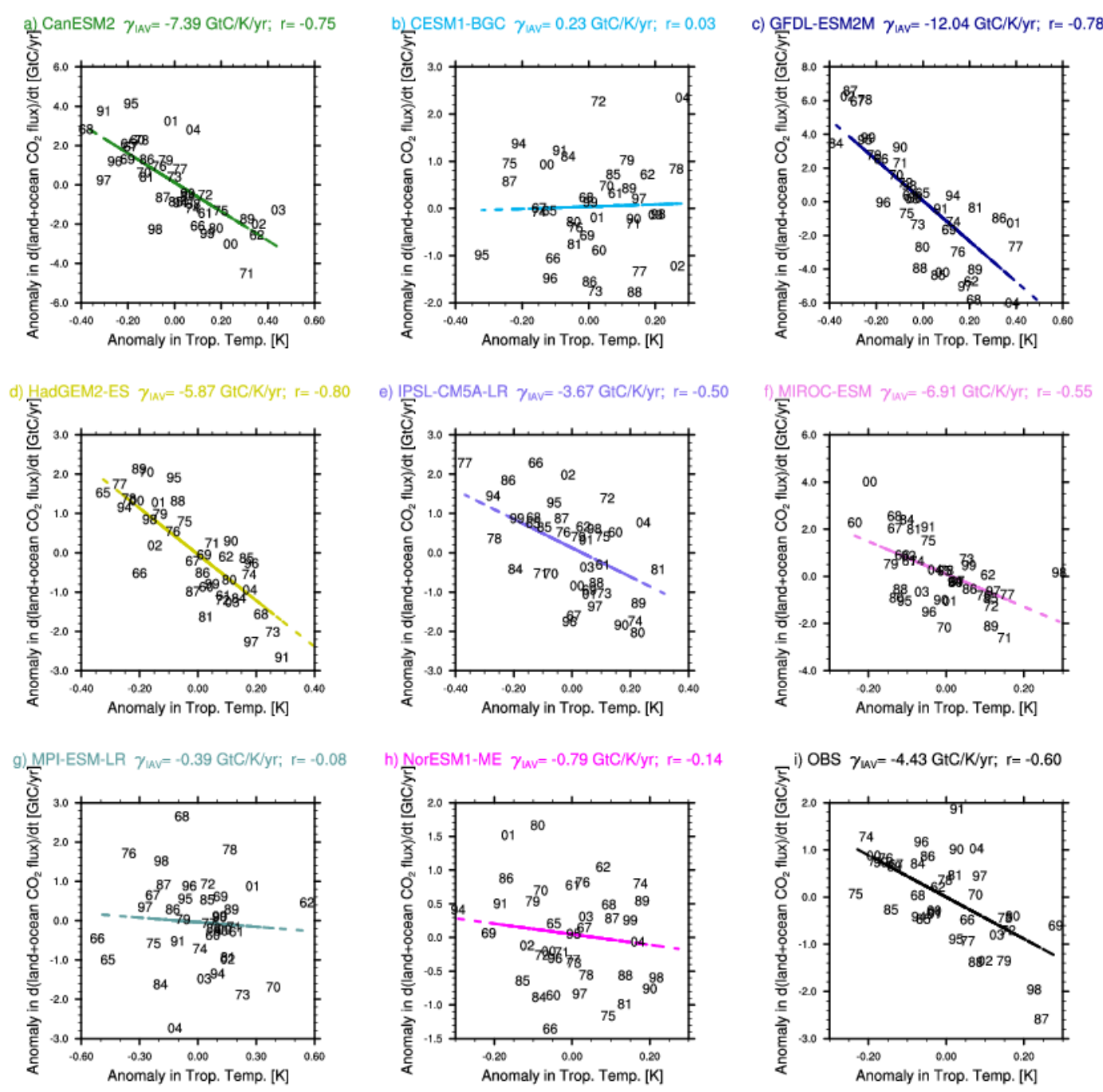

Figure 10 Correlations between the interannual variability of global co2flux (nbp+fgco2) and tropical temperature for the individual CMIP5 models using esmHistorical simulations, and for observations. 

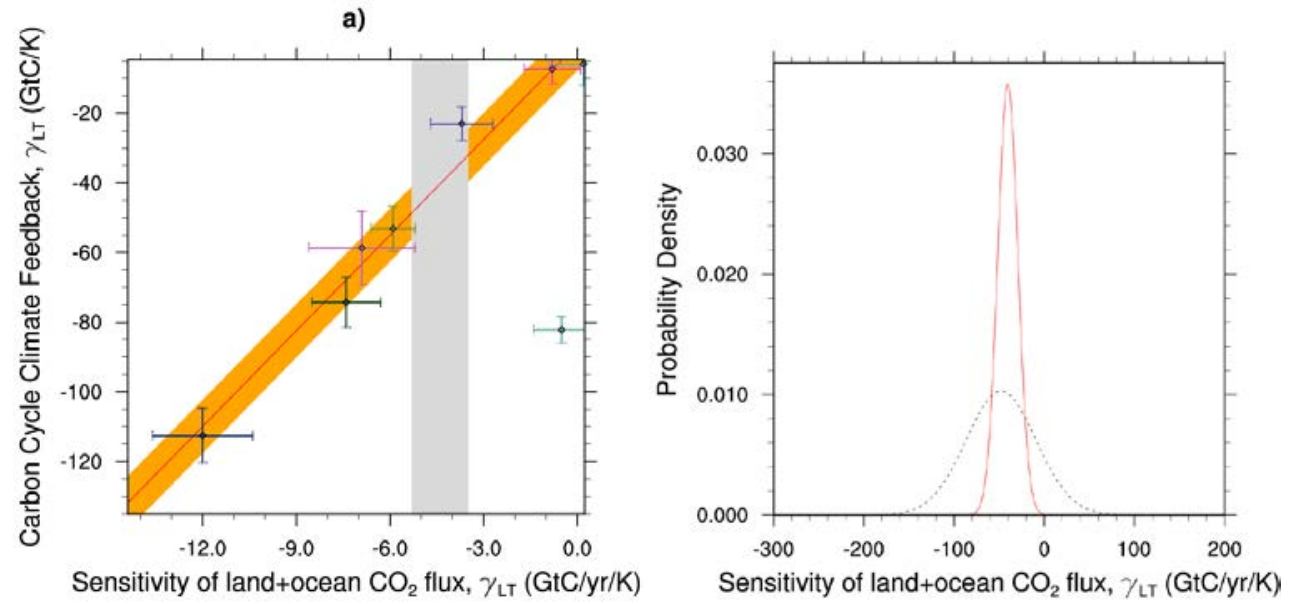

Figure 11 (a) Carbon cycle-climate feedback of tropical land carbon vs. the sensitivity of co2flux to interannual temperature variability in the tropics $\left(30^{\circ} \mathrm{S}-30^{\circ} \mathrm{N}\right)$. The red line shows the linear best fit of the regression together with the prediction error (orange shading) and the gray shading shows the observed range. (b) Probability Density Functions for the pure CMIP5 ensemble (black dashed) and after applying the observed constraint to the models (red solid). 


\section{Evapotranspiration}

\section{Overview}

This diagnostic produces monthly climatologies of evapotranspiration on a global scale. The model outputs are compared to the LandFlux-EVAL data set (Mueller et al., 2013), which is used as reference product for evapotranspiration. An example of application of this diagnostic on CMIP5 models is presented in Mueller and Seneviratne (2014).

\section{Available Namelists and Diagnostics}

Namelists are stored in $\mathrm{nml} /$

- namelist_Evapotranspiration.xml

Diagnostics are stored in diag_scripts/

- Evapotranspiration.ncl

\section{User settings}

User setting files (cfg files) are stored in $\mathrm{nml} / \mathrm{cfg} \_$et/

(1) Evapotranspiration.ncl

Required diag_script_info attributes

o et_colormap: filename of color table

o cn_levels_mean_basic: contour levels of absolute values (array)

0 cn_levels_mean_diff_basic: contour levels of differences

0 sftlf_cutoff: threshold for land sea mask $(>50 \rightarrow$ land grid cell)

Color tables

- diag_scripts/aux/Evapotranspiration/absolute_evapotranspiration.rgb

\section{Variables}

- hfls (atmos, monthly mean, longitude latitude time)

\section{Observations and Reformat Scripts}

Note: see also header of reformat script for downloading instructions.

- The LandFlux-EVAL data set (http://www.iac.ethz.ch/groups/seneviratne/research/LandFluxEVAL). The data set is document in Mueller et al (2013). Download data at the above link, then 
follow the instructions in the reformat script available in the repository. The data set is free to use after registration but may not be redistributed.

Reformat script: reformat_scripts/obs/reformat_obs_LandFlux-EVAL.ncl

\section{References}

Mueller, B., and S. I. Seneviratne (2014), Systematic land climate and evapotranspiration biases in CMIP5 simulations, Geophys. Res. Lett., 41, 128-134, doi: 10.1002/2013GL058055.

Mueller, B., Hirschi, M., Jimenez, C., Ciais, P., Dirmeyer, P. A., Dolman, A. J., Fisher, J. B., Jung, M., Ludwig, F., Maignan, F., Miralles, D. G., McCabe, M. F., Reichstein, M., Sheffield, J., Wang, K., Wood, E. F., Zhang, Y., and Seneviratne, S. I. (2013), Benchmark products for land evapotranspiration: LandFlux-EVAL multi-data set synthesis, Hydrol. Earth Syst. Sci., 17, 37073720, doi: 10.5194/hess-17-3707-2013.

\section{Example Plots}

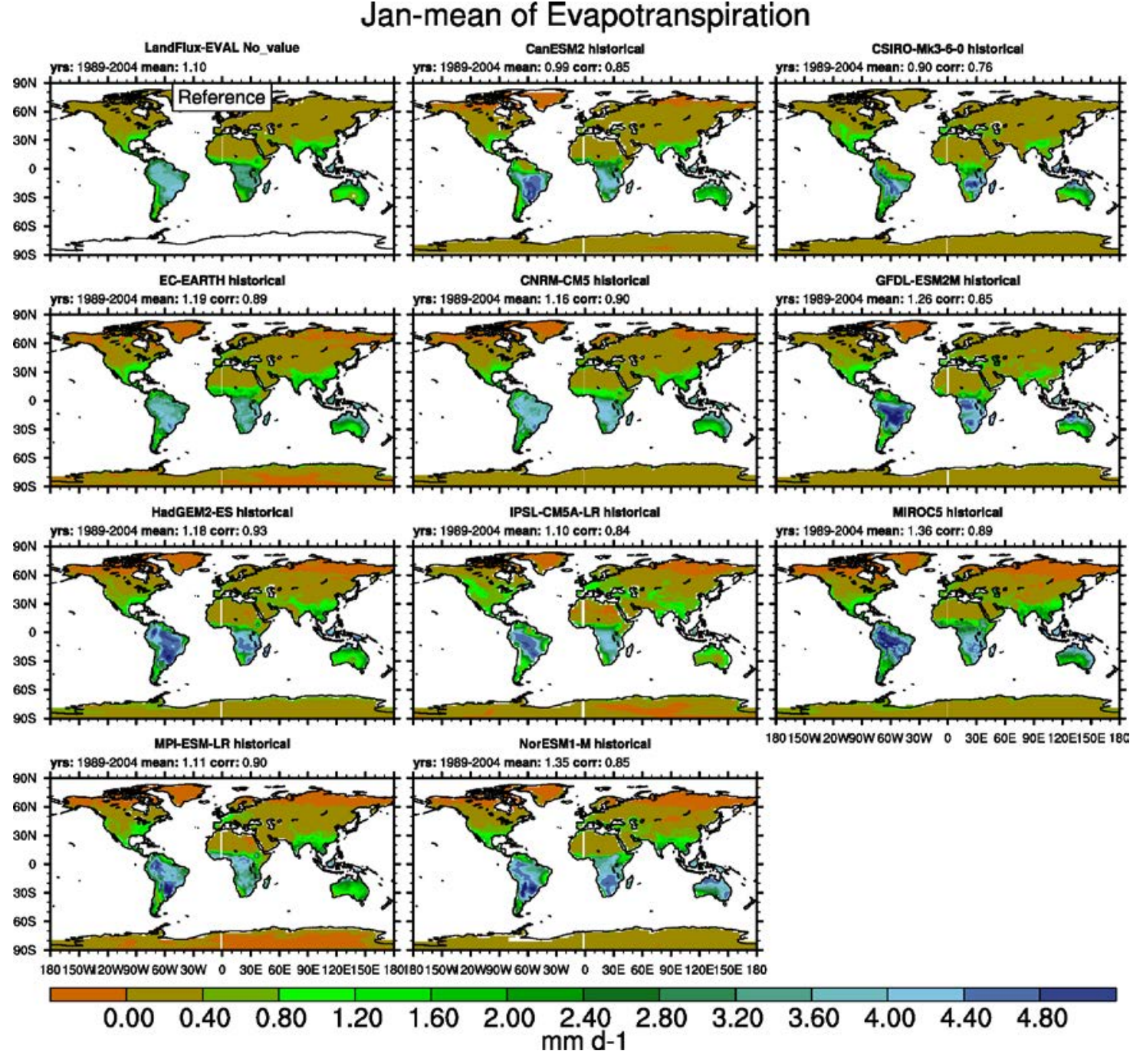


Jan-mean of Evapotranspiration
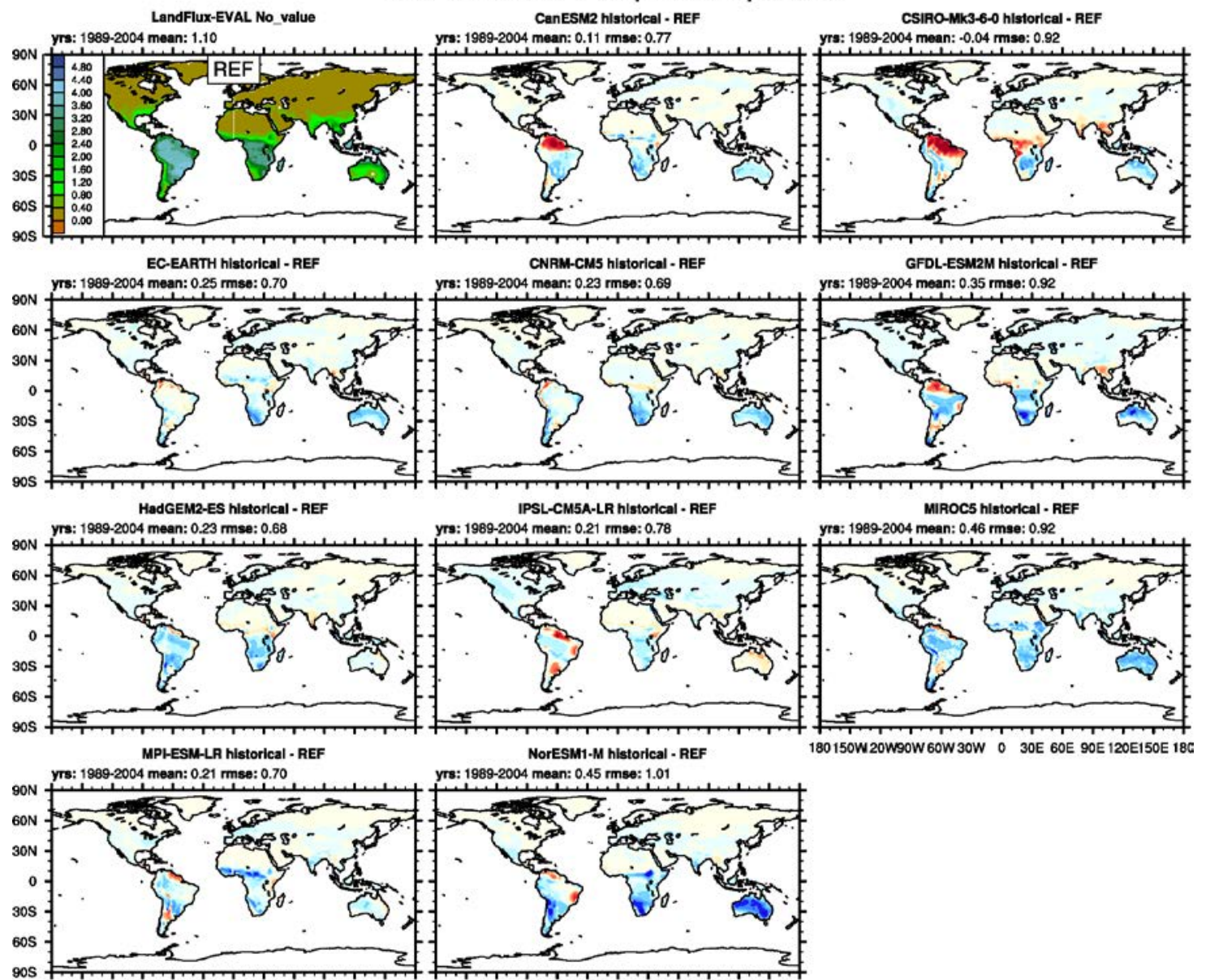

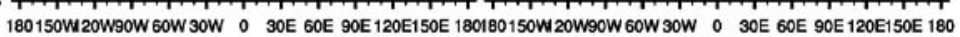

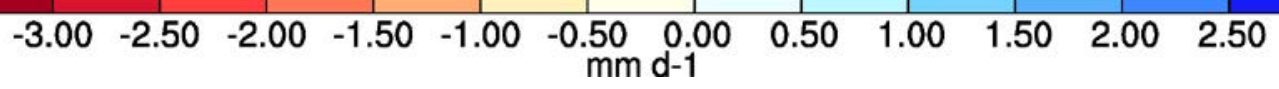


(1) Jan-mean of LandFlux-EVAL obs

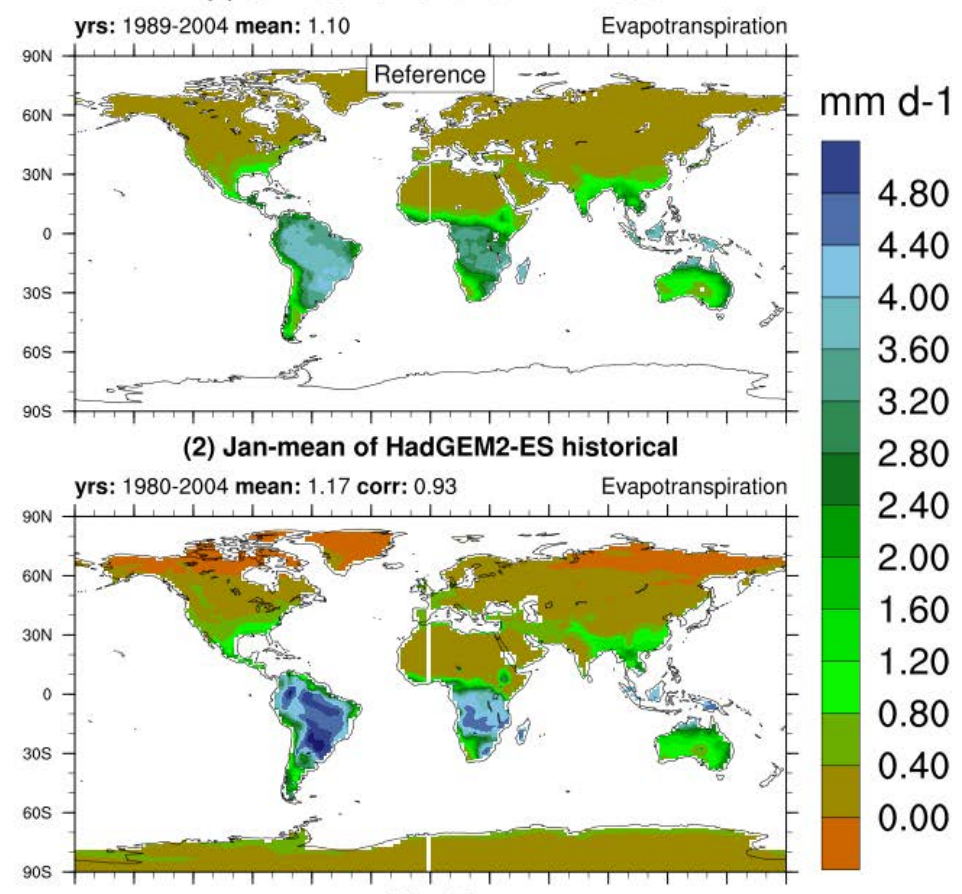

(2) - (1)

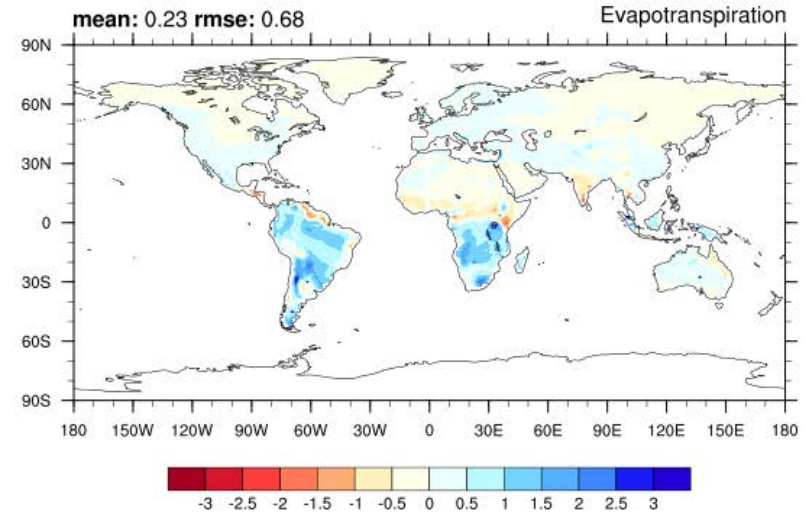




\section{IPCC AR5 chapter 9}

\section{Overview}

The goal of this effort is to code up routines to reproduce Chapter 9 of AR5, so that the plots can be readily reproduced and compared to previous CMIP versions. In this way we can next time start with what was available in the previous round and can focus on developing more innovative methods of analysis rather than constantly having to "re-invent the wheel".

The plots will be done based on a collection of individual namelists. The following figures from Flato et al. (2013) can currently be reproduced:

- Figure 9.2 a,b: Annual-mean surface air temperature for the period 1980-2005. The left panel shows the multi-model mean and the right panel the bias as the difference between the CMIP5 multi-model mean and the climatology from ERA-Interim (Dee et al., 2011).

- Figure 9.4 a,b: Annual-mean precipitation rate $\left(\mathrm{mm} \mathrm{day}^{-1}\right)$ for the period 1980-2005. The left panel shows the multi-model mean and the right panel the bias as the difference between the CMIP5 multimodel mean and the climatology from the Global Precipitation Climatology Project (Adler et al., 2003).

- Figure 9.5: Climatological (1985-2005) annual-mean cloud radiative effects in $\mathrm{Wm}^{-2}$ for the CMIP5 models against CERES EBAF (2001-2011) in $\mathrm{Wm}^{-2}$. Top row shows the shortwave effect; middle row the longwave effect, and bottom row the net effect. Multi-model-mean biases against CERES EBAF 2.6 are shown on the left, whereas the right panels show zonal averages from CERES EBAF 2.6 (black), the individual CMIP5 models (thin gray lines), and the multi-model mean (thick red line).

- Figure 9.7: Relative space-time root-mean square error (RMSE) calculated from the 1980-2005 climatological seasonal cycle of the CMIP5 historical simulations. A relative performance is displayed, with blue shading indicating performance being better and red shading worse, than the median of all model results. A diagonal split of a grid square shows the relative error with respect to the reference data set (lower right triangle) and the alternate data set (upper left triangle). White boxes are used when data is not available for the given model and variable or no alternate data set has been used. The figure shows that performance varies across CMIP5 models and variables, with some models comparing better with observations for one variable and another model performing better for a different variable.

- Figure 9.10: Total column ozone time series for (a) annual global and (b) Antarctic October mean. CMIP5 models are shown in colored lines and the multi-model mean in thick black, their standard deviation as gray shaded area, and observations (NIWA) (black symbols).

- Figure 9.24 a,b: Time series (1960-2005) of (a) September mean Arctic and (b) February mean Antarctic sea ice extent from the CMIP5 historical simulations. The CMIP5 ensemble mean is highlighted in dark red and the individual ensemble members of each model (colored lines) are shown in different line styles. The model results are compared to observations from the NSIDC (1978-2011, black solid line) and the Hadley Centre Sea ice and Sea Surface Temperature (HadISST, 1978-2011, black dashed line). 
- Figure 9.29: Time series of global oceanic mean aerosol optical depth (AOD) from individual CMIP5 models' historical (1850-2005) and RCP 4.5 (2006-2010) simulations compared with MODIS and ESACCI-AEROSOL satellite data.

- Figure 9.32: Monsoon precipitation intensity (upper panels) and monsoon precipitation domain (lower panels) for TRMM and an example of deviations from observations from three CMIP5 models (EC-Earth, HadGEM2-ES, and GFDL-ESM2M).

- Figure 9.45: The carbon cycle-climate feedback $(\gamma \mathrm{LT})$ versus the short-term sensitivity of atmospheric $\mathrm{CO} 2$ to interannual temperature variability $(\gamma \mathrm{IAV})$ in the tropics for CMIP5 models. The red line shows the best fit line across the CMIP5 simulations and the vertical dashed lines show the observed range of $\gamma \mathrm{IAV}$. (b) Probability distribution function (PDF) for $\gamma \mathrm{LT}$. The solid line is derived after applying the interannual variability (IAV) constraint to the models while the dashed line is the prior PDF derived purely from the models before applying the IAV constraint.

\section{Available Namelists and Diagnostics}

Namelists are stored in $\mathrm{nml} /$

- namelist_flato13ipcc.xml

Diagnostics are stored in diag_scripts/

- carbon_constraint.ncl (fig. 9.45: Emergent constraints on carbon cycle feedbacks)

- carbon_corr_2var.ncl (fig. 9.45: Emergent constraints on carbon cycle feedbacks)

- carbon_dummy.ncl (fig. 9.45: Emergent constraints on carbon cycle feedbacks)

- clouds_bias.ncl (figs. 9.2 a,b +9.4 a,b: Clouds)

- clouds_ipcc.ncl (fig. 9.4: Clouds)

- create_co2flux.ncl (fig. 9.45: Emergent constraints on carbon cycle feedbacks)

- eyring13jgr_fig02.ncl (fig. 9.10: Ozone and associated climate impacts)

- perfmetrics_grading.ncl (fig. 9.7: Performance metrics for essential climate parameters)

- perfmetrics_grading_collect.ncl (fig. 9.7: Performance metrics for essential climate parameters)

- perfmetrics_main.ncl (fig. 9.7: Performance metrics for essential climate parameters)

- SAMonsoon_precip_domain.ncl (fig. 9.32: South Asian Summer Monsoon diagnostics)

- SeaIce_tsline.ncl (fig. 9.24 a,b: Sea ice)

- tsline.ncl (fig. 9.29: Aerosol; fig. 9.45: Emergent constraints on carbon cycle feedbacks)

\section{User settings}

See individual diagnostics (Annex C):

- Aerosol

- Clouds 
- Emergent constraints on carbon cycle feedbacks

- Ozone and associated climate impacts

- Performance metrics for essential climate parameters

- Sea ice

- South Asian Summer Monsoon diagnostics

\section{Variables}

- $\quad \operatorname{co} 2$ (atmos, monthly mean, longitude, latitude, plev, time)

- fgco2 (ocean, monthly mean, longitude, latitude, time)

- hus (atmos, monthly mean, longitude, latitude, level, time)

- $\operatorname{nbp}$ (land, monthly mean, longitude, latitude, time)

- od550aer (aero, monthly mean, longitude, latitude, time)

- $\operatorname{pr}$ (atmos, monthly mean, longitude, latitude, time)

- rlut, rlutcs (atmos, monthly mean, longitude, latitude, time)

- rsut, rsutcs (atmos, monthly mean, longitude, latitude, time)

- $\quad$ sic (ocean, monthly mean, longitude, latitude, time)

- ta (atmos, monthly mean, longitude, latitude, level, time)

- tas (atmos, monthly mean, longitude, latitude, time)

- $\quad$ toz (atmos, monthly mean, longitude, latitude, time)

- $\operatorname{tro3}$ (atmos, monthly mean, longitude, latitude, level, time)

- ua (atmos, monthly mean, longitude, latitude, level, time)

- va (atmos, monthly mean, longitude, latitude, level, time)

- $\quad$ zg (atmos, monthly mean, longitude, latitude, level, time)

\section{Observations and Reformat Scripts}

Note: (1) obs4mips data can be used directly without any preprocessing; (2) see headers of reformat scripts for non-obs4mips data for download instructions.

- $\quad$ AIRS L3 (hus - obs4mips)

- CERES-EBAF (rlut, rlutcs, rsut, rsutcs - obs4mips)

- CMAP (pr - reformat_scripts/obs/reformat_obs_CMAP.ncl)

- ERA-Interim (tas, ta, ua, va, zg, hus - reformat_scripts/obs/reformat_obs_ERA-Interim.ncl)

- ESACCI-AEROSOL (od550aer - reformat_scripts/obs/reformat_obs_ESACCI-AEROSOL.ncl) 
- GCP - Global carbon budget including land (nbp) and ocean (fgco2) carbon fluxes (reformat_scripts/obs/reformat_obs_GCP.ncl)

- $\quad$ GPCP-SG (pr - obs4mips)

- HadISST (sic - reformat_scripts/obs/reformat_obs_HadISST.ncl)

- MERRA (pr - reformat_scripts/obs/reformat_obs_MERRA.bash)

- MODIS-L3 (od550aer - obs4mips)

- NCEP (tas, ta, ua, va, zg - reformat_scripts/obs/reformat_obs_NCEP.ncl)

- NIWA (toz - reformat_scripts/obs/reformat_obs_NIWA.ncl)

- NSIDC-NT (sic - reformat_scripts/obs/reformat_obs_NSIDC.ncl)

\section{References}

Adler, R. F., Huffman, G. J., Chang, A., Ferraro, R., Xie, P.-P., Janowiak, J., Rudolf, B., Schneider, U., Curtis, S., Bolvin, D., Gruber, A., Susskind, J., Arkin, P., and Nelkin, E.: The Version-2 Global Precipitation Climatology Project (GPCP) Monthly Precipitation Analysis (1979-Present), J Hydrometeorol, 4, 1147-1167, 2003.

Dee, D. P., Uppala, S. M., Simmons, A. J., Berrisford, P., Poli, P., Kobayashi, S., Andrae, U., Balmaseda, M. A., Balsamo, G., Bauer, P., Bechtold, P., Beljaars, A. C. M., van de Berg, L., Bidlot, J., Bormann, N., Delsol, C., Dragani, R., Fuentes, M., Geer, A. J., Haimberger, L., Healy, S. B., Hersbach, H., Holm, E. V., Isaksen, L., Kallberg, P., Kohler, M., Matricardi, M., McNally, A. P., Monge-Sanz, B. M., Morcrette, J. J., Park, B. K., Peubey, C., de Rosnay, P., Tavolato, C., Thepaut, J. N., and Vitart, F.: The ERA-Interim reanalysis: configuration and performance of the data assimilation system, $\mathrm{Q} J$ Roy Meteor Soc, 137, 553-597, 2011.

Eyring, V., Righi, M., Evaldsson, M., Lauer, A., Wenzel, S., Jones, C., Anav, A., Andrews, O., Cionni, I., Davin, E. L., Deser, C., Ehbrecht, C., Friedlingstein, P., Gleckler, P., Gottschaldt, K.-D., Hagemann, S., Juckes, M., Kindermann, S., Krasting, J., Kunert, D., Levine, R., Loew, A., Mäkelä, J., Martin, G., Mason, E., Phillips, A., Read, S., Rio, C., Roehrig, R., Senftleben, D., Sterl, A., van Ulft, L. H., Walton, J., Wang, S., and Williams, K. D.: ESMValTool (v1.0) - a community diagnostic and performance metrics tool for routine evaluation of Earth System Models in CMIP, Geosci. Model Dev. Discuss., 8, 7541-7661, doi: 10.5194/gmdd-8-7541-2015, 2015.

Flato, G., J. Marotzke, B. Abiodun, P. Braconnot, S.C. Chou, W. Collins, P. Cox, F. Driouech, S. Emori, V. Eyring, C. Forest, P. Gleckler, E. Guilyardi, C. Jakob, V. Kattsov, C. Reason and M. Rummukainen, 2013: Evaluation of Climate Models. In: Climate Change 2013: The Physical Science Basis. Contribution of Working Group I to the Fifth Assessment Report of the Intergovernmental Panel on Climate Change [Stocker, T.F., D. Qin, G.-K. Plattner, M. Tignor, S.K. Allen, J. Boschung, A. Nauels, Y. Xia, V. Bex and P.M. Midgley (eds.)]. Cambridge University Press, Cambridge, United Kingdom and New York, NY, USA. 


\section{Example Plots}
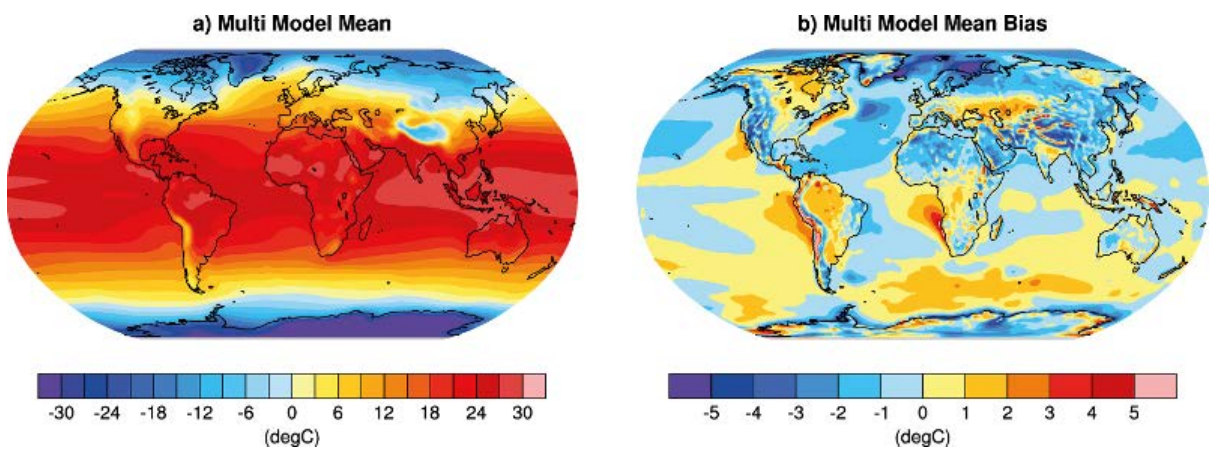

Figure 12 Resembling Flato et al. (2013), Fig. 9.2a,b (Eyring et al., 2015: Fig. 2).
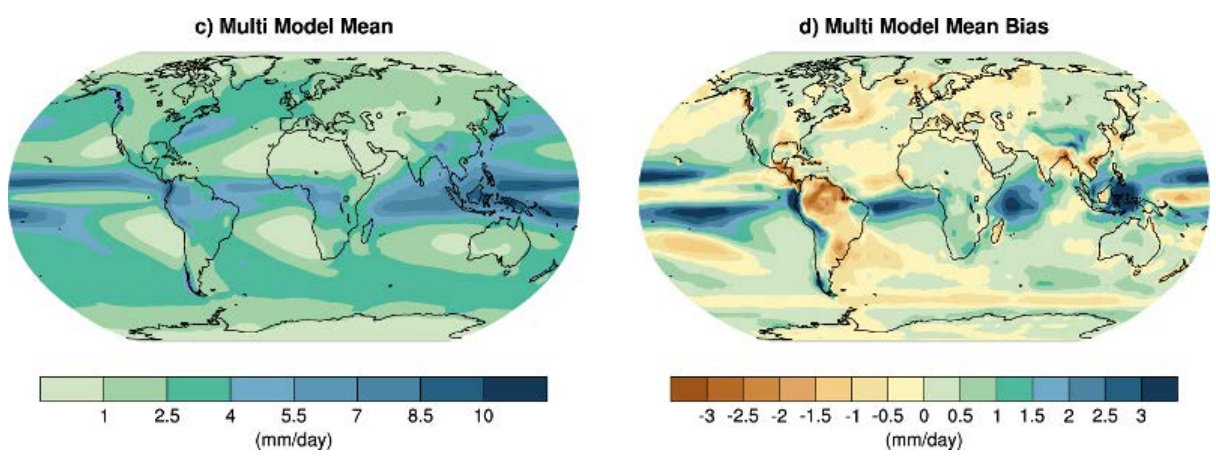

Figure 13 Resembling Flato et al. (2013), Fig. 9.4a,b (Eyring et al., 2015: Fig. 2). 

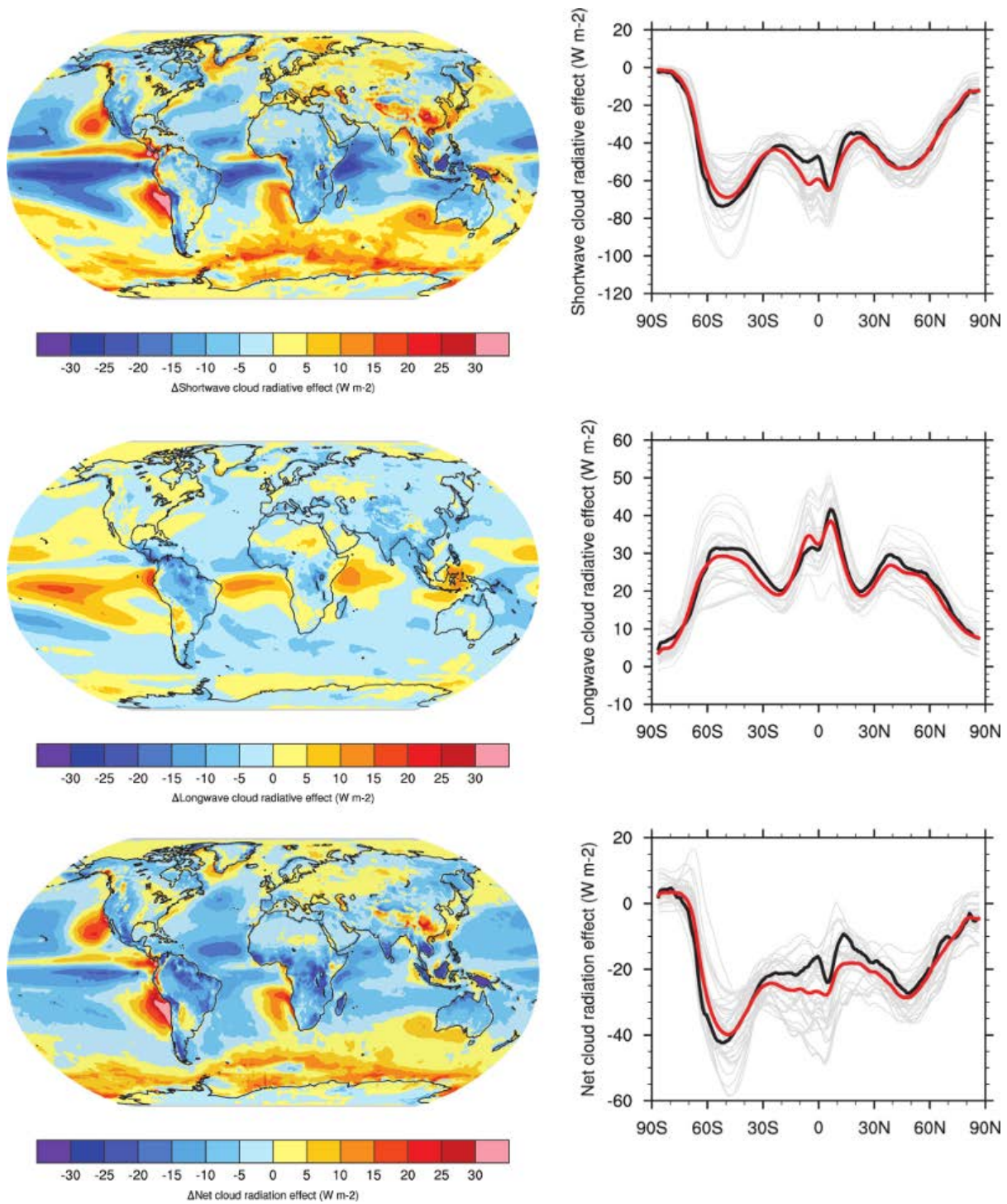

Figure 14 Resembling Flato et al. (2013), Fig. 9.5 (Eyring et al., 2015: Fig. 12). 


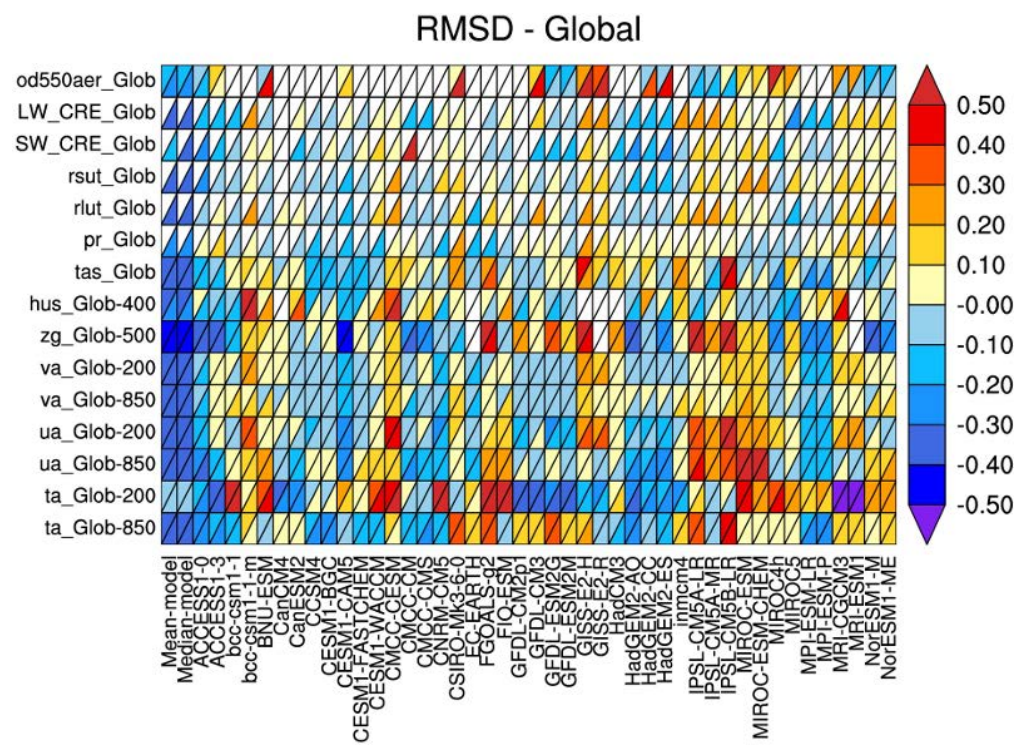

Figure 15 Resembling Flato et al. (2013), Fig. 9.7 (Eyring et al., 2015: Fig. 2).
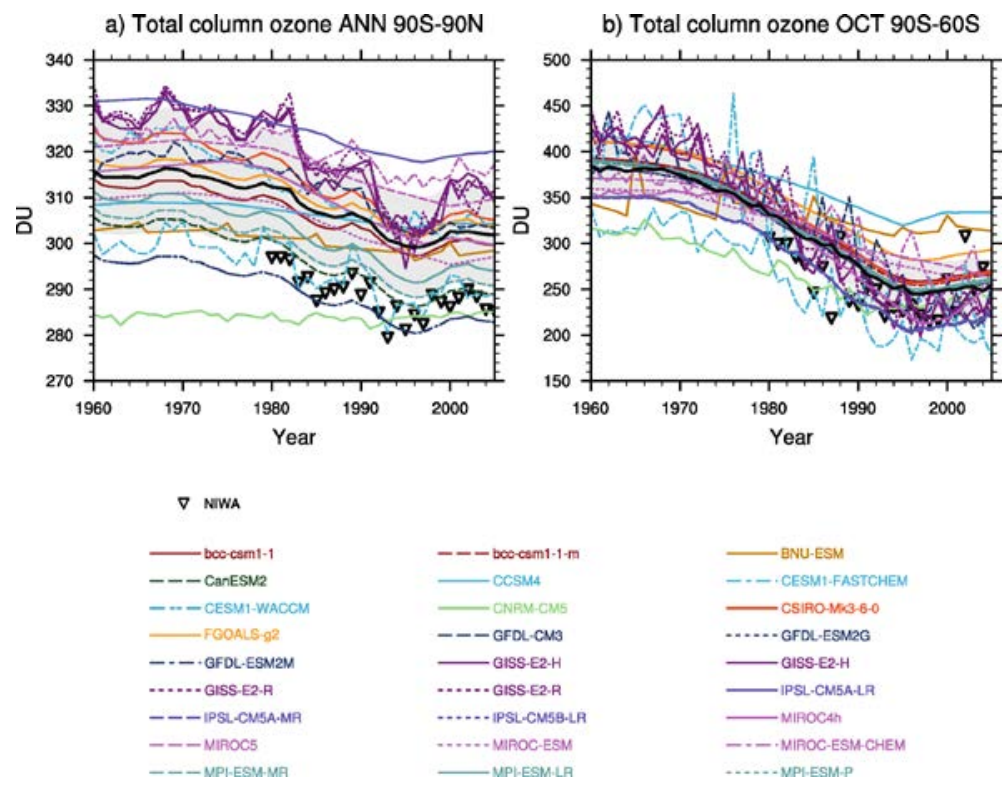

Figure 16 Resembling Flato et al. (2013), Fig. 9.10 (Eyring et al., 2015: Fig. 25).

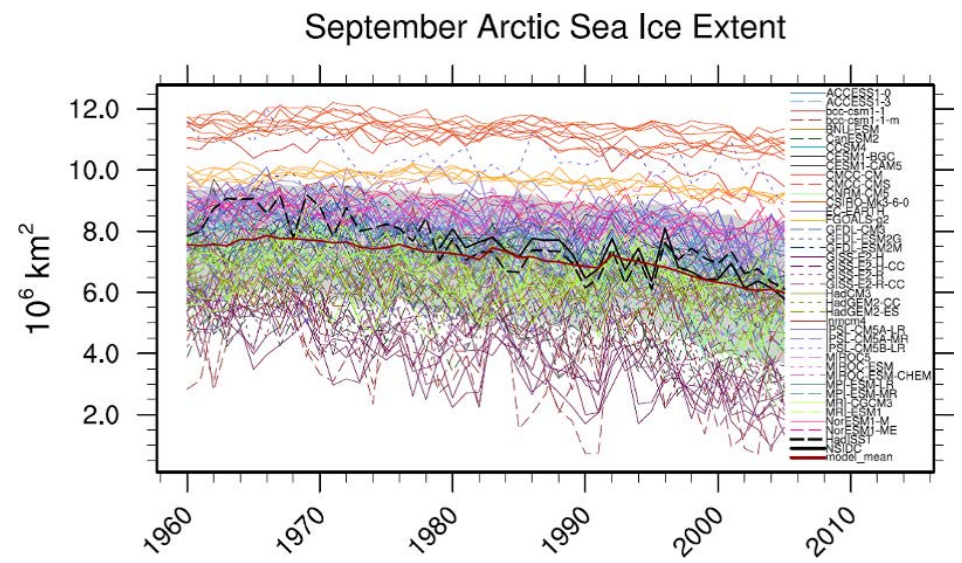

Figure 17 Resembling Flato et al. (2013), Fig. 9.10 (Eyring et al., 2015: Fig. 25). 
Ambient Aerosol Optical Thickness at $550 \mathrm{~nm}$

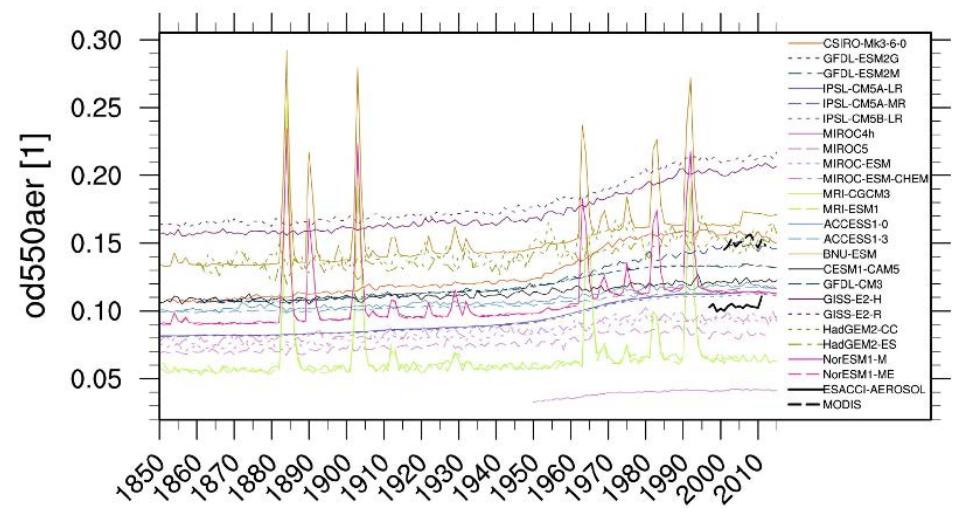

Figure 18 Resembling Flato et al. (2013), Fig. 9.24 (Eyring et al., 2015: Fig. 17).

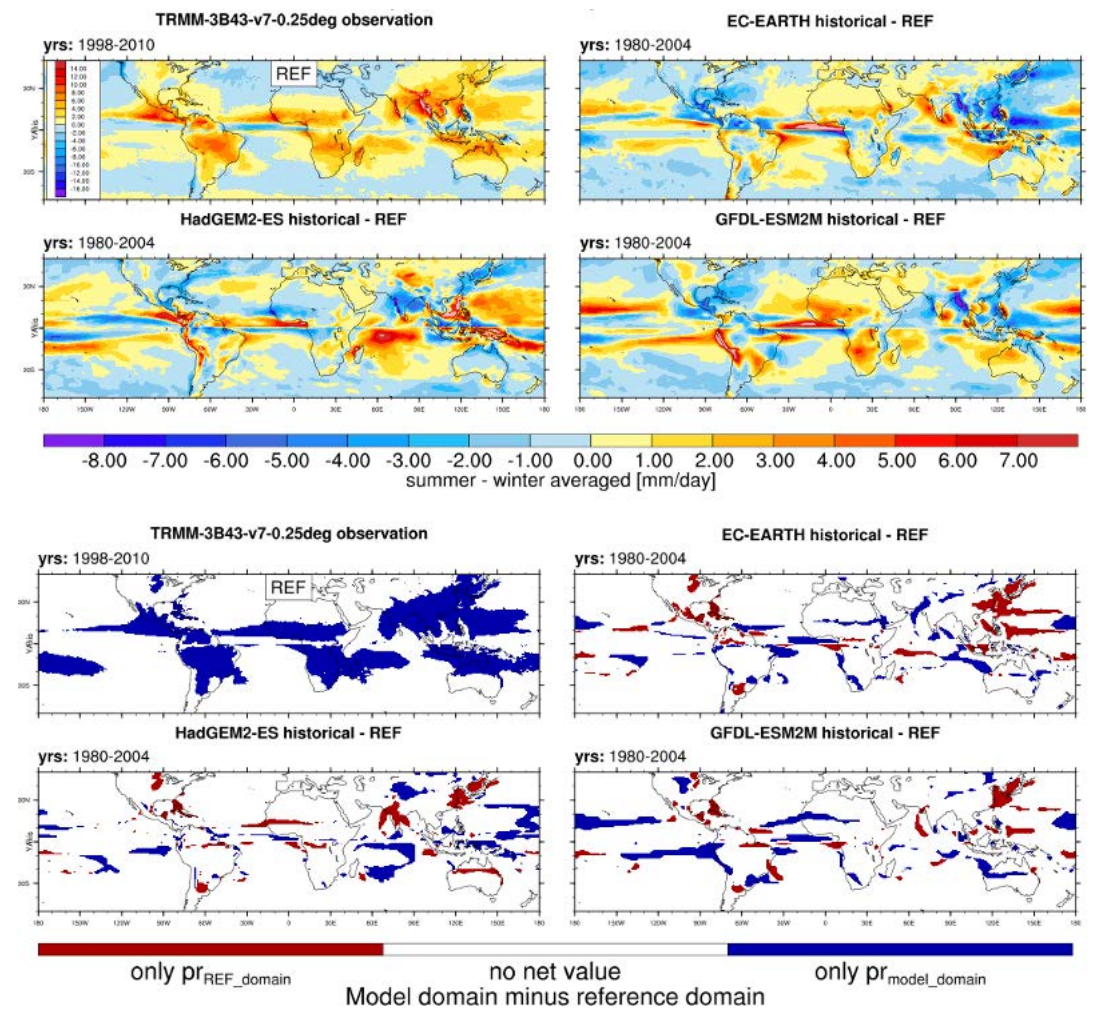

Figure 19 Resembling Flato et al. (2013), Fig. 9.32 (Eyring et al., 2015: Fig. 5).

a)

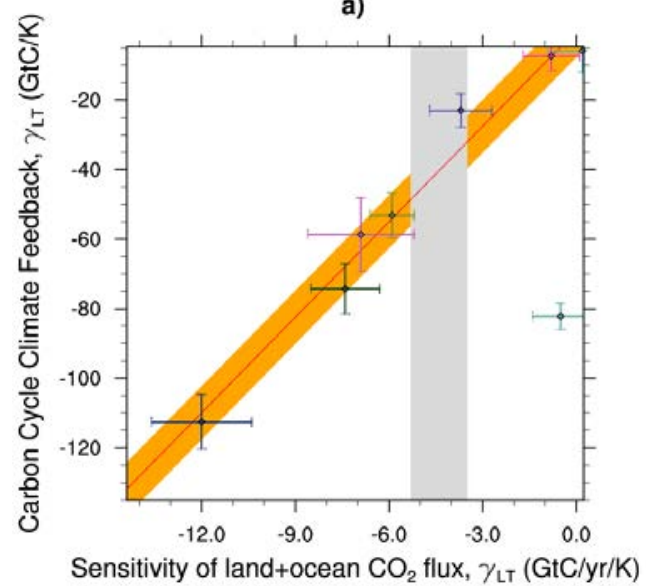

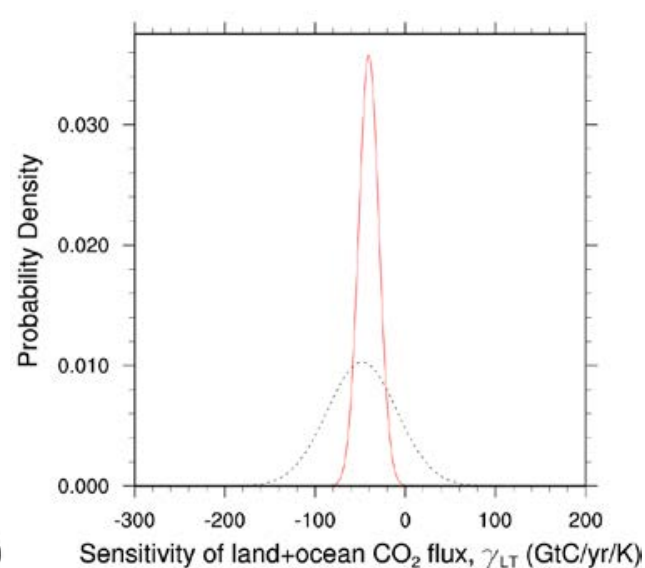

Figure 20 Resembling Flato et al. (2013), Fig. 9.45 (Eyring et al., 2015: Fig. 26). 


\section{Land and ocean components of the global carbon cycle}

\section{Overview}

This namelist reproduces most of the figures of Anav et al. (2013):

- seasonal cycle plot for different regions

- errorbar plot for different regions showing mean and standard deviation

- scatterplot for different regions showing mean vs. interannual variability

- 3D-scatterplot for different regions showing mean vs. linear trend and the model variability index (MVI) as a third dimension (color coded)

- scatterplot for different regions comparing two variable against each other (cSoil vs. cVeg)

In addition, performance metrics are calculated for all variables using the performance metric diagnostics (see details in section "Performance metrics for essential climate parameters"). This however applies only to variables on a regular grid (i.e., not to fgco2), as irregular grids are not yet supported by the performance "mwtr".

\section{Available Namelists and Diagnostics}

Namelists are stored in $\mathrm{nml} /$

- namelist_anav13jclim.xml

Diagnostics are stored in diag_scripts/

- CarbonCycle_main.ncl: calculate temporal and spatial averages and plots the variable as error-bar and seasonal cycle plots.

- CarbonCycle_MVI.ncl: calculate the model variability index (MVI), interannual variability (IAV) and mean, and draw them in a 3D scatter plot.

- CarbonCycle_2vars.ncl: draw a scatter plot with two variables.

- (perfmetrics_main.ncl): see section "Performance metrics for essential climate parameters"

- (perfmetrics_grading.ncl): see section "Performance metrics for essential climate parameters"

\section{User settings}

User setting files (cfg files) are stored in $\mathrm{nml} / \mathrm{cfg} \_$anav13jclim/

(1) CarbonCycle_MVI.ncl

Diag_script_info attributes

- grid: target grid for regridding ("0.5deg", "1deg", "2deg") 
- region: region for spatial average ("Global", "Northern Hemisphere", "Southern Hemisphere", "Tropics")

- ref_model : reference model, e.g. "CRU"

(2) CarbonCycle_main.ncl

Diag_script_info attributes

- region: region for spatial average ("Global", "Northern Hemisphere", "Southern Hemisphere", "Tropics")

- legend_outside: plot legend in a separate file (True, False)

• $\quad$ styleset: plot style set ("CMIP5", "EMAC", "DEFAULT")

- $\quad$ sort: sort models alphabetically (True, False)

- seasonal_cycle_plot: create seasonal cycle plot (True, False)

- errorbar_plot: create error bar plot (True, False)

- mean_IAV_plot: create mean (x-axsis), IAV (y-axsis) plot (True, False)

(3) CarbonCycle_2vars.ncl

\section{Diag_script_info attributes}

- region: region for spatial average ("Global", "Northern Hemisphere", "Southern Hemisphere", "Tropics")

- legend_outside: plot legend in a separate file (True, False)

- styleset: plot style set ("CMIP5", "EMAC", "DEFAULT")

(4) perfmetrics_main.ncl, perfmetric_grading.ncl

Diag_script_info attributes

- see section "Performance metrics for essential climate parameters"

\section{Variables}

- $\operatorname{tas}$ (atmos, monthly mean, longitude latitude time)

- $\operatorname{pr}$ (atmos, monthly mean, longitude latitude time)

- $\operatorname{nbp}$ (lmon, monthly mean, longitude latitude time)

- $\quad$ gpp (lmon, monthly mean, longitude latitude time)

- lai (lmon, monthly mean, longitude latitude time)

- $\quad \operatorname{cVeg}$ (lmon, monthly mean, longitude latitude time)

- cSoil (lmon, monthly mean, longitude latitude time) 
- fgco2 (omon, monthly mean, longitude latitude time)

\section{Observations and Reformat Scripts}

Note: (1) obs4mips data can be used directly without any preprocessing; (2) see headers of reformat scripts for non-obs4mips data for download instructions.

- $\quad$ CRU (near-sruface air temperature, precipitation)

Reformat script: reformat_scripts/obs/reformat_obs_CRU.ncl

- MTE (gross primary productivity of carbon)

- LAI3g (leaf area index)

- JMA-TRANSCOM $\left(\mathrm{CO}_{2}\right.$ exchange $)$

- HSWD (soil carbon content)

- NDP (vegetation carbon content)

Reformat scripts: The reformat scripts for the following data sets could not be included in the ESMVaITool v1.0: MTE, LAI3g, JMA-TRANSCOM, HSWD, and NDP. Please contact the author of this diagnostic package (A. Anav) for obtaining the reformat scripts.

\section{References}

Anav, A. et al.: Evaluating the land and ocean components of the global carbon cycle in the CMIP5 Earth System Models, J. Climate, 26, 6901-6843, doi: 10.1175/JCLI-D-12-00417.1, 2013.

\section{Example Plots}
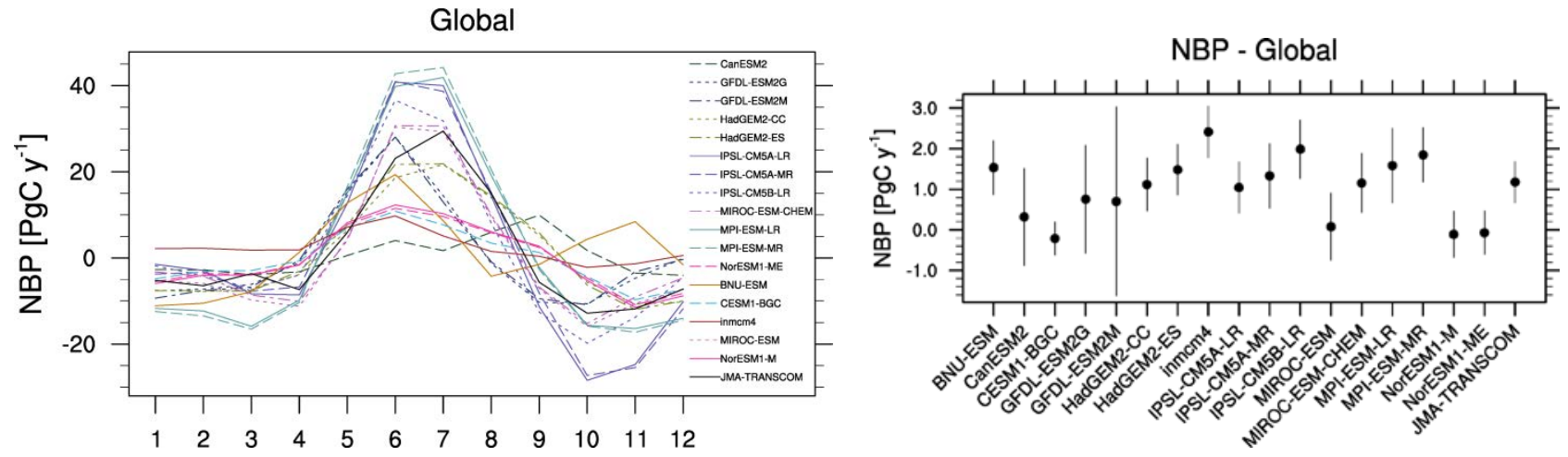

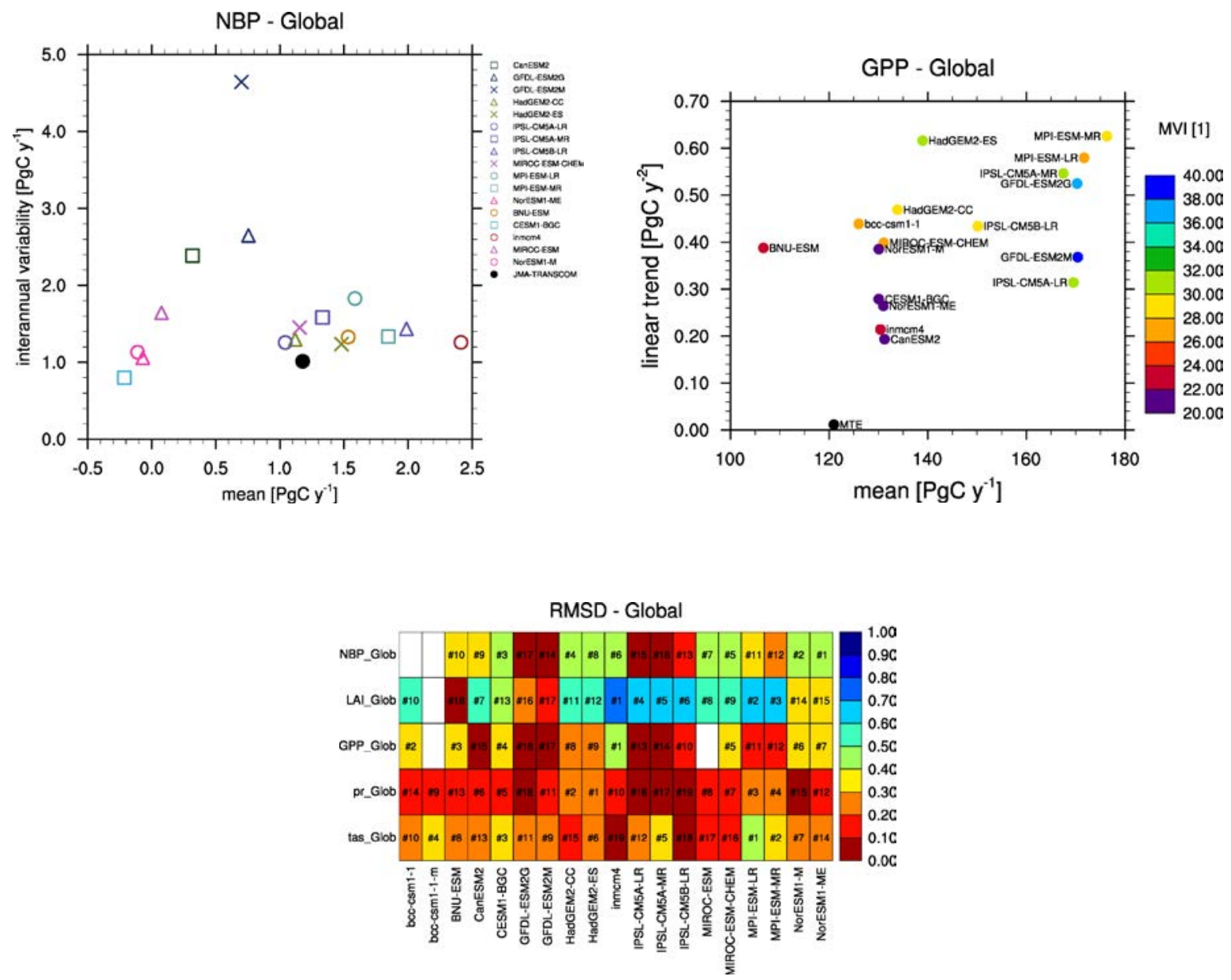


\section{Marine biogeochemistry}

\section{Overview}

A series of routines are provided to support the evaluation of ocean biogeochemical cycles at global scales as simulated by both ocean-only and coupled climate-carbon cycle models. Diagnostics have been implemented to generate time series line and contour plots for climatological distributions and inter-annual or interseasonal (e.g., JJAS) variability of selected ocean biogeochemical variables along with companion plots for differences ("diff") relative to a chosen reference (observational) data set. Basic benchmarking statistics are also provided (bias, standard deviation, root mean squared error, correlation coefficient).

\section{Available Namelists and Diagnostics}

Namelists are stored in $\mathrm{nml} /$

- namelist_GlobalOcean.xml

Diagnostics are stored in diag_scripts/

- GO_comp_map.ncl: this diagnostic takes input from up to four models, one of which is defined as the reference, to produce contour maps of the time-mean (climatology) and standard deviation (interannual or inter-seasonal [e.g., JJAS] variability) together with the difference between the reference and each model. GO_comp_map.ncl is based on SAM_monsoon/SAMonsoon_wind_basic.ncl and uses many SAMonsoon underlying functions. Extensions to these routines include support for the pre-processing and interpolation of curvilinear ocean grids (e.g., ORCA2, NEMO) along with the option to use 3-D (TO3M; depth-resolving) input model data (ocean) and select a single depth level on which to produce comp_maps.

- GO_tsline.ncl: this diagnostic (developed from SeaIce_tsline.ncl) produces annual and seasonal time series plots from monthly ocean data, and includes reformatting options to convert input irregularly gridded data into a version that NCL can interpret by introducing intermediate referencing coordinates in dimensioning the variable, and writing gridcell areas to the same file. Plots can be produced either globally or from a selected latitude-longitude range, with optional extensions including the ability to mask model fields with observational coverage, plot anomaly fields, or overlay derived fields such as linear trend lines, running means or multi-model means. Support for irregular grids (e.g., ORCA2, ORCA1) is provided to, for instance, apply a weighting based on gridcell areas before calculating averages (see reformat_scripts/GO/).

\section{User settings}

User setting files (cfg files) are stored in $\mathrm{nml} / \mathrm{cfg} \_\mathrm{GO} /$

(1) GO_tsline.ncl

$$
\begin{aligned}
& \text { Required diag_script_info attributes } \\
& \text { o month: "A" (A = annual mean) } \\
& \text { o styleset: "CMIP5" (CMIP5, DEFAULT, GO, EXPT) }
\end{aligned}
$$


o colors = (/"red", "green", "blue", "yellow", "purple", "black", "gray", "white", "pink", "orange", "brown", "palegreen"/)

o ts_multimean: "True" = plot multi-model mean \& stddev; "False" = don't

0 ts_latrange: $\min$. and max. latitude for area-averaging of first variable (array)

0 ts_lonrange: $\min$. and max. longitude for area-averaging of first variable (array)

Optional diag_info_script attributes

o ts_anom: calculates anomalies with respect to the first 10-year average (anom, noanom)

o ts_ymin: $y$-axis minimum

o ts_ymax: $y$-axis maximum

o ts_min_year: start year

0 ts_max_year: end year

o ts_smooth: smoothing (True, False)

0 ts_trend: overlay trend line (True, False)

0 ts_coverage: not used

(2) GO_comp_map.ncl

Required diag_script_info attributes

o GO_season: season (e.g., "JJASO")

o GO_detrend: detrend data (True, False)

o latrange_basic: min. and max latitude of region (array)

o lonrange_basic: min. and max. longitude of region (array)

o ref_model: name of reference data set

o cn_levels_mean_basic: contour levels for mean (array)

o cn_levels_mean_diff_basic: contour levels for differences (array)

o cn_levels_stddev_basic: contour levels for standard deviation (array)

o cn_levels_stddev_diff_basic: contour levels for difference in standard deviation (array)

o regrid_for_these_projects: list of project names that will be regridded (e.g., "CMIP5_gridfile", "GO_gridfile", "CMIP5_fx") (array)

\section{Variables}

- $\quad$ spco2 (ocnBgchem, monthly mean, longitude latitude time)

- $\quad c h l$ (ocnBgchem, monthly mean, longitude latitude time)

- talk (ocnBgchem, monthly mean, longitude latitude time)

- o2 (ocnBgchem, monthly mean, longitude latitude olevel time) 


\section{Observations and Reformat Scripts}

Note: (1) obs4mips data can be used directly without any preprocessing; (2) see headers of reformat scripts for non-obs4mips data for download instructions.

- spco2: Surface Ocean CO2 Atlas (SOCAT version 2; Bakker et al., 2014)

Reformat script: reformat_scripts/obs/reformat_obs_SOCAT.csh

- spco2: Two Surface Ocean pCO2 Mapping Intercomparison (SOCOM) products: UEA-SI v1.0 (Jones et al., In Rev.) and ETH-SOM-FFN (Landshüster et al., 2014)

Reformat script: reformat_scripts/obs/reformat_obs_ETH-SOM-FFN.csh

- chl: Chlorophyll concentration data from Sea-viewing WIde Field-of-view Sensor (SeaWIFs; available at: http://oceancolor.gsfc.nasa.gov/SeaWiFS/)

Reformat script: reformat_scripts/obs/reformat_obs_SeaWIFS.csh

- o2: World Ocean Atlas (WOA) 2005 dissolved oxygen concentration data with corrections applied as described in Bianchi et al. (2012)

Reformat script: reformat_scripts/obs/reformat_obs_woa2005.csh

- talk: Monthly surface climatological Total Alkalinity (T14; Takahashi et al., 2014)

Reformat script: reformat_scripts/obs/reformat_obs_takahashi14.csh

\section{References}

Bakker, D. C. E., Pfeil, B., Smith, K., Hankin, S., Olsen, A., Alin, S. R., Cosca, C., Harasawa, S., Kozyr, A., Nojiri, Y., O'Brien, K. M., Schuster, U., Telszewski, M., Tilbrook, B., Wada, C., Akl, J., Barbero, L., Bates, N. R., Boutin, J., Bozec, Y., Cai, W.-J., Castle, R. D., Chavez, F. P., Chen, L., Chierici, M., Currie, K., de Baar, H. J. W., Evans, W., Feely, R. A., Fransson, A., Gao, Z., Hales, B., HardmanMountford, N. J., Hoppema, M., Huang, W.-J., Hunt, C. W., Huss, B., Ichikawa, T., Johannessen, T., Jones, E. M., Jones, S. D., Jutterström, S., Kitidis, V., Körtzinger, A., Landschützer, P., Lauvset, S. K., Lefèvre, N., Manke, A. B., Mathis, J. T., Merlivat, L., Metzl, N., Murata, A., Newberger, T., Omar, A. M., Ono, T., Park, G.-H., Paterson, K., Pierrot, D., Ríos, A. F., Sabine, C. L., Saito, S., Salisbury, J., Sarma, V. V. S. S., Schlitzer, R., Sieger, R., Skjelvan, I., Steinhoff, T., Sullivan, K. F., Sun, H., Sutton, A. J., Suzuki, T., Sweeney, C., Takahashi, T., Tjiputra, J., Tsurushima, N., van Heuven, S. M. A. C., Vandemark, D., Vlahos, P., Wallace, D. W. R., Wanninkhof, R., and Watson, A. J.: An update to the Surface Ocean $\mathrm{CO}_{2}$ Atlas (SOCAT version 2), Earth Syst. Sci. Data, 6, 69-90, doi: 10.5194/essd-6-69-2014, 2014.

Bianchi, D., Dunne, J. P., Sarmiento, J. L., and Galbraith, E. D.: Data-based estimates of suboxia, denitrification, and $\mathrm{N}_{2} \mathrm{O}$ production in the ocean and their sensitivities to dissolved $\mathrm{O}_{2}$, Global Biogeochem. Cy., 26, GB2009, doi: 10.1029/2011GB004209, 2012.

Jones, S. D., Le Quere, C., Rödenbeck, C., Manning, A. C., and Olsen, A.: A statistical gap-filling method to interpolate global monthly surface ocean carbon dioxide data, J, Adv. Model Earth Syst., in review. 
Key, R. M., Kozyr, A., Sabine, C. L., Lee, K., Wanninkhof, R., Bullister, J. L., Feely, R. A., Millero, F. J., Mordy, C., and Peng, T.-H.: A global ocean carbon climatology: results from Global Data Analysis Project (GLODAP), Global Biogeochem. Cy., 18, GB4031, doi: 10.1029/2004GB002247, 2004.

Rödenbeck, C., Keeling, R. F., Bakker, D. C. E., Metzl, N., Olsen, A., Sabine, C., and Heimann, M.: Global surface-ocean $\mathrm{pCO}_{2}$ and sea-air $\mathrm{CO}_{2}$ flux variability from an observation-driven ocean mixed-layer scheme, Ocean Sci., 9, 193-216, doi: 10.5194/os-9-193-2013, 2013.

Takahashi, T., Sutherland, S. C., Chipman, D. W., Goddard, J. G., Ho, C., Newberger, T., Sweeney, C., and Munro, D. R.: Climatological distributions of $\mathrm{pH}, \mathrm{pCO}_{2}$, total $\mathrm{CO}_{2}$, alkalinity, and $\mathrm{CaCO}_{3}$ saturation in the global surface ocean, and temporal changes at selected locations, Mar. Chem., 164, 95-125, doi: 10.1016/j.marchem.2014.06.004, 2014.

\section{Example Plots}

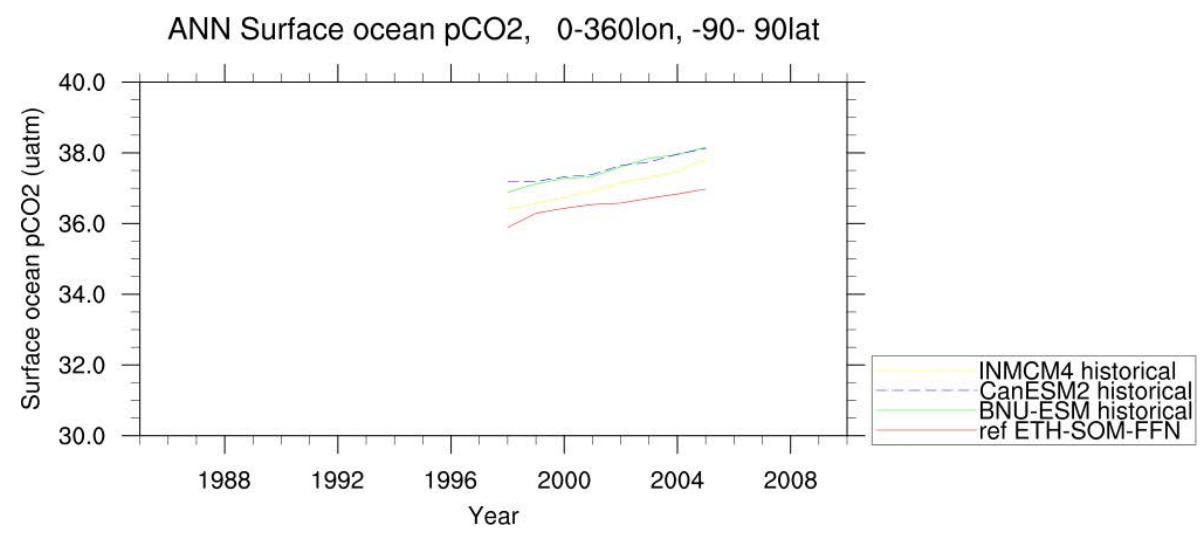

JFM-diff of o2_onelev
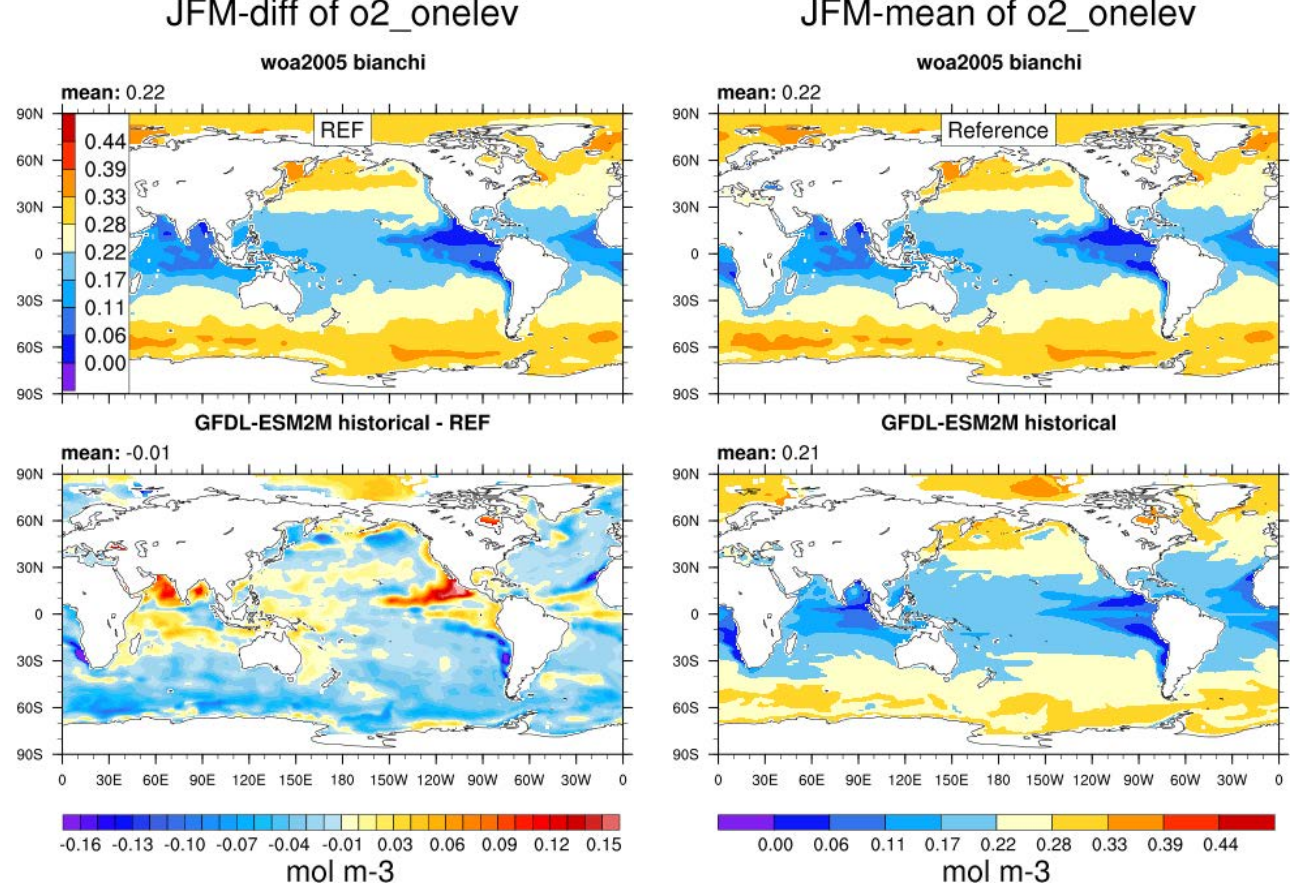
(1) JFMAMJJASOND-mean of ref ETH-SOM-FFN
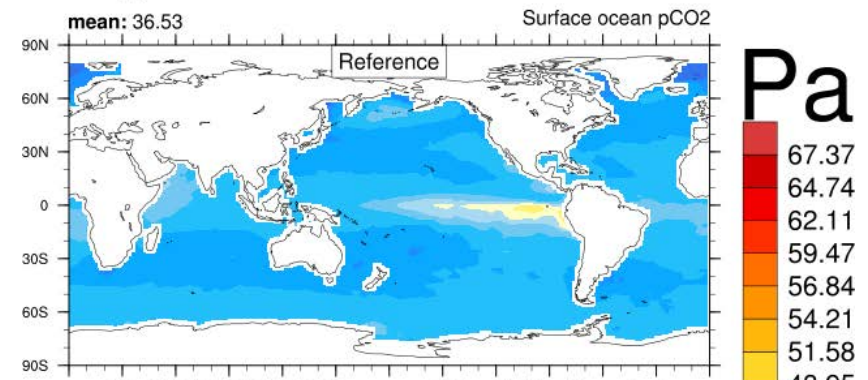

(2) JFMAMJJASOND-mean of CanESM2 historical

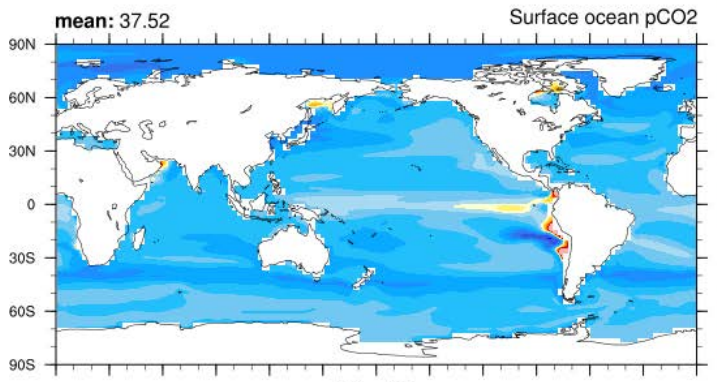

46.32

43.68

41.05

38.42

35.79

33.16

30.53

27.89

25.26

22.63

20.00

(2) - (1)

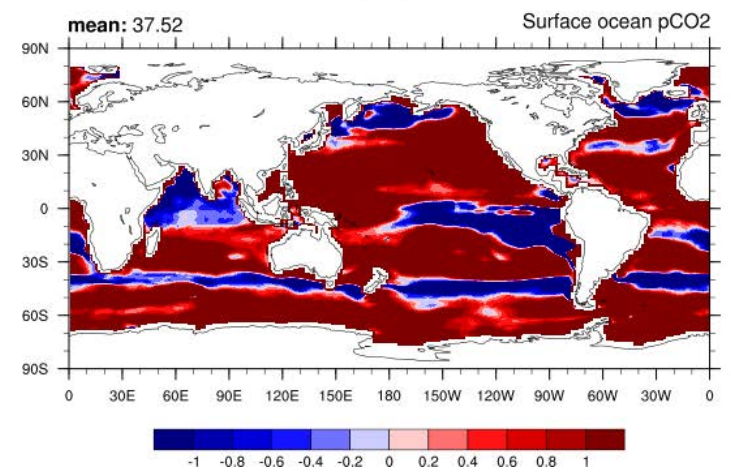

JFMAMJJASOND-mean of Surface ocean pCO2 ref ETH-SOM-FFN
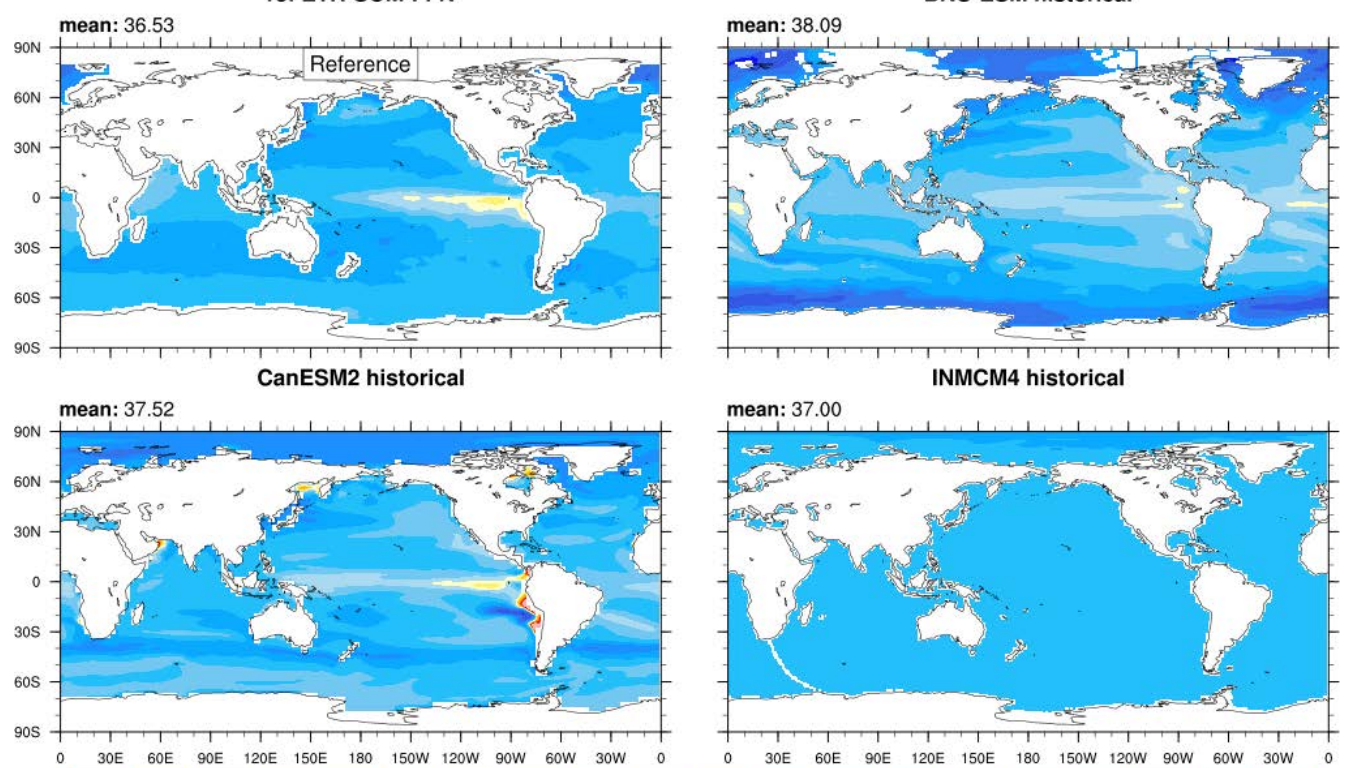

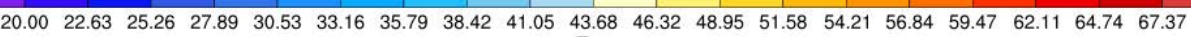




\section{Madden-Julian Oscillation (MJO)}

\section{Overview}

To assess the main MJO features in ESMs, a namelist with a number of diagnostics developed by the US CLIVAR MJO Working Group (Kim et al., 2009; Waliser et al., 2009) has been implemented in the ESMValTool. These diagnostics are calculated using precipitation (pr), outgoing longwave radiation (OLR) (rlut), eastward (ua) and northward wind (va) at $850 \mathrm{hPa}$ (u850) and $200 \mathrm{hPa}$ (u200) against various observations and reanalysis data sets for boreal summer (May-October) and winter (November-April).

Observation and reanalysis data sets include GPCP-1DD for precipitation, ERA-Interim and NCEP-DOE reanalysis 2 for wind components (Kanamitsu et al., 2001) and NOAA polar-orbiting satellite data for OLR (Liebmann and Smith, 1996). The majority of the scripts are based on example scripts available at http://ncl.ucar.edu/Applications/mjoclivar.shtml. Daily data is required for most of the scripts. The basic diagnostics include mean seasonal state and 20-100 day bandpass filtered variance for precipitation and $\mathrm{u} 850$ in summer and winter. To better assess and understand model biases in the MJO, a number of more sophisticated diagnostics have also been implemented. These include: univariate empirical orthogonal function (EOF) analysis for 20-100 day bandpass filtered daily anomalies of precipitation, OLR, u850 and u200. To illustrate the northward and eastward propagation of the MJO, lag-longitude and lag-latitude diagrams show either the equatorially (latitude) averaged $\left(10^{\circ} \mathrm{S}-10^{\circ} \mathrm{N}\right)$ or zonally (longitude) averaged $\left(80^{\circ} \mathrm{E}-\right.$ $\left.100^{\circ} \mathrm{E}\right)$ intraseasonal precipitation anomalies and $\mathrm{u} 850$ anomalies correlated against intraseasonal precipitation at an Indian Ocean reference point $\left(75^{\circ} \mathrm{E}-100^{\circ} \mathrm{E}, 10^{\circ} \mathrm{S}-5^{\circ} \mathrm{N}\right)$. Similar figures can also be produced for other key variables and regions following the definitions of Waliser et al. (2009). To further explore the MJO intraseasonal variability, the wavenumber-frequency spectra for each season is calculated for individual variables. In addition, we also produce cross-spectral plots to quantify the coherence and phase relationships between precipitation and $\mathrm{u} 850$.

\section{Available Namelists and Diagnostics}

Namelists are stored in $\mathrm{nml} /$

- namelist_mjo_mean_state.xml

- namelist_mjo_daily.xml

Diagnostics are stored in diag_scripts/

- mjo_univariate_eof.ncl

- mjo_wave_freq.ncl

- mjo_precip_u850-200_propagation.ncl

- mjo_precip_uwnd_variance.ncl

- mjo_olr_u850-200_cross_spectra.ncl

- mjo_olr_u850_200_ceof.ncl

- mjo_olr_uv850_ceof_life_cycle.ncl

- mjo_precip_u850_basic_month.ncl 


\section{User settings}

User setting files (cfg files) are stored in $\mathrm{nml} / \mathrm{cfg} \_\mathrm{mjo} /$

\section{Variables}

- $\operatorname{pr}$ (atmos, daily/monthly mean, longitude latitude time)

- rlut (atmos, daily mean, longitude latitude time)

- ua (atmos, daily/monthly mean, longitude latitude level time)

- va (atmos, daily mean, longitude latitude level time)

\section{Observations and Reformat Scripts}

Note: (1) obs4mips data can be used directly without any preprocessing; (2) see headers of reformat scripts for non-obs4mips data for download instructions.

- ERA-Interim (ua, monthly means)

Reformat script: reformat_scripts/obs/reformat_obs_ERA-Interim.ncl

- GPCP-1DD (pr, daily means - obs4mips)

- $\quad$ GPCP-SG (pr, monthly means - obs4mips)

- NCEP (ua, va, daily means)

Reformat script: reformat_scripts/obs/reformat_obs_NCEP_daily.ncl

- NOAA-PSD-Interp (rlut, daily means)

Reformat script: reformat_scripts/obs/reformat_obs_NOAA-PSD-Interp-rlut.ncl

\section{References}

Kanamitsu, M., Kousky, V., van den Dool, H., Jenne, R., and Fiorino, M.: The NCEP-NCAR 50-Year Reanalysis: Monthly Means CD-ROM and Documentation, B. Am. Meteorol. Soc., 82, 247-267, 2001.

Kim, D., Sperber, K., Stern, W., Waliser, D., Kang, I. S., Maloney, E., Wang, W., Weickmann, K., Benedict, J., Khairoutdinov, M., Lee, M. I., Neale, R., Suarez, M., Thayer-Calder, K., and Zhang, G.: Application of MJO Simulation Diagnostics to Climate Models, J. Climate, 22, 6413-6436, 2009.

Liebmann, B. and Smith, C. A.: Description of a complete (interpolated) outgoing longwave radiation data set, B. Am. Meteorol. Soc., 77, 1275-1277, 1996.

Waliser, D., Sperber, K., Hendon, H., Kim, D., Wheeler, M., Weickmann, K., Zhang, C., Donner, L., Gottschalck, J., Higgins, W., Kang, I. S., Legler, D., Moncrieff, M., Vitart, F., Wang, B., Wang, W., 
Woolnough, S., Maloney, E., Schubert, S., Stern, W., and Oscillation, C. M.-J.: MJO Simulation Diagnostics, J. Climate, 22, 3006-3030, 2009. 


\section{Example Plots}
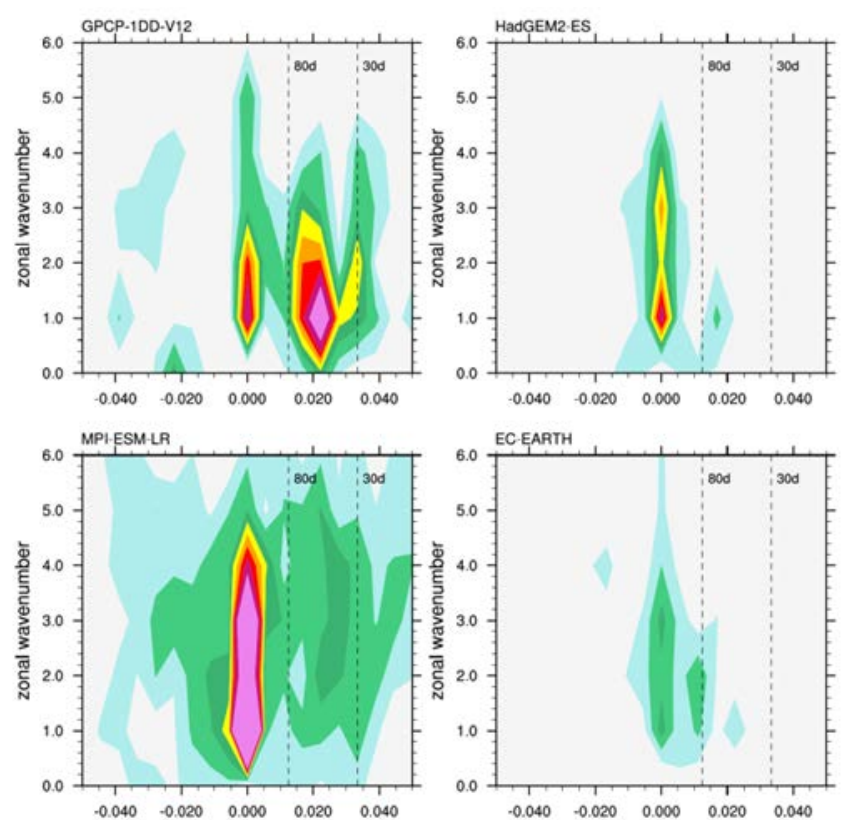

$\begin{array}{llllllll}0.01 & 0.015 & 0.02 & 0.025 & 0.03 & 0.035 & 0.04 & 0.05\end{array}$

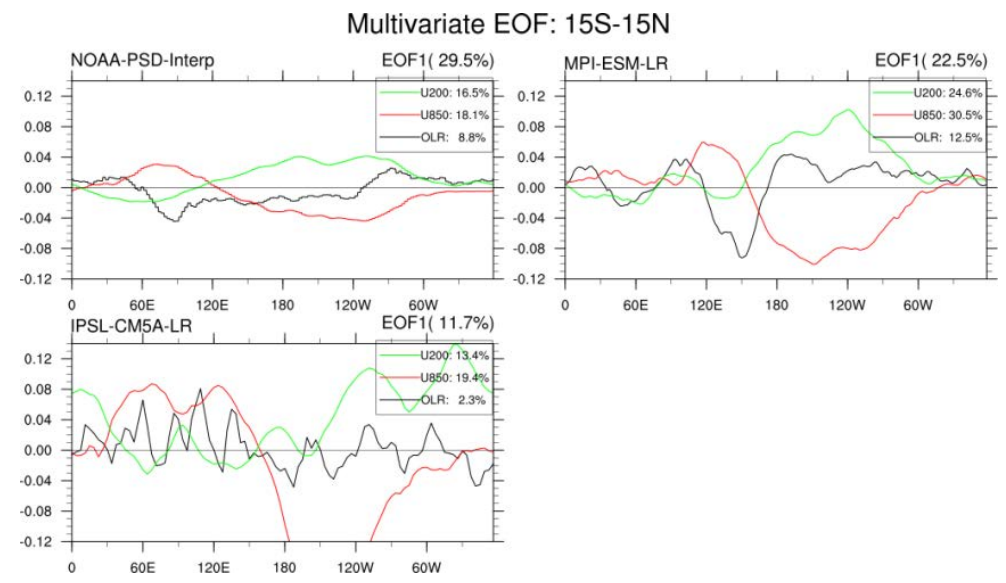




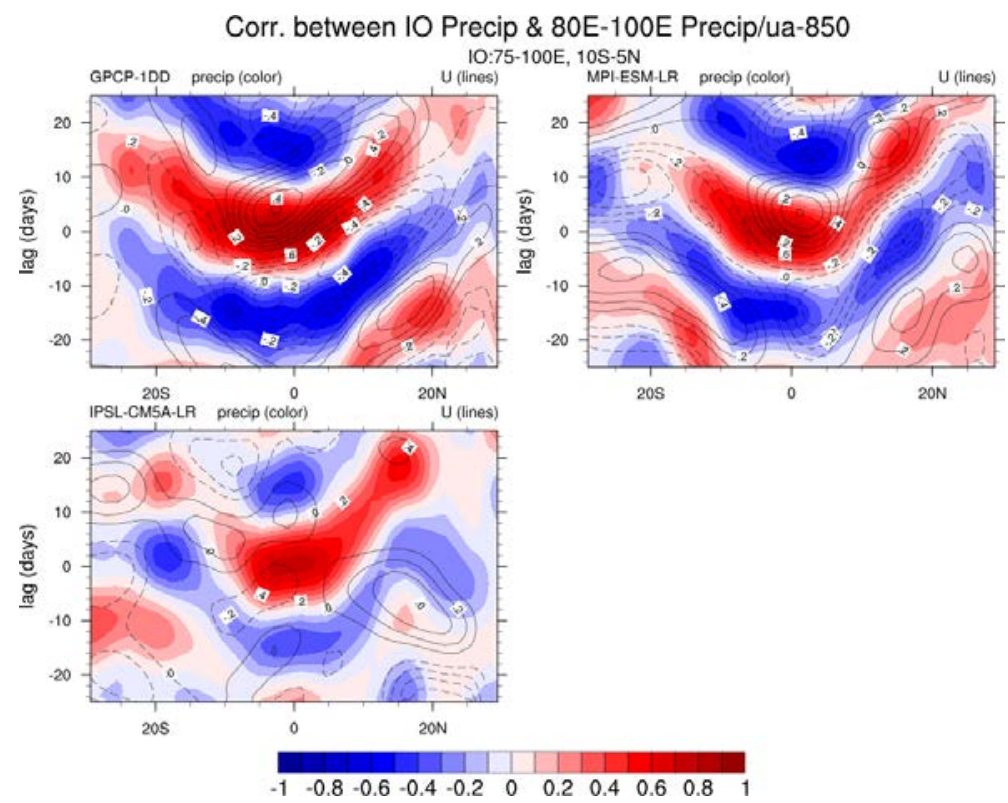

summer
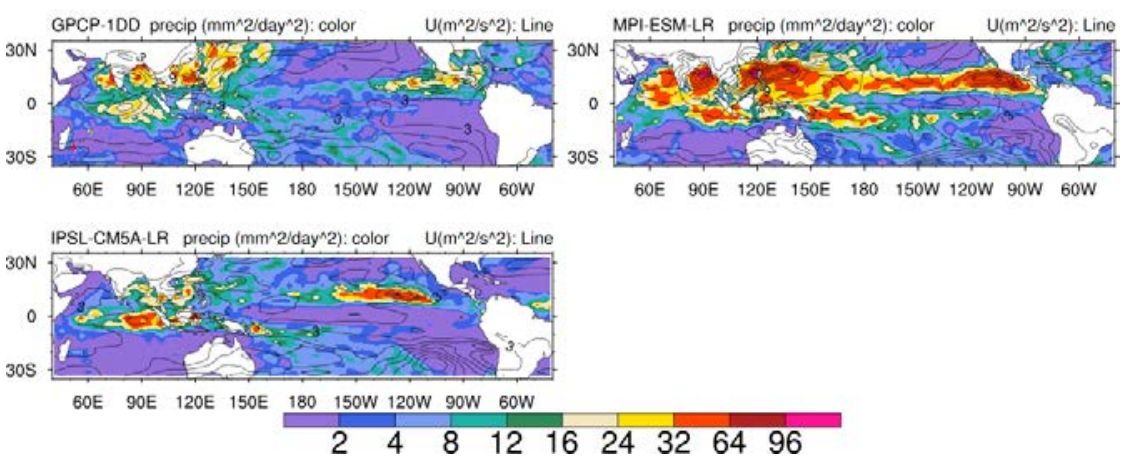


\section{NCAR's Climate Variability Diagnostics Package (CVDP)}

\section{Overview}

The Climate Variability Diagnostics Package (CVDP) developed by NCAR's Climate Analysis Section (Phillips et al., 2014) has been implemented into the ESMValTool in order to be able to run it within this framework and alongside the ESGF on CMIP output. CVDP can be used to evaluate the major modes of climate variability including ENSO, PDO, AMO, Northern and Southern Annular Modes (NAM and SAM), North Atlantic Oscillation (NAO), Pacific North and South American teleconnection patterns (PNA and PSA). In addition it calculates global trend maps and index time series for the above modes and the North Pacific Index, the Tropical North Atlantic SST, Tropical South Atlantic SST, Tropical Indian Ocean SST, Niño1+2, Niño3, and Niño4 times series, and the Indian Ocean Dipole (IOD).

CVDP is developed as a standalone tool outside the ESMValTool. Once a new version of CVDP is released, the ESMValTool will be updated accordingly. Therefore, the structure of CVDP was kept as is and a wrapper has been written to be able to run CVDP directly within the ESMValTool.

\section{Available Namelists and Diagnostics}

Namelists are stored in $\mathrm{nml} /$

- namelist_CVDP.xml

Diagnostics are stored in diag_scripts/

Wrapper scripts to run CVDP within the framework of the ESMValTool

- cvdp_obs.ncl: run for each variable separately with observational data available; renames the ESMValTool output (observations) following the filename conventions of the CVDP and creates the CVDP namelist "namelist_obs".

- cvdp_ocean.ncl: renames the ESMValTool output (ocean variables) following the filename conventions of the CVDP.

- cvdp_atmos.ncl: renames the ESMValTool output (atmosphere variables) following the filename conventions of the CVDP and creates the CVDP namelist "namelist" containing the models. The script then runs the CVDP via a call to the wrapper script cvdp_driver.ncl.

\section{User settings}

User setting files (cfg files) are stored in $\mathrm{nml} / \mathrm{cfg} \_\mathrm{CVDP} /$

(1) crdp_obs.ncl

Required diag_script_info attributes

- obs_ref: list of reference data sets (observations) (array) 
(2) cvdp_driver.ncl (called by cvdp_atmos.ncl)

The wrapper script cvdp_driver.ncl sets the user options for the CVDP.

\section{Variables}

- ts (atmos, monthly mean, longitude latitude time)

- psl (atmos, monthly mean, longitude latitude time)

- $\quad \operatorname{tas}$ (atmos, monthly mean, longitude latitude time)

- $\operatorname{pr}$ (atmos, monthly mean, longitude latitude time)

- msftmyz (ocean, monthly mean, latitude level basin time)

\section{Observations and Reformat Scripts}

Note: (1) obs4mips data can be used directly without any preprocessing; (2) see headers of reformat scripts for non-obs4mips data for download instructions.

- $\quad$ Sea Surface Temperature (SST): HadISST (1870 - 2005)

Reformat script: reformat_scripts/obs/reformat_obs_HadISST.ncl

- Sea Level Pressure (PSL): ERA-Interim/IFS-Cy31r2 (1979 - 2013)

Reformat script: reformat_scripts/obs/reformat_obs_ERA-Interim.ncl

- 2m Air Temperature (TAS): NCEP (1979 - 2005)

Reformat script: reformat_scripts/obs/reformat_obs_NCEP.ncl

- Precipitation (PR): GPCP-SG (1979 - 2005) (obs4mips)

\section{References}

Danabasoglu, G., S. G. Yeager, Y. -O. Kwon, J. J. Tribbia, A. S. Phillips, and J. W. Hurrell, 2012. Variability of the Atlantic Meridional Overturning Circulation in CCSM4. J. Climate, 25, 51535172, doi: 10.1175/JCLI-D-11-00463.1 (AMOC).

Deser, C., M. A. Alexander, S. -P. Xie, and A. S. Phillips, 2010: Sea surface temperature variability: patterns and mechanisms. Ann. Rev. Mar. Sci., 2010.2, 115-143, doi: 10.1146/annurev-marine-120408151453 (PDO).

Deser, C., A. S. Phillips, R. A. Tomas, Y. Okumura, M. A. Alexander, A. Capotondi, J. D. Scott, Y. -O. Kwon, and M. Ohba, 2012: ENSO and Pacific Decadal Variability in Community Climate System Model Version 4. J. Climate, 25, 2622-2651, doi: 10.1175/JCLI-D-11-00301.1 (ENSO Spatial Composites).

Hurrell, J. W., and C. Deser, 2009: North Atlantic climate variability: The role of the North Atlantic Oscillation. J. Mar. Syst., 78, 28-41, doi: 10.1016/j.jmarsys.2008.11.026 (NAO). 
Mantua, N. J., S. R. Hare, Y. Zhang, J. M. Wallace, and R. Francis, 1997: A Pacific interdecadal climate oscillation with impacts on salmon production. Bull. Amer. Met. Soc., 1069-1079 (PDO).

Phillips, A. S., Deser, C., and Fasullo, J.: Evaluating Modes of Variability in Climate Models, Eos Trans. AGU, 95(49), 453-455, 2014 (Overview Paper of Climate Variability Diagnostics Package).

Thompson, D. W. J. and J.M. Wallace, 2000: Annular modes in the extratropical circulation. Part I: Monthto-month variability. J. Climate, 13, 1000-1016 (NAM).

Trenberth, K. E., and D. J. Shea, 2006: Atlantic hurricanes and natural variability in 2005, Geophys. Res. Lett., 33, L12704, doi: 10.1029/2006GL026894 (AMO). 


\section{Example Plots}

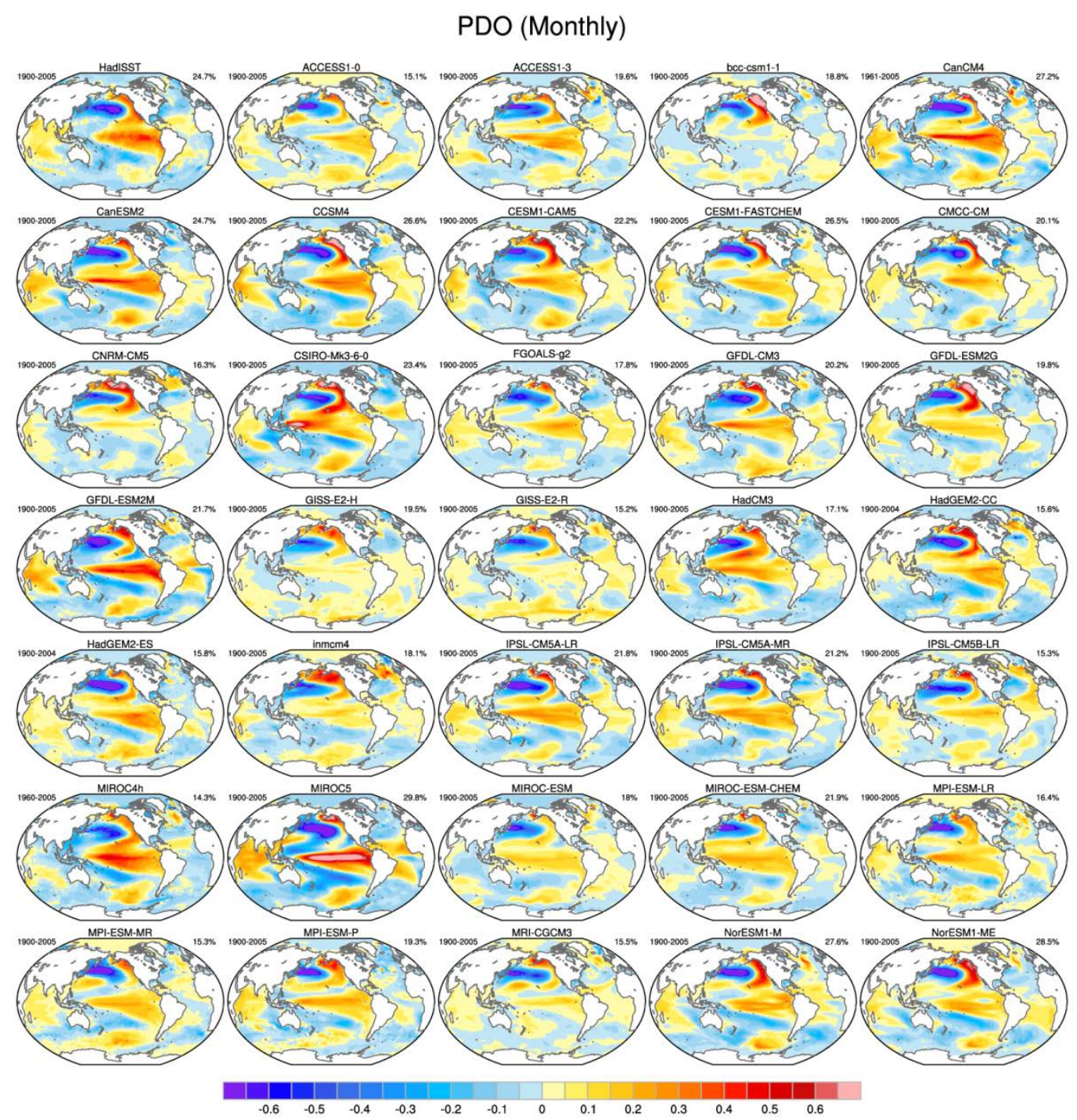

Figure 21 The leading empirical orthogonal function (EOF) of monthly sea surface temperature (SST) anomalies over the North Pacific (after removing the global mean SST anomaly) based on the HadISST observational data set (top left) during 1900-2005, and (remaining panels) several model simulations of the Coupled Model Intercomparison Project Phase 5 (CMIP5), for the models MIROC4h and CanCM4 during 1960-2005. 

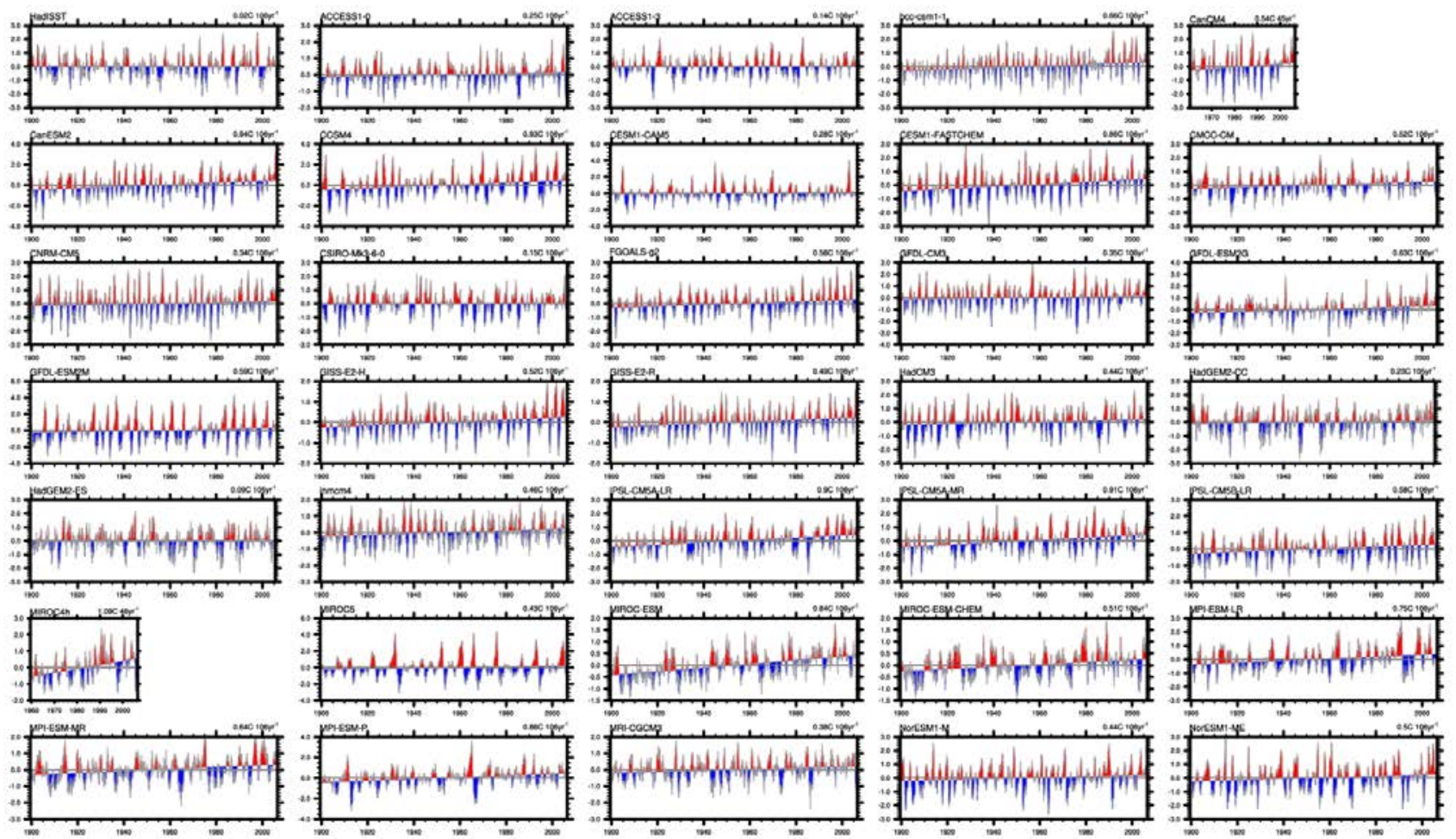

Figure 22 The weighted area average of monthly SST anomalies in the region $5^{\circ} \mathrm{S}-5^{\circ} \mathrm{N}, 190^{\circ}-240^{\circ} \mathrm{E}$ also known as the "Niño-3.4 Index" (Trenberth et al., 2002) based on the HadISST observational data set (top left) for the period 19002005, and (remaining panels) several model simulations of the Coupled Model Intercomparison Project Phase 5 (CMIP5), for the models MIROC4h and CanCM4 during 1960-2005. The red/blue shading on the Niño3.4 time series denotes positive/negative deviations from the best-fit linear trend line. 


\section{La Niña Composite $\left(3^{\circ} \mathrm{S}: 3^{\circ} \mathrm{N}\right)$}
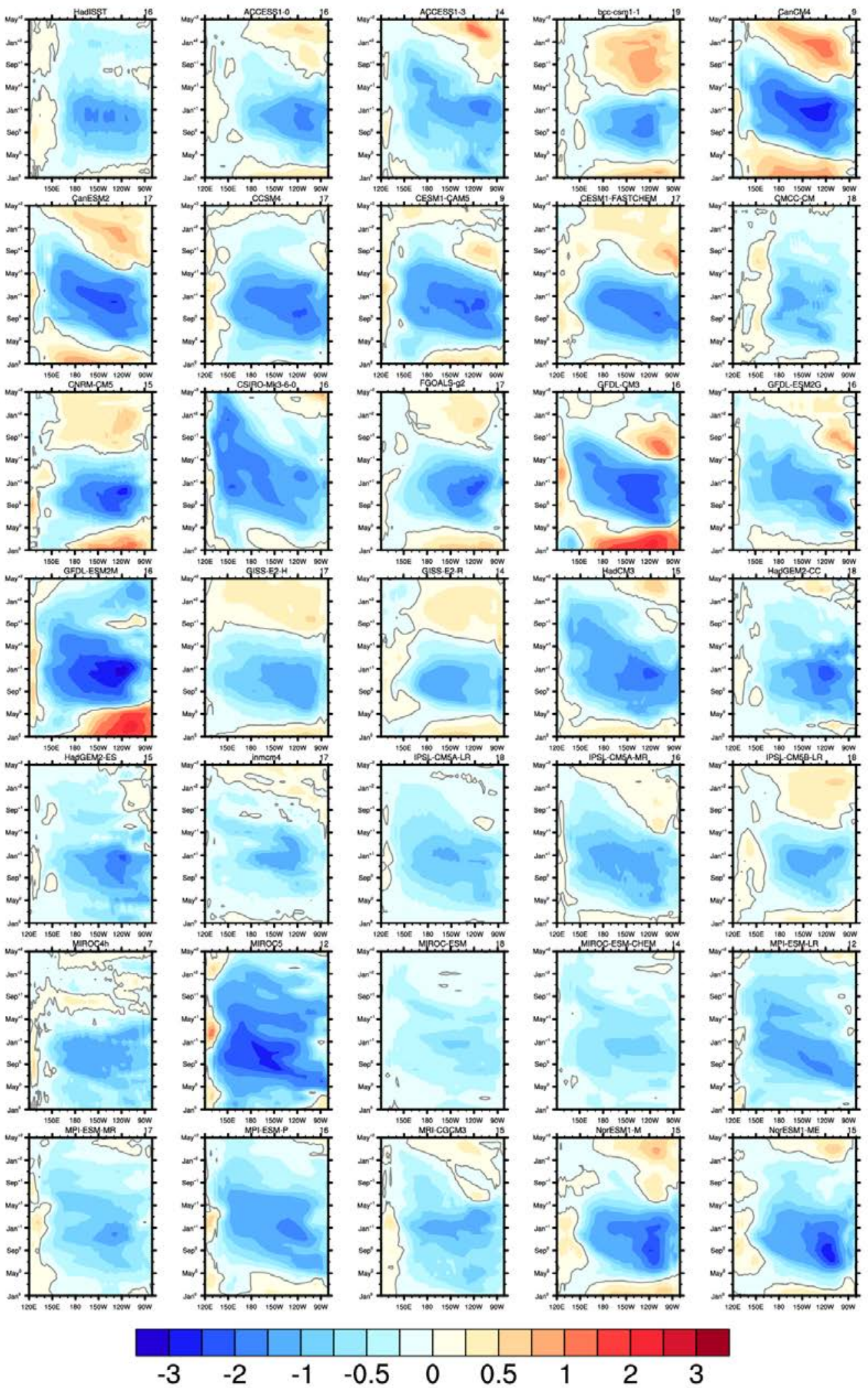

Figure 23 Longitude-latitude sections of composite SST anomalies along the equator $\left(3^{\circ} \mathrm{N}-3^{\circ} \mathrm{S}\right)$ for La Niña based on the HadISST oberservational data set (top left) for the period 1900-2005, and (remaining panels) several model simulations of the Coupled Model Intercomparison Project Phase 5 (CMIP5), for the models MIROC4h and CanCM4 during 1960-2005. The number at the top right of each panel indicates the number of events used for the composite. 


\section{AMO (Monthly)}
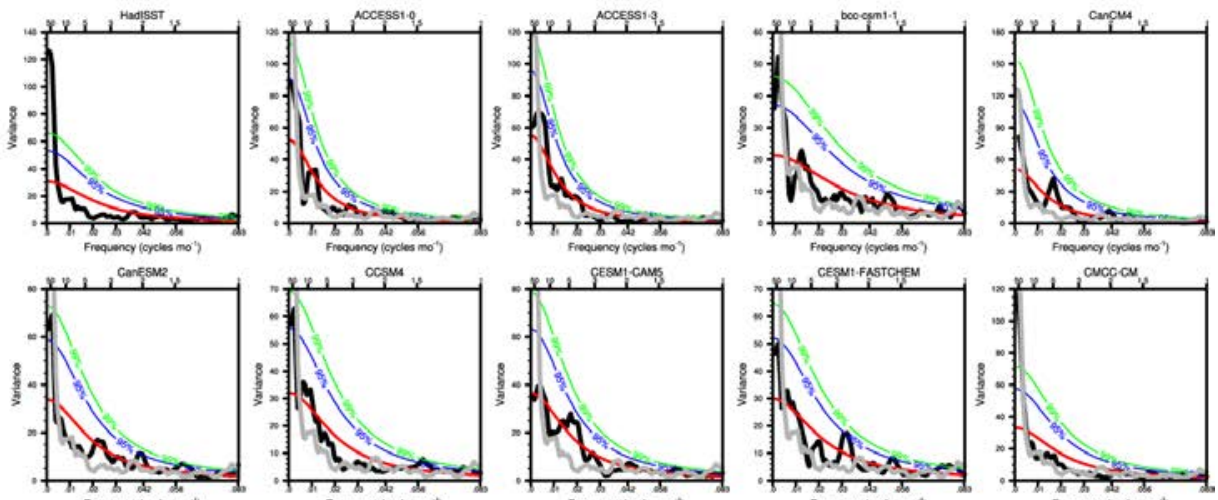

CESUI:FAST
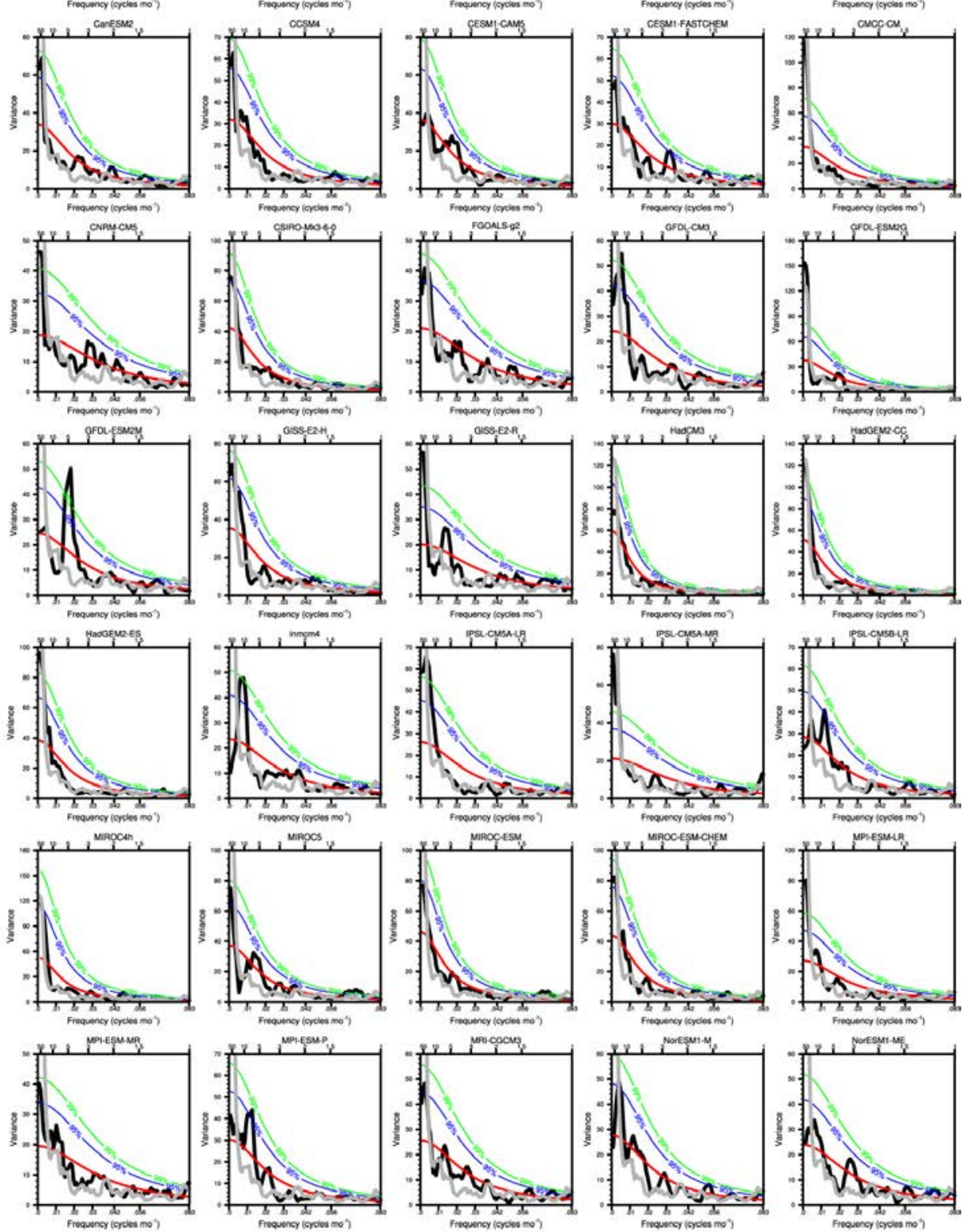

Figure 24 The power spectrum as a function of frequency in cycle per month of the Niño-3.4 SST index for the HadISST observational data set (top left) for the period 1900-2005, and (remaining panels) several model simulations of the Coupled Model Intercomparison Project Phase 5 (CMIP5), for the models MIROC4h and CanCM4 during 19602005. The black line denotes the spectrum. The red line indicates the best-fit first-order Markov red noise spectrum, the blue line its $95 \%$ and the green line its $99 \%$ confidence bounds. The observational spectrum is overlaid in gray on each model spectrum if available. 


\section{Ozone and associated climate impacts}

\section{Overview}

This namelist implemented into the ESMValTool to evaluate atmospheric chemistry and the climate impact of stratospheric ozone changes reproduces selected plots from Eyring et al. (2013), i.e. their figs. 1, 2, 4, 6, 7 , 10, and 11. These include calculation of the zonally averaged seasonal cycle of total ozone columns ("fig. 1"), time series of the total ozone averaged over given regions ("fig. 2"), climatological mean tropospheric ozone columns ("fig. 4"), stratospheric ozone time series ("fig. 6"), differences in vertical ozone profiles between the 2090s and 2000s ("fig. 7"), trends in annual mean ozone, temperature, and jet position ("fig. 10 "), and trend relationships between ozone and temperature and jet position ("fig. 11").

\section{Available Namelists and Diagnostics}

Namelists are stored in $\mathrm{nml} /$

- namelist_eyring13jgr.xml

Diagnostics are stored in diag_scripts/

- eyring13jgr_fig01.ncl: calculates seasonal cycles of zonally averaged total ozone columns.

- eyring13jgr_fig02.ncl: time series of area-weighted total ozone from 1960-2005 for the annual mean averaged over the global domain $\left(90^{\circ} \mathrm{S}-90^{\circ} \mathrm{N}\right)$, Tropics $\left(25^{\circ} \mathrm{S}-25^{\circ} \mathrm{N}\right)$, northern mid-latitudes $\left(35^{\circ} \mathrm{N}\right.$ $\left.60^{\circ} \mathrm{N}\right)$, southern mid-latitudes $\left(35^{\circ} \mathrm{S}-60^{\circ} \mathrm{S}\right)$, and the March and October mean averaged over the Arctic $\left(60^{\circ} \mathrm{N}-90^{\circ} \mathrm{N}\right)$ and the Antarctic $\left(60^{\circ} \mathrm{S}-90^{\circ} \mathrm{S}\right)$.

- eyring13jgr_fig04.ncl: climatological annual mean tropospheric ozone columns (geographical distribution).

- eyring13jgr_fig06.ncl: 1980 baseline-adjusted stratospheric column ozone time series from 19602100 .

- eyring13jgr_fig07.ncl: differences in vertically resolved ozone between the 2090s and 2000s for the annual mean averaged over the global domain $\left(90^{\circ} \mathrm{S}-90^{\circ} \mathrm{N}\right)$, Tropics $\left(25^{\circ} \mathrm{S}-25^{\circ} \mathrm{N}\right)$, northern midlatitudes $\left(35^{\circ} \mathrm{N}-60^{\circ} \mathrm{N}\right)$, southern mid-latitudes $\left(35^{\circ} \mathrm{S}-60^{\circ} \mathrm{S}\right)$, and the March and October mean averaged over the Arctic $\left(60^{\circ} \mathrm{N}-90^{\circ} \mathrm{N}\right)$ and the Antarctic $\left(60^{\circ} \mathrm{S}-90^{\circ} \mathrm{S}\right)$.

- eyring13jgr_fig10.ncl: trends in annual mean near-global $\left(82.5^{\circ} \mathrm{S}-82.5^{\circ} \mathrm{N}\right)$ ozone at $50 \mathrm{hPa}$ and temperature at $100 \mathrm{hPa}$, September-October-November-December (SOND) ozone at $50 \mathrm{hPa}$ over Antarctica $\left(60^{\circ} \mathrm{S}-90^{\circ} \mathrm{S}\right)$, October-November-December-January (ONDJ) temperature at $100 \mathrm{hPa}$ over Antarctica $\left(60^{\circ} \mathrm{S}-90^{\circ} \mathrm{S}\right)$, DJF SH jet position at $850 \mathrm{hPa}$, and DJF upper tropospheric tropical $\left(30^{\circ} \mathrm{S}-\right.$ $30^{\circ} \mathrm{N}$ ) temperatures at $250 \mathrm{hPa}$. The trends are calculated over 1979-2005 for the past and over 20062050 for the future.

- eyring13jgr_fig11.ncl: trend relationship between annual mean near-global $\left(82.5^{\circ} \mathrm{S}-82.5^{\circ} \mathrm{N}\right)$ ozone at $50 \mathrm{hPa}$ and temperature at $100 \mathrm{hPa}$, SOND ozone at $50 \mathrm{hPa}$ and ONDJ temperature at $100 \mathrm{hPa}$ over Antarctica $\left(60^{\circ} \mathrm{S}-90^{\circ} \mathrm{S}\right)$, SOND ozone at $50 \mathrm{hPa}$ and DJF jet position at $850 \mathrm{hPa}$; and DJF $250 \mathrm{hPa}$ tropical $\left(30^{\circ} \mathrm{S}-30^{\circ} \mathrm{N}\right)$ temperatures and DJF jet position at $850 \mathrm{hPa}$. The trends are calculated over 1979-2005 for the past and over 2006-2050 for the future. 


\section{User settings}

User setting files (cfg files) are stored in $\mathrm{nml} / \mathrm{cfg}$ _eyring13jgr

(1) eyring13jgr_fig01.ncl

diag_script_info attributes

- rgb_file: path + filename of color table (e.g., "diag_scripts/lib/ncl/rgb/eyring_toz.rgb")

- $\quad$ styleset: style set ("DEFAULT, "CMIP5")

- font: overrides default font $\quad$ (e.g., $21 ;$ see www.ncl.ucar.edu/Document/Graphics/Resources/tx.shtml\#txFont)

- range_option: $0=$ as in $\mathrm{nml}, 1=$ overlapping time period

- lbLabelBarOn: plot a label bar (True, False)

- $\quad$ e13fig0 $01_{-}=$"True"

- e13fig01_list_chem_mod: list of models in the group "chem" (array of strings, default = $(/ " \mathrm{All} " /))$

- e13fig01_list_chem_mod_string: plotting label for group "chem", e.g., "CMIP5"

- e13fig01_list_nochem_mod: list of models in the group "nochem" (array of strings, default = $(/ " " /))$

- e13fig01_list_nochem_mod_string: plotting label for group “nochem", e.g., "NoChem"

- e13fig01_diff_ref: name of reference model for difference plots, e.g., "NIWA"

(2) eyring13jgr_fig02.ncl

diag_script_info attributes

- e13fig02_latrange: min. and max. latitude of the regions (n-element array of 2-element pairs, e.g., $(/(/-90,90 /),(/-90,-60 /) /))$; one pair of latitudes is required for each season (see below)

- $\quad$ styleset: style set ("DEFAULT, "CMIP5")

- e13fig02_season: seasons (n-element array of strings, "ANN", "JAN", "FEB", "MAR", "DJF", "SON", etc.)

- e13fig02_XMin: min. X-values (start years) for plotting (n-element array, e.g., (/1960., 1960./)); array is required to have the same number of elements as "seasons" (see above)

- e13fig02_XMax: max. x-values (end years) for plotting (n-element array, e.g., (/2005., 2005./)); array is required to have the same number of elements as "seasons" (see above)

- e13fig02_YMin: min. y-values for plotting (n-element array, e.g., (/260., 150./)); array is required to have the same number of elements as "seasons" (see above) 
- e13fig02_YMax: max. y-values for plotting (n-element array, e.g., (/340.,500./)); array is required to have the same number of elements as "seasons" (see above)

- e13fig02_legend: plot legend (string, e.g., "True")

- e13fig02_legend_MMM: include multi model mean in legend (string, e.g., "False")

- list_chem_mod: list of models in the group "chem" (array of strings, default = (/"All"))

- list_nochem_mod: list of models in the group "nochem" (array of strings, default = $(/$ "None"/))

(3) eyring13jgr_fig04.ncl

diag_script_info attributes

- styleset: style set (“DEFAULT, "CMIP5")

- font: overrides default font (e.g., $21 ;$ see www.ncl.ucar.edu/Document/Graphics/Resources/tx.shtml\#txFont)

- range_option: $0=$ as in $\mathrm{nml}, 1=$ overlapping time period

- lbLabelBarOn: plot a label bar (True, False)

- $\quad$ e13fig04_= "True"

- e13fig04_list_chem_mod: list of models in the group "chem" (array of strings, default = $(/ " \mathrm{All} " /))$

- e13fig04 list chem mod string: plotting label for group "chem", e.g., "CMIP5"

- e13fig04_list_nochem_mod: list of models in the group "nochem" (array of strings, default = $(/ " " /))$

- e13fig04_list_nochem_mod_string: plotting label for group "nochem”, e.g., "NoChem"

- e13fig04_diff_ref: name of reference model for difference plots, e.g., "AURA-MLS-OMI"

- mpProjection: map projection, optional (e.g., "CylindricalEquidistant") (see http://www.ncl.ucar.edu/Document/Graphics/Resources/mp.shtml\#mpProjection for available projections)

(4) eyring13jgr_fig06.ncl

diag_script_info attributes

- e13fig06_latrange: min. and max. latitude of the regions (n-element array of 2-element pairs, e.g., $(/(/-90,90 /),(/-90,-60 /) /))$; one pair of latitudes is required for each season (see below)

- styleset: style set (“DEFAULT”, "CMIP5")

- e13fig06_season: seasons (n-element array of strings, "ANN", "JAN", "FEB", "MAR", "DJF", "SON", etc.)

- e13fig06_baseline_adj: do baseline adjustment (string: "True", "False") 
- e13fig06_baseline: year for baseline adjustment (e.g., 1980)

- e13fig06_mod_plot: "MMT" = plot of the MultiModel mean of each scenario and selection "list_chem_mod" and "list_nochem_mod"; "IMT" = plot of each single model trend; "RAW" = plot of each model as raw data

- e13fig06_mod_plot_CI: plot confidence interval (string: "True", "False"); for e13fig06_mod_plot = "MMT” only!

- e13fig06_mod_plot_PI: plot prediction interval (string: "True", "False"); for e13fig06_mod_plot $=$ "MMT" only!

- e13fig06_XMin: min. X-values (start years) for plotting (n-element array, e.g., (/1960., 1960./)); array is required to have the same number of elements as "seasons" (see above)

- 13fig06_XMax: max. x-values (end years) for plotting (n-element array, e.g., (/2010., 2010./)); array is required to have the same number of elements as "seasons" (see above)

- e13fig06_YMin: min. y-values for plotting (n-element array, e.g., (/260., 150./)); array is required to have the same number of elements as "seasons" (see above)

- e13fig06_YMax: max. y-values for plotting (n-element array, e.g., (/330., 500./)); array is required to have the same number of elements as "seasons" (see above)

- list_chem_mod: list of models in the group "chem" (array of strings, default $=(/$ "All") $)$

- list_nochem_mod: list of models in the group "nochem" (array of strings, default = $(/$ "None"/))

- e13fig06_labels_exp_esp: specify experiment name (string: "True", "False"); only if e13fig06_mod_plot= "IMT" or "RAW"!

(5) eyring13jgr_fig07.ncl

diag_script_info attributes

- e13fig06_latrange: min. and max. latitude of the regions (n-element array of 2-element pairs, e.g., $(/(/-90,90 /),(/-90,-60 /) /))$; one pair of latitudes is required for each season (see below)

- $\quad$ styleset: style set ("DEFAULT", "CMIP5")

• e13fig07_season: seasons (n-element array of strings, "ANN", "JAN", "FEB", "MAR", "DJF", "SON", etc.)

- e13fig07_period1: start and end year of "period1" (=2000s), e.g., (/2000., 2009/)

- e13fig07_period2: start and end year of "period2" (=2090s), e.g., (/2090., 2099/)

- e13fig07_XMin: min. $x$-values for plotting (n-element array, e.g., (/-2., $-2 . /))$; array is required to have the same number of elements as "seasons" (see above)

- 13fig07_XMax: max. x-values for plotting (n-element array, e.g., (/2., 12./)); array is required to have the same number of elements as "seasons" (see above)

- list_chem_mod: list of models in the group "chem" (array of strings, default = (/"All")) 
- list_nochem_mod: list of models in the group "nochem" (array of strings, default = $(/$ "None" $/))$

(6) eyring13jgr_fig10.ncl

diag_script_info attributes

- e13fig10_latrange: min. and max. latitude of the regions (n-element array of 2-element pairs, e.g., $(/(/-30,30 /) /))$; one pair of latitudes is required for each season (see below)

- $\quad$ styleset: style set ("DEFAULT", "CMIP5")

- e13fig10_season: seasons (n-element array of strings, e.g., "ANN", "JAN", "FEB", "MAR", "DJF", "SON", etc.)

- e13fig10_lev: vertical level (in hPa)

- plot_number: string used for plot labeling / sub-figure (e.g., “(a)”)

- list_chem_mod: list of models in the group "chem" (array of strings, default $=(/$ "All"))

- list_nochem_mod: list of models in the group "nochem" (array of strings, default = $(/$ "None" $/))$

(7) eyring13jgr_fig11.ncl

diag_script_info attributes

- $\quad$ styleset: style set ("DEFAULT", "CMIP5")

- e13fig11_V0_units: unit label for "variable 0" (x-axis) (string)

- e13fig11_V1_units: unit label for "variable 1" (y-axis) (string)

- e13fig11_V0_latrange: min. and max. latitude of the region for "variable 0"

- e13fig11_V1_latrange: min. and max. latitude of the region for "variable 1"

- e13fig11_V0_season: season for "variable 0" (e.g., "yearly")

- e13fig11_V1_season: season for "variable 1" (e.g., "yearly")

- e13fig10_V0_lev: vertical level (in hPa) for "variable 0"

- e13fig10_V1_lev: vertical level (in hPa) for "variable 1"

- plot_number: string used for plot labeling / sub-figure (e.g., "(a)”)

- e13fig11_XMin: min. $x$-value for plotting

- e13fig11_XMax: max. x-value for plotting

- e13fig11_YMin: min. y-value for plotting

- e13fig11_YMax: max. y-value for plotting

- list_chem_mod: list of models in the group "chem" (array of strings, default = empty) 
- list_nochem_mod: list of models in the group "nochem" (array of strings, default = empty)

\section{Variables}

- $\quad \operatorname{tro3}$ (atmos, monthly mean, longitude latitude lev time)

- ta (atmos, monthly mean, longitude latitude lev time)

- ua (atmos, monthly mean, longitude latitude lev time)

\section{Observations and Reformat Scripts}

- Total column ozone (toz): NIWA (Bodeker et al., 2005)

Reformat scripts:

o reformat_scripts/obs/reformat_obs_NIWA.ncl

- Tropospheric column ozone (tropoz): MLS/OMI (Ziemke et al., 2006)

Reformat script: reformat_scripts/obs/reformat_obs_AURA-MLS-OMI.ncl

\section{References}

Eyring, V., J. M. Arblaster, I. Cionni, J. Sedlacek, J. Perlwitz, P. J. Young, S. Bekki, D. Bergmann, P. Cameron-Smith, W. J. Collins, G. Faluvegi, K.-D. Gottschaldt, L. W. Horowitz, D. E. Kinnison, J.-F. Lamarque, D. R. Marsh, D. Saint-Martin, D. T. Shindell, K. Sudo, S. Szopa, and S. Watanabe, Longterm ozone changes and associated climate impacts in CMIP5 simulations, J. Geophys. Res. Atmos., 118, doi: 10.1002/jgrd.50316, 2013.

\section{Example Plots}

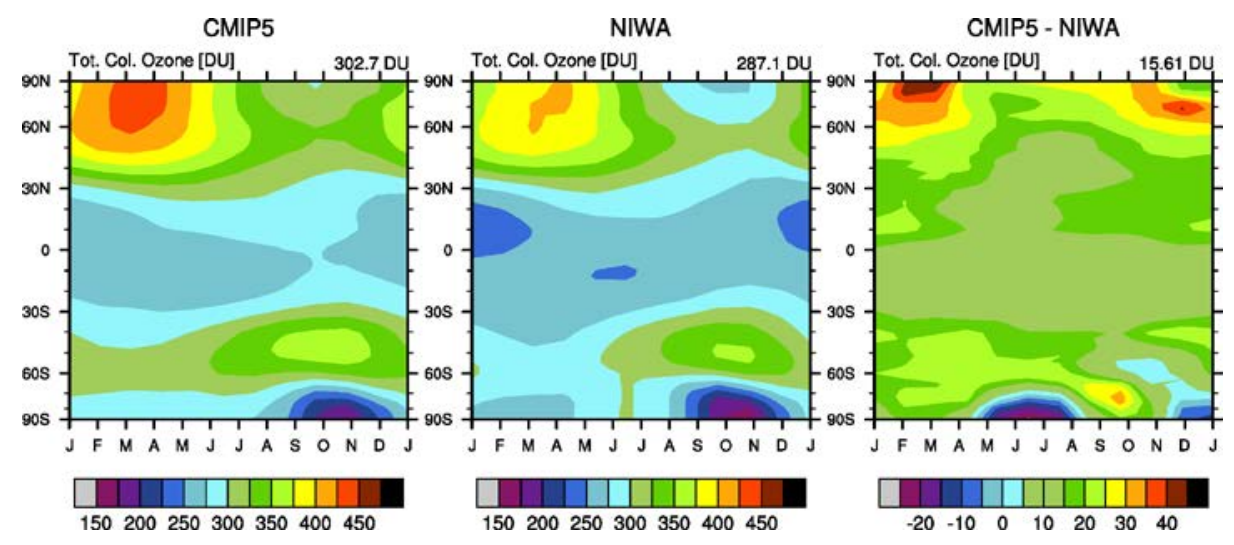

Figure 25 Produced with “eyring13jgr_fig01.ncl”. 


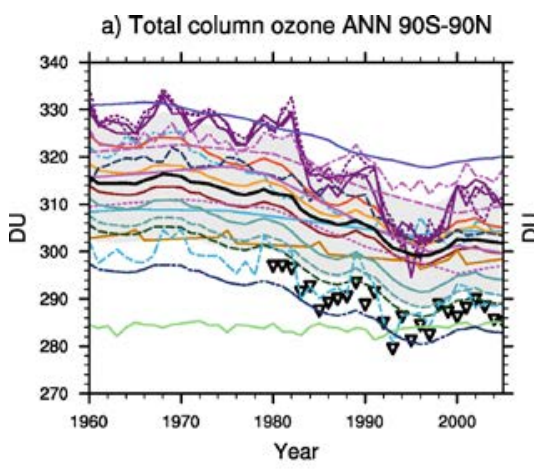

b) Total column ozone OCT 90S-60S

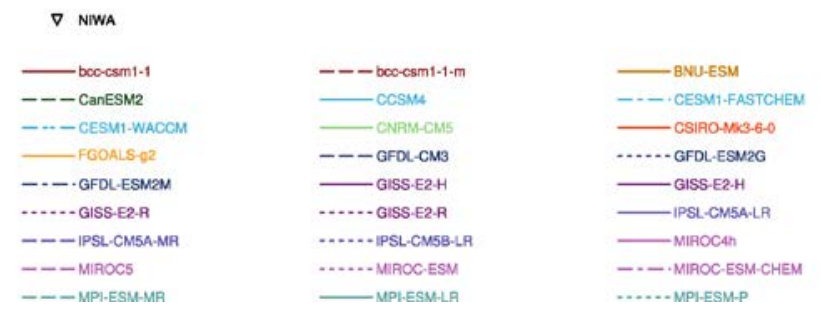

Figure 26 Produced with "eyring13jgr_fig02.ncl".

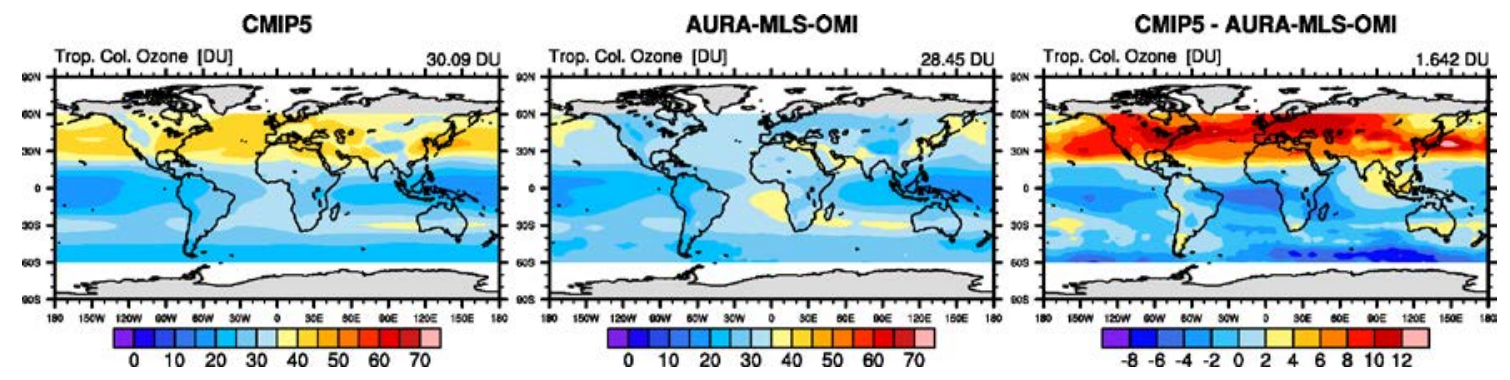

Figure 27 Produced with "eyring13jgr_fig04.ncl".
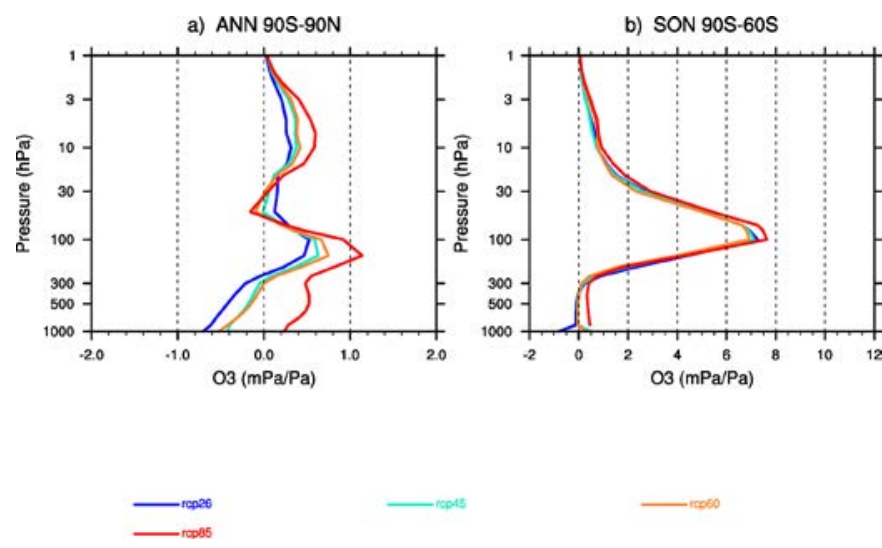

Figure 28 Produced with "eyring13jgr_fig07.ncl". 

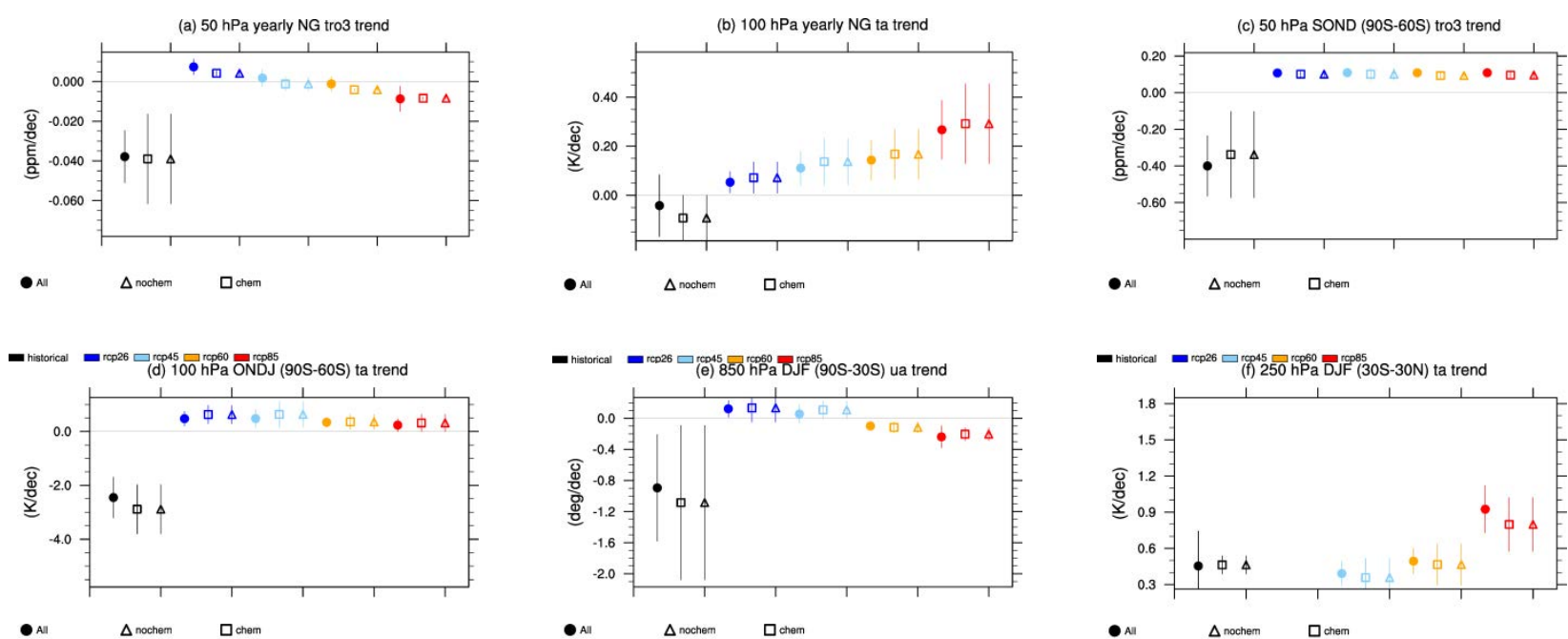

- An $\Delta$ nochem पchom

- Al $\Delta$ nochem Dcher

- MII $\Delta$ nochem Dorem

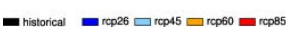

Figure 29 Produced with “eyring13jgr_fig10.ncl”.

(a)

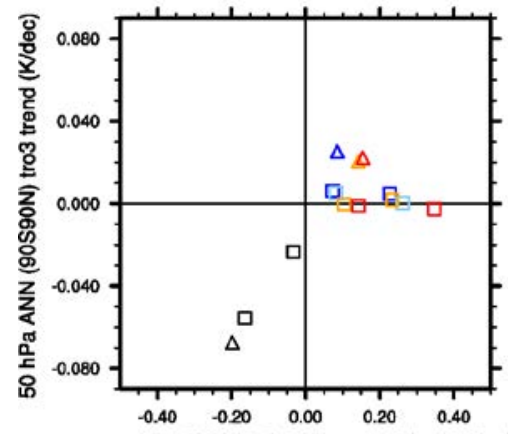

$100 \mathrm{hPa}$ ANN $(90590 \mathrm{~N})$ ta trend (ppmv/dec]

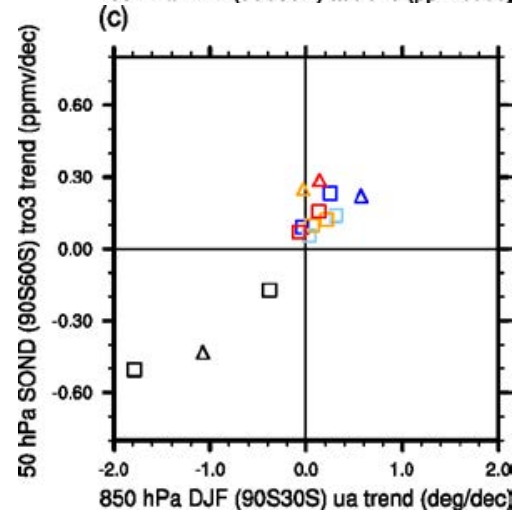

(b)

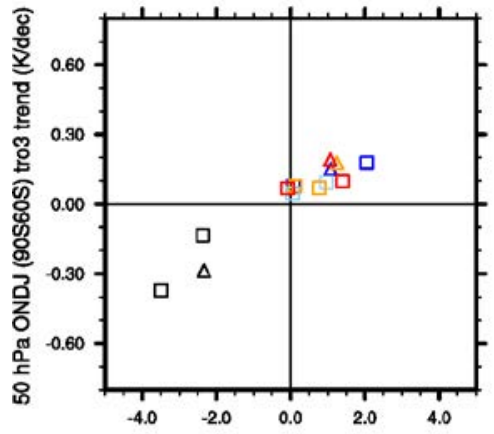

$100 \mathrm{hPa}$ SOND (90560S) ta trend (ppmv/dec)

(d)

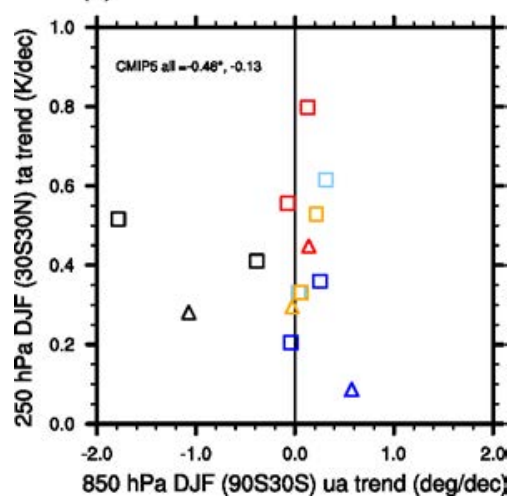

Figure 30 Produced with "eyring13jgr_fig11.ncl”. 


\section{Ozone and some precursors}

\section{Overview}

This namelist provides diagnostics to evaluate the simulated atmospheric composition in ESMs with a focus on ozone and precursor gases such as $\mathrm{NO}_{\mathrm{x}}$. This includes comparisons of simulated tropospheric ozone columns, seasonal cycle and vertical profiles of trace gases with observations. Model data are sampled for the same regions and the same time of year as the observations, monthly data are weighted according to the number of days in each month contributing to the respective campaign climatology.

\section{Available Namelists and Diagnostics}

Namelists are stored in $\mathrm{nml} /$

- namelist_righi15gmd_Emmons.xml

- namelist_righi15gmd_tropo3.xml

Diagnostics are stored in diag_scripts/

- ancyc_lat.ncl: annual cycle contour plots of zonal means.

- Emmons.ncl: profiles of atmospheric trace gas mixing ratios, compared to the in-situ data climatologies of Emmons et al. (2000). Simulation data are sampled for the same regions and the same time of year as the observations. Monthly data are weighted according to the number of days in each month contributing to the respective campaign climatology. Note: simulation data are sampled according to the period specified in the namelist. Observational data may be from different years.

- lat_lon.ncl: plots the geographical distribution of a variable (latitude-longitude plot).

- perfmetrics_grading.ncl: calculates grades according to a given metric with different options for normalization. It requires fields precalculated by perfmetrics_main.ncl (see Performance Metrics for Essential Climate Parameters).

- perfmetrics_grading_collect.ncl: collects results from metrics previously calculated by perfmetrics_grading.ncl and passes them to the plotting functions (see Performance Metrics for Essential Climate Parameters).

- perfmetrics_main.ncl: calculates and (optionally) plots annual/seasonal cycles, zonal means, lat-lon fields and time-lat-lon fields from input monthly 2-d or 3-d ("T2M", "T3Ms") data. The calculated fields can be also plotted as difference w.r.t. a given reference model. They are also used as input to calculate grading metrics (see perfmetrics_grading.ncl) (see Performance Metrics for Essential Climate Parameters).

- perfmetrics_taylor.ncl: calculates grades according to a given metric with different options for normalization. It requires fields precalculated by perfmetrics_main.ncl (see Performance Metrics for Essential Climate Parameters).

- perfmetrics_taylor_collect.ncl: collects results from metrics previously calculated by perfmetrics_taylor.ncl and passes them to the plotting functions (see Performance Metrics for Essential Climate Parameters). 


\section{User settings}

User setting files (cfg files) are stored in $\mathrm{nml} / \mathrm{cfg}$ _righi15gmd/Emmons/ and $\mathrm{nml} / \mathrm{cfg}$ _righi15gmd_tropo3/

(1) ancyc_lat.ncl

Requires diag_script_info attributes

- styleset: style ("DEFAULT, "righi15gmd"; see diag_scripts/lib/ncl/style.ncl for available styles)

- font: font type (see www.ncl.ucar.edu/Document/Graphics/Resources/tx.shtml\#txFont)

Optional diag_script_attributes

- obsfiles

- range_option: $0=$ as in $\mathrm{nml}, 1=$ overlapping time period

- case

- $\mathrm{y} 1$

- $\mathrm{y} 2$

- obsname

- lbLabelBarOn: plot label bar (True/False)

(2) Emmons.ncl

- obsfiles: list of observational files including full pathnames; all files matching the following will be scanned, all suitable data will be used (mandatory), wildcards are allowed.

OBS parameters

The target variable (e.g., "vmro3") + the following extensions give the variable names in the obs file for specific quantities such as mean, standard devition, minimum, etc. Deactivate lines corresponding to parameters that are not in the obs file; either obsvar_mean or obsvar_median is mandatory. "_N" is only used for grading: will use equal weights if disabled. Disabling any other quantity will just cause the corresponding whisker not to be drawn (this may be used to switch off whiskers).

- obsvar_N: extension to assemble variable name for number of observations, e.g., "_N"

- obsvar_mean: extension to assemble variable name for mean, e.g., "_mean"

- obsvar_stddev: extension to assemble variable name for standard deviation, e.g., "_stddev"

- obsvar_min: extension to assemble variable name for minima, e.g, "_min"

- obsvar_max : extension to assemble variable name for maxima, e.g, "_max"

- obsvar_5: extension to assemble variable name for 5\% percentiles, e.g., "_5"

- obsvar_25: extension to assemble variable name for $25 \%$ percentiles, e.g., "_25"

- obsvar_median: extension to assemble variable name for median, e.g., "_median" 
- obsvar_75: extension to assemble variable name for 75\% percentiles, e.g, "_75"

- obsvar_95: extension to assemble variable name for 95\% percentiles, e.g., "_95"

Campaign parameters, expected to be global attributes of each obs file (all mandatory)

- obsatt_campaign: name of the attribute containing the campaign name (plot annotation), e.g., "campaign"

- obsatt_location: name of the attribute containing the region (plot annotation), e.g., "location"

- obsatt_period: name of the attribute containing the time period covered, e.g., "period"

- obsatt_latmin: name of the attribute containing the min. latitude of the region, e.g., "latmin"

- obsatt_latmax: name of the attribute containing the max. latitude of the region, e.g., "latmax"

- obsatt_lonmin: name of the attribute containing the min. longitude of the region, e.g., "lonmin"

- obsatt_lonmax: name of the attribute containing max. longitude of the region, e.g., "lonmax"

Optional FILTER parameters for selection of obs data

- roi: region of interest (4-element array given as (/latmin, latmax, lonmin, lonmax/)) (default $=(/ 90 ., 90 ., 0 ., 360 . /))$

- roi_match: "contained", "center", "overlap", "encloses", "outside" (default: "center"); e.g., "center" = center of observational region (given by global attributes of observational file) must be inside the "region of interest (roi)" for the data to be considered

- poi: period of interest for obsservations (2-element array), (/first, last/) years to be considered $($ default $=(/ 1900,2100 /))$

- poi_match: "contained" / "center" / "overlap" / "encloses" / "outside" (default = "overlap"), e.g., "overlap" = period of observations (given by the corresponding global attribute of the observational data file) must overlap with the "period of interest (poi)" for the data to be considered

\section{Optional GENERAL parameters}

- quantity: determines quantities to be evaluated and plotted for grading ("mean", "median" (not fully implemented yet))

- ncdf: enable to output to netCDF: either use "default" or give a full file name (default = no netCDF output)

- interpolation: flag determining regridding from simulation to observations' pressure levels: 1 = linear, $2=\log$; sign $(-/+)=$ with/without extrapolation (default: -1 or +1 will be used, depending on levels)

Optional PANELING parameters (none of them mandatory, because there are defaults)

- $\quad$ max_vert: $\max$. plots per column $($ default $=1)$

- $\quad$ max_hori: $\max$. plots per row $($ default $=1)$

- $\quad$ aux_info: string to include in outfile name $($ default $=$ period of interest $)$

Optional STYLE parameters (used by function profile_plev) 
- styleset: style, e.g., "righi15gmd" (see diag_scripts/lib/ncl/style.ncl for available styles) $($ default $=$ "DEFAULT")

- colors: override line colors (list), e.g., (/"black"/)

- dashes: overrides line styles (list), e.g., (/"0"/)

- thicks: override line thicknesses (list), e.g., $(/ 2,1,1,1,1,1,1 /) * 6$.

- annots: e.g., (/"data"/)

- FuncCode: overrides default function code for strings

- font: overrides default font (see www.ncl.ucar.edu/Document/Graphics/Resources/tx.shtml\#txFont)

Optional PLOTTING parameters (used by function profile_plev)

- $\quad$ ptop: top pressure in $\mathrm{hPa}$; "auto" = observational range from input file (default = minimum of pressure coordinate from observational data file)

- pbot: surface pressure in $\mathrm{hPa}$; "auto" = observational range from input file (default = maximum of pressure coordinate from observational data file)

- zoom: zoom x-axis range; "yes" = consider only mean/median when determining $\mathrm{x}$-axis range; "no" = use $\min / \max / 5 \% / 95 \%$ values to teremine $\mathrm{x}$-axis range; alternatively, an explicit range can be specified, e.g., $(/ 0,300 /)($ default = "yes")

- inline_legend: legend in the plot (True, False $)($ default $=$ False $)$

(3) lat_lon.ncl

Required diag_script_info attributes

- styleset: style ("DEFAULT, "righi15gmd"; see diag_scripts/lib/ncl/style.ncl for available styles)

- font: font type (see www.ncl.ucar.edu/Document/Graphics/Resources/tx.shtml\#txFont)

- mpProjection: map projection, e.g., "CylindricalEquidistant"; see http://www.ncl.ucar.edu/Document/Graphics/Resources/mp.shtml\#mpProjection for available projections)

Optional diag_script_attributes

- range_option: $0=$ as in $\mathrm{nml}, 1=$ overlapping time period

- gsnZonalMean: plot zonal mean next to map (True, False)

(4) perfmetrics_grading.ncl, perfmetrics_grading_collect.ncl, perfmetrics_main.ncl, perfmetrics_taylor.ncl, perfmetrics_taylor_collect.ncl

See "user settings" for Performance Metrics for Essential Climate Parameters.

\section{Variables}

- vmro3 (monthly mean, time level latitude longitude)

- vmrnox (monthly mean, time level latitude longitude) 
- vmrco (monthly mean, time level latitude longitude)

- $\quad \operatorname{vmrc2h} 4$ (monthly mean, time level latitude longitude)

- vmrc2h6 (monthly mean, time level latitude longitude)

- vmrc3h6 (monthly mean, time level latitude longitude)

- $\quad$ vmrc3h8 (monthly mean, time level latitude longitude)

- vmrch3 $\operatorname{coch} 3$ (monthly mean, time level latitude longitude)

- tropoz (monthly mean, time latitude longitude)

- toz (monthly mean, time latitude longitude)

- vmrco

- vmro3

\section{Observations and Reformat Scripts}

Note: (1) obs4mips data can be used directly without any preprocessing; (2) see headers of reformat scripts for non-obs4mips data for download instructions.

- Total column ozone (toz): NIWA (Bodeker et al., 2005) and GTO-ECV (Loyola and ColdeweyEgbers, 2012; Loyola et al., 2009)

Reformat scripts:

o reformat_scripts/obs/reformat_obs_NIWA.ncl

o reformat_scripts/obs/reformat_obs_GTO-ECV.ncl

- Tropospheric column ozone (tropoz): MLS/OMI (Ziemke et al., 2006)

Reformat script: reformat_scripts/obs/reformat_obs_AURA-MLS-OMI.ncl

- Ozonesonde profiles: Tilmes et al. (2012)

Reformat script: reformat_scripts/obs/reformat_obs_Tilmes.ncl

- CO surface mixing ratios: GLOBALVIEW (2010)

Reformat script: reformat_scripts/obs/reformat_obs_GLOBALVIEW.ncl

- Trace gas in-situ campaign composite data: Emmons et al. (2000)

Reformat script: reformat_scripts/obs/reformat_obs_Emmons.ncl

\section{References}

Bodeker, G. E., Shiona, H., and Eskes, H.: Indicators of Antarctic ozone depletion, Atmos. Chem. Phys., 5, 2603-2615, doi: 10.5194/acp-5-2603-2005, 2005.

Emmons, L. K., Hauglustaine, D. A., Müller, J.-F., Carroll, M. A., Brasseur, G. P., Brunner, D., Staehelin, J., Thouret, V., and Marenco, A.: Data composites of airborne observation of tropospheric ozone and its precursors, J. Geophys. Res., 105, 20497-20538, 2000. 
GLOBALVIEW-CO2: Cooperative Atmospheric Data Integration Project - Carbon Dioxide, CD-ROM, NOAA ESRL, Boulder, Colorado, available at: ftp://ftp.cmdl.noaa.gov (last access: 2 October 2014), 2010 .

Klinger, C., Quantitative evaluation of ozone and selected climate parameters in the chemistry-climate model EMAC, Master Thesis, Ludwig-Maximilians-Universität München, 2011.

Loyola, D. and Coldewey-Egbers, M.: Multi-sensor data merging with stacked neural networks for the creation of satellite long-term climate data records, EURASIP J. Adv. Sig. Pr., 2012, 1-10, doi: 10.1186/1687-6180-2012-91, 2012.

Loyola, D. G., Coldewey-Egbers, R. M., Dameris, M., Garny, H., Stenke, A., Van Roozendael, M., Lerot, C., Balis, D., and Koukouli, M.: Global long-term monitoring of the ozone layer - a prerequisite for predictions, Int. J. Remote Sens., 30, 4295-4318, doi: 10.1080/01431160902825016, 2009.

Righi, M., V. Eyring, K.-D Gottschaldt, C. Klinger, F. Frank, P. Jöckel, and I. Cionni, Quantitative evaluation of ozone and selected climate parameters in a set of EMAC simulations, Geosci. Model Dev., 8, 733-768, doi: 10.5194/gmd-8-733-2015, 2015.

Tilmes, S., Lamarque, J.-F., Emmons, L. K., Conley, A., Schultz, M. G., Saunois, M., Thouret, V., Thompson, A. M., Oltmans, S. J., Johnson, B., and Tarasick, D.: Technical Note: Ozonesonde climatology between 1995 and 2011: description, evaluation and applications, Atmos. Chem. Phys., 12, 7475-7497, doi: 10.5194/acp-12-7475-2012, 2012.

Ziemke, J. R., Chandra, S., Duncan, B. N., Froidevaux, L., Bhartia, P. K., Levelt, P. F., and Waters, J. W.: Tropospheric ozone determined from Aura OMI and MLS: Evaluation of measurements and comparison with the Global Modeling Initiative's Chemical Transport Model, J. Geophys. Res., 111, D19303, doi: 10.1029/2006JD007089, 2006.

\section{Example Plots}

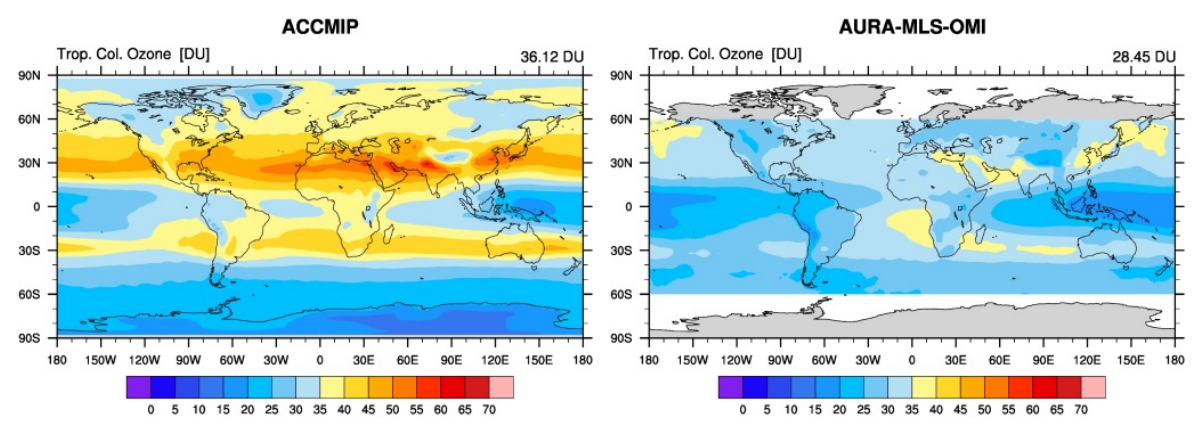


ACCMIP

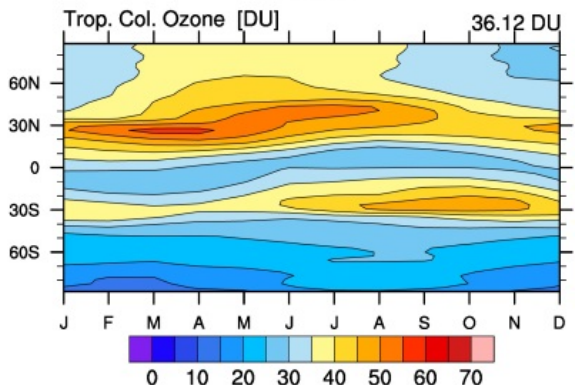

$\begin{array}{llllllll}0 & 10 & 20 & 30 & 40 & 50 & 60 & 70\end{array}$

\section{Churchill}

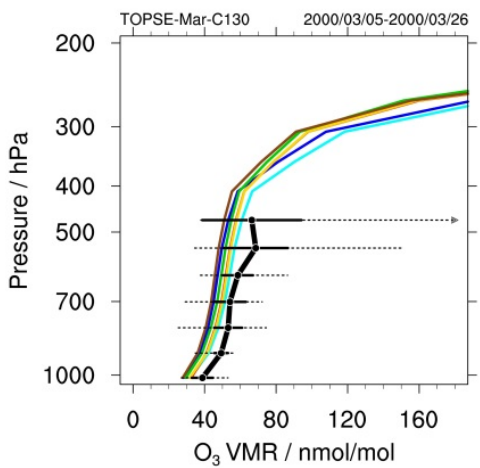

AURA-MLS-OMI
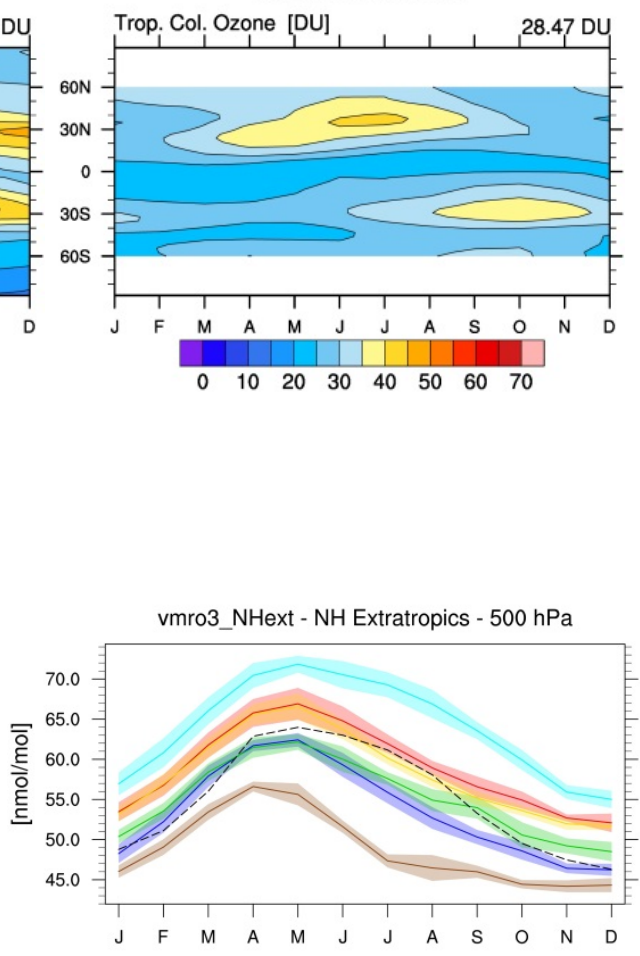


\section{Performance metrics for essential climate parameters}

\section{Overview}

The goal is to create a standard namelist for the calculation of performance metrics to quantify the ability of the models to reproduce the climatological mean annual cycle for selected "Essential Climate Variables" (ECVs) plus some additional corresponding diagnostics and plots to better understand and interpret the results. The namelist can be used to calculate performance metrics at different vertical levels (e.g., 5, 30, $200,850 \mathrm{hPa}$ as in Gleckler et al., 2008) and in four regions (global, tropics $20^{\circ} \mathrm{N}-20^{\circ} \mathrm{S}$, northern extratropics $20^{\circ}-90^{\circ} \mathrm{N}$, southern extratropics $20^{\circ}-90^{\circ} \mathrm{S}$ ). As an additional reference, we consider the Righi et al. (2015) paper.

\section{Available Namelists and Diagnostics}

Namelists are stored in $\mathrm{nml} /$

- namelist_perfmetrics_CMIP5.xml

Diagnostics are stored in diag_scripts/

- perfmetrics_grading.ncl: calculates grades according to a given metric, with different options for normalization. It requires fields precalculated by perfmetrics_main.ncl.

- perfmetrics_grading_collect.ncl: collects results from metrics previously calculated by perfmetrics_grading.ncl and passes them to the plotting functions.

- perfmetrics_main.ncl: calculates and (optionally) plots annual/seasonal cycles, zonal means, lat-lon fields and time-lat-lon fields from input monthly 2-d or 3-d ("T2M", "T3Ms") data. The calculated fields can be also plotted as difference w.r.t. a given reference model. They are also used as input to calculate grading metrics (see perfmetrics_grading.ncl).

- perfmetrics_taylor.ncl: calculates grades according to a given metric, with different options for normalization. It requires fields precalculated by perfmetrics_main.ncl.

- perfmetrics_taylor_collect.ncl: collects results from metrics previously calculated by perfmetrics_taylor.ncl and passes them to the plotting functions.

\section{User settings}

User setting files (cfg files) are stored in $\mathrm{nml} / \mathrm{cfg} \_$perfmetrics/CMIP5/

(1) perfmetrics_grading.ncl

diag_script_info attributes

- MultiModelMean: calculate multi-model mean (True, False)

- MultiModelMedian: calculate multi-model median (True, False) 
- metric: applied metric ("RMSD" = root-mean square difference, "BIAS" = mean bias, "stddev_ratio" = ratio of standard deviations of var and ref (for Taylor diagrams only), "correlation" = pattern correlation of var and ref (for Taylor diagrams only)).

- normalization: applied normalization ("mean" = normalization with mean, "median" = normalization with media, "centered_median" = substracting and dividing by the median, "stddev_mean" = normalization with substracting the mean and dividing by the standard deviation)

(2) perfmetrics_grading_collect.ncl

Required diag_script_info attributes

- label_bounds: min and max of the labelbar

- label_scale: bin width of the labelbar

- disp_values: switch on/off the grading values on the plot

Optional diag_script_info attributes

- sort: sort models in alphabetic order (excluding multi-model mean)

- title: plot title

- scale_font: scaling factor applied to the default font size

(3) perfmetrics_main.ncl

diag_script_info attributes

- plot_type: plot type ("cycle" (time), "zonal" (plev, lat), "latlon" (lat, lon), "cycle_latlon" (time, lat, lon))

- time_avg: time averaging ("monthlyclim", "seasonalclim")

- valid_fraction: required fraction of valid values

- level: vertical level (hPa, "all" for no selection; set to "all" for zonal mean plots)

• region: averaging region ("Global", "Tropics", "NH extratropics", "SH extratropics")

- grid: regridding option ("finest", "coarsest", "ref")

- draw_plots: create plots (True, False)

- plot_diff: create difference plots (only for zonal and lat-lon plots) (True, False)

- plot_stddev: plot standard deviation ("all", "none", "ref_model" or given model name)

- legend_outside: plot legend in a separate file (only for cycle plots) (True, False)

- styleset: plot style (only for cycle plots) ("CMIP5", "DEFAULT", "EMAC")

- $\quad t$ t test: calculate $t$-test for difference plots (only for zonal and lat-lon plots) (True, False)

- conf_level: confidence level for the t-test (only for zonal and lat-lon plots)

- ref_model: reference model as specified in annotations; if the attribute is a 2-element array, the first model specified is used as reference, the second model as an alternative data set.

(4) perfmetrics_taylor.ncl 


\section{Required diag_script_info attributes}

• region: averaging region ("Global", "Tropics", "NH extratropics", "SH extratropics")

• time_avg: time averaging ("monthlyclim", "seasonalclim")

- metric: selected metric (required but ignored by permetrics_taylor.ncl)

- normalization: type of metric normalization (required but ignored by permetrics_taylor.ncl)

- ref_model: reference model as specified in annotations; if the attribute is a 2-element array, the first model specified is used as reference, the second model as an alternative data set.

(5) perfmetrics_taylor_collect.ncl

diag_script_info attributes

None.

\section{Variables}

- hus (atmos, monthly mean, longitude latitude lev time)

- od550aer (aero, monthly mean, longitude latitude time)

- $\operatorname{pr}$ (atmos, monthly mean, longitude latitude time)

- rlut, rlutcs, rsut, rsutcs (atmos, monthly mean, longitude latitude time)

- ta (atmos, monthly mean, longitude latitude lev time)

- tas (atmos, monthly mean, longitude latitude time)

- ua (atmos, monthly mean, longitude latitude lev time)

- va (atmos, monthly mean, longitude latitude lev time)

- $\quad z g$ (atmos, monthly mean, longitude latitude lev time)

\section{Observations and Reformat Scripts}

Note: (1) obs4mips data can be used directly without any preprocessing; (2) see headers of reformat scripts for non-obs4mips data for download instructions.

- $\quad$ AIRS L3 (hus - obs4mips)

- CERES-EBAF (rlut, rlutcs, rsut, rsutcs - obs4mips)

- ERA-Interim (tas, ta, ua, va, zg, hus - reformat_scripts/obs/reformat_obs_ERA-Interim.ncl)

- ESACCI-AEROSOL (od550aer - reformat_scripts/obs/reformat_obs_ESACCI-AEROSOL.ncl)

- $\quad$ GPCP-SG (pr - obs4mips)

- MODIS-L3 (od550aer - obs4mips)

- NCEP (tas, ta, ua, va, zg - reformat_scripts/obs/reformat_obs_NCEP.ncl) 


\section{References}

Gleckler, P. J., K. E. Taylor, and C. Doutriaux, Performance metrics for climate models, J. Geophys. Res., 113, D06104, doi: 10.1029/2007JD008972 (2008).

Righi, M., Eyring, V., Klinger, C., Frank, F., Gottschaldt, K.-D., Jöckel, P., and Cionni, I.: Quantitative evaluation of oone and selected climate parameters in a set of EMAC simulations, Geosci. Model Dev., 8, 733, doi: 10.5194/gmd-8-733-2015 (2015).

\section{Example Plots}
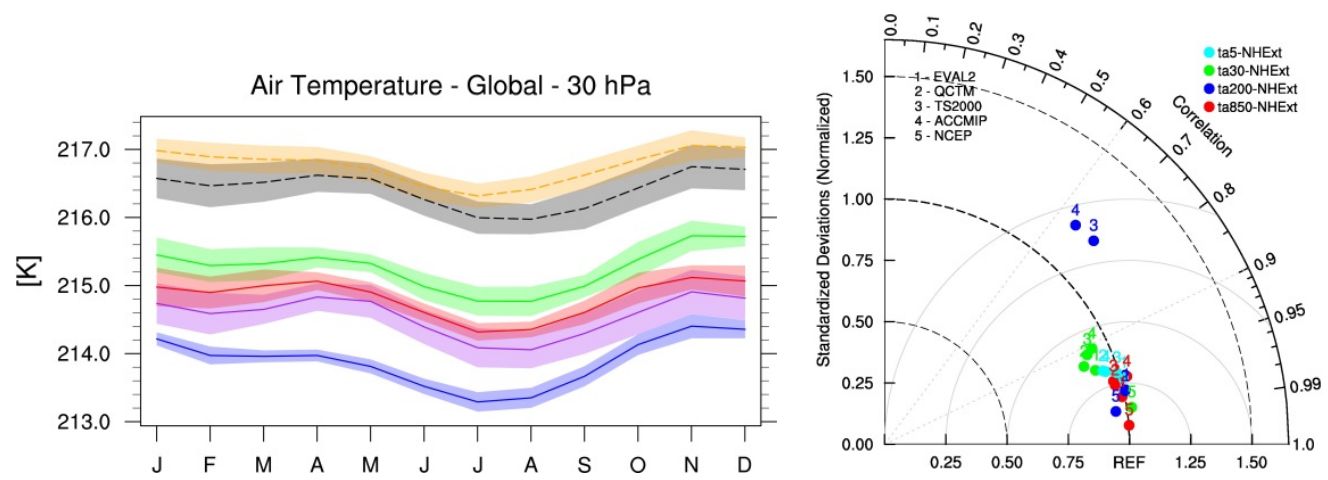

EVAL2 - SRB
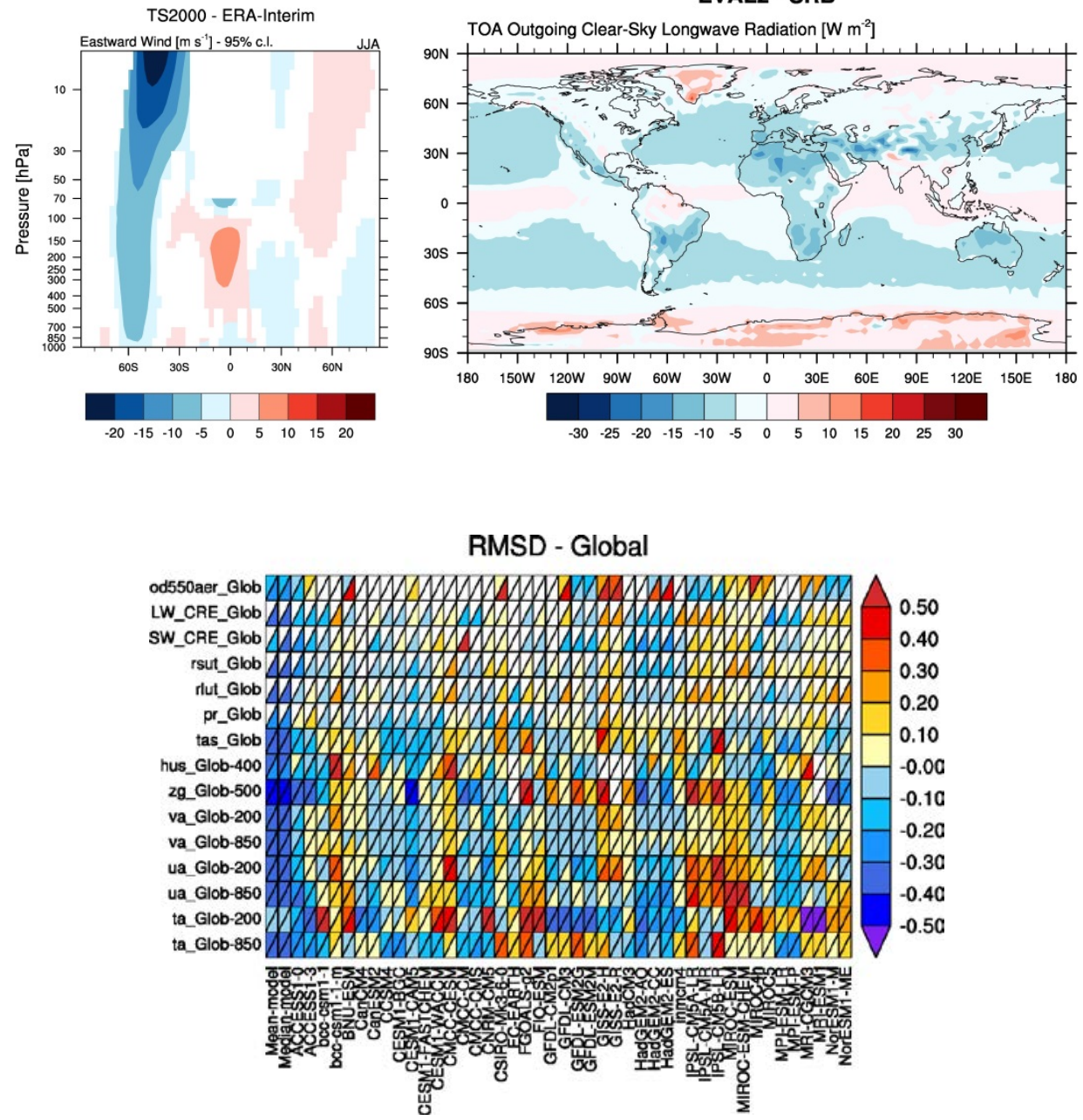


\section{South Asian Monsoon}

\section{Overview}

Three South Asian Summer Monsoon (SASM) namelists for the basic climatology, seasonal cycle, intraseasonal and inter-annual variability and key teleconnections have been implemented into the ESMValTool focusing on SASM rainfall and horizontal winds in June-September (JJAS). The goal is to provide a comprehensive overview of the basic features of the South Asian Monsoon for a certain model compared to other models, observational and reanalysis data sets for a range of processes and variables relevant to the monsoon.

\section{Available Namelists and Diagnostics}

Namelists are stored in $\mathrm{nml} /$

- namelist_SAMonsoon_AMIP.xml

- namelist_SAMonsoon_daily.xml

- namelist SAMonsoon.xml

Diagnostics are stored in diag_scripts/

- SAMonsoon_precip_basic.ncl

- SAMonsoon_precip_daily.ncl

- SAMonsoon_precip_domain.ncl

- SAMonsoon_precip_IAV.ncl

- SAMonsoon_precip_propagation.ncl

- SAMonsoon_precip_seasonal.ncl

- SAMonsoon_teleconnections.ncl

- SAMonsoon_wind_basic.ncl

- SAMonsoon_wind_IAV.ncl

- SAMonsoon_wind_seasonal.ncl

\section{User settings}

User setting files (cfg files) are stored in $\mathrm{nml} / \mathrm{cfg}$ _SAMonsoon/

(1) SAMonsoon_wind_basic.ncl

Required diag_script_info attributes

- styleset: "CMIP5", "DEFAULT"

- $\quad$ season: season in letters, e.g., "JJAS" 
- latrange_basic: min. and max. latitude of crop area (array)

- lonrange_basic: min. and max. longitude of crop area (array)

- cn_levels_mean_basic: contour levels for mean plots (array)

- cn_levels_mean_diff_basic: contour levels for mean difference plots (array)

- cn_levels_stddev_basic: contour levels for standard deviation plots (array)

- cn_levels_stddev_diff_basic: contour levels for standard deviation difference plots (array)

- my_region: monsoon region (must be set to "SA")

(2) SAMonsoon_wind_seasonal.ncl, SAMonsoon_wind_IAV.ncl

\section{Required diag_sccript_info attributes}

- styleset: "CMIP5", "DEFAULT"

- $\quad$ season: season in letters, e.g., "JJAS"

- multi_model_mean : calculate multi-model mean ("y", "n")

(3) SAMonsoon_precip_basic.ncl, SAMonsoon_precip_seasonal.ncl, SAMonsoon_precip.ncl, SAMonsoon_precip_domain.ncl, SAMonsoon_precip_basic_daily.ncl, SAMonsoon_precip_IAV.ncl, SAMonsoon_precip_propagation.ncl

Required diag_script_info attributes

- $\quad$ season: season in letters, e.g., "JJAS"

Settings specific for the precip basic plot_type

- latrange_basic: min. and max. latitude (2-element array)

- lonrange_basic: min. and max longitude (2-element array)

- cn_levels_mean_basic: contour levels for mean plots (n-element array)

- cn_levels_mean_diff_basic: contour levels for mean difference plots (n-element array)

- cn_levels_stddev_basic: contour levels for standard deviation plots (n-element array)

- cn_levels_stddev_diff_basic: contour levels for standard deviation difference plots (n-element array)

- cn_levels_stddev_norm_basic (only SAMonsoon_precip_basic_daily.ncl): contour levels for standard deviation plots (n-element array)

- cn_levels_stddev_norm_diff_basic (only SAMonsoon_precip_basic_daily.ncl): contour levels for standard deviation difference plots (n-element array)

Settings specific for the precip seasonal plot_type (SAMonsoon_precip_seasonal.ncl)

- latrange_seasonal: min. and max. latitude of crop area (2-element array)

- lonrange_seasonal: min. and max. longitude of crop area (2-element array)

- $\quad$ styleset: "CMIP5", "DEFAULT"

- multi_model_mean : calculate multi-model mean ("y", "n") 
Settings shared by the precip global domain/intensity plot_type

- summer_season: "MJJAS"

- winter_season: "NDJFM"

Settings specific for the precip global domain plot_type

- latrange_global: min. and max. latitude (2-element array)

- cn_levels_global_domain_mean: contour levels for global domain plots (array)

- cn_levels_global_domain_diff: contour levels for global domain difference plots (array)

- high_intensity_cutoff_mmday: cut-off for monsoon domain definition in $\mathrm{mm} \mathrm{day}^{-1}$

Settings specific for the precip intensity plots

- cn_levels_intensity_mean: contor levels for intensity mean plots (n-element array)

- cn_levels_intensity_diff: contor levels for intensity difference plots (n-element array)

- abs_cmap: path and filename of color map for absolute values

- diff_cmap: path and filename of color map for difference values

- my_region: monsoon region (must be set to "SA")

Settings specific for daily precipitation plots (SAMonsoon_precip_daily.ncl)

- isv_latrange: min. and max. latitude of crop area (2-element array)

- isv_lonrange: min. and max. latitude of crop area (2-element array)

- bob_latrange: min. and max. latitude of crop area for Bay of Bengal (2-element array)

- bob_lonrange $=$ min. and max. longitude of crop area for Bay of Bengal (2-element array)

- eio_latrange: min. and max. latitude of crop area for eastern equatorial Indian Ocean (2-element array)

- eio_lonrange: min. and max. longitude of crop area for eastern equatorial Indian Ocean (2element array)

- filter_min: lower limit used for filtering

- filter_max: upper limit used for filtering

- filter_weights: number of filter weights to use

Settings specific for daily propagation plots (SAMonsoon_precip_propagation.ncl)

- prop_isv_latrange: min. and max. latitude of crop area (2-element array)

- prop_isv_lonrange: min. and max. longitude of crop area (2-element array)

- prop_lag_latrange: min. and max. latitude of crop area for lag computation along longitude (2element array)

- prop_lag_lonrange: min. and max. longitude of crop area for lag computation along latitude (2element array)

- prop_ref_latrange: $\min$. and max. latitude of crop area for lag reference area (2-element array) 
- prop_ref_lonrange: min. and max. longitude of crop area for lag reference area (2-element array)

- prop_filter_mxlag: size of lag

- ihp: Lanczos switch for low/high band pass filter

- nWgt: total number of weights for Lanczos

- sigma: Lanczos sigma

- min_days: min days in filtering

- max_days: max day in filtering

- fca: start frequency to compute Lanczos weights

- fcb: end frequency to compute Lanczos weights

(4) SAMonsoon_teleconnections.ncl

\section{Required diag_script_info attributes}

- monsoon_latrange: min. and max. latitude of crop area for Indian Monsoon (2-element array)

- monsoon_lonrange: min. and max. longitude of crop area for Indian Monsoon (2-element array)

- nino34_latrange: min. and max. latitude of Niño3.4 region (2-element array)

- nino34_lonrange: min. and max. longitude of Niño3.4 region (2-element array)

- tele_latrange: min. and max. latitude of crop area for overview area (2-element array)

- tele_lonrange: min. and max. longitude of crop area for overview area (2-element array)

- cn_levels_mean_basic: contour levels for mean plot (n-element array)

- cn_levels_mean_diff_basic: contour levels for mean difference plot (n-element array)

- cn_levels_stddev_basic: contour levels for standard deviation plot (n-element array)

- cn_levels_stddev_diff_basic: contour levels for standard deviation difference plot (n-element array)

\section{Variables}

- $\operatorname{pr}$ (atmos, daily/monthly, longitude latitude time)

- ts (atmos, monthly, longitude latitude time)

- ua (atmos, monthly, longitude latitude lev time)

- va (atmos, monthly, longitude latitude lev time)

\section{Observations and Reformat Scripts}

Note: (1) obs4mips data can be used directly without any preprocessing; (2) see headers of reformat scripts for non-obs4mips data for download instructions.

- ERA-Interim (ua, va, pr - reformat_scripts/obs/reformat_obs_ERA-Interim.ncl, reformat_obs_ERAInterim_precip.ncl) 
- NCEP (ua, va - reformat_scripts/obs/reformat_obs_NCEP.ncl)

- $\quad$ TRMM-L3 (pr, monthly means - obs4mips)

- TRMM_3B42 (pr, daily means - reformat_scripts/obs/reformat_obs_TRMM_3B42_daily.ncl)

- HadISST (ts - reformat_scripts/obs/reformat_obs_HadISST.ncl)

- GPCP-1DD (pr, daily means - obs4mips)

- $\quad$ GPCP-SG (pr, monthly means - obs4mips)

- MERRA (pr - reformat_scripts/obs/reformat_obs_MERRA.bash)

- CMAP (pr - reformat_scripts/obs/reformat_obs_CMAP.ncl)

\section{References}

Sperber, K. R., et al., The Asian summer monsoon: an intercomparison of CMIP5 vs. CMIP3 simulations of the late 20th century, Clim Dyn (2013) 41:2711-2744, doi: 10.1007/s00382-012-1607-6, 2012.

Lin, Jia-Lin, Klaus M. Weickman, George N. Kiladis, Brian E. Mapes, Siegfried D. Schubert, Max J. Suarez, Julio T. Bacmeister, Myong-In Lee, 2008: Subseasonal Variability Associated with Asian Summer Monsoon Simulated by 14 IPCC AR4 Coupled GCMs. J. Climate, 21, 4541-4567. doi: http://dx.doi.org/10.1175/2008JCLI1816.1.

Webster, P. J., and S.Yang, 1992: Monsoon and ENSO: Selectively interactive systems. Quart. J. Roy. Meteor. Soc., 118, 877-926. (Webster-Yang dynamical monsoon index)

Goswami, B. N., B. Krishnamurthy, and H. Annamalai, 1999: A broad-scale circulation index for interannual variability of the Indian summer monsoon. Quart. J. Roy. Meteor. Soc., 125, 611-633. (Goswami dynamical monsoon index)

Wang, B., and Z. Fan, 1999: Choice of south Asian summer monsoon indices. Bull. Amer. Meteor. Soc., 80, 629-638. (Wang-Fan dynamical monsoon index)

Wang B., J. Liu, H. J. Kim, P. J. Webster, and S. Y. Yim, Recent change of global monsoon precipitation (1979-2008), Climate Dynamics, doi: 10.1007/s00382-011-1266-z, 2011. (Intensity/Monsoon domain reference) 


\section{Example Plots}

JJAS-mean of Precipitation Rate
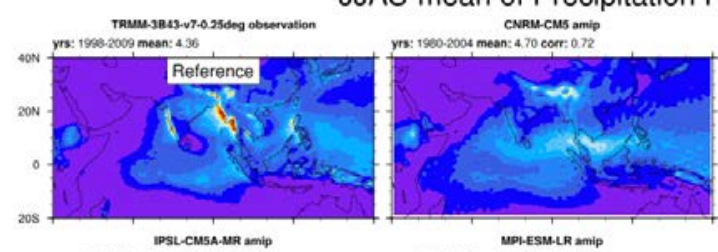

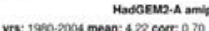

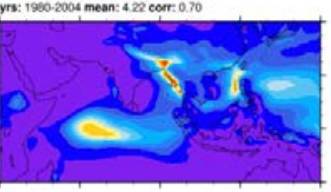

MPl.ESMLLR amip
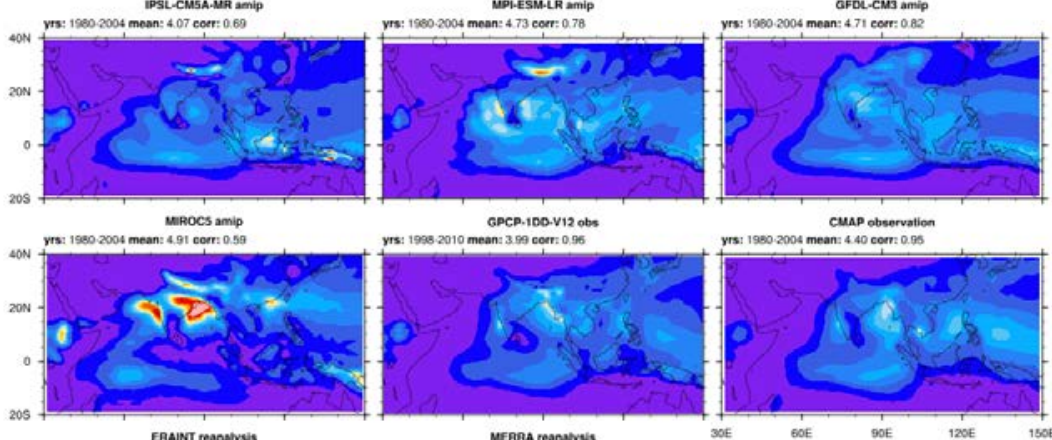

Yrs: 19982010 mean: 3.09 corvin: 0.96
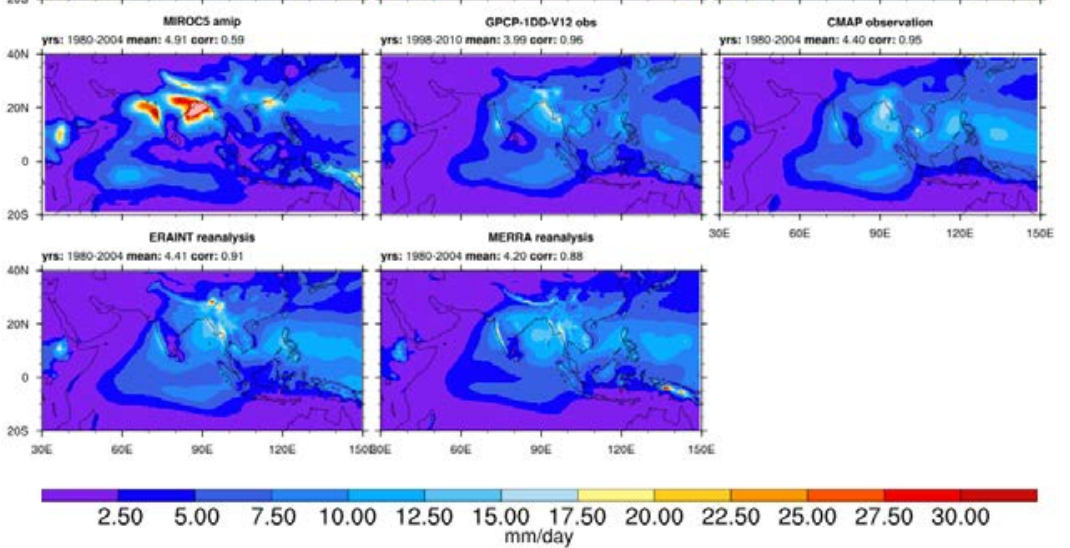

\section{JJAS-mean of Precipitation}
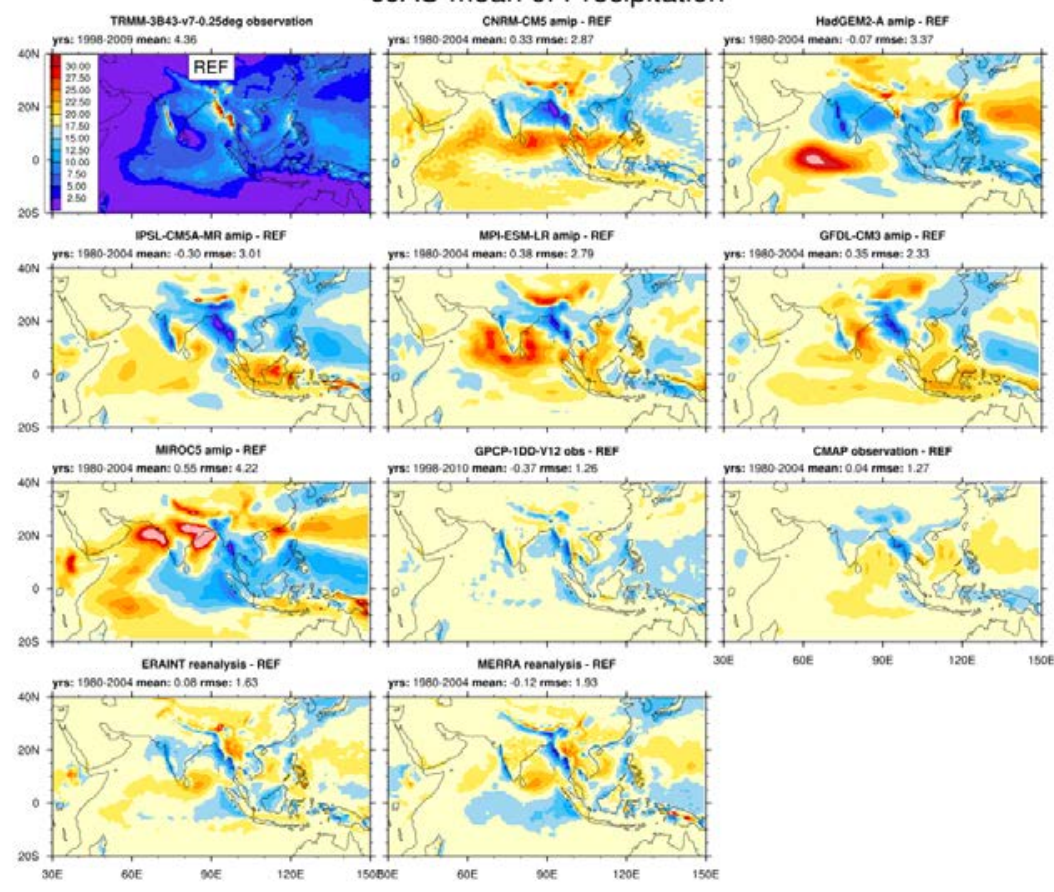

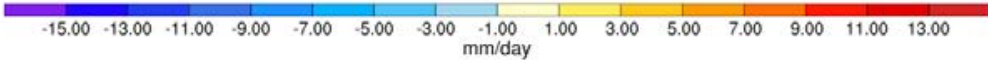




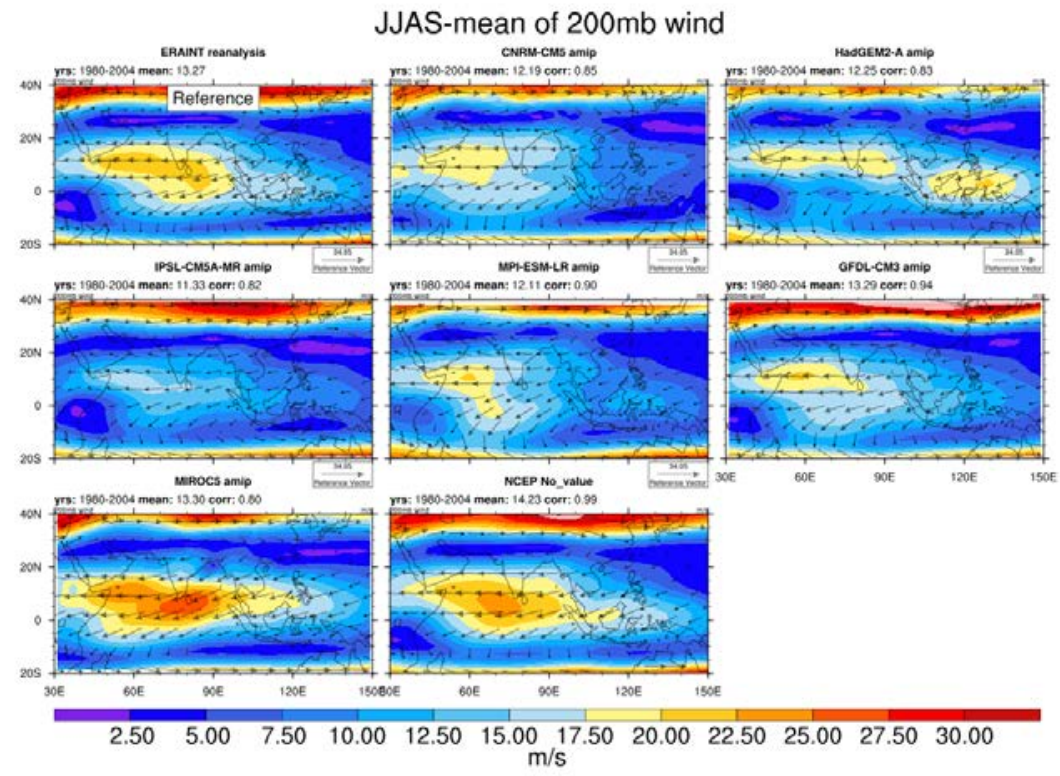

(1) JJAS-mean of TRMM-3B43-v7-0.25deg observation yrs: $1998-2009$ mean: $4.36 \quad$ Precipitation Rate

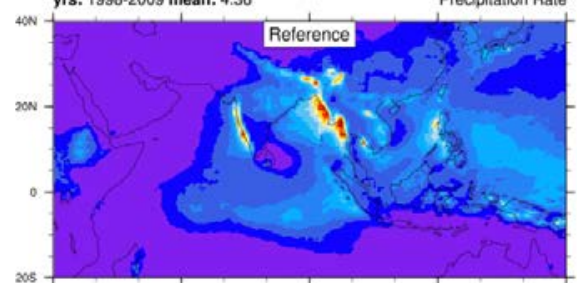
$\mathrm{mm} /$ day
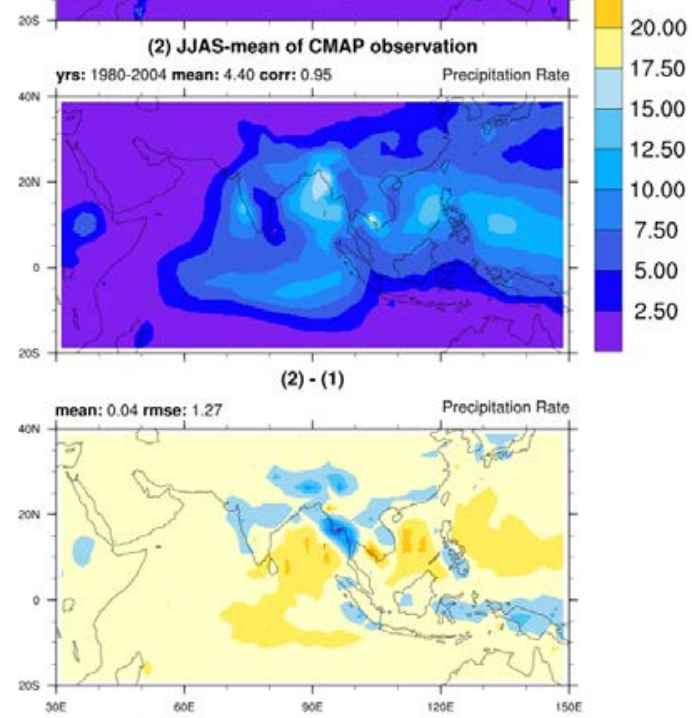

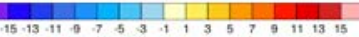


Monsoon Global Domain: Model minus Reference
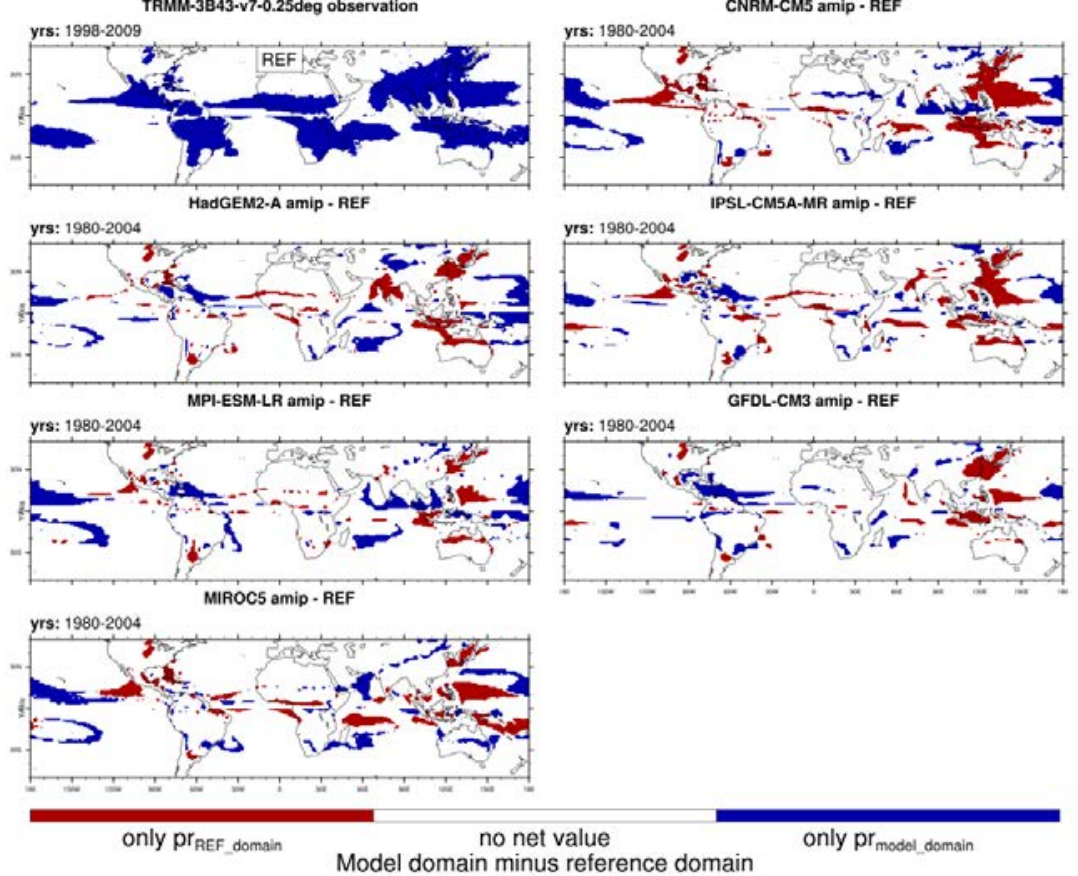

JJAS-mean precip-Nino3.4 SST teleconnection, mean plots

TRMM-3B43-v7-0.25deg/MOHC-HadISST

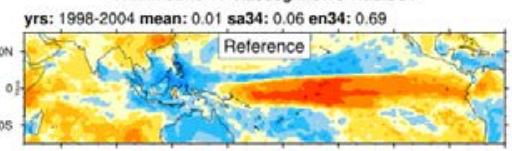

HadGeM2-A amip

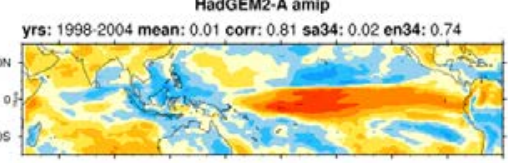

MPI-ESM-LR amip

yrs: $1998-2004$ mean: -0.00 corr: 0.79 sa34: -0.02 en34: 0.67

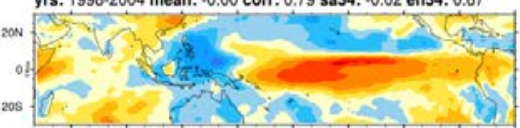

MiRoc5 amip

yrs: $1998-2004$ mean: 0.03 corr: 0.74 sa34: 0.08 en34: 0.79

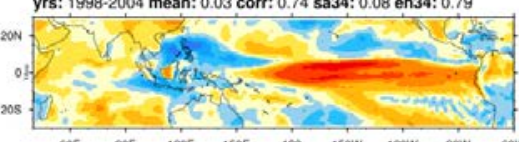

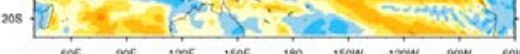

$\begin{array}{lllllllllllll}-1.00 & -0.85 & -0.70 & -0.55 & -0.40 & -0.25 & -0.10 & 0.05 & 0.20 & 0.35 & 0.50 & 0.65 & 0.80\end{array}$

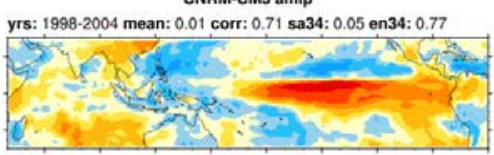

IPSL-CM5A-MR amip

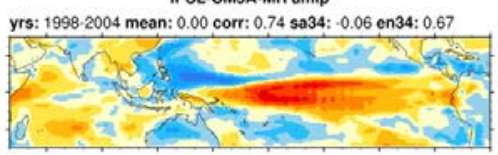

GFDL-CM3 amip

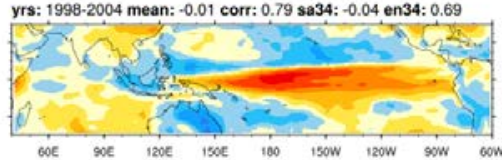
(1) 
IAV Precipitation (JJAS)

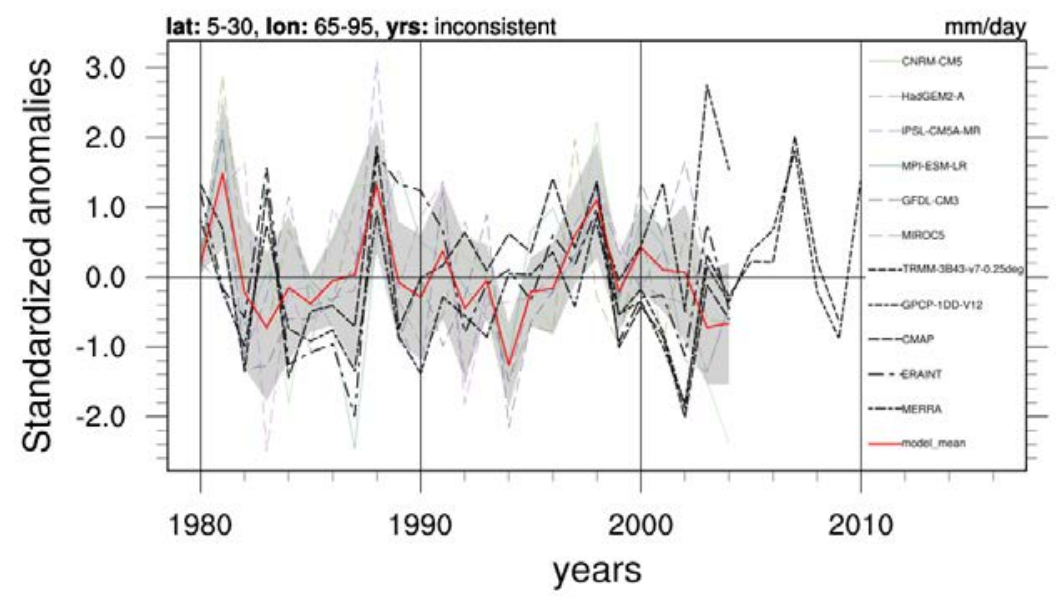

JJAS-rp-timelat lag-lat propagation of filtered daily Precip anom

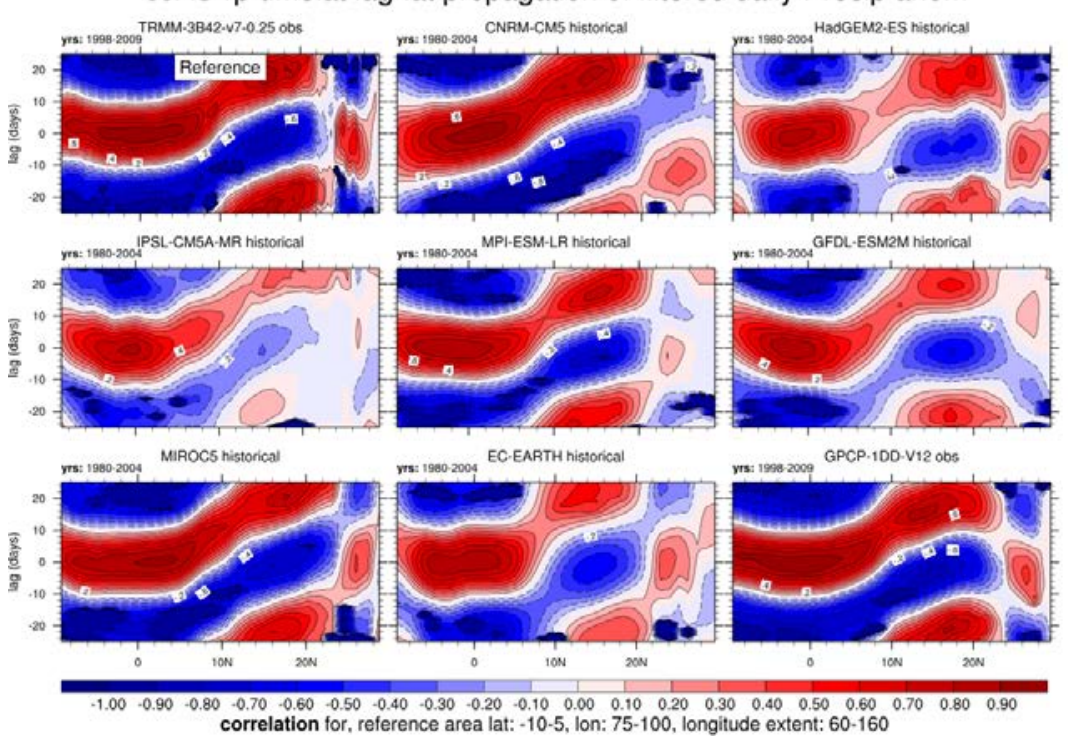

Intraseasonal JJAS-stddev filtered 30-50 daily rainfall
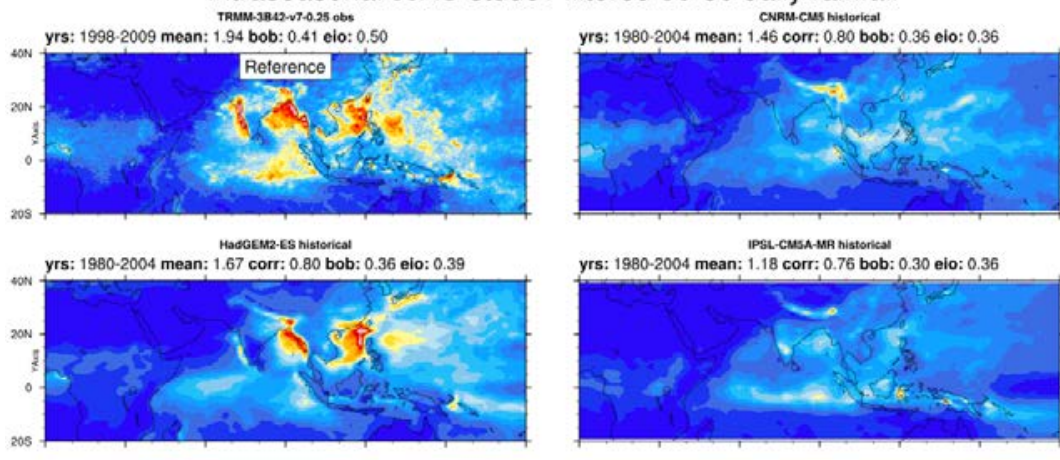

yrs: 1980-2004 mean: 1.18 corr: 0.76 bob: 0.30 elo: 0.36

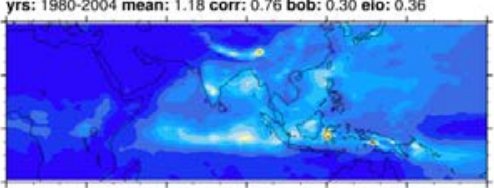

Mph LESMLA LA hastorical
1.67 corr: 0.83 bob: 0.37 eio: 0.34

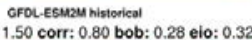

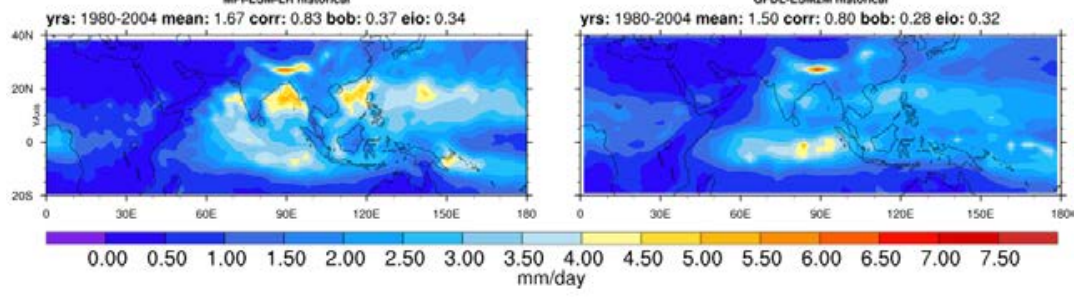




\section{Sea ice}

\section{Overview}

The sea ice diagnostics cover sea ice extent and concentration, but work is underway to include other variables and processes in future releases of the ESMValTool. Current diagnostics include time series of September (Arctic) and March (Antarctic) sea ice extent calculated as the total area $\left(\mathrm{km}^{2}\right)$ of grid cells with sea ice concentrations (sic) of at least $15 \%$. Also included are the seasonal cycle of sea ice extent, polar stereographic contour and polar contour difference plots of Arctic and Antarctic sea ice concentrations.

\section{Available Namelists and Diagnostics}

Namelists are stored in $\mathrm{nml} /$

- namelist_SeaIce.xml

Diagnostics are stored in diag_scripts/

- SeaIce_tsline.ncl: time series line plots of total sea ice area and extent (accumulated) for northern and southern hemispheres with optional multi-model mean and standard deviation. One value is used per model per year, either annual mean or the mean value of a selected month.

- SeaIce_ancyc.ncl: as SeaIce_tsline.ncl, but for the annual cycle (multi-year monthly mean values).

- SeaIce_polcon.ncl: polar stereographic plots of sea ice concentration (= sea ice area fraction) and extent (grid cells with a sea ice concentration of at least 15\%) for individual models or observational data sets, for Arctic and Antarctic regions with flexible paneling of the individual plots. The edges of sea ice extent can be highlighted via an optional red line.

- SeaIce_polcon_diff.ncl: polar stereographic plots of sea ice area concentration difference between individual models and reference data (e.g., an observational data set) for both Arctic and Antarctic with flexible paneling of the individual plots. All data are regridded to a common grid $\left(1^{\circ} \mathrm{x} 1^{\circ}\right)$ before comparison.

\section{User settings}

User setting files (cfg files) are stored in $\mathrm{nml} / \mathrm{cfg} \_$SeaIce/

- region: label of region to be plotted ("Arctic", "Antarctic"); make sure to specify correct observational data for the selected region in the sea ice namelist.

- $\quad$ month: "A" = annual mean, "3" = March (Antarctic), "9" = September (Arctic)

- $\quad$ styleset: "CMIP5", "DEFAULT"

Settings specific to SeaIce_polcon, SeaIce_polcon_diff, SeaIce_ancyc

- range_option: $0=$ use each model's whole time range as specified in namelist, $1=$ use only intersection of all time ranges

Setting specific to SeaIce_tsline.ncl and SeaIce_ancyc.ncl 
- multi_model_mean: plots multi-model mean and standard deviation ("y", "n")

- EMs_in_lg: create legend label for each individual ensemble member (True, False)

Settings specific to SeaIce_polcon.ncl and SeaIce_polcon_diff.ncl

- contour_extent: draw a red contour line for sic extent in polar stereographic plots ("y", "n")

- max_vert: max. number of rows on a panel page (vertical)

- max_hori: max. number of columns on a panel page (horizontal)

- max_lat: Antarctic plotted from $90^{\circ} \mathrm{S}$ up to this latitude

- min_lat: Arctic plotted from $90^{\circ} \mathrm{N}$ up to this latitude

- PanelTop: tune to get full title of uppermost row $(1=$ no top margin, default $=0.99)$

Settings specific to SeaIce_polcon_diff.ncl

- ref_model: reference model, as specified in annotations; if this string is not found, the routine will print a list of valid strings before stopping

- grid_min: $\min$. contour value (default $=-1.0)$

- grid_max: max. contour value (default $=1.0)$

- grid_step: step between contours (default $=0.2)$

- grid_center: value to center the color bar $($ default $=0.0)$

\section{Variables}

- $\quad$ sic (sea ice, monthly mean, longitude latitude time)

\section{Observations and Reformat Scripts}

Note: (1) obs4mips data can be used directly without any preprocessing; (2) see headers of reformat scripts for non-obs4mips data for download instructions.

- National Snow and Ice Data Center (NSIDC)

Reformat script: reformat_scripts/obs/reformat_obs_NSIDC.ncl

- Hadley Centre Sea Ice and Sea Surface Temperature data set (HadISST)

Reformat script: reformat_scripts/obs/reformat_obs_HadISST.ncl

\section{References}

Bräu, M.: Sea-ice in decadal and long-term simulations with the Max Planck Institute Earth System Model, Bachelor thesis, LMU, 2013.

Hübner, M.: Evaluation of Sea-ice in the Max Planck Institute Earth System Model, Bachelor thesis, LMU, 2013. 


\section{Example Plots}
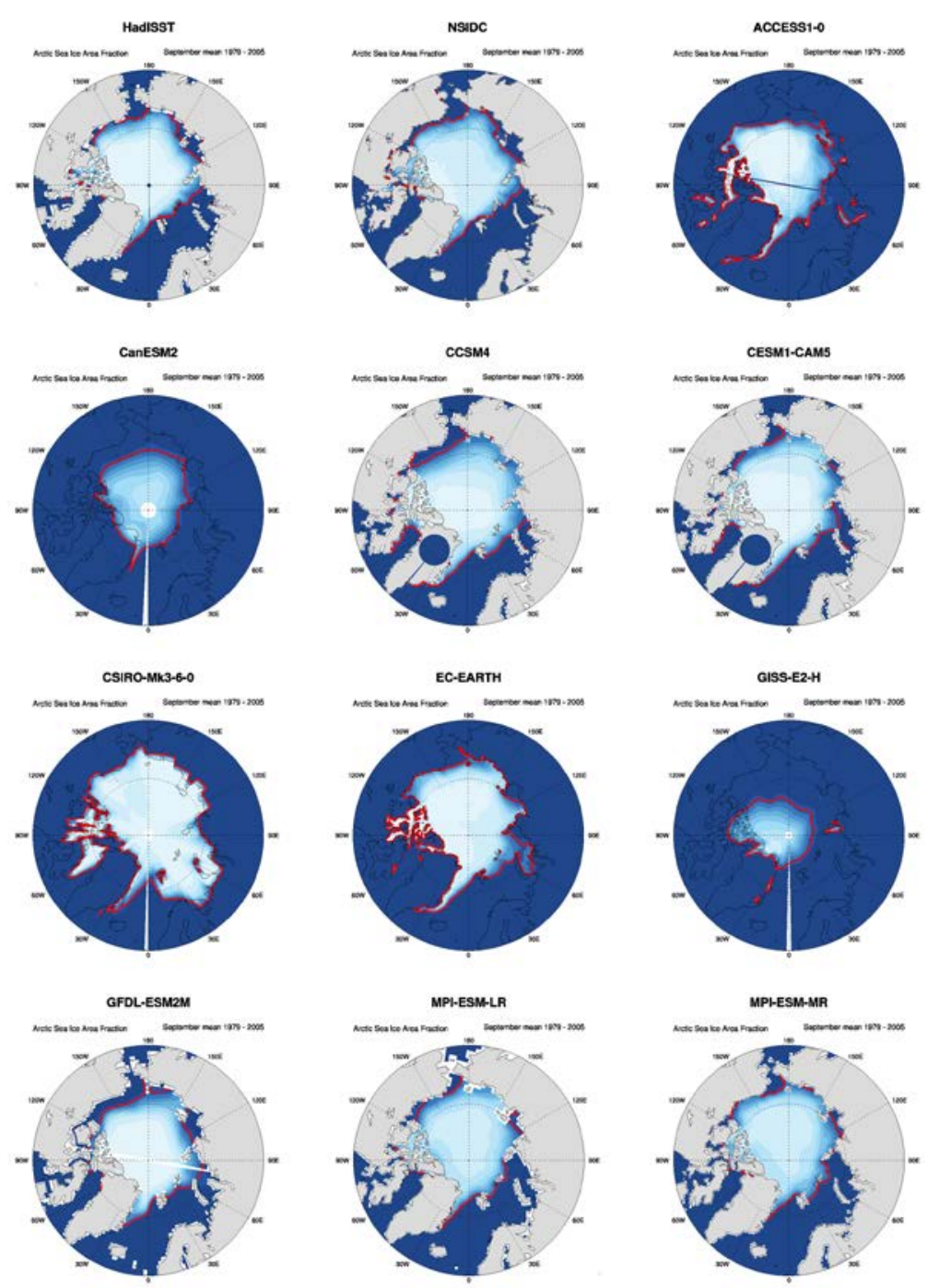

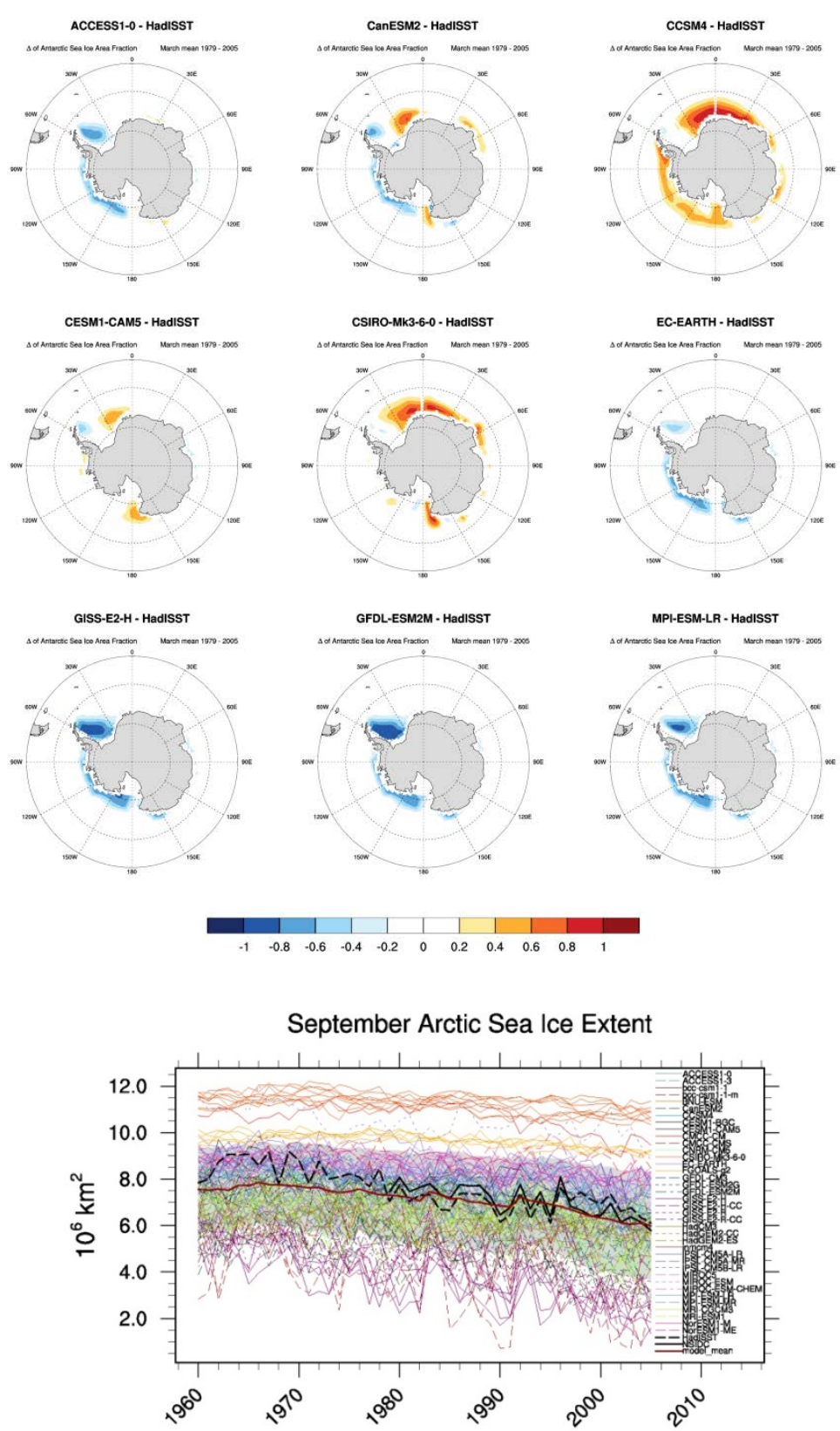

Multi-year monthly mean Antarctic Sea Ice Extent

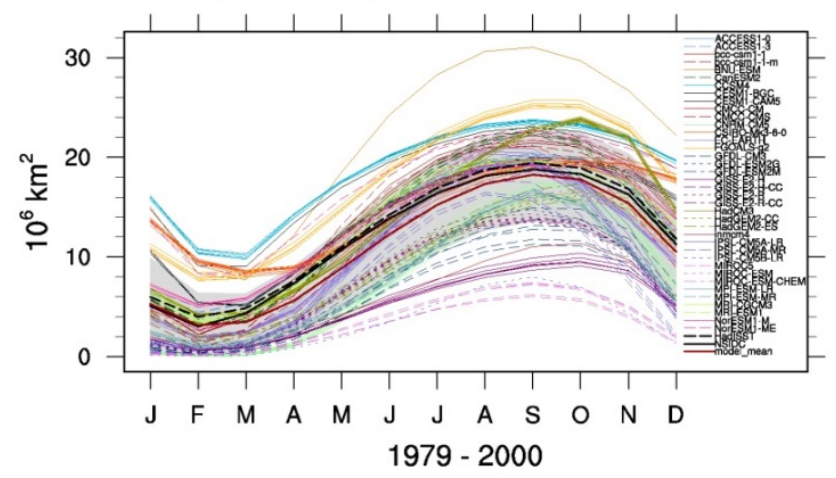




\section{Southern Hemisphere}

\section{Overview}

The diagnostics compare model estimates of cloud, radiation and surface turbulent flux variables with suitable observational data sets. Seasonal mean maps of TOA, total and clear-sky, outgoing short wave (SW) and long wave (LW) radiation are constructed for the Southern Ocean region $\left(30^{\circ} \mathrm{S}-65^{\circ} \mathrm{S}\right)$ comparing available model data with CERES-EBAF satellite observations (Loeb et al., 2005). Absolute values, model to satellite differences and total minus clear sky flux values are plotted through standard namelists. Combined with TOA radiation, seasonal mean maps of total cloud cover, vertically integrated cloud liquid water and cloud ice are also plotted with CloudSat data (Stephens et al., 2002) providing an observational constraint. Finally, seasonal mean maps of surface sensible and latent heat fluxes over the same region compare model estimates with the Woods Hole OAflux observations ( $\mathrm{Yu}$ et al., 2008). For each of these quantities (TOA radiation, cloud and surface flux variables) climatological annual cycles are constructed using zonal means averaged separately over 3 latitude bands: (i) $30^{\circ} \mathrm{S}-65^{\circ} \mathrm{S}$, the entire Southern Ocean, (ii) $30^{\circ} \mathrm{S}-45^{\circ} \mathrm{S}$, the subtropical Southern Ocean, and (iii) $45^{\circ} \mathrm{S}-65^{\circ} \mathrm{S}$, the mid-latitude Southern Ocean. Finally, annual means of each variable (models and observations) are constructed as zonal means, plotted over $30^{\circ} \mathrm{S}-$ $65^{\circ} \mathrm{S}$, and as latitudinal means (over $30^{\circ} \mathrm{S}-65^{\circ} \mathrm{S}$ ) plotted around a longitude circle.

\section{Available Namelists and Diagnostics}

Namelists are stored in $\mathrm{nml} /$

- namelist_SouthernHemisphere.xml

Diagnostics are stored in diag_scripts/

- SouthernHemisphere.py

- SouthernHemisphere_scatter.py

\section{User settings}

User setting files (cfg files) are stored in $\mathrm{nml} / \mathrm{cfg} \_$SouthernHemisphere/

(1) SouthernHemisphere.py and SouthernHemisphere_scatter.py

Diag_script_info attributes

General options for all diagnostics

- plot_clouds: True, False

- plot_fluxes: True, False

- plot_radiation: True, False

- plot_scatter: True, False

- plot_background_grid: True, False 
- plot_total_cover: True, False

- plot_liquid_path: True, False

- plot ice path: True, False

- plot_optical_depth: True, False

- plot_flux_maps: True, False

- plot_radiation_maps: True, False

Common sub keys for all diagnostics (plot_): X_maps, X_averages, sub_areas

- plot_lat_averages: True, False

- plot_lon_averages: True, False

- plot_monthly_averages: True, False

- plot_sub_areas: True, False

- mask_unwanted_values: mask values (e.g., missing values) exceeding "mask_limit_low" and "mask_limit_high" (True, False)

- mask_limit_low: lower threshold used for creating a mask (if "mask_unwanted_values" = True)

- mask_limit_high: upper threshold used for creating a mask (if "mask_unwanted_values" = True)

Configuration for Southern Hemisphere maps and plots. All plots will be generated for all areas but only monthly averages for sub_areas.

- areas: name of area(s), defined separately for each area (see below), e.g., default

- sub_areas: name of sub-area(s), defined separately for each sub-area (see below), e.g., northern southern

- scatter_areas: cloud vs radiation scatter plot areas; areas must be defined separately (see below), e.g., default

- seasons: which months to plot for each contour and lat/lon mean plots (e.g., DJF MAM JJA $\mathrm{SON}$ ); each season has to be defined separately (see below)

Definition of each area, e.g., [SouthernHemisphere_default]

- lat_min: min. latitude

- lat_max: max. latitude

- lon_min: min. longitude

- lon_max: max. longitude

- stride: color difference interval (how many units per color/shade); if set to 0 , the stride is calculated automatically using "maxshades" (max. number of colors/shades)

- maxshades: max. number of colors/shades

The contour_limits_* are contour map limits and are given by 3 or 5 integers (min, max, diff, [dev_min, dev_max]). The min and max values define the limits for model maps (and the clear sky (cs) variant). 
The diff value gives a range [-diff, diff] for difference maps of model - obs (and cs). The last range [dev_min, dev_max] is the range for model/obs - model/obs (cs).

- \# hfls, hfss (latent, sensible heat); rlut, rsut (long, shortwave radiation).

- contour_limits_clt: contour limits for total cloud cover

- contour_limits_clivi: contour limits for ice water path

- contour_limits_clwvi: contour limits for total condensed water path

- contour_limits_hfls: contour limits for latent heat

- contour_limits_hfss: contour limits for sensible heat

- contour_limits_rlut: contour limits for longwave radiation

- contour_limits_rsut: contour limits for shortwave radiation

- contour_limits_rlds: contour limits for surface downwelling longwave radiation

- contour_limits_rsds: contour limits for surface downwelling shortwave radiation

Color maps for map plots. You may change the maps as you will, just google "python matplotlib colormaps" for examples. Color maps can be inverted by adding ' $r$ ' at the end.

- colourmap_clouds: e.g., Blues

- colourmap_model: e.g., RdYlGn_r

- colourmap_diff: e.g., jet

- colourmap_dev: e.g., cool hot_r

Definition of sub areas, e.g., [SouthernHemisphere_northern]

- lat_min: min. latitude

- lat_max: max. latitude

- lon_min: min. longitude

- lon_max: max. longitude

Definition of months covered by each season -1 is January and so forth, e.g., [SouthernHemisphere_season_DJF]

- season_months: e.g., 1212

Configuration for cloud vs radiation scatter plots

- lat_min: min. latitude

- lat_max: max. latitude

- lon_min: min. longitude

- lon_max: max. longitude

- points: number of bins 


\section{Variables}

- clt: total cloud cover fraction (atmos, monthly mean, time latitude longitude)

- clivi: cloud ice water path (atmos, monthly mean, time latitude longitude)

- clwvi: total condensed water path (atmos, monthly mean, time latitude longitude)

- hfls: latent heat flux (atmos, monthly mean, time latitude longitude)

- hfss: sensible heat flux (atmos, monthly mean, time latitude longitude)

- rlut: top of atmosphere outgoing longwave radiation (atmos, monthly mean, time latitude longitude)

- rlutcs: top of atmosphere outgoing clear-sky longwave radiation (atmos, monthly mean, time latitude longitude)

- rsut: top of atmosphere outgoing shortwave radiation (atmos, monthly mean, time latitude longitude)

- rsutcs: top of atmosphere outgoing clear-sky shortwave radiation (atmos, monthly mean, time latitude longitude)

\section{Observations and Reformat Scripts}

Note: (1) obs4mips data can be used directly without any preprocessing; (2) see headers of reformat scripts for non-obs4mips data for download instructions.

- CERES-EBAF (obs4mips)

- WHOI-OAFlux

Reformat script: reformat_scripts/obs/reformat_obs_OAFlux.ncl

- CloudSat-L3

Reformat script: reformat_scripts/obs/reformat_obs_cloudsat.bash

- MODIS-L3-C6

Reformat script: reformat_scripts/obs/reformat_obs_modis.ncl

\section{References}

None. 


\section{Example Plots}

TOA outgoing longwave radiation for months: DJF
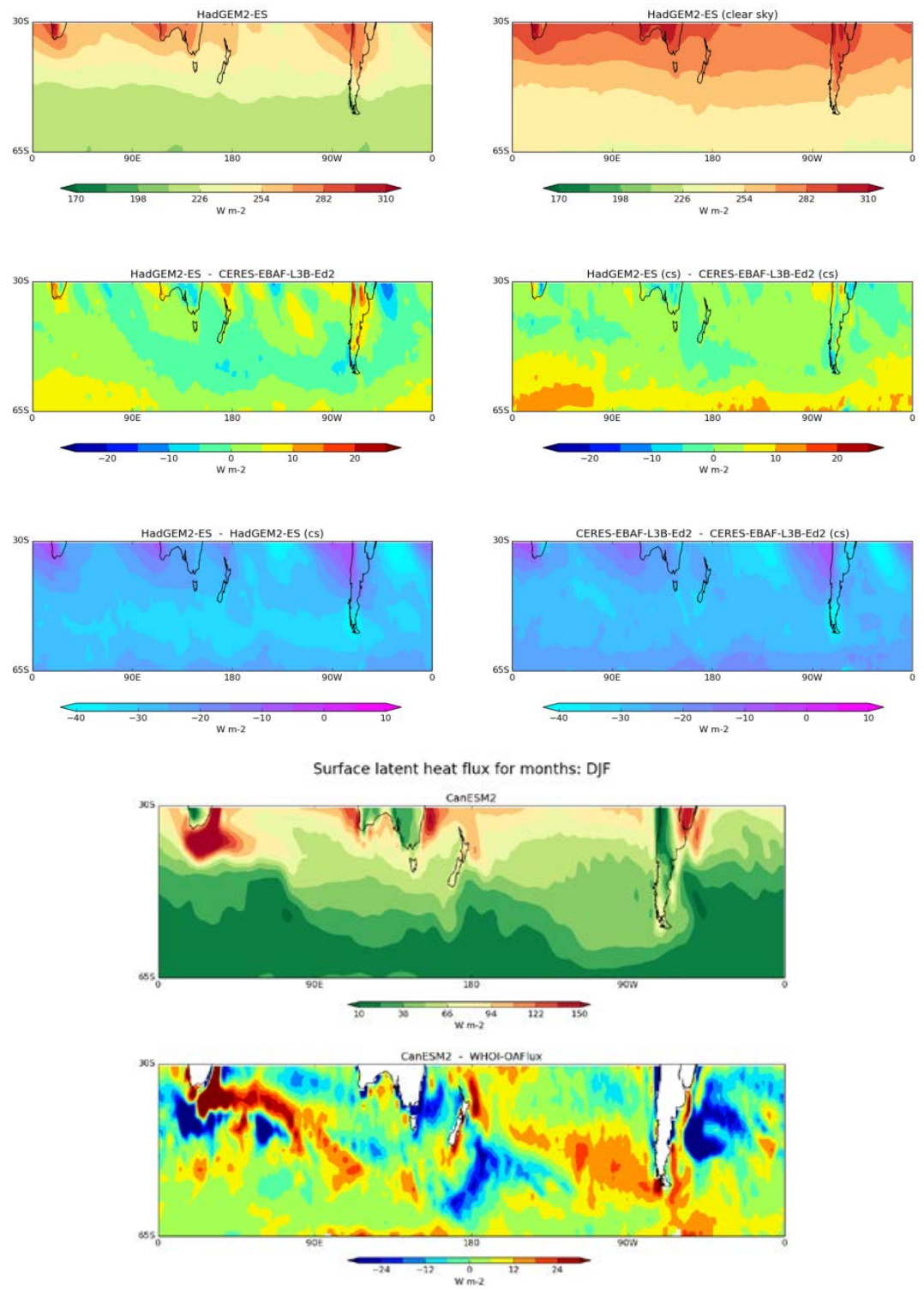

TOA clear sky outgoing longwave radiation (Ions [0:360])
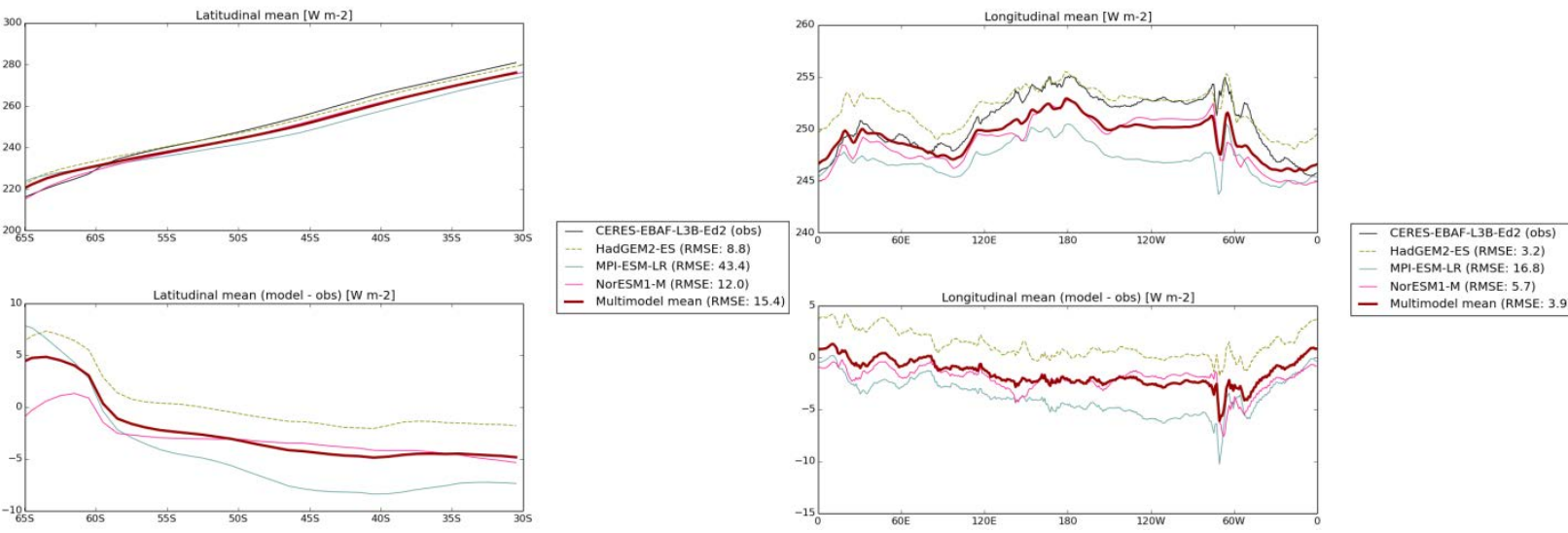

TOA clear sky outgoing longwave radiation (lats [65S:30S]) - Multimodel mean (RMSE: 3.9 . 
TOA clear sky outgoing longwave radiation (lats [65S:30S], Ions [0:360])

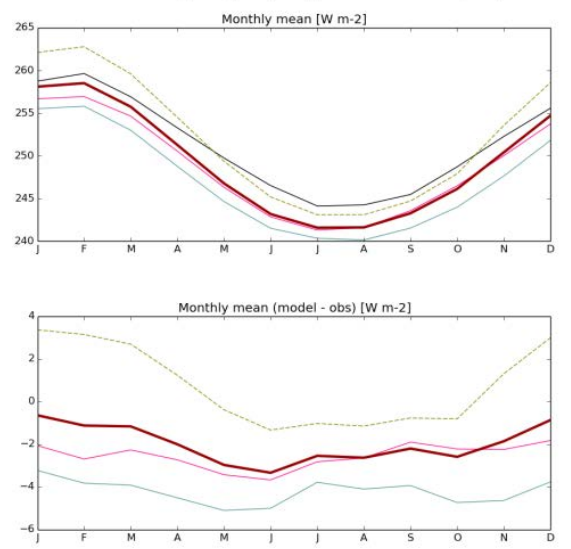

- CERES-EBAF-L3B-Ed2 (obs)
- HadGEM2-ES (RMSE: 3.8 )
- MPl-ESM-LR (RMSE: 18.0)

- NorESM1-M (RMSE: 6.8) 


\section{Southern Ocean}

\section{Overview}

These diagnostics include polar stereographic (difference) maps to compare the monthly/annual mean sea surface temperature, salinity and wind stress from ESMs with ERA-Interim data. Furthermore, there are scripts to plot the differences in the area mean vertical profiles of temperature and salinity between models and data from the World Ocean Atlas (Antonov et al., 2010; Locarnini et al., 2010). The ocean mixed layer thickness from models can be compared with that obtained from the Argo floats (Dong et al., 2008), again using polar stereographic (difference) maps. Finally, the Antarctic Circumpolar Current strength, as measured by water mass transport through the Drake Passage, is calculated using the same method as in the CDFTOOLS package (CDFtools). This diagnostic can be used to calculate the transport through other section as well, but is only available for EC-Earth/NEMO output for which all grid information is available.

\section{Available Namelists and Diagnostics}

Namelists are stored in $\mathrm{nml} /$

- namelist_SouthernOcean.xml

Diagnostics are stored in diag_scripts/

- SouthernOcean_polcon.ncl: create polar stereographic plots for ocean mixed layer thickness, sea surface salinity and sea surface temperature.

- SouthernOcean_polcon_diff.ncl: create polar stereographic plots of the difference between individual models and reference data for ocean mixed layer thickness, sea surface temperature and eastward and northward wind stress. All data are regridded to a common grid using the ESMF regridding software.

- SouthernOcean_vector_polcon_diff.ncl: create polar stereographic contour plots of the difference between individual model data and reference data similar to SouthernOcean_polcon_diff.ncl, but on top plots vectors (magnitude and direction) for both the individual models and the reference data. Currently it is used for wind stress, but it should be possible to use it for other variables with $\mathrm{u}$ and $\mathrm{v}$ components as well. All data are regridded to a common grid using the ESMF regridding software.

- SouthernOcean_areamean_vertconplot.ncl: calculate the average sea water salinity and temperature over a subdomain from model data and create a Hovmoller-like diagram with time and depth on the axes. All data are regridded to a common grid using the ESMF regridding software.

- SouthernOcean_transport.ncl: calculate the sea water volume transport across a section from the variables uo and vo using a similar approach as is done in the CDFTOOLS package. Currently only available for EC-Earth/Nemo output as the calculations are performed using uo and vo on a staggered grid and the grid dimensions of the $\mathrm{u}$ and $\mathrm{v}$ grids are required.

\section{User settings}

User setting files (cfg files) are stored in $\mathrm{nml} / \mathrm{cfg} \_$SouthernOcean/ 
(1) SouthernOcean_polcon.ncl, SouthernOcean_polcon_diff.ncl, SouthernOcean_vector_polcon_diff.ncl

- region: "Antarctic" (entire hemisphere will be evaluated)

- $\quad$ month: "A" (A = annual mean, 3 = March, 9 = September $)$

• $\quad$ styleset: "CMIP5", "DEFAULT"

- max_vert: max. number of rows on a panel page (vertical)

- max_hori: max. number of columns on a panel page (horizontal)

- grid_min: $\min$. contour value (default $=-1.0)$

- grid_max: max. contour value (default $=1.0)$

- grid_step: step between contours $($ default $=0.2)$

- colormap: color table (from NCL distribution)

- PanelLabelBar: use single label bar per page (True, False)

- $\quad$ showunits: display units in figure title (True, False)

- range_option: $0=$ use each model's time range specified in namelist, $1=$ use only intersection of all time ranges

Setting specific to SouthernOcean_vector_polcon_diff.ncl

- vcmagnitude: magnitude of vectors (larger value = shorter arrows)

- vemindist: controls density of vectors (larger value = less arrows)

- vccolormod: color of vectors for current model

- vccolorref: color of vectors for reference model

(2) SouthernOcean_areamean_vertconplot.ncl

Required diag_script_info attributes

- region: name of region ("Southern Ocean")

- West: western boundary of area

- East: eastern boundary of area

- South: southern boundary of area

- North: northern boundary of area

- $\quad$ styleset: plot style ("CMIP5", "DEFAULT")

Optional diag_script_info attributes

- grid_min: min. for plot scale

- grid_max: max. for plot scale

- grid_step: step size for plot scale

- colormap: e.g., WhiteBlueGreenYellowRed, rainbow

- dbg_plots: create additional plots for debugging purposes (True, False) 
(3) SouthernOcean_transport.ncl

Required diag_script_info attributes

- $\quad$ styleset: plot style ("CMIP5", "DEFAULT")

- lon1: longitude of start of section

- lat1: latitude of start of section

- lon2: longitude of end of section

- lat2: latitude of end of section

- section_name: name used in title, e.g., "Drake passage"

\section{Variables}

- hfds (atmos, monthly mean, longitude latitude time) $=\mathrm{hfls}+\mathrm{hfss}+\mathrm{rsns}+\mathrm{rlns}$

- mlotst (ocean, monthly mean, longitude latitude time)

- so (ocean, monthly mean, longitude latitude olevel time)

- $\quad$ sos (ocean, monthly mean, longitude latitude time)

- tauu (atmos, monthly mean, longitude latitude time)

- tauv (atmos, monthly mean, longitude latitude time)

- to (ocean, monthly mean, longitude latitude olevel time)

- tos (ocean, monthly mean, longitude latitude time)

- uo (ocean, monthly mean, longitude latitude olevel time)

- vo (ocean, monthly mean, longitude latitude olevel time)

- $\quad$ wfpe (atmos, monthly mean, longitude latitude time) $=\mathrm{pr}+$ evspsbl

\section{Observations and Reformat Scripts}

Note: (1) obs4mips data can be used directly without any preprocessing; (2) see headers of reformat scripts for non-obs4mips data for download instructions.

- Dong et al. (2008): Southern Ocean mixed-layer depth from Argo float profiles.

Reformat script: reformat_scripts/obs/reformat_obs_Dong08.ncl.

- ERA-Interim, global atmospheric ECMWF reanalysis: SST, wind stress, precipitation, evaporation, etc.

Reformat scripts

o variables tos, tauu, tauv: reformat_scripts/obs/reformat_obs_ERA-Interim.ncl

o variables $p r$, evspsbl, $h f l s, h f s s, r s n s$, rlns: reformat_scripts/obs/reformat_obs_ERA-Interimsurffluxes.ncl 
- World Ocean Atlas 2009: sea water temperature and salinity

Reformat script: reformat_scipts/obs/reformat_obs_WOA09.ncl.

\section{References}

Antonov, J. I., D. Seidov, T. P. Boyer, R. A. Locarnini, A. V. Mishonov, H. E. Garcia, O. K. Baranova, M. M. Zweng, and D. R. Johnson (2010). World Ocean Atlas 2009, Volume 2: Salinity. S. Levitus, Ed. NOAA Atlas NESDIS 69, U.S. Government Printing Office, Washington, D.C., 184 pp (available at ftp://ftp.nodc.noaa.gov/pub/WOA09/DOC/woa09_vol2 text.pdf).

CDFtools: http://servforge.legi.grenoble-inp.fr/projects/CDFTOOLS

Dong, S., J. Sprintall, S. T. Gille, and L. Talley (2008). Southern Ocean mixed-layer depth from Argo float profiles, J. Geophys. Res., 113, C06013, doi: 10.1029/2006JC004051.

ERA-Interim: http://www.ecmwf.int/en/research/climate-reanalysis/era-interim

ESMF regridding with ncl: http://www.ncl.ucar.edu/Applications/ESMF.shtml

Locarnini, R. A., A. V. Mishonov, J. I. Antonov, T. P. Boyer, H. E. Garcia, O. K. Baranova, M. M. Zweng, and D. R. Johnson (2010). World Ocean Atlas 2009, Volume 1: Temperature. S. Levitus, Ed. NOAA Atlas NESDIS 68, U.S. Government Printing Office, Washington, D.C., 184 pp. (available at ftp://ftp.nodc.noaa.gov/pub/WOA09/DOC/woa09 vol1 text.pdf).

nco: http://nco.sourceforge.net

World Ocean Atlas 2009: https://www.nodc.noaa.gov/OC5/WOA09/pr_woa09.html

\section{Example Plots}
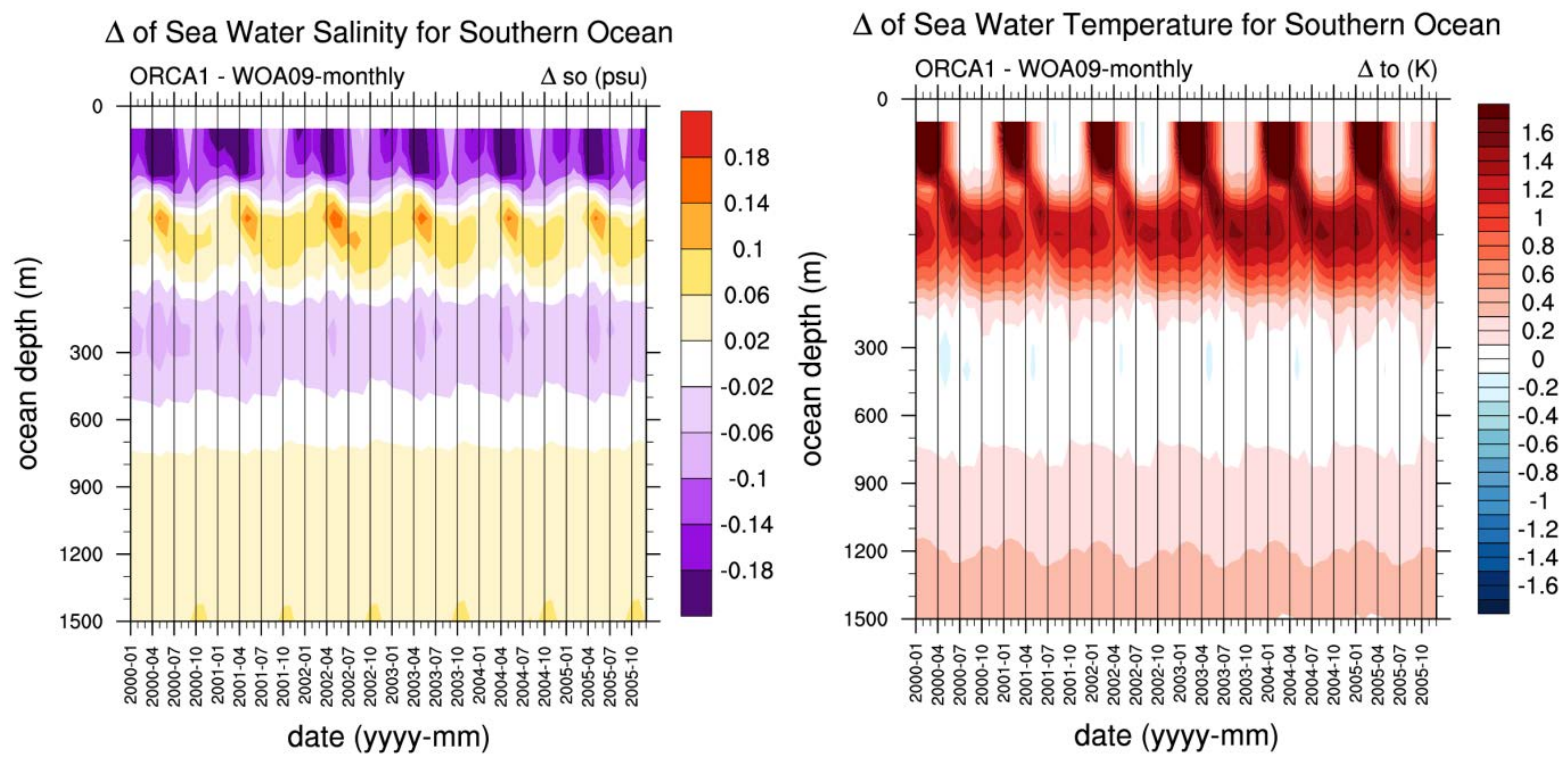
ORCA1 - ERA-Interim
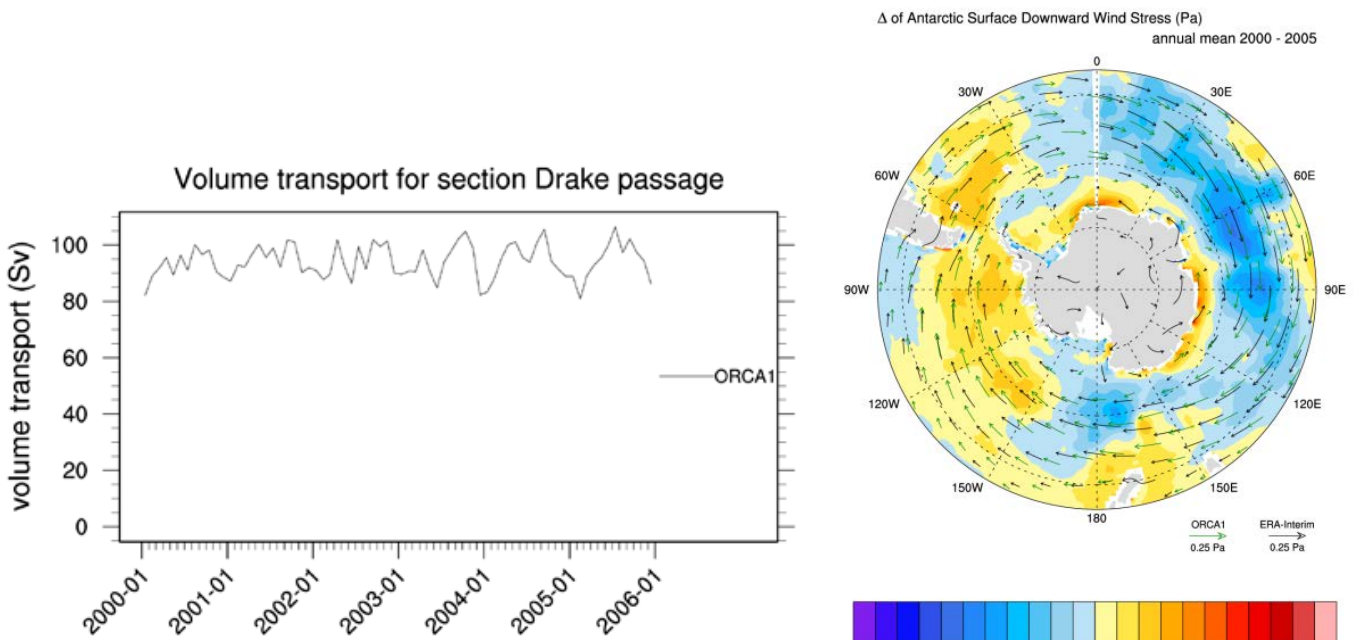

date (yyyy-mm)
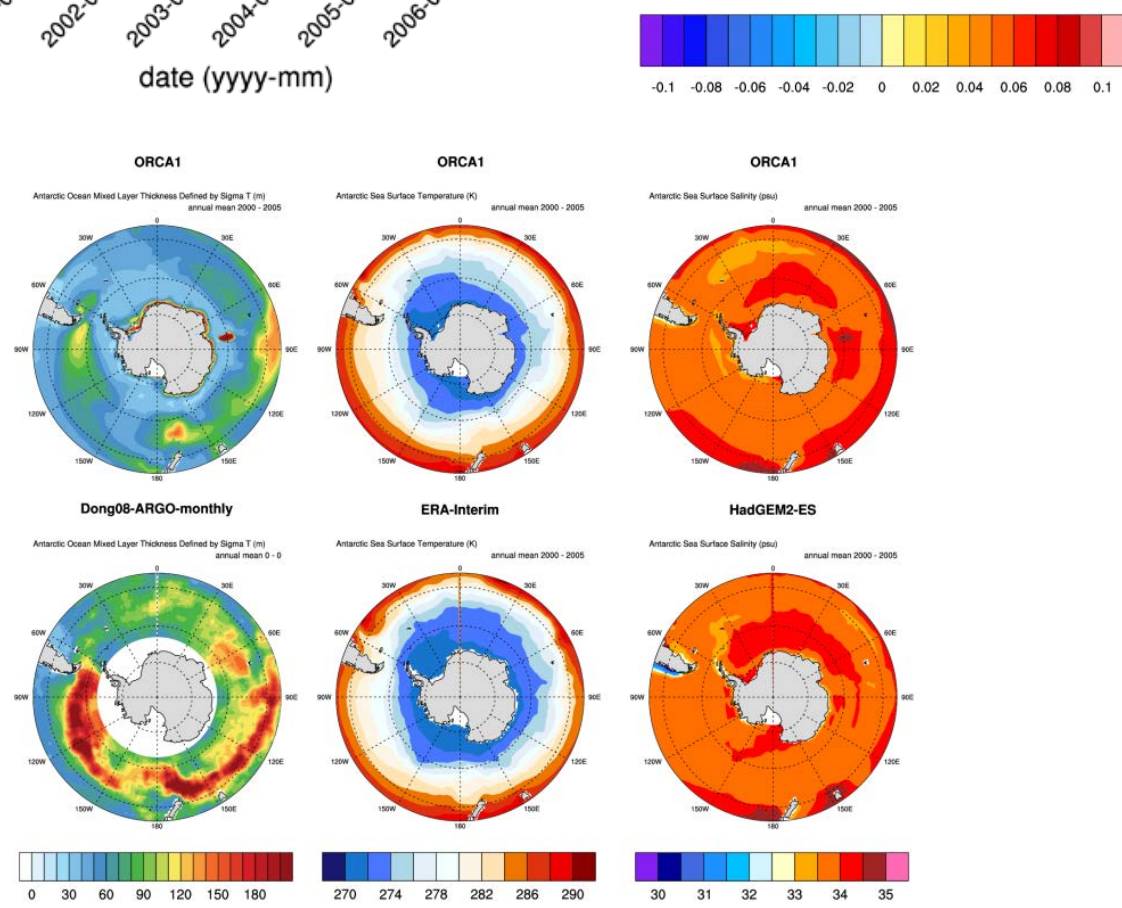


\section{Standardized Precipitation Index (SPI)}

\section{Overview}

For each month, the precipitation over the preceding TIMESCALE months, $\mathrm{x}$, is summed. Then a twoparameter Gamma distribution of cumulative probability, Gamma ${ }_{\alpha, \beta}$, is fitted to the strictly positive TIMESCALE month sums, such that the probability of a non-zero precipitation sum being below a certain value $\mathrm{x}$ corresponds to $\mathrm{Gamma}_{\alpha, \beta}(\mathrm{x})$. We estimate shape parameter $\alpha$ and scale parameter $\beta$ with a maximum likelihood approach. If the estimation does not converge, $\alpha$ and $\beta$ are approximated using empirical relations (Bordi et al., 2001). Accounting for TIMESCALE month periods of no precipitation, occurring at a frequency q, the total cumulative probability distribution of a precipitation sum below $\mathrm{x}, \mathrm{H}(\mathrm{x})$, becomes $\mathrm{H}(\mathrm{x})$ $=\mathrm{q}+(1-\mathrm{q}) * \mathrm{Gamma}_{\alpha, \beta}(\mathrm{x})$. In the last step, a precipitation sum $\mathrm{x}$ is assigned to its corresponding Standardized Precipitation Index (SPI) value by computing the quantile $\mathrm{q}_{\mathrm{N}(0,1)}$ of the standard normal distribution at probability $\mathrm{H}(\mathrm{x})$. The SPI of a precipitation sum $\mathrm{x}$, thus, corresponds to the quantile of the standard normal distribution which is assigned by preserving the probability of the original precipitation sum, $\mathrm{H}(\mathrm{x})$.

\section{Available Namelists and Diagnostics}

Namelists are stored in $\mathrm{nml} /$

- namelist_SPI.xml

Diagnostics are stored in diag_scripts/

- SPI.r

\section{User settings}

User setting files (cfg files) are stored in $\mathrm{nml} / \mathrm{cfg} \_$SPI/

(1) SPR.r

- begin.ref.year: first year of the reference period

- end.ref.year: last year of the reference period

- timescale: valid values are 3,6 and 12 months

• seasons: "ann", "djf", "mam", "jja", "son"

- spi_colorbar_max: color bar range (= -spi_colorbar_max ... + +spi_colorbar_max)

- my.colors: colors for contour plot, e.g., colorRampPalette(c("brown", "orange", "white", "lightblue", "blue"))

- png_width: width of png image

- png_height: height of png image

- png_units: units of png dimensions ("px" = pixels, "in" = inches, "cm" = centimeters, "mm" = millimeters) 
- png_pointsize: the default size of plotted text in points (1/72 inch)

- png_bg: background color, e.g., "white"

\section{Variables}

- $\operatorname{pr}$ (atmos, monthly mean, longitude latitude time)

\section{Observations and Reformat Scripts}

Note: (1) obs4mips data can be used directly without any preprocessing; (2) see headers of reformat scripts for non-obs4mips data for download instructions.

\section{References}

A very good explanation of SPI

Lloyd-Hughes, B. and Saunders, M. A. (2002), A drought climatology for Europe. Int. J. Climatol., 22, 1571-1592. doi: 10.1002/joc.846.

Other standard SPI references

Guttman, N. B. (1999), ACCEPTING THE STANDARDIZED PRECIPITATION INDEX: A CALCULATION ALGORITHM. JAWRA Journal of the American Water Resources Association, 35, 311-322. doi: 10.1111/j.1752-1688.1999.tb03592.x.

McKee, T. B., N. J. Doesken, and J. Kliest, 1993: The relationship of drought frequency and duration to time scales. In Proceedings of the 8th Conference of Applied Climatology, 17-22 January, Anaheim, CA. American Meterological Society, Boston, MA. 179-184.

McKee, T. B, N. J. Doesken, and J. Kliest, 1995: Drought Monitoring with Multiple Time Scales. 9th AMS Conference on Applied Climatology, 15-20 January 1995, Dallas, Texas. 


\section{Example Plots}
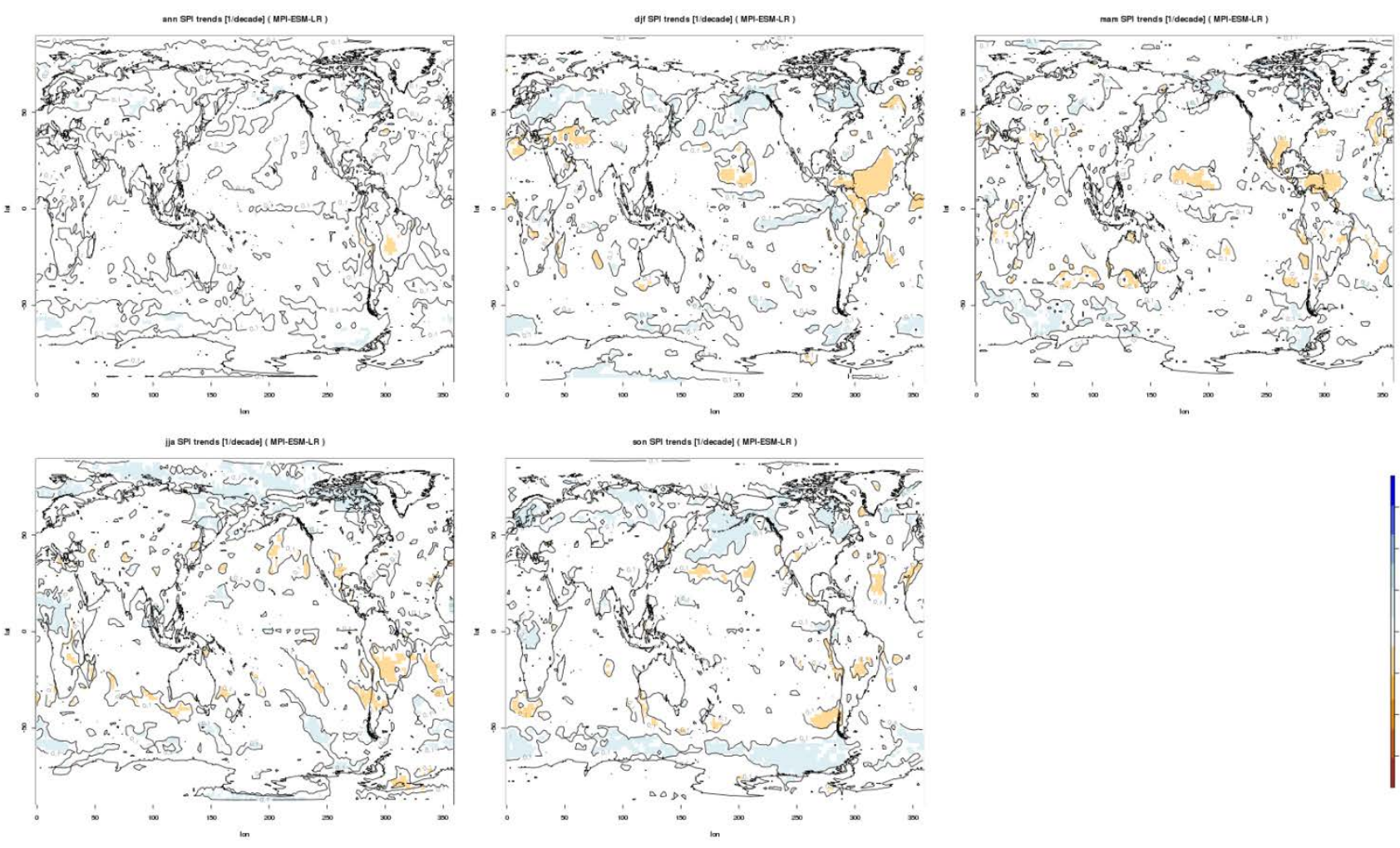


\section{Tropical variability}

\section{Overview}

The available diagnostics are motivated by the work of Li and Xie (2014). In particular, this diagnostics reproduces their Fig. 5 for models and observations/reanalyses, calculating equatorial mean $\left(5^{\circ} \mathrm{N}-5^{\circ} \mathrm{S}\right)$, longitudinal sections of annual mean precipitation (pr), skin temperature (ts), horizontal winds (ua and va) and $925 \mathrm{hPa}$ divergence (derived from the sum of the partial derivatives of the wind components extracted at the $925 \mathrm{hPa}$ pressure level (that is $\mathrm{du} / \mathrm{dx}+\mathrm{dv} / \mathrm{dy}$ ). Latitude cross sections of the model variables are plotted for the equatorial Pacific, Indian and Atlantic Oceans with observational constraints provided by the TRMM3B43-v7 for precipitation, the HadISST for SSTs, and ERA-interim reanalysis for temperature and winds. Latitudinal sections of absolute and normalized annual mean SST and precipitation are also calculated spatially averaged for the three ocean basins. Normalization follows the procedure outlined in Fig. 1 of $\mathrm{Li}$ and Xie (2014) whereby values at each latitude are normalized by the tropical mean $\left(20^{\circ} \mathrm{N}-20^{\circ} \mathrm{S}\right)$ value of the corresponding parameter (e.g., annual mean precipitation at a given location is divided by the $20^{\circ} \mathrm{N}-20^{\circ} \mathrm{S}$ annual mean value). Finally, to assess how models capture observed relationships between SST and precipitation the co-variability of precipitation against SST is calculated for specific regions of the tropical Pacific. This analysis includes calculation of the Mean Square Error (MSE) between model $\mathrm{SST} /$ precipitation and observational equivalents.

\section{Available Namelists and Diagnostics}

Namelists are stored in $\mathrm{nml} /$

- namelist_TropicalVariability.xml

Diagnostics are stored in diag_scripts/

- TropicalVariability.py

- TropicalVariability_EQ.py

- TropicalVariability_wind.py

\section{User settings}

User setting files (cfg files) are stored in $\mathrm{nml} / \mathrm{cfg}$ _Tropical Variability/

(1) TropicalVariability.py, TropicalVariability_EQ.py, TropicalVariability_wind.py

Settings for all diagnostics

- plot_equatorial: switch for equatorial plots (True, False)

- plot_scatter: switch for scatter plots (True, False)

- plot_zonal_means: switch for zonal mean plots (True, False)

- plot_clouds: True, False

- plot_fluxes: True, False 
- mask_unwanted_values: mask values (e.g., missing values) exceeding "mask_limit_low" and "mask_limit_high"(True, False)

- mask_limit_low: lower threshold used for creating a mask (if "mask_unwanted_values" = True)

- mask_limit_high: upper threshold used for creating a mask (if "mask_unwanted_values" = True)

- plot_grid: provides a background grid for relavant plots (True, False)

Settings for equatorial mean plots with precipitation, temperature and winds

- areas: region to process ("Atlantic", "Indian", "Pacific"); each region is defined separately

Definition of regions (“areas”)

- lat_min: min. latitude of region

- lat_max: max. latitude of region

- lon_min: min. longitude of region

- lon_max: max. longitude of region

- prec_min: range of values for precipitation (min.)

- prec_max: range of values for precipitation (max.)

- temp_min: range of values for temperature/SST (min.)

- temp_max: range of values for temperature/SST (max.)

- wind_min: range of values for wind speed (min.)

- $\quad$ wind_max: range of values for wind speed (max.)

- div_min: range of values for divergence (min.)

- div_max: range of values for divergence (max.)

Settings for temperature/precipitation scatter plots

- areas: region to process ("West-Pacific", "Central-Pacific", "East-Pacific")

- seasons: season (annual DJF MAM JJA SON)

- seasonal_limits: if you want to use your own limits (True) or let the code decide (False - values based on observations)

Definition of regions ("areas”) for scatter plots

- lat_min: min. latitude of region

- lat_max: max. latitude of region

- lon_min: min. longitude of region

- lon_max: max. longitude of region

- season_limits_annual: “seasonal” limits (annual means), 4 integer numbers giving 'min. temp.' 'max. temp.' 'min. precip.' 'max. precip.', e.g., 300303410

- season_limits_DJF: same as season_limits_annual, but for Dec-Jan-Feb (DJF)

- season_limits_MAM: same as season_limits_annual, but for Mar-Apr-May (MAM) 
- season_limits_JJA: same as season_limits_annual, but for Jun-Jul-Aug (JJA)

- season_limits_SON: same as season_limits_annual, but for Sep-Oct-Nov (SON)

Definition of the seasons (each season defined separately)

- season_months: numbers of the months covered by the corresponding season, e.g., "12 12 " for the season "DJF"

Settings for zonal means of SST and precipitation

- areas: region to process (Pacific Atlantic Indian)

Definition of the regions ("areas")

- lat_min: min. latitude of region

- lat_max: max. latitude of region

- lon_min: min. longitude of region

- lon_max: max. longitude of region

\section{Variables}

- ts: skin temperature (atmos, monthly mean, time latitude longitude)

- pr: precipitation (atmos, monthly mean, time latitude longitude)

- ua: u-wind (atmos, monthly mean, time plevel latitude longitude)

- va: v-wind (atmos, monthly mean, time plevel latitude longitude)

\section{Observations and Reformat Scripts}

Note: (1) obs4mips data can be used directly without any preprocessing; (2) see headers of reformat scripts for non-obs4mips data for download instructions.

- HadISST: skin Temperature (ts) / sea surface temperature (SST)

Reformat script: reformat_scripts/obs/reformat_obs_HadISST.ncl

- $\quad$ TRMM-L3 (pr, monthly means - obs4mips)

- $\quad$ ERA-Interim (u-wind, v-wind)

Reformat script: reformat_scripts/obs/reformat_obs_ERA-Interim.ncl

\section{References}

Li, G., and , S.-P. Xie (2014), Tropical Biases in CMIP5 Multimodel Ensemble: The Excessive Equatorial Pacific Cold Tongue and Double ITCZ Problems. J. Climate, 27, 1765-1780. doi: http://dx.doi.org/10.1175/JCLI-D-13-00337.1. 


\section{Example Plots}
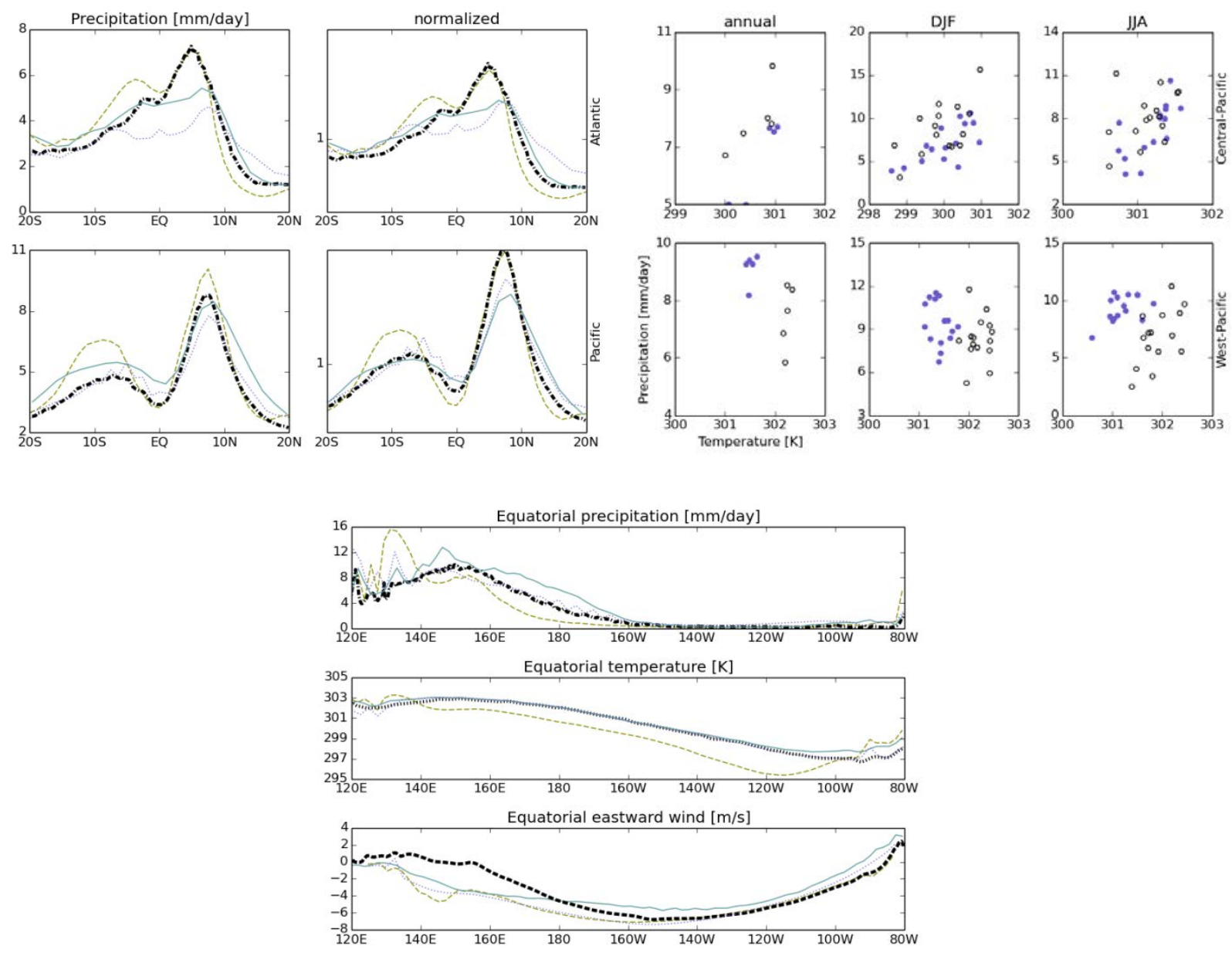


\section{West African Monsoon}

\section{Overview}

West Africa is a critical region for climate models (e.g., Cook and Vizy, 2006; Roehrig et al., 2013). Roehrig et al. (2013) show that although state-of-the-art CMIP5 models can capture many features of the West African monsoon, they have not yet reached a sufficient degree of maturity that makes them trustable to anticipate climate changes and their impacts in this region, especially with regard to rainfall. Therefore, along the process of climate model development and evaluation, it is crucial for model developers and users to have at their disposal a set of synthetic and simple diagnostics that provide them an overall vision of the representation of the West African monsoon in their model or set of models. Such diagnostics are implemented in this namelist.

\section{Available Namelists and Diagnostics}

Namelists are stored in $\mathrm{nml} /$

- namelist_WAMonsoon.xml

- namelist_WAMonsoon_daily.xml

Diagnostics are stored in diag_scripts/

- WAMonsoon_10W10E_1D_basic.ncl: same as WAMonsoon_10W10E_3D_basic.ncl but for a 2D variable (e.g., precipitation, potential temperature at $850 \mathrm{hPa}$ ).

- WAMonsoon_10W10E_3D_basic.ncl: computes the zonal average over $10^{\circ} \mathrm{W}-10^{\circ} \mathrm{E}$ of a 3 dimensional variable (e.g., zonal wind, meridional wind, potential temperature). It is then averaged over the JJAS season and plotted as a latitude-level transect over West Africa.

- WAMonsoon_autocorr.ncl: similar to WAMonsoon_isv_filtered.ncl, except that it computes the 1day autocorrelation of intraseasonal anomalies of any field (e.g., precipitation), as a measure of convection persistence.

- WAMonsoon_contour_basic.ncl: computes the average of any 2-dimensional field over the JJAS season and plots it as a latitude-longitude map zoomed over West Africa. It is used for precipitation, 2-m air temperature and 850-hPa potential temperature (heat low signature).

- WAMonsoon_precip_IAV.ncl: plots the interannual variability of precipitation averaged over JJAS and over the Sahel $\left(10^{\circ} \mathrm{N}-20^{\circ} \mathrm{N}, 10^{\circ} \mathrm{W}-10^{\circ} \mathrm{E}\right)$.

- WAMonsoon_isv_filtered.ncl: filters any 2-dimensional field, computes the standard deviation of the filtered field over the JJAS season and plots it on a latitude-longitude map zoomed over West Africa. High-pass and band-pass filter based on the Lanczos filtering method are available. Basically, this is used for computing the 90-day high pass or 3-10-day bandpass filtered precipitation or outgoing longwave radiation standard deviation, namely the intraseasonal and synoptic (African easterly waves) standard deviation. Data are first interpolated on a common grid before any computations. We advise a $1^{\circ} \times 1^{\circ}$ grid for precipitation (reference is GPCP version 1.2, 1DD, available on this grid) and a $2.5^{\circ} \times 2.5^{\circ}$ grid for OLR (reference is from NOAA satellites, and available on this grid). 
- WAMonsoon_precip_seasonal.ncl: computes the mean monthly annual cycle of any 2-dimensional variable averaged over a given latitude-longitude box and plots it for the given models and reference data set. It is used for precipitation and 2-m air temperature averaged over the Sahel $\left(10^{\circ} \mathrm{N}-20^{\circ} \mathrm{N}\right.$, $\left.10^{\circ} \mathrm{W}-10^{\circ} \mathrm{E}\right)$.

- WAMonsoon_wind_basic.ncl: computes the average of zonal and meridional wind component over the JJAS season and plots it as a latitude-longitude map for a given level (200 and $700 \mathrm{hPa}$ ). Zonal wind is in shading and total wind is in vector. The map is zoomed over West Africa.

\section{User settings}

User setting files (cfg files) are stored in nml/cfg_WAMonsoon/

(1) WAMonsoon_10W10E_1D_basic.ncl

\section{Required diag_script_info attributes}

- latrange: min. and max. latitude for plot (2-elemen array)

- lonrange: min. and max. longitude for plot (2-element array)

- minmax: lower and upper limit of values in plot (2-element array)

- season: season, typically "JJAS"

Optional diag_script attributes

- cn_levels_mean_basic: contour levels for mean plot (n-element array)

- cn_levels_mean_diff_basic: contour levels for difference plot (n-element array)

- cn_levels_stddev_basic: contour levels for standard deviation plot (n-element array)

- cn_levels_stddev_diff_basic: contour levels for difference stdandard deviation plot (n-element array)

- legendPos: position of legend ("TopRight”, "BottomRight”, “TopLeft”, "BottomLeft”)

- multi_model_mean: calculate multi-model mean (“y", "n")

- plottype_lbres: plot labelbar (True, False)

- $\quad$ styleset: stylesheet to use ("CMIP5", "DEFAULT")

- x_gridlines: display gridline along $x$-axis (“"y", " $n$ ")

- y_gridlines: display gridline along y-axis (“y", " $n$ ")

(2) WAMonsoon_10W10E_3D_basic.ncl

\section{Required diag_script_info attributes}

- cn_levels_mean_basic: contour levels for mean plot (n-element array)

- cn_levels_mean_diff_basic: contour levels for difference plot (n-element array)

- latrange: min. and max. latitude for plot (2-element array)

- lonrange: min. and max. longitude for plot (2-element array) 
- levrange: max. and min. pressure (Pa) for plot (2-element array)

- plottype_lbres: handle for labelbar settings (True, False)

- season: season, typically "JJAS"

- plot_stddev: switch for plotting stdandard deviation (True, False)

- diff_colormap: filename and full path for colormap for difference plot, e.g., "diag_scripts/aux/WAMonsoon/cmap_difference_theta.rgb"

\section{Optional diag_script_info attributes}

- cn_levels_mean_basic: contour levels for mean plot (n-element array)

- cn_levels_mean_diff_basic: contour levels for difference plot (n-element array)

(3) WAMonsoon_autocorr.ncl

Required diag_script_info attributes

- cn_levels_corr_basic: contour levels for correlation plot (n-element array)

- cn_levels_corr_diff_basic: contour levels for correlation difference plot (n-element array)

- latrange: min. and max. latitude for plot (2-elemen array)

- lonrange: min. and max. longitude for plot (2-element array)

- sahel_latrange: min. and max. latitude of "Sahel" region (2-element array)

- sahel_lonrange: min. and max. longitude of "Sahel" region (2-element array)

- season: season, typically "JJAS"

- destgrid: destination grid for ESMF regridding ("1x1", "2.5x2.5")

- styleset: stylesheet to use ("CMIP5", “DEFAULT”)

- my_region: label for Monsoon region, e.g., "WA"

- filter_hp: value for high pass filter

- filter_type: "hp" = high pass filter, "bp" = band pass filter

- filter_weights: filter weights

- filter_min, filter_max: required for band pass filter only

(4) WAMonsoon_contour_basic.ncl

\section{Required diag_script_info attributes}

- cn_levels_mean_basic: contour levels for mean plot (n-element array)

- cn_levels_mean_diff_basic: contour levels for difference plot (n-element array)

- latrange: min. and max. latitude for plot (2-element array)

- lonrange: min. and max. longitude for plot (2-element array)

- season: season, typically "JJAS"

- my_region: label for Monsoon region, e.g., "WA" 
- plot_stddev: switch for plotting stdandard deviation (True, False)

- cn_levels_stddev_basic: contour levels for standard deviation plot (only required if plot_stddev $=$ True) (n-element array)

- cn_levels_stddev_diff_basic: contour levels for standard deviation difference plot (only required if plot_stddev $=$ True) (n-element array)

- diff_colormap: filename and full pathname of colormap for difference plots, e.g., "diag_scripts/aux/WAMonsoon/cmap_difference.rgb"

(5) WAMonsoon_precip_IAV.ncl, WAMonsoon_precip_seasonal.ncl

Required diag_script_info attributes

- cn_levels_mean_basic: contour levels for mean plot (n-element array)

- cn_levels_mean_diff_basic: contour levels for difference plot (n-element array)

- cn_levels_stddev_basic: contour levels for standard deviation plot (n-element array)

- cn_levels_stddev_diff_basic: contour levels for difference stdandard deviation plot (n-element array)

- diff_colormap: filename and full pathname of colormap for difference plots, e.g., "diag_scripts/aux/WAMonsoon/cmap_difference.rgb"

- latrange_seasonal: min. and max. latitude of crop region for plot (2-element array)

- lonrange_seasonal: min. and max. longitude of crop region for plot (2-element array)

- $\quad$ season: season, typically "JJAS"

- styleset: stylesheet to use ("CMIP5", "DEFAULT")

- multi_model_mean: calculate multi-model mean ("“y", "n")

- my_region: label for Monsoon region, e.g., "WA"

- $\quad$ supporting_gridlines: display supporting grid line ("y", " $\mathrm{n}$ ")

(6) WAMonsoon_isv_filtered.ncl

\section{Required diag_script_info attributes}

- $\quad$ season: season, typically "JJAS"

- destgrid: destination grid for ESMF regridding ("1x1", "2.5x2.5")

- styleset: stylesheet to use ("CMIP5", "DEFAULT")

- latrange_basic: min. and max. latitude for plot (2-elemen array)

- lonrange_basic: min. and max. longitude for plot (2-element array)

- diff_colormap: filename and full pathname of colormap for difference plots, e.g., "diag_scripts/aux/WAMonsoon/cmap_difference.rgb"

- cn_levels_stddev_basic: contour levels for standard deviation plot (n-element array)

- cn_levels_stddev_diff_basic: contour levels for difference standard deviation plot (n-element array) 
- plot_norm: plot normalized stdandard deviation (True, False)

- cn_levels_stddev_norm_basic: contour levels for normalized standard deviation plot (n-element array)

- cn_levels_stddev_norm_diff_basic: contour levels for normalized standard deviation difference plot (n-element array)

- sahel_latrange: min. and max. latitude of "Sahel" region (2-element array)

- sahel_lonrange: min. and max. longitude of "Sahel” region (2-element array)

- filter_type: "hp" = high pass filter, "bp" = band pass filter

- filter_hp: value for high pass filter

- filter_weights: filter weights

- filter_min, filter_max: required for band pass filter only

- my_region: label for Monsoon region, e.g., "WA"

(7) WAMonsoon_wind_basic.ncl

Required diag_script_info attributes

- $\quad$ styleset: stylesheet to use ("CMIP5", "DEFAULT")

- season: season, typically "JJAS"

- latrange_basic: min. and max. latitude for plot (2-element array)

- lonrange_basic: min. and max. longitude for plot (2-element array)

- cn_levels_mean_basic: contour levels for mean plot (n-element array)

- cn_levels_mean_diff_basic: contour levels for difference plot (n-element array)

- diff_colormap: filename and full pathname of colormap for difference plots, e.g., "diag_scripts/aux/WAMonsoon/cmap_difference_wind.rgb"

- cn_levels_stddev_basic: contour levels for standard deviation plot (n-element array) (if plot_stddev $=$ True)

- cn_levels_stddev_diff_basic: contour levels for difference standard deviation plot (n-element array) (if plot_stddev $=$ True)

- plottype_lbres: plot labelbar (True, False)

- my_region: label for Monsoon region, e.g., "WA"

- use_for_contour: switch for kind of contour ("speed", "zonal” (ua), "meridional” (va))

- plot_stddev: plot standard deviation (True, False)

\section{Variables}

- $\operatorname{pr}$ (atmos, monthly mean, longitude latitude time)

- tas (atmos, monthly mean, longitude latitude time)

- rlut (atmos, monthly mean, longitude latitude time) 
- $\operatorname{rsut}$ (atmos, monthly mean, longitude latitude time)

- rlutcs (atmos, monthly mean, longitude latitude time)

- $\quad$ rsutcs (atmos, monthly mean, longitude latitude time)

- rlds (atmos, monthly mean, longitude latitude time)

- rsds (atmos, monthly mean, longitude latitude time)

- ua (atmos, monthly mean, longitude latitude plev time)

- va (atmos, monthly mean, longitude latitude plev time)

- ta (atmos, monthly mean, longitude latitude plev time)

- $\operatorname{pr}$ (atmos, daily mean, longitude latitude time)

- rlut (atmos, daily mean, longitude latitude time)

\section{Observations and Reformat Scripts}

Note: (1) obs4mips data can be used directly without any preprocessing; (2) see headers of reformat scripts for non-obs4mips data for download instructions.

- ERA-Interim Reanalysis (tas, ua, va)

Reformat script: reformat_scripts/obs/reformat_obs_ERA-Interim.ncl

- GPCP monthly (pr) - obs4mips

- CERES-EBAF (TOA and derived surface radiation fluxes) - obs4mips

- GPCP Version 1.2 , daily and $1^{\circ} \times 1^{\circ}(\mathrm{pr})$ - obs4mips

- Daily NOAA OLR

Reformat script: reformat_scripts/obs/reformat_obs_NOAA-PSD-Interp-rlut.ncl

\section{References}

Cook, K. H. and E. K. Vizy, 2006: Coupled model simulations of the West African monsoon system: Twentieth- and twenty-first-century simulations. J. Climate, 19, 3681-3703.

Roehrig, R., D. Bouniol, F. Guichard, F. Hourdin, and J.-L. Redelsperger, 2013: The Present and Future of the West African Monsoon: A Process-Oriented Assessment of CMIP5 Simulations along the AMMA Transect. J. Climate, 26, 6471-6505. doi: http://dx.doi.org/10.1175/JCLI-D-12-00505.1. 


\section{Example Plots}
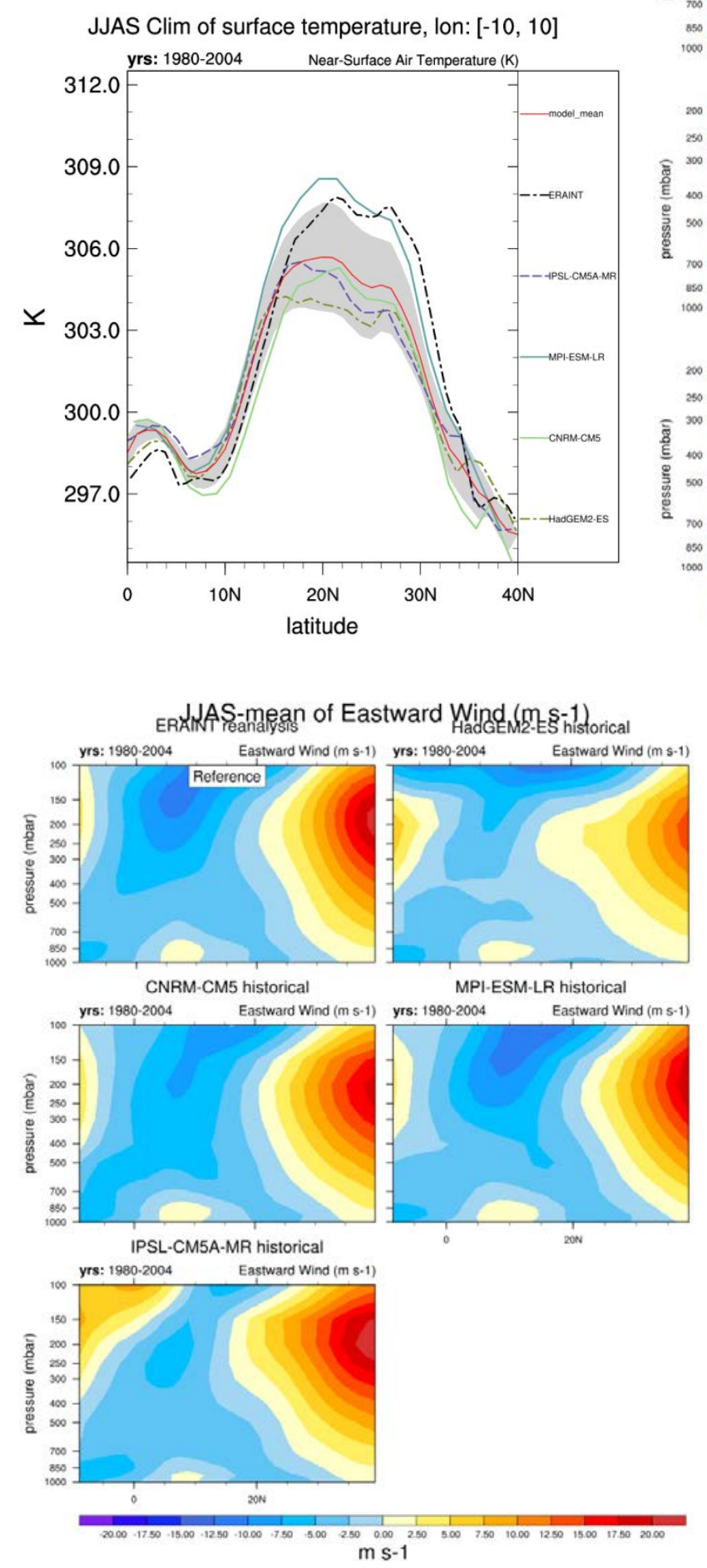

ERAlNAST-meanan of Potential Temperature,

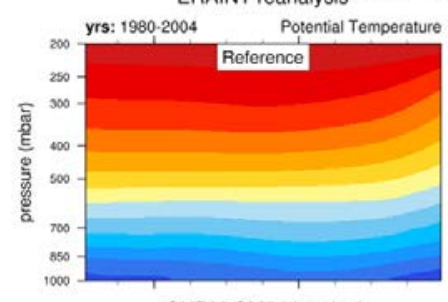

$$
\text { CNRM-CM5 historical }
$$

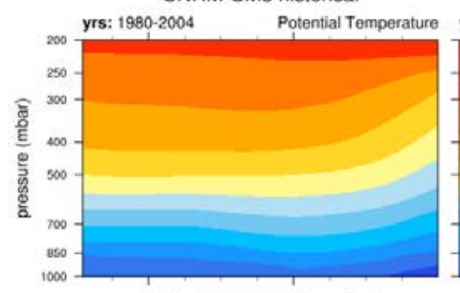

IPSL-CM5A-MR historical

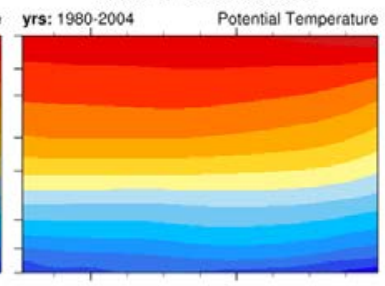

MPI-ESM-LR historical

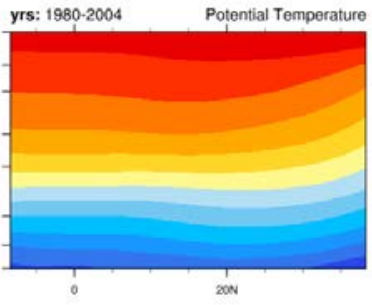

row

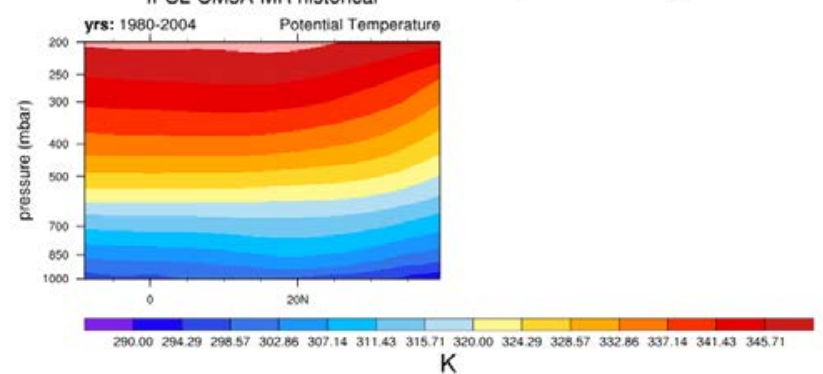

$\mathrm{K}$

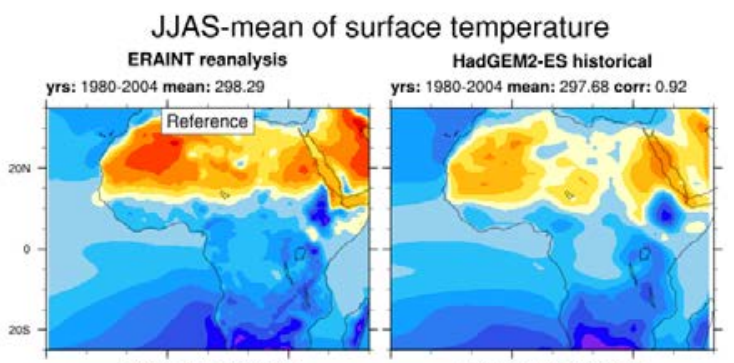

CNRM-CM5 historical

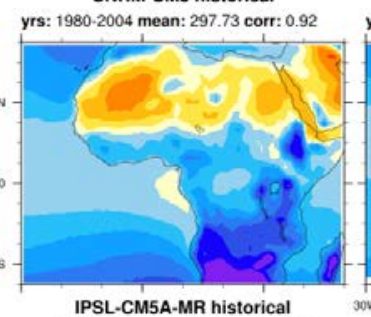

MPI-ESM-LR historical yrs: 1980-2004 mean: 298.82 corr: 0.93
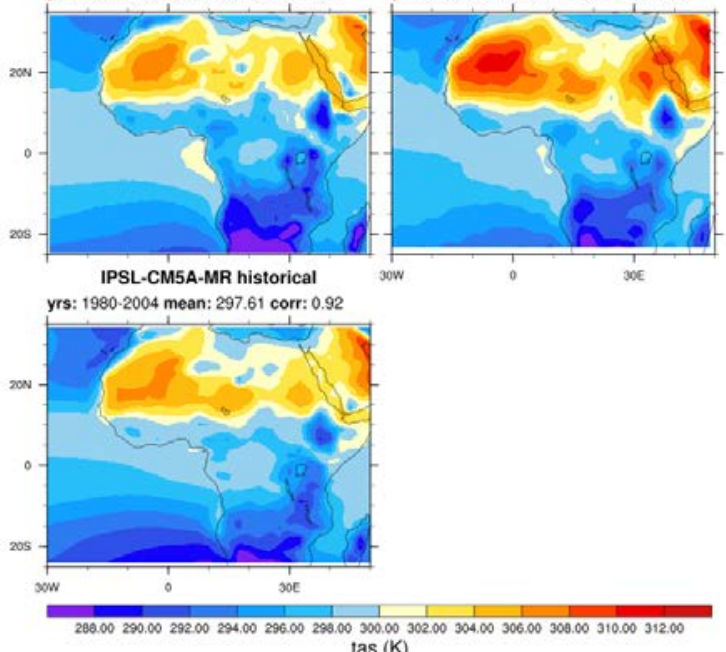
JJAS-corr 1-day autocorr of 1-90-day Precipitation Rate
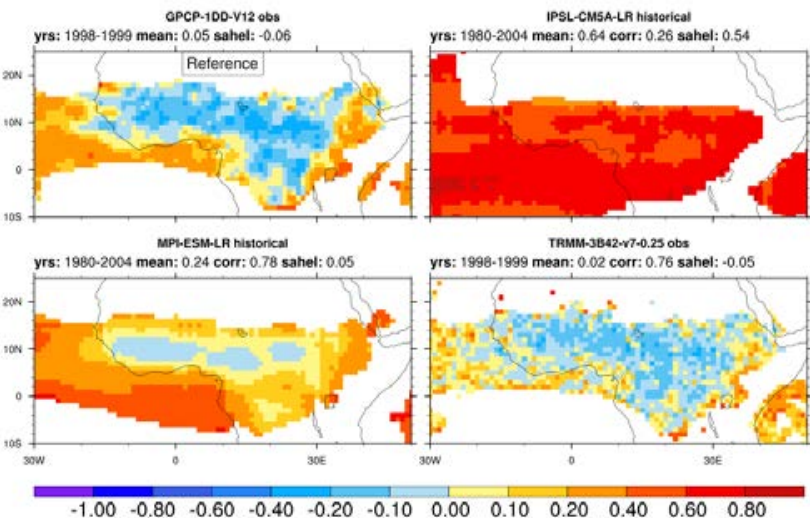

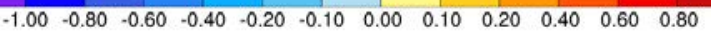

JJAS-stddev stddev of 3-10-day TOA Outgoing Longwave Radiation

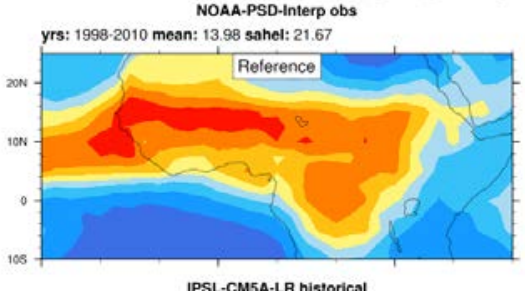

yrs: 1980.2004 mean: 10.15 sahel: 13.19

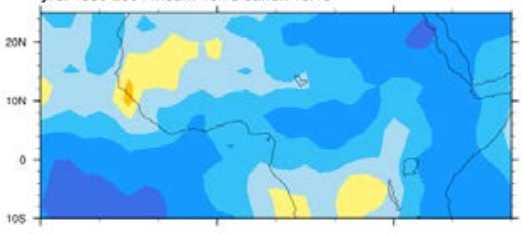

MPI-ESM-LR historical

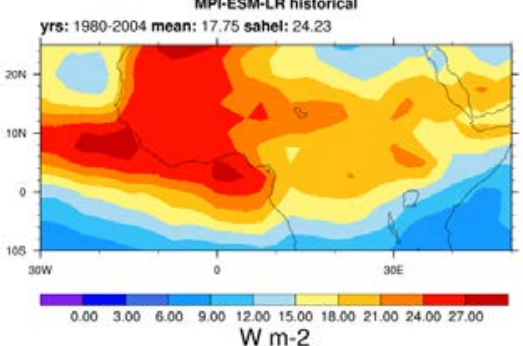

JJAS Clim of Longwave cloud radiative effect, lon: $[-10,10]$

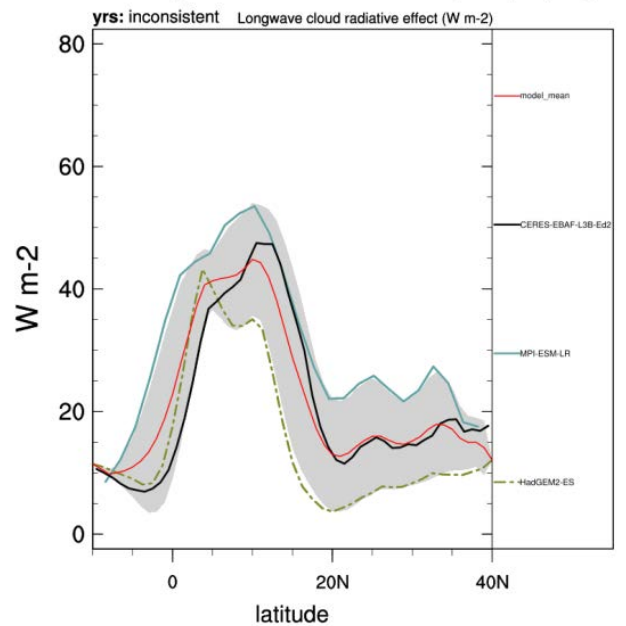

Seasonal cycle

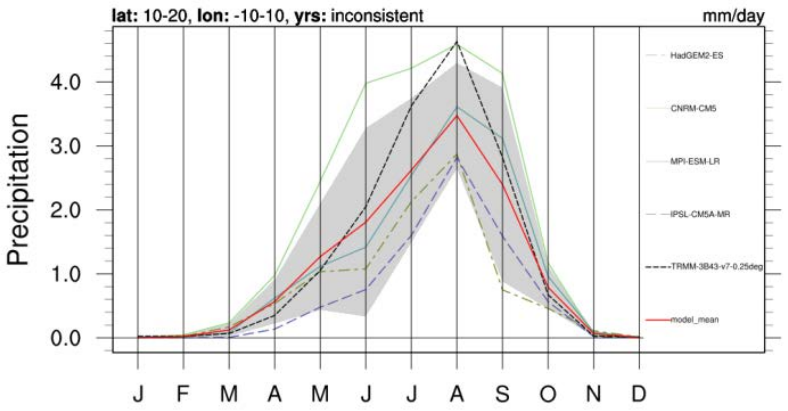

JJAS-mean of $700 \mathrm{hPa}$ Zonal

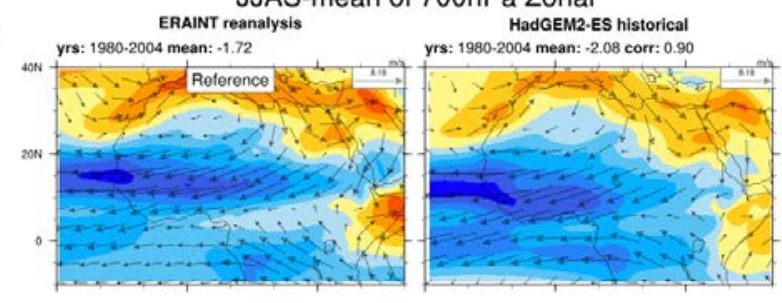

CNRM-CMS historical

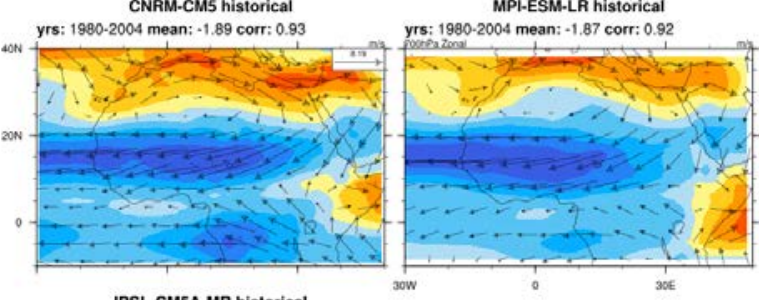

IPSL-CM5A-MR historical

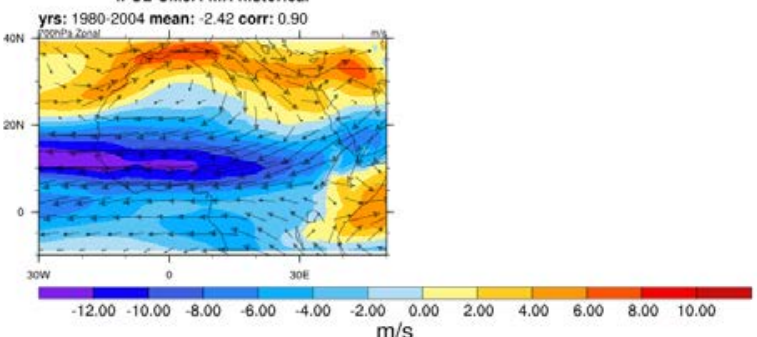

JJAS Clim of Surface Downwelling Shortwave Radiation, Ion: [-10, 10]

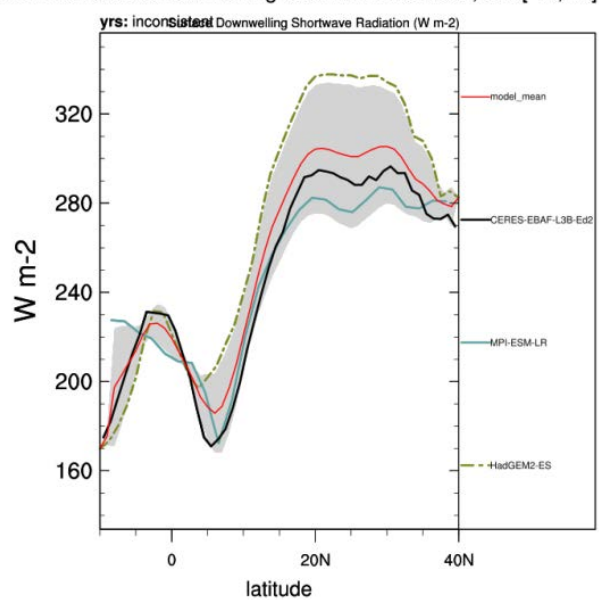

